\title{
Dynamics of the NGC 4636 globular cluster system
}

\section{Improved constraints from a large sample of globular cluster velocities $\star, \star \star$}

\author{
Y. Schuberth ${ }^{1,2}$, T. Richtler ${ }^{2}$, M. Hilker ${ }^{3}$, R. Salinas ${ }^{2,4}$, B. Dirsch ${ }^{5}$, and S. S. Larsen ${ }^{6}$ \\ 1 Argelander-Institut für Astronomie, Universität Bonn Auf dem Hügel 71, 53121 Bonn, Germany \\ 2 Universidad de Concepción, Departamento de Astronomía, Casilla 160-C, Concepción, Chile \\ e-mail: tom@astroudec.cl \\ 3 European Southern Observatory, Karl-Schwarzschild-Str. 2, 85748 Garching, Germany \\ 4 Finnish Centre for Astronomy with ESO (FINCA), University of Turku, Väisäläntie 20, 21500 Piikkiö, Finland \\ 5 Friedrich-Ebert-Gymnasium Bonn, Ollenhauerstr. 5, 53113 Bonn, Germany \\ ${ }^{6}$ Department of Astrophysics, IMAPP, Radboud University Nijmegen, PO Box 9010, 6500 GL Nijmegen, The Netherlands
}

Received 25 May 2010 / Accepted 26 April 2012

\begin{abstract}
We present new radial velocities for 289 globular clusters around NGC 4636, the southernmost giant elliptical galaxy of the Virgo cluster. The data were obtained with FORS2/MXU at the Very Large Telescope. Together with data analysed in an earlier study, we now have a sample of 460 globular cluster velocities out to a radius of $12 \operatorname{arcmin}(60 \mathrm{kpc})$ available - one of the largest of its kind. This new data set also provides a much more complete angular coverage. Moreover, we present new kinematical data of the inner stellar population of NGC 4636. We perform an updated Jeans analysis, using both stellar and GC data, to better constrain the dark halo properties. We find a stellar M/L-ratio of 5.8 in the $R$-band, higher than expected from single stellar population synthesis. We model the dark halo by cored and cuspy analytical halo profiles and consider different anisotropies for the tracer populations. Properties of NFW halos lie well within the expected range of cosmological simulations. Cored halos give central dark matter densities, which are typical for elliptical galaxies of NGC 4636's luminosity. The surface densities of the dark matter halos are higher than those of spiral galaxies. We compare the predictions of modified Newtonian dynamics with the derived halo properties and find satisfactory agreement. NGC 4636 therefore falls onto the baryonic Tully-Fisher relation for spiral galaxies. The comparison with the X-ray mass profile of Johnson et al. (2009, ApJ, 706, 980) reveals satisfactory agreement only if the abundance gradient of hot plasma has been taken into account. This might indicate a general bias towards higher masses for X-ray based mass profiles in all systems, including galaxy clusters, with strong abundance gradients.
\end{abstract}

Key words. galaxies: elliptical and lenticular, cD - galaxies: kinematics and dynamics - galaxies: individual: NGC 4636 galaxies: star clusters: general

\section{Introduction}

NGC 4636 is a remarkable elliptical galaxy. Situated at the Southern border of the Virgo galaxy cluster, and thus not in a very dense environment, its globular cluster system (GCS) exhibits a richness, which one does not find in other galaxies of comparable luminosity in a similar environments (Kissler et al. 1994; Dirsch et al. 2005). Therefore, NGC 4636 offers the opportunity to employ globular clusters (GCs) as dynamical tracers to investigate its dark halo out to large radii, which is rarely given. In our first study (Schuberth et al. 2006, hereafter Paper I), we measured 174 GC radial velocities to confirm the existence of a dark halo, previously indicated by X-ray analyses (e.g. Loewenstein \& Mushotzky 2003) and tried to constrain its mass. Paper I also gives a summary of the numerous works related to NGC 4636 until 2006, which we do not want to repeat here. Dirsch et al. (2005) presented a wide-field photometry in the Washington system of the globular cluster system

\footnotetext{
* Based on observations made with ESO Telescopes at the Paranal Observatories under programme ID 075.B-0762.

$\star \star$ Appendices are available in electronic form at http://www . aanda.org
}

of NGC 4636, which was the photometric base for Paper I as well as for the present work. Also there, the interested reader will find a summary of earlier works. Noteworthy peculiarities of NGC 4636 include the appearance of the supernova 1937A (a bona fide Ia event, indicative of presence of an intermediateage population), the high FIR-emission (Temi et al. 2003, 2007), the chaotic X-ray features in the inner region (Jones et al. 2002; Baldi et al. 2009), pointing to feed-back effects from supernovae or enhanced nuclear activity in the past. Table 1 summarises the basic parameters related to NGC 4636.

Since NGC 4636 is X-ray bright, it has been the target of numerous X-ray studies (for earlier work see Matsushita et al. 1998; Jones et al. 2002; Loewenstein \& Mushotzky 2003, and references therein), offering the possibility to compare X-ray based mass profiles with stellar dynamical mass profiles, using a data base, which is not found elsewhere for a non-central elliptical galaxy.

The most recent X-ray based analysis of the mass profile is from Johnson et al. (2009) whose work is based on Chandra $\mathrm{X}$-ray data. The new feature in their work is the inclusion of an abundance gradient in the X-ray gas. We will show that, if this gradient is accounted for, the resulting $\mathrm{X}$-ray mass profile is 
Table 1. NGC 4636 basic data.

\begin{tabular}{|c|c|c|}
\hline \multicolumn{3}{|c|}{ NGC 4636 basic data } \\
\hline Other names & UGC 07878, VCC 1939 & NED \\
\hline Position (2000) & $12^{\mathrm{h}} .42^{\mathrm{m}} .49^{\mathrm{s}} .8+02^{\circ} .41^{\prime} 16^{\prime \prime}$ & NED \\
\hline & 190.707792 & \\
\hline Galactic coordinates & $l=297.75^{\circ}$ & \\
\hline Galactic extinction & $A_{B}=0.050$ & (1) \\
\hline & $A_{B}=0.118$ & (2) \\
\hline Distance & $D=17.5 \mathrm{Mpc}$ & (Sect. 1) \\
\hline Scale & \multicolumn{2}{|l|}{$1^{\prime \prime}=85 \mathrm{pc} \quad 1^{\prime}=5.09 \mathrm{kpc}$} \\
\hline Distance modulus & \multicolumn{2}{|l|}{$(m-M)=31.22$} \\
\hline Heliocentric velocity & $v_{\text {helio }}=906 \pm 7 \mathrm{~km} \mathrm{~s}^{-1}$ & (3) \\
\hline Hubble type & $\mathrm{E} 0+$ & $\mathrm{R} 3 \mathrm{C}$ \\
\hline Ellipticity & $\epsilon=0.15$ & (4) \\
\hline Position angle & $\mathrm{PA}=150^{\circ}$ & $\mathrm{R} 3 \mathrm{C}$ \\
\hline Effective radius & $R_{\mathrm{e}}=101^{\prime \prime} .7(=8.64 \mathrm{kpc})$ & (4) \\
\hline Age & $13.5 \pm 3.6 \mathrm{Gyr}$ & (5) \\
\hline Metallicity & $\begin{array}{c}Z=0.023 \pm 0.006 \\
{[\alpha / \mathrm{Fe}]=0.29 \pm 0.06}\end{array}$ & (5) \\
\hline$\frac{\alpha / \text { Fe }}{\text { Total blue mag }}$ & \multirow{2}{*}{$\begin{array}{c}b_{\mathrm{T}}=9.78 \\
B_{\mathrm{T}}=-21.05\end{array}$} & $\frac{(J)}{(6)}$ \\
\hline Absolute blue mag & & \\
\hline Colours & $\begin{array}{l}U-B=0.50 \\
B-V=0.87 \\
V-R=0.67 \\
V-I=1.30\end{array}$ & (6) \\
\hline $\begin{array}{l}\text { Stellar pop } M / L \\
\text { Dynamical } M / L\end{array}$ & $M / L_{I}=3.74$ & $\begin{array}{l}(7) \\
(8)\end{array}$ \\
\hline X-ray luminosity & $L_{\mathrm{X}}=1.78 \pm 0.10 \times 10^{41} \mathrm{ergs} / \mathrm{s}$ & (9) \\
\hline Nuclear X-ray emission & $\leq 2.7 \times 10^{38} \mathrm{ergs} / \mathrm{s}$ & (10) \\
\hline Central black hole & $M_{\mathrm{SMBH}} \sim 8 \times 10^{7} M_{\odot}$ & $(11)$ \\
\hline
\end{tabular}

Notes. UGC = Uppsala General Catalogue of Galaxies (Nilson 1973), VCC = Virgo Cluster Catalogue (Binggeli et al. 1985), NED = NASA/IPAC Extragalactic Database (http://nedwww. ipac . caltech.edu), R3C = Third Reference Catalogue of Bright Galaxies (de Vaucouleurs et al. 1991).

References. (1) Burstein \& Heiles (1982); (2) Schlegel et al. (1998); (3) Paper I; (4) Bender et al. (1994); (5) Annibali et al. (2007); (6) Prugniel \& Heraudeau (1998); (7) Maraston et al. (2003); (8) Kronawitter et al. (2000); (9) Forman et al. (1985); (10) Loewenstein et al. (2001); (11) Merritt \& Ferrarese (2001).

in good agreement with the one derived from our GC analysis, which may indicate the need to respect abundance gradients, if present, in any X-ray analysis.

Two recent publications on the NGC 4636 GCs are from Park et al. (2010) and Lee et al. (2010), who present about 100 new Subaru spectra of NGC 4636 GCs and discuss their kinematics in combination with the data from Schuberth et al. (2006). For the sake of brevity, we postpone a detailed comparison of the different samples to a later publication.

Our data from Paper I have also been used by Chakrabarty $\&$ Raychaudhury (2008) who employed their own code to obtain a mass profile.

In the analysis presented in Paper I, the most important source of uncertainty is the sparse data at large radii: the dispersion value derived for the outermost radial bin changes drastically depending on whether two GCs with extreme velocities are discarded or not. If these data are included, the estimate of the mass enclosed within $30 \mathrm{kpc}$ goes up by a factor of $\sim 1.4$ and the inferred dark halo has an extremely large ( $\gtrsim 100 \mathrm{kpc})$ scale radius.

Moreover, the data presented in Paper I have a very patchy angular coverage since the observed fields were predominantly placed along the photometric major axis of NGC 4636 (see Fig. 4, right panel). To achieve a more complete angular coverage and to better constrain the enclosed mass and the shape of the NGC 4636 dark halo, we obtained more VLT FORS 2 MXU data, using the same instrumental setup as in our previous study.

In our present study, an important difference to Paper I is the distance, which is of paramount importance in the dynamical discussion. In Paper I, we adopted a distance of $15 \mathrm{Mpc}$, based on surface brightness fluctuation (SBF) measurements (Tonry et al. 2001), but already remarked that we consider this value to be a lower limit. A more recent re-calibration of the SBF brings the galaxy even closer to $13.6 \mathrm{Mpc}$ (Jensen et al. 2003).

On the other hand, the method of globular luminosity functions revealed a distance of 17.5 Mpc (Kissler et al. 1994; Dirsch et al. 2005). We do not claim a superiority of GCLFs over SBFs, but one cannot ignore the odd findings which result from adopting the short distance. The specific frequency of GCs would assume a value rivalling that of central cluster galaxies (Dirsch et al. 2005). Moreover, the stellar M/L for which in Paper I we already adopted a very high value of 6.8 in the $R$-band, would climb to 7.5 for a distance of $13.6 \mathrm{Mpc}$, which almost doubles the value expected for an old metal-rich population (e.g. Cappellari et al. 2006). This is under the assumption that the total mass in the central region, for which we present independent kinematical data, is dominated by the stellar mass (the "maximal disk" assumption). The alternative, namely that NGC 4636 is dark matter dominated even in its centre, would be intriguing, but would make NGC 4636 a unique case. To adopt a larger distance is the cheapest solution. The explanation for the discrepancy between SBF and GCLF distance might lie in some effect enhancing the fluctuation signal, either by an intermediateage population (which in turn would require a lower M/L) or another small-scale structure, which went so far undetected. In the following, we adopt a distance of $17.5 \mathrm{Mpc}$. Thus, $1^{\prime}$ is $\sim 5.1 \mathrm{kpc}$ and $1^{\prime \prime}=85 \mathrm{pc}$.

This paper is organised as follows: in Sect. 2, we give a brief description of the spectroscopic observations and the data reduction. In Sect. 3, we present the combined data set, before discussing the spatial distribution and the photometric properties of the GC sample in Sect. 4. In Sect. 5, we define the subsamples used for the dynamical study. The line-of-sight velocity distributions of the different subsamples are presented in Sect. 6. In Sect. 7, the subsamples are tested for rotation, and in Sect. 8, the velocity dispersion profiles are shown. In Sect. 9 we present new stellar kinematics for NGC 4636 and in Sect. 10 we summarise the theoretical framework for the Jeans modelling. The mass profiles are shown in Sect. 11. Sections 12 and 13 give the discussion and the conclusions, respectively.

\section{Observations and data reduction}

The data were acquired using the same instrumental setup as described in Paper I. Therefore, we just give a brief description here.

\subsection{VLT observations}

The observations were carried out in service mode at the European Southern Observatory (ESO) Very Large Telescope 


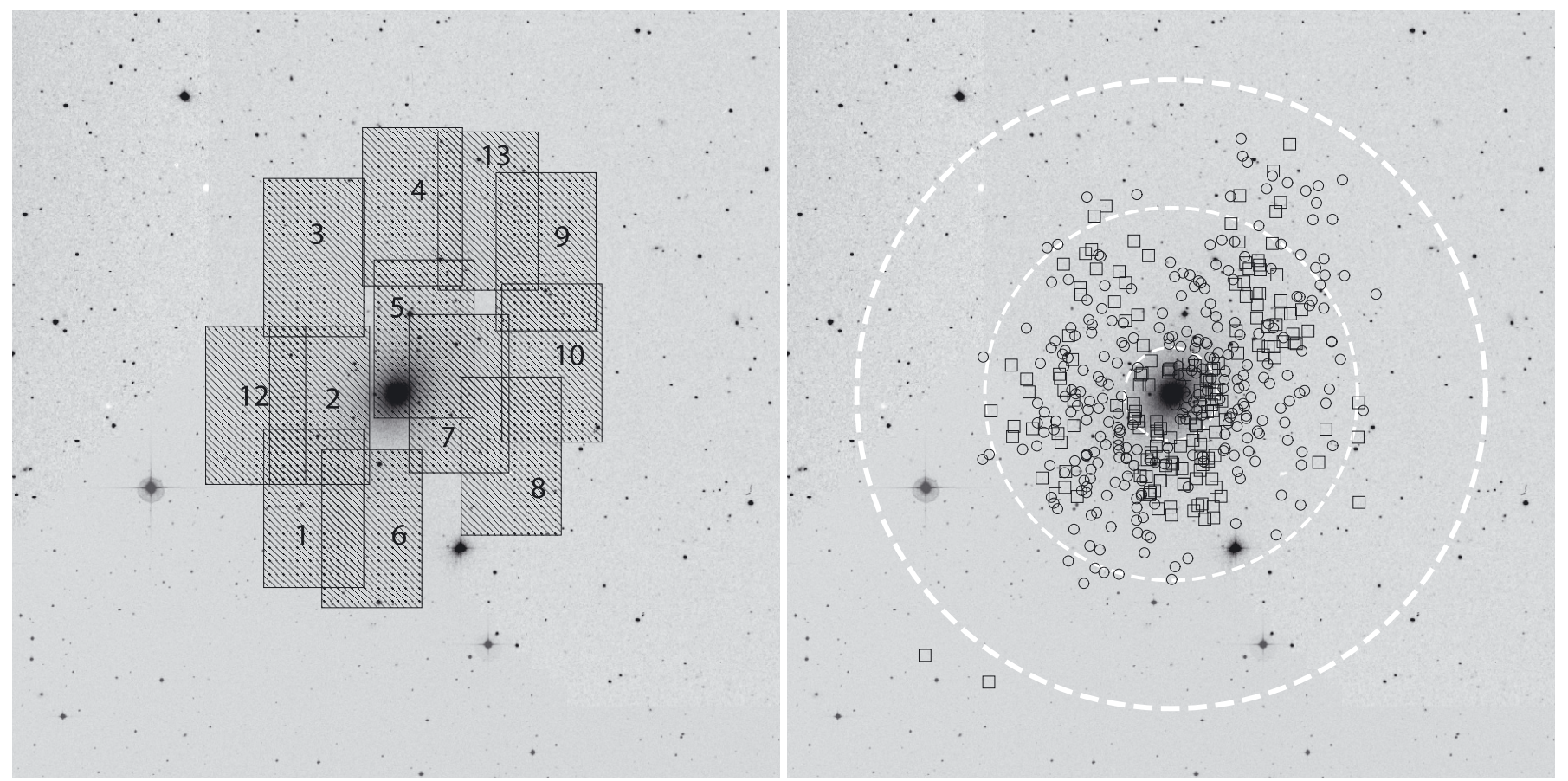

Fig. 1. NGC 4636 GC spectroscopic data. The $33^{\prime} \times 33^{\prime}$ DSS image shown in both panels is centred on NGC 4636, North is to the top and East is to the left. Left: location of the new fields. Note that only the inner $4.3 \times 6.8$ of the FORS 2 field-of-view are shown, since the positions of the slits are confined to this area. Right: all NGC 4636 velocity-confirmed GCs. Squares show the data set from Paper I, circles the new data. The white dashed circles have radii of $2^{\prime} .0,8.0$ and 13.5 , corresponding to approximately 10,41 , and $69 \mathrm{kpc}$ (at $D=17.5 \mathrm{Mpc}$ ), respectively.

(VLT) facility on Cerro Paranal, Chile. We used the FORS 2 (FOcal Reducer/low dispersion Spectrograph) equipped with the Mask EXchance Unit (MXU). The programme ID is $075 . \mathrm{B}-0762(\mathrm{~B})$.

Pre-imaging of twelve fields, shown in the left panel of Fig. 1, was obtained in April 2005. The GC candidates were selected from the photometric catalogue by Dirsch et al. (2005). The targets have colours in the range $0.9<C-R<2.1$ and $R$-band magnitudes brighter than $22.5 \mathrm{mag}$.

The spectroscopic masks were designed using the ESO FORS Instrumental Mask Simulator (FIMS) software. In contrast to Paper I, where object and sky spectra were obtained from different slits, we chose to observe the GC candidates through longer slits and to extract the background from the same slit. The slits for point-sources have a width of $1^{\prime \prime}$ and a length of $4 "$. The spectroscopic observations were carried out in service mode in the period May 2nd to June 7th, 2005. We used the Grism 600 B which gives a resolution of $\sim 3 \AA$. A total of twelve masks were observed with exposure times between 3600 and 5400 s. To minimise the contamination by cosmic-ray hits, the observation of each mask was divided into two or three exposures. A summary of the MXU observations is given in Table 2 .

\subsection{Data reduction}

Prior to bias subtraction, the fsmosaic-script, which is part of the FIMS-software was used to merge the two CCD-exposures of all science and calibration frames. The spectra were traced and extracted using the IRAF apall package.

For the extraction of point-sources, we chose an aperture size of 3 (binned) pixels, corresponding to $0.75^{\prime \prime}$. In each slit, we interactively defined an emission-free background region, the pixel values of which were averaged and subtracted off the spectrum during the extraction. This sky-subtraction was found to work very well, only the strongest atmospheric emission lines left residuals. The spectra were traced using the interactive mode of apall, and the curve fit to the trace was a Chebyshev polynomial of order $3-11$. The use of this wide range of polynomials is motivated by the fact that the characteristics of the bottom CCD ("slave") deviate from those of top ("master") CCD. Besides having a worse point spread function, the traces of spectra on the "slave" CCD are more contorted. Thus, the tracing of spectra on the "slave" CCD required the use of the higher-order polynomials. On the "master" frame, an order of 3-5 proved to be sufficient.

The wavelength calibration is based on $\mathrm{Hg}-\mathrm{Cd}-\mathrm{He}$ arclamp exposures obtained as part of the standard calibration plan. The one-dimensional arc spectra were calibrated using the IRAF task identi fy. Typically, 17-22 lines were identified per spectrum and the dispersion solution was approximated by a 5th-order Chebyshev polynomial. The rms-residuals of these fits were about $0.05 \AA$.

The wavelength calibrations were obtained during day-time, after re-inserting the masks and with the telescope pointed at zenith. Consequently, the offsets introduced by instrument flexure and more importantly, the finite re-positioning accuracy of MXU have to be compensated for. To derive the corresponding velocity corrections we proceeded as follows: the wavelength of the strong [OI] $5577 \AA$ atmospheric emission line was measured in all raw spectra of a given mask as a function of the slit's location on the CCD (orthogonal to the dispersion axis). The correction for each aperture was determined from a 2nd order polynomial fit to the wavelength-position data. The average magnitude of this correction was $\sim 30 \mathrm{~km} \mathrm{~s}^{-1}$. On a given mask, the wavelength drift between the lower edge of the slave chip to the top edge of the master chip corresponded to velocity differences between 10 and $90 \mathrm{~km} \mathrm{~s}^{-1}$. 
Table 2. Summary of NGC 4636 VLT FORS2/MXU observations.

\begin{tabular}{|c|c|c|c|c|c|c|c|}
\hline \multirow{2}{*}{$\begin{array}{l}\text { Mask ID } \\
\text { F01 }\end{array}$} & \multicolumn{2}{|c|}{$\begin{array}{c}\text { Centre position } \\
(\mathrm{J} 2000)\end{array}$} & \multirow{2}{*}{$\begin{array}{l}\text { Obs. date } \\
\text { 2005-05-04 }\end{array}$} & \multirow{2}{*}{$\begin{array}{c}\text { Seeing } \\
0.73\end{array}$} & \multirow{2}{*}{$\begin{array}{c}\text { \# Exp. } \\
3\end{array}$} & \multirow{2}{*}{$\begin{array}{c}\text { Exp. time } \\
\text { (s) }\end{array}$} & \multirow{2}{*}{$\begin{array}{r}\text { \# Slits } \\
57\end{array}$} \\
\hline & $12: 43: 04.0$ & $+02: 36: 20.0$ & & & & & \\
\hline F 02 & $12: 43: 03.0$ & $+02: 40: 46.0$ & 2005-05-05 & 0.90 & 3 & 5400 & 67 \\
\hline F 03 & $12: 43: 04.0$ & $+02: 47: 07.0$ & 2005-05-02 & $1 . " 00$ & 3 & 5400 & 65 \\
\hline F 04 & $12: 42: 47.0$ & $+02: 49: 18.0$ & 2005-05-07 & 0.90 & 2 & 3600 & 64 \\
\hline F 05 & $12: 42: 45.0$ & $+02: 43: 37.0$ & 2005-05-05 & 0.71 & 3 & 5400 & 63 \\
\hline F06 & $12: 42: 54.0$ & $+02: 35: 28.0$ & 2005-05-31 & $0 ! 71$ & 2 & 3600 & 71 \\
\hline F 07 & $12: 42: 39.0$ & $+02: 41: 16.0$ & 2005-05-31 & $0 ! 90$ & 2 & 3600 & 75 \\
\hline F 08 & $12: 42: 30.0$ & $+02: 38: 35.0$ & 2005-05-05 & 0.66 & 2 & 3600 & 67 \\
\hline F 09 & $12: 42: 24.0$ & $+02: 47: 22.0$ & 2005-05-05 & 0.63 & 3 & 5100 & 63 \\
\hline F 10 & $12: 42: 23.0$ & $+02: 42: 35.2$ & 2005-06-01 & 0.58 & 3 & 5400 & 68 \\
\hline F 12 & $12: 43: 14.0$ & $+02: 40: 46.0$ & 2005-06-02 & 0.50 & 3 & 5400 & 62 \\
\hline F 13 & $12: 42: 34.0$ & $+02: 49: 07.0$ & 2005-06-07 & $0 ! 76$ & 3 & 5400 & 68 \\
\hline
\end{tabular}

Notes. ESO program ID 75.B-0762(B). The seeing values were measured from the acquisition images obtained prior to the corresponding spectroscopic observations.
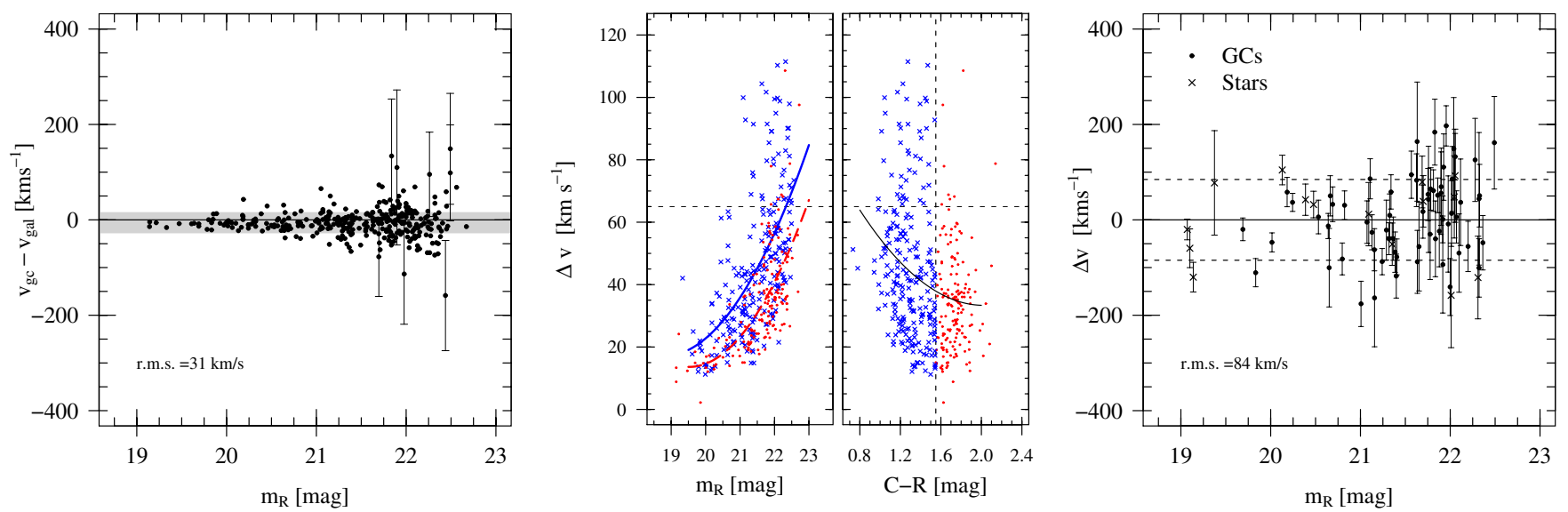

Fig. 2. Velocity uncertainties. Left: comparison of the GC velocity estimates obtained with the two different template spectra. For graphic convenience, the error-bars (the two fxcor-uncertainties added in quadrature) are only shown for objects where the estimates differ by more than $75 \mathrm{~km} \mathrm{~s}^{-1}$. The grey area shows the region encompassing 68 per cent of the data points. Middle: velocity uncertainties as determined by fxcor. Left sub-panel: fxcor-uncertainty vs. $R$-magnitude. Crosses and dots represent blue and red GCs, respectively. The solid and dashed curve show a quadratic fit to the blue and red GC data, respectively. Right sub-panel: fxcor-uncertainty vs. colour. The dashed line at $C-R=1.55$ indicates the colour dividing blue from red GCs. The solid line illustrating the increase of the uncertainties towards bluer colours is a $2 \mathrm{nd}$ order polynomial fit to the data. The dashed lines at $\Delta v=65 \mathrm{~km} \mathrm{~s}^{-1}$ show the limit adopted for the sample definition in Sect. 5.2. Right: comparison of the duplicate velocity measurements. Dots and crosses represent GCs and stars, respectively. The dashed lines show the rms scatter of $84 \mathrm{~km} \mathrm{~s}^{-1}$ found for the GCs.

\subsection{Velocity determination}

To determine the radial velocities of our spectroscopic targets, we proceeded as described in Paper I. We used the same spectrum of the dwarf elliptical galaxy NGC 1396 $\left(v=815 \pm 8 \mathrm{~km} \mathrm{~s}^{-1}\right.$, Dirsch et al. 2004) as template, and the velocities were measured using the IRAF fxcor task, which is based upon the technique described in Tonry \& Davis (1979).

To double check our results, we also used the spectrum of one of the brightest globular clusters found in this new dataset (object f12-24, $v_{\text {helio }}=980 \pm 15 \mathrm{~km} \mathrm{~s}^{-1}, m_{R}=19.9 \mathrm{mag}, C-R=$ 1.62) as a template. Note that these are the same templates we used in our recent study of the NGC 1399 GCS Schuberth et al. (2010).

The wavelength range used for the correlation was $\lambda \lambda 4100-5180 \AA$. The blue limit was chosen to be well within the domain of the wavelength calibration. Towards the red, we avoid the residuals from the relatively weak telluric nitrogen emission line at $5199 \AA$. In the few spectra affected by cosmic ray residuals or bad pixels, the wavelength range was adjusted interactively.

The left panel of Fig. 2 shows the difference of the velocities determined using the the "GC" and the "galaxy" template as function of $R$-magnitude. As expected, the scatter increases for fainter magnitudes. The rms of the residuals is $35 \mathrm{~km} \mathrm{~s}^{-1}$, which is comparable to the mean velocity uncertainties returned by $\mathrm{fxcor}\left(\overline{\Delta v}_{\mathrm{gc}}=37, \overline{\Delta v}_{\mathrm{gal}}=48 \mathrm{~km} \mathrm{~s}^{-1}\right)$. For each spectrum, we adopt the velocity with the smaller fxcor-uncertainty.

The middle panel of Fig. 2 shows the fxcor-uncertainties as function of $R$-magnitude (left sub-panel). The solid (dashed) curve is a quadratic fit to the blue (red) GCs. While the uncertainties increase for fainter objects, one also notes that, at a given magnitude, the blue GCs, on average, have larger velocity 

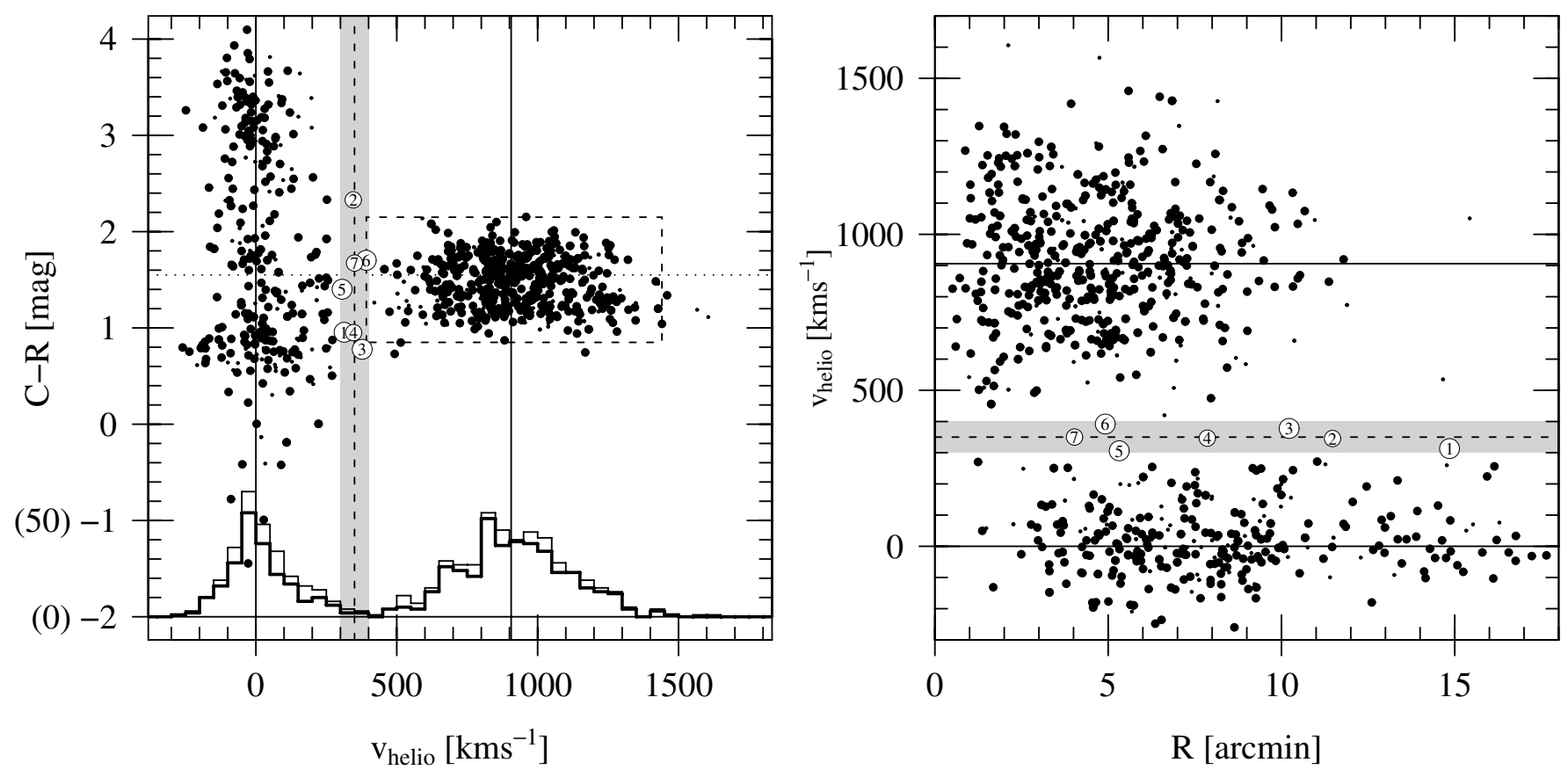

Fig. 3. Velocity distributions: separating GCs from foreground stars. In both panels, the dashed line at $350 \mathrm{~km} \mathrm{~s}^{-1}$ shows the velocity cut used in Paper I, and the grey region has a width of $100 \mathrm{~km} \mathrm{~s}^{-1}$. In both panels, objects in this region are shown as white dots and numbered in decreasing order of the radial distance. The solid line indicates the systemic velocity of NGC $4636\left(v=906 \mathrm{~km} \mathrm{~s}^{-1}\right)$. Left: colour vs. heliocentric velocity. The dashed box shows the range of parameters for the GCs analysed in Paper I (cf. Table 3 therein). Large symbols show objects with velocity uncertainties $\Delta v \leq 65 \mathrm{~km} \mathrm{~s}^{-1}$. The histograms ( $y$-axis labels in parenthesis) have a bin width of $50 \mathrm{~km} \mathrm{~s}^{-1}$, and the thick line shows the objects with $\Delta v \leq 65 \mathrm{~km} \mathrm{~s}^{-1}$. Right: heliocentric velocity vs. projected galactocentric distance. Again, large symbols show objects with $\Delta v \leq 65 \mathrm{~km} \mathrm{~s}{ }^{-1}$.

uncertainties. The same trend can be seen in the right sub-panel, where we plot colour-dependence of the uncertainties: the bluer the objects, the larger the uncertainties. This trend is likely due to the paucity of spectral features in the spectra of bluest, i.e. most metal-poor GCs.

\section{The combined data set}

In this section, we describe the database used for the dynamical analysis. The final catalogue as given in Table C. 1 combines the new velocities with the data presented in Paper I and the photometry by Dirsch et al. (2005, D+05 hereafter).

Our data base has 893 entries, (547 new spectra, 346 velocities from the catalogues presented in Paper I). The new spectra come from 463 unique objects $(327$ GCs and 136 Galactic foreground stars ${ }^{1}$ ). Of these, 289 GCs and 116 stars were not targeted in the previous study. The spectra of background galaxies were discarded from the catalogue at the stage of the fxcor velocity determination, and there were no ambiguous cases.

Thus, including the 171 (170) unique GCs (foreground stars), from Paper I, the data set used in this work comprises the velocities of 460 individual bona-fide NGC 4636 GCs and 286 foreground stars.

\subsection{Photometry database}

As in Paper I, we used the Washington photometry by $\mathrm{D}+05$ to assign $C-R$ colours and $R$-band magnitudes to the spectroscopic targets.

\footnotetext{
1 Following Paper I, we consider objects with $v_{\text {helio }}$ below $350 \mathrm{~km} \mathrm{~s}^{-1}$ as foreground stars (see Sect. 3.3).
}

For some objects with velocities in the range expected for GCs, however, no photometric counterpart was found in the final photometric catalogue published by $\mathrm{D}+05$. The reason for this is that the D+05 catalogue lists the point sources in the field, which were selected using the DAOPhot II (Stetson 1987, 1992) " $\chi$ " and "sharpness" parameters. While this selection, as desired, rejects extended background galaxies, it also removes those GCs whose images deviate from point sources. The GCs which were culled from the photometric data set fall into two categories, the first encompassing objects whose images have been distorted because of their location near a gap of the CCD mosaic, a detector defect (e.g. a bad row) or a region affected by a saturated star. The second group are GCs whose actual sizes are large enough to lead to slightly extended images on the MOSAIC images which have a seeing of about 1 ." 0 .

Using the "raw" version of the photometry database, we were able to recover colours and magnitudes for 154 objects, 80 of which are GCs (according to the criteria defined below in Sect. 3.3). As can be seen from the left and middle panels of Fig. 5, many of the brightest GCs were excluded from the $\mathrm{D}+05$ catalogue.

\subsection{Duplicate measurements}

In our catalogue, we identify 131 (94) duplicate, 8 (7) triple measurements, and 607 (359) objects were measured only once (the corresponding numbers for the GCs are given in parenthesis). For objects with multiple measurements we combine the measurements using the velocity uncertainties as weights. The velocities from the duplicate measurements are compared in the right panel of Fig. 2. The rms scatter of $84 \mathrm{~km} \mathrm{~s}^{-1}$ found for the GCs is about twice the average velocity uncertainty quoted 

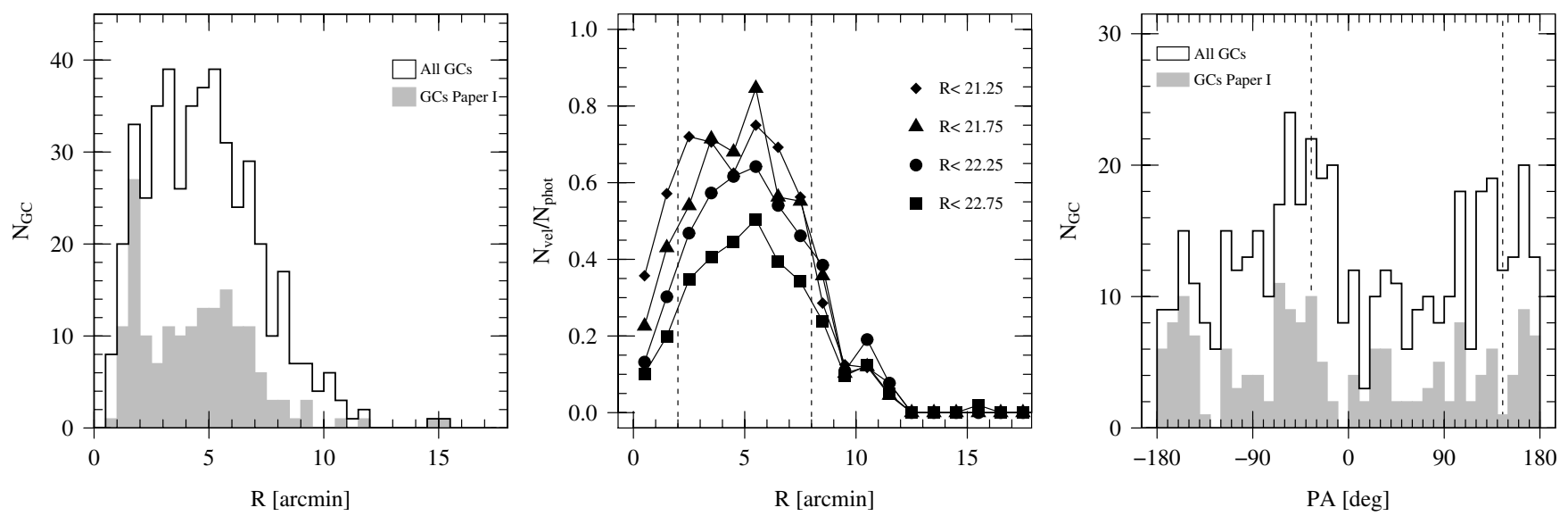

Fig. 4. Spatial distribution of the velocity-confirmed GCs. Left panel: radial distribution of the spectroscopic GC sample. The unfilled and grey histograms show the full sample and the data from Paper I, respectively. Middle panel: radial completeness for different faint-end magnitude limits: for the GCs from the D+05 catalogue, we plot the ratio of GCs with velocity measurements with respect to the total number of GC candidates with colours in the range $(0.73 \leq C-R \leq 2.15)$ found for the kinematic sample (cf. Fig. 5 , left panel). Right panel: azimuthal distribution of the GCs. The position angle (PA) is measured North over East and the dashed lines indicate the location of the photometric major axis of NGC 4636. The histogram styles are the same as in the left panel.

by the cross-correlation programme. Random offsets in velocity might be introduced by targets which are not centred in the slit in the direction of the dispersion direction.

\subsection{Separating GCs from Galactic foreground stars}

In the left panel of Fig. 3, we plot the $C-R$ colour versus heliocentric velocity: the GCS of NGC 4636 occupies a welldefined area in the velocity-colour plane. The foreground stars, clustering around Zero velocity, have a much broader colour distribution ${ }^{2}$.

As in Paper I, we adopt $v_{\text {helio }}=350 \mathrm{~km} \mathrm{~s}^{-1}$ as lower limit for bona fide NGC 4636 GCs. Although both classes of objects are well separated in velocity space, there is a number of uncertain cases: the seven objects with velocities in the range $300-400 \mathrm{~km} \mathrm{~s}^{-1}$ (indicated by the grey areas in both panels of Fig. 3) merit closer scrutiny. These objects are discussed in Sect. 5.1.2 where we remove the likely outliers from our sample.

The following section gives a description of the photometric properties and the spatial distribution of the 460 bona fide NGC 4636 GCs in our velocity catalogue.

\section{Properties of the GC sample}

Our kinematic sample now comprises a total of $460 \mathrm{GCs}$, which is more than 2.5 times the number of GCs used in Paper I. After NGC 1399 (Richtler et al. 2004, 2008; Schuberth et al. 2010) with almost $700 \mathrm{GC}$ velocities, Cen A (Woodley et al. 2010) with about 560 velocities, and M 87 (Strader et al. 2011) with 737 velocities, this is the fourth-largest $\mathrm{GC}$ velocity sample to date.

\subsection{Spatial distribution}

The spatial distribution of the GCs with velocity measurements is shown in Fig. 4. The left panel plots the distribution of the

\footnotetext{
2 The data set includes foreground stars which do not fulfil the colour criteria used for the GC candidate selection because all spectra, including those of the bright stars used for mask alignment, were extracted.
}

galactocentric distances of the GCs as unfilled histogram. The azimuthal distribution is shown in the right panel. Compared to Paper I (grey histogram), we have now achieved a more homogeneous coverage, especially filling the gaps in the radial range $2^{\prime}-5^{\prime}$, in the area near the minor axis of NGC 4636 .

Figure 4 (middle panel) shows our estimate of the radial completeness of the kinematic GC sample: for radial bins of $1^{\prime}$ width, we compute the ratio of GCs with velocity measurements to the number of candidate GCs for the published $\mathrm{D}+05$ catalogue (for consistency, GCs not listed in D+05 are not considered here). Since bright GC candidates were preferred over faint ones for the spectroscopic observations, the completeness level changes significantly depending on the faint-end limiting magnitude, and the corresponding curves are shown with different symbols in the middle panel of Fig. 4. In the innermost bins, the completeness is very low, since the mask positions were chosen to avoid these parts where the light of NGC 4636 would dominate the GC spectra. As can already be seen from Fig. 1 (right panel), the spatial coverage in the area between about $2^{\prime}$ and $7^{\prime}$ is very good, and indeed the completeness peaks around a radial distance of 5'5. Beyond about $8^{\prime}$, the number of GCs with velocity measurements becomes very small, hence the rapidly declining completeness. Apart from the sparse spatial coverage, this is also due to the fact that the total number of GCs expected in the outer regions is small, owing to the steeply declining number density profiles. This is also illustrated by Fig. 3 (middle panel), where, for radii beyond $10^{\prime}$ we find a number of foreground stars but almost no GCs.

\subsection{Luminosity distribution}

The left panel of Fig. 5 compares the luminosity distribution of the spectroscopic sample to the luminosity function (LF) of the GCs in the photometric catalogue. For a direct comparison between the two data sets, we only consider objects in the radial range $2^{\prime}<R<8^{\prime}$, i.e. where the spatial completeness of the spectroscopic sample is largest. For GCs brighter than $m_{R} \simeq$ 21.4, the luminosity distributions are almost indistinguishable indicating a high level of completeness, while, for fainter GCs, the sampling of the luminosity function becomes increasingly 

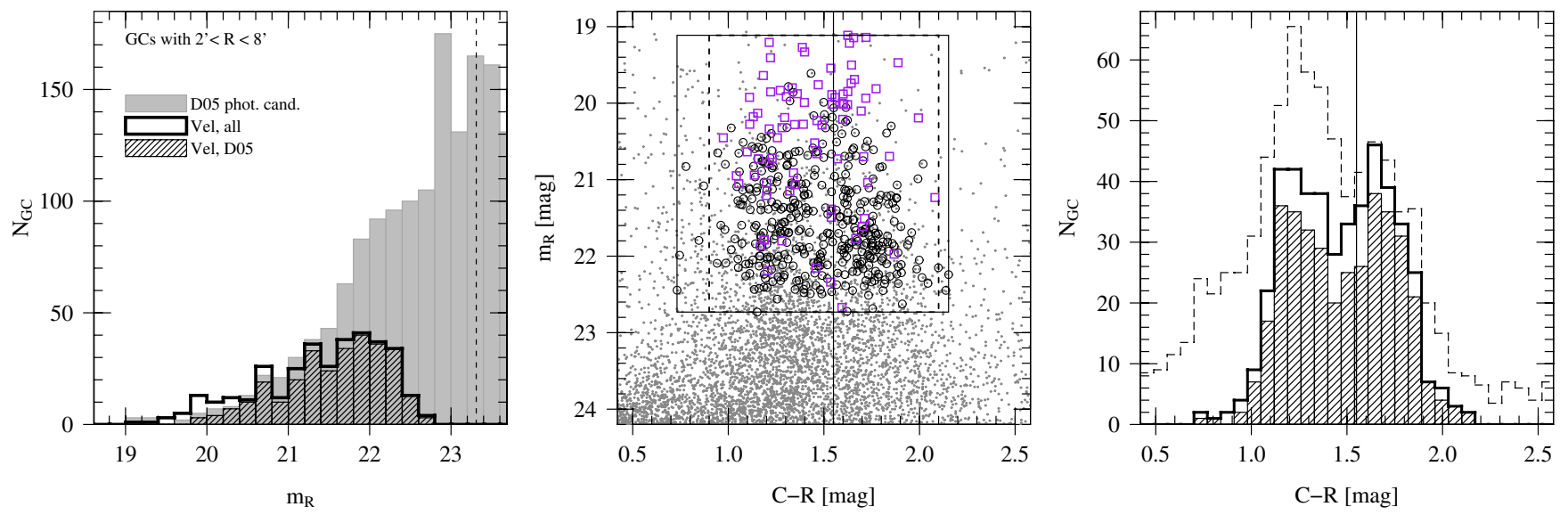

Fig. 5. Photometric properties of the GC sample. Left: luminosity distribution of the GCs in the radial range $2^{\prime}<R<8^{\prime}$ (indicated by the dashed lines in the middle panel of Fig. 4). The grey histogram shows the distribution of the GC candidates from the D+05 catalogue, the dashed bars are those D+05 GCs with velocity measurements. The unfilled histogram (thick black line) shows all GCs with $2^{\prime}<R<8^{\prime}$ and Washington photometry and velocity measurements. The dashed line at $m_{R}=23.33$ mag indicates the turn-over magnitude (TOM) of the GC luminosity function (D+05). Middle: colour-magnitude diagram. Small dots are the point sources from the D+05 catalogue (only objects within the radial range covered by our spectroscopic GC sample are shown), and open symbols are GCs with velocity measurements: squares indicate those GCs with velocity measurements which are not in the $\mathrm{D}+05$ list, circles are those identified in the $\mathrm{D}+05$ catalogue. The solid line at $C-R=1.55$ shows the colour adopted to divide red from blue GCs. The solid rectangle shows the area occupied by the velocity confirmed GCs in this study, and the vertical dashed lines at 0.9 and 2.1 indicate the colour range adopted by D+05 to identify candidate GCs. Right: colour distribution. The unfilled dashed histogram plots the colour distribution of the D+05 candidate GCs with magnitudes and radial distances in the range of our velocity-confirmed GCs. No statistical background has been subtracted; for graphical convenience, the number counts have been scaled by a factor of 0.5. The thick solid line shows the distribution for all velocity-confirmed GCs with Washington photometry, and the dashed histogram shows the colours of the velocity-confirmed GCs with $R \geq 2$ '.5 (cf. Sect. 4.3 for details).

sparse. Note, that even the faintest GCs in the spectroscopic sample are still $\sim 0.6$ mag brighter than the turn-over magnitude $\left(m_{R, \mathrm{TOM}}=23.33, \mathrm{D}+05\right)$.

\subsection{Colour distribution}

The middle panel of Fig. 5 shows the colour-magnitude diagram of the NGC 4636 GCS: open symbols are the GCs from our spectroscopic study. Circles show GCs matched to the D+05 final catalogue, and rectangles are the mostly bright GCs which are not in the D+05 catalogue (cf. Sect. 3.1). Small dots show the objects from the D+05 catalogue (from the radial range covered by the spectroscopically confirmed GCs). The solid rectangle indicates the range of parameters of the GCs with velocities, and the dashed lines at $C-R=0.9$ and 2.1 show the colour interval D+05 used to identify the photometric GC candidates. All but seven of our velocity-confirmed GCs have colours in the range used by $\mathrm{D}+05$, confirming their choice of parameters.

The right panel of Fig. 5 compares the colour histogram for the spectroscopic sample (thick solid line) to the distribution of GC colours from the D+05 photometry (dashed unfilled histogram). Also for the smaller spectroscopic sample, the bimodality is readily visible. The location of the peaks agrees well with the photometric GC candidates. For our sample, the heights of the blue and red peak are very similar, while, for the photometric sample, the blue peak is more prominent. This is due to the fact that the dashed histogram shows all D+05 photometric GC candidates for the full radial interval covered by our study: there is a stronger contribution from larger radii - where blue GCs dominate (see Sect. 10.4) - than for our kinematic sample which becomes increasingly incomplete as one moves away from NGC 4636 (cf. Fig. 4, middle panel).

\subsection{GC colour distribution as a function of galactocentric radius}

Due to the different spatial distributions of metal-poor and metalrich GCs (and the resulting differences with regard to the kinematics), a meaningful dynamical analysis requires a robust partition of the spectroscopic GC sample into blue and red GCs.

$\mathrm{D}+05$ used the minimum $(C-R=1.55)$ of the colour distribution of the GCs in the radial range 3'. $6 \lesssim R \lesssim 8$.'1 to separate the two populations.

We use the model-based mixture modelling software provided in the MCLUST package ${ }^{3}$ (Fraley \& Raftery 2002, 2006) to study the colour distribution of the NGC 4636 spectroscopic GC sample: MCLUST fits the sum of Gaussians (via maximumlikelihood) to the $C-R$ data. The number of Gaussian components fit can either be specified by the user, or MCLUST computes the Bayesian Information Criterion (BIC, Schwarz 1978) to find the optimal number of components.

In Fig. 6, we plot the colours of the velocity-confirmed GCs versus their galactocentric distance (small dots). For a sliding window containing $105 \mathrm{GCs}$, we use MCLUST (in BIC mode) to determine the number of Gaussian components and plot the positions of the peaks as function of radius ${ }^{4}$ (thin solid line). The large data points show the values obtained for the independent bins, and the vertical bars indicate the width(s) of the distribution(s). The mean colour of the GCs is shown as thick solid line. Only for $R \gtrsim 2$ '.5 (dashed vertical line), the colour distribution becomes bimodal.

In Table 3, we list the parameters derived for various subsamples of our data. Unless otherwise indicated, the number

\footnotetext{
3 MCLUST is implemented in the R language and environment for statistical computing and graphics (http://www.r-project.org).

4 The derived quantities are plotted against the lower boundary of the radius interval covered by a bin.
} 
Table 3. Colour distribution of the spectroscopic GC sample from normal mixture modelling.

\begin{tabular}{ccccccccccccc}
\hline \hline ID & & $N$ & Model & $m_{R}$ & $R$ & $N_{b}$ & $\mu_{b}$ & $\sigma_{b}$ & $N_{r}$ & $\mu_{r}$ & $\sigma_{r}$ & $C-R_{\text {divi }}$ \\
$(1)$ & & $(2)$ & $(3)$ & $(4)$ & $(5)$ & $(6)$ & $(7)$ & $(8)$ & $(9)$ & $(10)$ & $(11)$ & $(12)$ \\
\hline 1 & all & 458 & $\mathrm{E}$ & $19.11-22.73$ & $0.51-15.43$ & 226 & 1.25 & 0.16 & 232 & 1.69 & 0.16 & 1.46 \\
2 & $R>2.5$ & 372 & $\mathrm{E}$ & $19.14-22.73$ & $2.53-15.43$ & 183 & 1.26 & 0.15 & 189 & 1.70 & 0.15 & 1.47 \\
3 & $R \leq 2.5$ & 86 & $\mathrm{X}$ & $19.11-22.45$ & $0.51-2.50$ & 86 & 1.42 & 0.26 & $\ldots$ & $\ldots$ & $\ldots$ & $\ldots$ \\
& $R \leq 2.5$ & 86 & $\mathrm{~V}^{\dagger}$ & $19.11-22.45$ & $0.51-2.50$ & 38 & 1.22 & 0.21 & 48 & 1.57 & 0.19 & 1.36 \\
4 & $2.5<R \leq 3.75$ & 86 & $\mathrm{E}$ & $19.64-22.62$ & $2.53-3.72$ & 49 & 1.26 & 0.16 & 37 & 1.72 & 0.16 & 1.50 \\
\hline
\end{tabular}

Notes. ${ }^{(\dagger)}$ Model type and number of components fixed. The parameters were obtained using the MCLUST normal mixture modelling algorithm. The first Column describes and labels the subsets for which the analysis was performed. The total number of GCs is given in Col. 2, the type of model fit to the data is given in Col. 3, where "E" refers to equal-variance (homoscedastic) 2-component models, and "X" to a single-component model. "V" is a heteroscedastic 2-component model. The range of $R$-band magnitudes and radial distances are listed in Cols. 4 and 5 , respectively. Columns 6 through 8 show the number of blue GCs, the position and width of the blue peak. Columns 9 though 11 are the same for the red GCs. Column 12 is the colour dividing blue from red GCs.

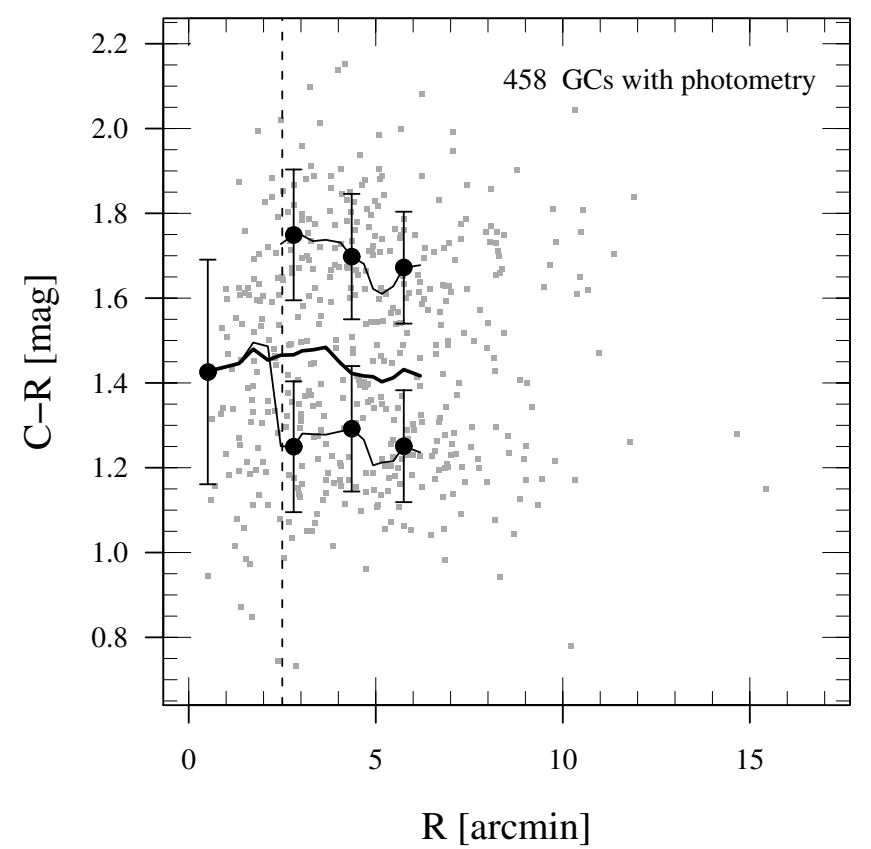

Fig. 6. Colour distribution of the GCs as function of radius. The moving window contains $105 \mathrm{GCs}$, and the data points show the independent bins. The number of components to fit was determined by the mclust software. The "error-bars" give the widths of the respective distributions. The thick solid line shows the colour of the combined light of GCs within the sliding window. The vertical dashed line at 2'.5 $(\simeq 13 \mathrm{kpc})$ indicates the galactocentric distance where the colour distribution becomes bimodal.

of components fit to the data was determined using the BIC. Apart from the GCs with $R<2.5$ all distributions were found to be bimodal.

\subsection{Photometry of the GCs within 2'.5}

As can be seen from Fig. 7, the colour distribution of the GCs with galactocentric distances smaller than 2.5 (sample 3 from Table 3) does not look bimodal, and MCLUST finds the distribution is best described by a single Gaussian (shown as thick solid line). Compared to the GCs with $R>2$.'5 (shown as thick grey curve), the distribution appears to be shifted towards the blue. When fitting a 2-component heteroscedastic model ("V" in Table 3) to the GCs within 2!.5, the fitted distributions (shown as

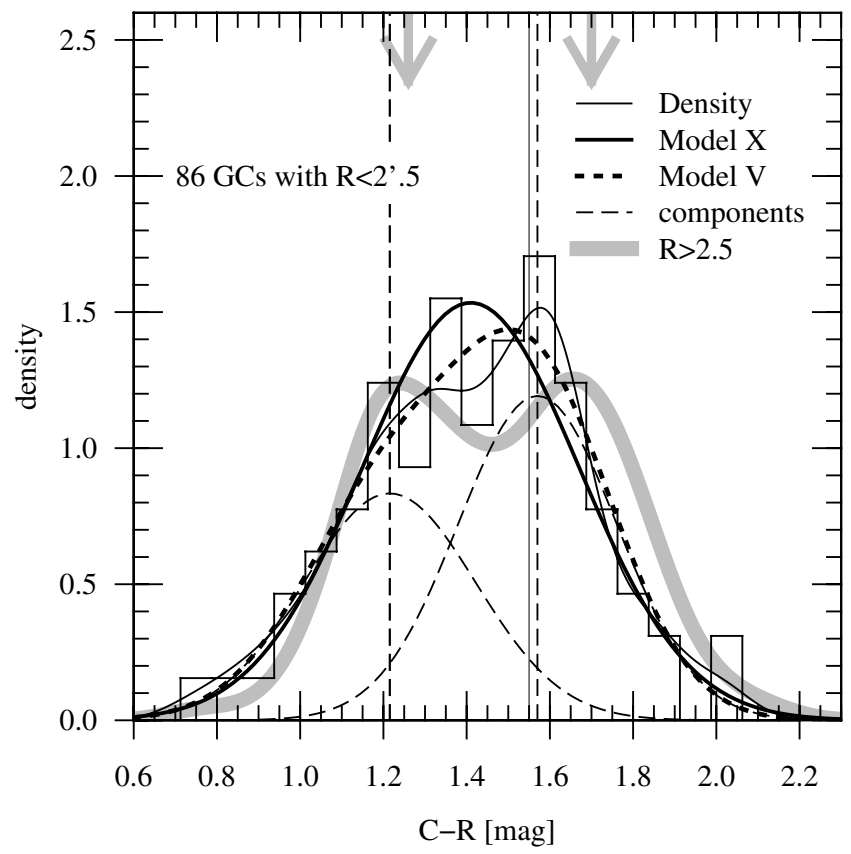

Fig. 7. Colour distribution of the GCs within 2'.5 (subset 3 in Table 3). The thin solid curve is the Gaussian kernel density estimate for a bandwidth of $0.075 \mathrm{mag}$ (same as the bin width of the histogram). The thick solid curve is the one-component model ("X"), and the short-dashed curve is the unequal-variance 2-component model ("V"). The two components are shown with long-dashed curves, the corresponding peaks are indicated by the long-dashed vertical lines. For comparison, the thick grey line shows the kernel density estimate for the colour distribution of the GCs with $R>2.5$ (number 2 in Table 3), and the arrows mark the positions of the peaks. For reference, the colour used to separate blue from red GCs $(C-R=1.55, \mathrm{D}+05)$ is shown as solid vertical line.

thin dashed curves) are broader than for any of the other samples considered, and the peaks are offset to the blue.

To test whether the MCLUST results for the GCs within 2'.5 are due to the small sample size of $86 \mathrm{GCs}$, we apply the algorithm to the $86 \mathrm{GCs}$ in the radial range $2.5<R \leq 3$. 75 (sample 4 in Table 3 ). For this sample of equal size, however, MCLUST determines that the most likely distribution is indeed bimodal with peak positions consistent with those found when considering all GCs outside 2'.5 (sample 2). 
We suspect that the photometry of the point sources within the central 2.5 arcmin is worse than that of sources outside that region because of issues with the subtraction of the galaxy light: the shape of the colour distributions is broadened by larger photometric errors, and a small offset in the continuum subtraction results in a shift towards bluer colours. In the central 2.5 arcmin, it is therefore not possible to separate the blue and red GC populations. Consequently, the dynamical analysis of the subpopulations has to be restricted to galactocentric distances beyond $2.5(\simeq 12.7 \mathrm{kpc})$.

Regarding the spatial distribution and the photometric properties discussed above, we conclude that our spectroscopic sample is a good representation of the GCs surrounding NGC 4636 for galactocentric distances between 2.5 and $8^{\prime}$ $(12.7 \lesssim R \lesssim 40.7 \mathrm{kpc})$.

\section{Definition of the subsamples}

As was demonstrated in Paper I, the presence of interlopers can severely affect the derived line-of-sight velocity dispersion profile and, by consequence the inferred mass profile. In the following paragraphs, we describe our outlier rejection technique. The final subsamples to be used in the dynamical analysis are defined in Sect. 5.2.

\subsection{Interloper rejection}

Objects with velocities that stand out in the velocity vs. galactocentric distance plot (Fig. 3, right panel) are potential outliers. Such "deviant" velocities might be due to measurement errors, a statistical sampling effect, or the presence of an intra-cluster GC population (Bergond et al. 2007; Schuberth et al. 2008). In the low-velocity domain, possible confusion with Galactic foreground stars is the main source of uncertainty.

\subsubsection{Contamination by Galactic foreground stars}

In Sect. 3.3, the division between foreground stars and bona fide NGC 4636 GCs was made at $v=350 \mathrm{~km} \mathrm{~s}^{-1}$ (same as in Paper I). There are, however, seven objects with velocities between 300 and $400 \mathrm{~km} \mathrm{~s}^{-1}$ (shown as labelled white dots in both panels of Fig. 3). In order of decreasing distance from NGC 4636 these are:

1. $7.1: 15\left(v=313 \pm 20 \mathrm{~km} \mathrm{~s}^{-1}, m_{R}=19.83, C-R=0.95\right)$ is almost certainly a foreground star: at a distance of 14.8 we hardly find any NGC 4636 GCs at all (cf. Fig. 3, middle panel). Moreover, this object would be very blue for a GC of this magnitude (cf. Fig. 5, middle panel).

2. f09-57 $\left(v=345 \pm 84 \mathrm{~km} \mathrm{~s}^{-1}, C-R=2.33, m_{R}=22.08\right)$ is probably a foreground star: its very red colour lies outside the range of colours found for the GCs studied in Paper I.

3. Object f09-43 $\left(v=378 \pm 45 \mathrm{~km} \mathrm{~s}^{-1}, m_{R}=20.82\right)$ has an extremely blue colour $(C-R=0.78)$, and its velocity is offset from the NGC $4636 \mathrm{GC}$ velocities found at this radial distance $(R=10 ! 2)$, hence it is most likely a foreground star.

4. f08-13 $\left(v=347 \pm 66 \mathrm{~km} \mathrm{~s}^{-1}, C-R=0.95, R=7 ! 9\right)$ is very blue and quite faint $\left(m_{R}=22.5\right)$.

5. $2.2: 76\left(v=306 \pm 60 \mathrm{~km} \mathrm{~s}^{-1}, C-R=1.40, m_{R}=21.9\right.$, $R=5 ! 3)$ is an ambiguous case.

6. $1.2: 15\left(v=392 \pm 38 \mathrm{~km} \mathrm{~s}^{-1}, C-R=1.70, m_{R}=21.6\right.$, $R=4.9)$ is an ambiguous case.

7. f03-09 $\left(v=350 \pm 69 \mathrm{~km} \mathrm{~s}^{-1}, C-R=1.67, m_{R}=22.2\right.$, $R=4 ! 0)$ is also an ambiguous case.
Thus, three of the seven objects in the velocity range $300-400 \mathrm{~km} \mathrm{~s}^{-1}$ are almost certainly foreground stars because of their unlikely combination of extreme velocities and extreme colour. We remove these objects from the list of GCs. For the remaining four objects (No. 4-7) in this velocity interval, the situation is not as clear, so they remain in the samples to which we apply the outlier rejection scheme described below.

\subsubsection{Outlier rejection algorithm}

In this section, we apply the same outlier rejection method as described in our study of the NGC 1399 GCS (Schuberth et al. 2010) to our data. The method which is based on the tracer mass estimator by Evans et al. (2003) works as follows: for each subsample under consideration, we start by calculating the quantity

$m_{N}=\frac{1}{N} \sum_{i=1}^{N} v_{i}^{2} \cdot R_{i}$,

where $v_{i}$ are the relative velocities of the GCs, and $R_{i}$ their projected galactocentric distances, and $N$ is the number of GCs. Now, we remove the GC with the largest contribution to $m_{N}$, i.e. $\max \left(v^{2} \cdot R\right)$ and calculate the quantity in Eq. (1) for the remaining $N-1 \mathrm{GCs}$, and so on. In the upper panels of Fig. 8 we plot the difference between $m_{j}$ and $m_{j+1}$ against $j$, the index which labels the GCs in order of decreasing $v^{2} \cdot R$.

For the blue GCs (shown in the right panel of Fig. 8) we consider only objects with velocity uncertainties $\Delta v \leq 65 \mathrm{~km} \mathrm{~s}^{-1}$. The function $m_{j}-m_{j+1}$ levels out after the removal of six GCs which are shown as dots in the lower panel of that figure. The thin solid curves enveloping the remaining GCs are of the form

$v_{\max }(R)=\sqrt{\frac{C_{\max }}{R}}$,

where $C_{\max }$ is the product $v^{2} \cdot R$ for the first GC that is not rejected.

For the red GCs, the convergence of $\left(m_{j}-m_{j+1}\right)$ is not as clear. We reject the two objects with low velocities which are most likely foreground stars (objects 6 and 7 from the list in Sect. 5.1.1), and find that the distribution of the remaining red GCs is symmetric with respect to the systemic velocity.

\subsection{The subsamples}

For the analysis of the kinematic properties of the NGC 4636 GCS, we use the following four subsamples:

- Blue All 209 blue GCs with $R \geq 2$ '.5.

- BlueFinal 156 Blue GCs with $R \geq 2.5, \Delta v \leq 65$, six outliers removed.

- Red All 162 red GCs with $R \geq 2$ '.5.

- RedFinal 160 Red GCs with $R \geq 2$ '.5, two outliers removed ${ }^{5}$.

For reference, we compare these to the full sample $(\boldsymbol{A l l})$ of 459 GCs and the sample labelled AllFinal which combines BlueFinal and RedFinal and hence comprises 316 GCs. Details on the line-of-sight velocity distributions (LOSVDs) of these samples are given in the following section.

5 After removing the two outliers from the red GC sample, we find that further restricting the sample objects with velocity uncertainties below $60 \mathrm{~km} \mathrm{~s}^{-1}$ does not significantly change the velocity dispersion profile (cf. Table 6). Therefore, our final red sample still contains 13 GCs with $\Delta v>65 \mathrm{~km} \mathrm{~s}^{-1}$. 

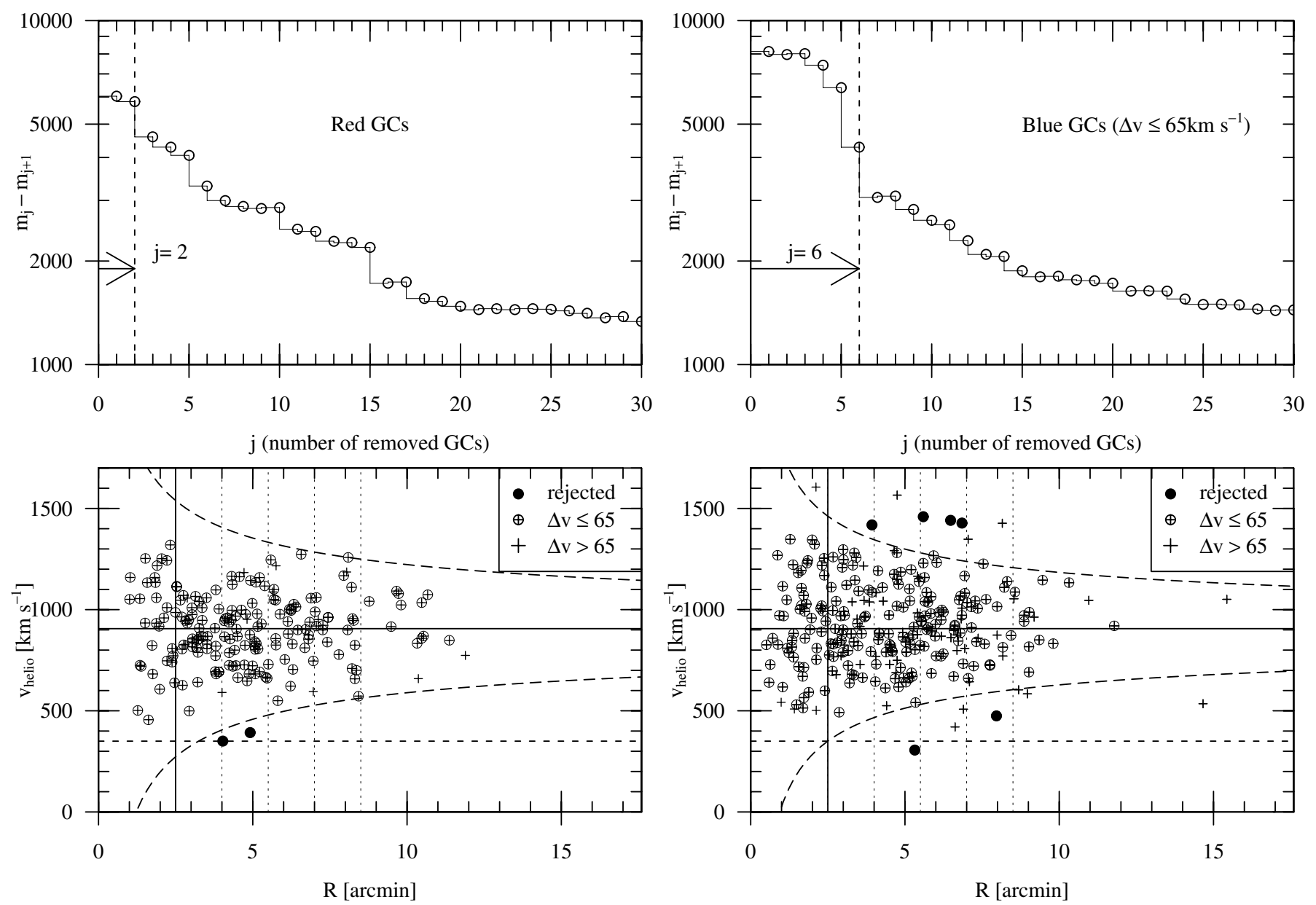

Fig. 8. Outlier removal using the $\max \left(R \cdot v^{2}\right)$ algorithm. Left: all red GCs. In the upper panel, we plot the quantity $\left(m_{j}-m_{j+1}\right)$ against the index $j$. The number of removed GCs $(j=2)$ is indicated by the vertical dashed line and the arrow. In the lower sub-panel, we plot the heliocentric velocities vs. projected radius. Crosses mark GCs with velocity uncertainties $\Delta v>65 \mathrm{~km} \mathrm{~s}^{-1}$, and circles indicate GCs with $\Delta v \leq 65 \mathrm{~km} \mathrm{~s}{ }^{-1}$. The rejected GCs are shown as dots. Right: the same algorithm applied to the Blue GCs with $\Delta v \leq 65 \mathrm{~km} \mathrm{~s}^{-1}$. Six GCs are rejected. In both lower sub-panels, the systemic velocity of NGC 4636 is indicated by a solid horizontal line. The vertical solid line at 2.5 indicates the radius inside which the photometry does not permit a separation of blue and red GCs. The dotted vertical lines show the bins used for the dispersion profiles shown in Fig. 12.

\section{The line-of-sight velocity distribution}

Figure 9 shows the line-of-sight velocity distributions (LOSVDs) for the samples defined above in Sect. 5.2. In each sub-panel, the unfilled histogram shows the respective initial sample, the filled histogram bars show the corresponding final samples. Below we comment on the statistical properties of these distributions which are compiled in Table 4.

\subsection{The Anderson-Darling test for normality}

When adopting $p \leq 0.05$ as criterion for rejecting the Null hypothesis of normality, we find that all subsamples are consistent with being drawn from a normal distribution: the $p$-values returned by the Anderson-Darling test (Stephens 1974) lie in the range $0.15 \leq p \leq 0.72$ (cf. Table 4 Col. 9 ).

\subsection{The moments of the LOSVD}

For all samples, the median value of the radial velocities agrees well with the systemic velocity of NGC $4636\left(906 \pm 7 \mathrm{~km} \mathrm{~s}^{-1}\right.$, Paper I).

\subsubsection{Velocity dispersion}

The velocity dispersion values quoted in Table 4 (Col. 6) were calculated using the expressions given by Pryor \& Meylan (1993), in which the uncertainties of the individual velocity measurements are used as weights.

The velocity dispersion of the final blue sample is $20 \mathrm{~km} \mathrm{~s}^{-1}$ larger than that of the final red sample. Although this difference cannot be considered significant (since the values derived for blue and red GCs marginally agree within their uncertainties), it will be shown below (Sect. 8), that the radial velocity dispersion profiles of the two subpopulations, however, are very different (cf. Figs. 12 and 19).

\subsubsection{Skewness}

The skewness values (Table 4, Col. 7) for the final blue and red samples are consistent with being zero, i.e. the velocity distributions are symmetric with respect to the systemic velocity of NGC 4636. The only (significantly) non-zero skewness is found for the blue GCs prior to weeding out outliers and GCs with large measurement uncertainties. 
Table 4. Statistical properties of the NGC 4636 globular cluster sample.

\begin{tabular}{|c|c|c|c|c|c|c|c|c|}
\hline $\begin{array}{l}\text { ID } \\
\text { (1) }\end{array}$ & Sample & $\begin{array}{l}N_{\mathrm{GC}} \\
(3)\end{array}$ & $\begin{array}{c}\bar{v} \\
(4)\end{array}$ & $\begin{array}{c}\tilde{v} \\
(5)\end{array}$ & $\begin{array}{c}\sigma \pm \Delta \sigma \\
(6)\end{array}$ & $\begin{array}{l}\text { Skew } \\
(7)\end{array}$ & $\begin{array}{c}\kappa \\
(8)\end{array}$ & $\begin{array}{c}p(\mathrm{AD}) \\
(9)\end{array}$ \\
\hline All & & 459 & 916 & 909 & $197 \pm 7$ & $0.17 \pm 0.12$ & $0.05 \pm 0.23$ & 0.15 \\
\hline AllFinal & $R \geq 2.5 \& \Delta v \leq 65 \&$ OutRej & 387 & 920 & 912 & $181 \pm 7$ & $0.11 \pm 0.09$ & $-0.45 \pm 0.13$ & 0.15 \\
\hline Blue & $C-R \leq 1.55 \& R \geq 2.5$ & 209 & 928 & 912 & $198 \pm 11$ & $0.30 \pm 0.15$ & $0.03 \pm 0.28$ & 0.31 \\
\hline BlueFinal & $C-R \leq 1.55 \& R \geq 2.5 \& \Delta v \leq 65 \&$ OutRej & 156 & 925 & 912 & $173 \pm 10$ & $-0.10 \pm 0.12$ & $-0.69 \pm 0.18$ & 0.17 \\
\hline Red & $C-R>1.55 \& R \geq 2.5$ & 162 & 893 & 900 & $162 \pm 10$ & $-0.03 \pm 0.20$ & $0.12 \pm 0.40$ & 0.64 \\
\hline RedFinal & $C-R>1.55 \& R \geq 2.5 \&$ OutRej & 160 & 900 & 901 & $153 \pm 10$ & $-0.03 \pm 0.14$ & $-0.48 \pm 0.20$ & 0.72 \\
\hline
\end{tabular}

Notes. Columns (1) and (2) label the samples, and the number of GCs is given in (3). Columns (4) and (5) list the mean and median radial velocity (in $\mathrm{km} \mathrm{s}^{-1}$ ). The velocity dispersion in $\left(\mathrm{km} \mathrm{s}^{-1}\right)$ as computed with the Pryor \& Meylan (1993) formula is given in Col. (6). Columns (7) and (8) give the skewness and the reduced kurtosis, the uncertainties were estimated using a bootstrap with 999 resamplings. The $p$-value returned by the Anderson-Darling test for normality is given in Col. (9).

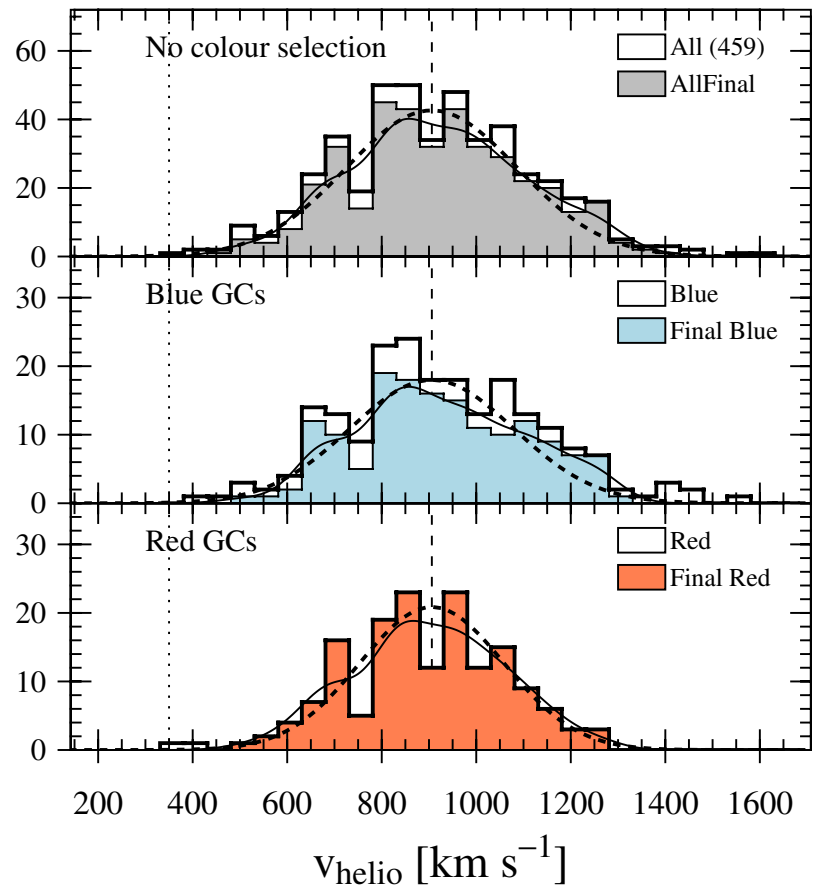

Fig. 9. NGC 4636 line-of-sight velocity distributions. From top to bottom, the panels show the velocity distribution for the entire sample, the blue, and the red subsample, as defined in Sect. 5.2. In all panels, the vertical dashed line shows the systemic velocity of NGC 4636. The unfilled histograms show the respective velocity distributions prior to any quality selection and outlier rejection. The filled histograms show the final samples. In all panels, the dashed curve shows the Gaussian corresponding to the dispersion value for the respective final sample as given in Table 4. The thin solid line is the corresponding Gaussian kernel density estimate (for a bandwidth of $50 \mathrm{~km} \mathrm{~s}^{-1}$, same as the bin width).

\subsubsection{Kurtosis}

Column 8 of Table 4 lists the reduced kurtosis (i.e. a Gaussian distribution has $\kappa=0$ ) of the respective subsamples. The fourth moment of the LOSVD reacts quite severely to the treatment of extreme velocities: the removal of only two clusters from the Red sample changes the kurtosis from $\kappa=0.12 \pm 0.40$ to $\kappa=-0.48 \pm 0.20$ (RedFinal). The FinalBlue sample also has a negative kurtosis, meaning that both distributions are more "flat-topped" than a Gaussian. Indeed, the kernel density estimates (thin solid lines in Fig. 9) have somewhat broader wings and a flatter peak than the corresponding Gaussians (thick dotted curves). These differences, however, are quite subtle and we suspect that the samples considered in this work are probably still too small to robustly determine the 4th moment of the velocity distributions. The slightly negative kurtosis values are, however, consistent with isotropy (a projected kurtosis of zero is expected only for the isothermal sphere).

We conclude that the final GC samples that will be used for the dynamical analysis are symmetric with respect to the systemic velocity of NGC 4636 and do not show any significant deviations from normality.

\section{Rotation}

Due to the very inhomogeneous angular coverage of the data, the results from the search for rotation of the NGC 4636 GCS presented in Paper I were quite uncertain. Below, we use our enlarged data set for a re-analysis and compare the findings to the ones in Paper I. To detect signs of rotation, we fit the following relation to the data:

$v_{r}(\Theta)=v_{\text {sys }}+A \sin \left(\Theta-\Theta_{0}\right)$,

where $v_{r}$ is the measured radial velocity at the azimuth angle $\Theta$, $A$ is the amplitude (in units of $\mathrm{km} \mathrm{s}^{-1}$ ), and $\Theta_{0}$ is the position angle of the axis of rotation (see Côté et al. 2001, for a detailed discussion of the method).

In Table 5, we present the results of the rotation analysis for the different subsamples of our data: Cols. 2 and 3 give the values of $\Theta_{0}$ and the amplitude $A$ found for the samples presented in Table 4 . Columns 4 to 6 give the corresponding results obtained for the GCs in the radial range $2.5<R<8$ '.0, where the spatial completeness of our sample is highest (cf. Sect.4.1). It appears that the rotation signal (within the uncertainties) is robust with respect to the radial range considered and the application of the outlier rejection algorithm.

To search for variations of the rotation signal with galactocentric radius, we plot, in the left and middle panel of Fig. 10, the rotation parameters $\Theta_{0}$ and $A$ determined for moving bins of $50 \mathrm{GCs}$. The following paragraphs summarise the results for blue and red GCs.

\subsection{Rotation of the blue GCs}

Table 5 shows that the rotation signature is strongest for the blue GCs (final sample).

In the right panel of Fig. 10, we plot the radial velocities against the position angle for the final blue sample restricted to galactocentric distances below $8^{\prime}$ (which, in Table 5 is the sample with the strongest rotation signature). The data for the 

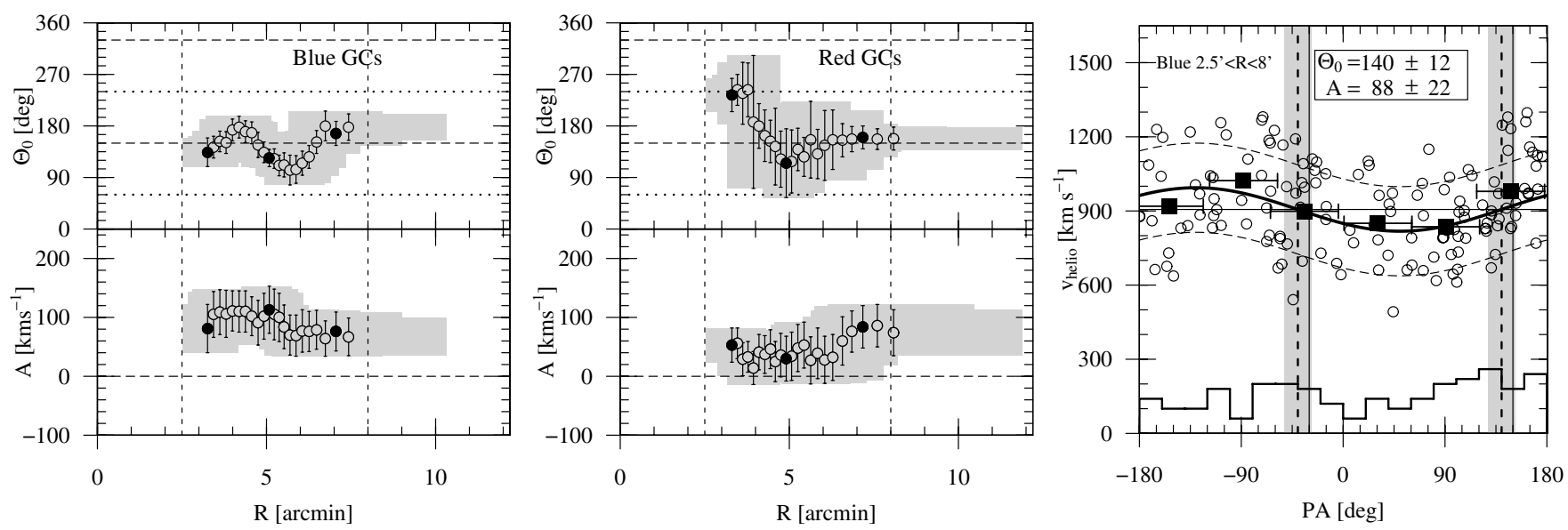

Fig. 10. Rotation of the NGC 4636 globular cluster system. Left: circles show the rotation of the final blue sample (BlueFinal) computed for moving bins of $50 \mathrm{GCs}$ with a step site of $5 \mathrm{GCs}$. The grey area indicates the radial coverage and the uncertainties. Dots indicate independent bins. The upper sub-panel shows the rotation angle $\Theta_{0}$ vs. projected galactocentric radius. The NGC 4636 photometric major and minor axis are shown as long-dashed and dotted horizontal lines, respectively. The lower sub-panel shows the amplitude $A$ as function of radius. Middle: the same as the left panel but for the final red sample (RedFinal). In both plots, the dashed vertical lines indicate the radial range $2.5<R<8^{\prime}$ for which we have the best spatial coverage of the GCS (see also Sect. 4.1). Right: velocity versus position angle for the 141 blue GCs (circles) from the sample BlueFinal with distances between 2.5 and $8^{\prime}$. The thick solid curve shows the best fit of Eq. (3) to the data, the thin dashed curves are offset by the sample dispersion $\left(\sigma=180 \mathrm{~km} \mathrm{~s}^{-1}\right)$. The vertical dashed and solid lines show the best-fit $\Theta_{0}$ and the photometric major axis of NGC 4636, respectively. The grey areas indicate the uncertainty $\Delta \Theta_{0}$. The squares show the mean velocity for bins of $60^{\circ}$. The unfilled histogram at the bottom shows the angular distribution of the GCs (the bins have a width of $20^{\circ}$ and the counts are multiplied by a factor of 20 for graphic convenience).

Table 5. Rotation of the NGC 4636 globular cluster system.

\begin{tabular}{lccccc}
\hline \hline & & & \multicolumn{3}{c}{$2.5 \leq R \leq 8^{\prime}$} \\
\cline { 4 - 6 } Sample ID & $\Theta_{0}$ & $A$ & $N_{\mathrm{GC}}$ & $\Theta_{0}$ & $A$ \\
& {$[\mathrm{deg}]$} & {$\left[\mathrm{km} \mathrm{s}^{-1}\right]$} & & {$[\mathrm{deg}]$} & {$\left[\mathrm{km} \mathrm{s}^{-1}\right]$} \\
$(1)$ & $(2)$ & $(3)$ & $(4)$ & $(5)$ & $(6)$ \\
\hline All & $160 \pm 21$ & $34 \pm 14$ & 325 & $152 \pm 16$ & $49 \pm 16$ \\
All.Final & $150 \pm 15$ & $46 \pm 15$ & 269 & $141 \pm 12$ & $59 \pm 15$ \\
Blue & $156 \pm 18$ & $58 \pm 21$ & 185 & $144 \pm 17$ & $66 \pm 23$ \\
Blue.Final & $144 \pm 13$ & $77 \pm 21$ & 141 & $140 \pm 12$ & $88 \pm 22$ \\
Red & $172 \pm 27$ & $37 \pm 19$ & 138 & $175 \pm 35$ & $32 \pm 20$ \\
Red.Final & $171 \pm 26$ & $36 \pm 18$ & 136 & $174 \pm 34$ & $30 \pm 19$ \\
\hline
\end{tabular}

Notes. The position angle of NGC 4636 is $150^{\circ}$. The first column gives the sample identifier (Sect. 5.2). Columns 2 and 3 give the axis of rotation and the amplitude, respectively. Columns 4 through 6 give the number of GCs, rotation angle and amplitude for the samples restricted to radii $R \leq 8^{\prime}$ (i.e. the range in which the completeness of the spectroscopic GC sample is large).

141 GCs are shown as circles, and the squares mark the mean velocity calculated for $60^{\circ}$ wide bins. The thick solid line shows Eq. (3) $\left(A=88 \mathrm{~km} \mathrm{~s}^{-1}\right.$ and $\left.\Theta_{0}=140^{\circ}\right)$.

\subsection{Rotation of the red GCs}

For the red GC samples, the overall rotation signature as quoted in Table 5 is weaker than that of the blue GCs. Within $R \lessgtr 6$ ' $^{\prime}$, the amplitude for the final sample plotted in the middle panel of Fig. 10 is consistent with being zero (104 GCs with 2 !.05 $\lesssim$ $\left.R \lesssim 66^{\prime} 0: A=17 \pm 22 \mathrm{~km} \mathrm{~s}^{-1}, \Theta_{0}=186 \pm 73^{\circ}\right)$. Only the last few bins suggest an increase with the amplitude reaching values of $\sim 80 \mathrm{~km} \mathrm{~s}^{-1}$ (32 GCs with 6! $0 \lesssim R \lesssim 8^{\prime} .0: A=82 \pm 37 \mathrm{~km} \mathrm{~s}^{-1}$, $\Theta_{0}=160 \pm 21^{\circ}$ ).

Between $3^{\prime}$ and $5^{\prime}$, the axis of rotation changes from being aligned with the minor axis to the photometric major axis of NGC 4636. Given the low values of the amplitude at these radii, however, this change of the axis of rotation remains uncertain. For radii beyond $5^{\prime}$, the axis of rotation remains constant and coincides with the major axis.

\subsection{Comparison to Paper I}

The findings in this section deviate significantly from the values presented in Paper I, where no rotation was detected for the blue GCs, while the strongest signal was found for the red clusters within $4^{\prime}\left(\Theta_{0}=72 \pm 25^{\circ}, A=-144 \pm 44 \mathrm{~km} \mathrm{~s}^{-1}\right)$.

The discrepant findings are likely due to the azimuthal incompleteness of the data in Paper I.

\section{Globular cluster velocity dispersion profiles}

Figure 12 shows the velocity dispersion profiles obtained for the blue (upper panel) and red (lower panel) subsamples defined in Sect. 5.2. The GC velocities were grouped into six radial bins (shown as dotted lines in the lower panels of Fig. 8). The first bin comprises the GCs within 2.5, and the following four bins (starting at $2.5,4.0,5.5$ and 7 '.0 $^{\prime}$ have a width of 1.5. The outermost bin $(8.5<R \leq 15.5)$ collects the GCs in the more sparsely populated (and sampled) outer GCS. The data are given in Table 6. Circles are the values obtained for the Blue and Red subsamples and dots represent the dispersion profiles determined for the final samples (BlueFinal and RedFinal). The values from Paper I (Table 4 therein) are shown as diamonds.

\subsection{Blue GCs}

Compared to the initial sample, the removal of GCs with velocity uncertainties $\Delta v \geq 65 \mathrm{~km} \mathrm{~s}^{-1}$ and the six probable interlopers identified in Sect. 5.1.2 significantly reduces the velocity dispersion, in particular in the 4th and 5th bin. The values for the final blue sample (dots) agree very well with the data from Paper I (diamonds). The dispersion profile of the blue sample declines with galactocentric radius. 
Table 6. Velocity dispersion profiles for fixed radial bins.

\begin{tabular}{|c|c|c|c|c|c|c|c|c|c|}
\hline \multirow[b]{2}{*}{$\begin{array}{l}\mathrm{N}^{\mathrm{o}} \\
(1)\end{array}$} & \multirow[b]{2}{*}{$\begin{array}{r}\text { range } \\
\text { (2) }\end{array}$} & \multicolumn{2}{|r|}{ Blue } & \multicolumn{2}{|c|}{ BlueFinal } & \multicolumn{2}{|r|}{ Red } & \multicolumn{2}{|c|}{ RedFinal } \\
\hline & & $\begin{array}{c}n \\
\text { (3) }\end{array}$ & $\begin{array}{c}\sigma \\
(4)\end{array}$ & $\begin{array}{l}n \\
\text { (5) }\end{array}$ & $\begin{array}{c}\sigma \\
(6)\end{array}$ & $\begin{array}{l}n \\
\text { (7) }\end{array}$ & $\begin{array}{c}\sigma \\
(8)\end{array}$ & $\begin{array}{l}n \\
(9)\end{array}$ & $\begin{array}{c}\sigma \\
(10)\end{array}$ \\
\hline 1 & $00^{\prime} 0-2{ }^{\prime} .5$ & 56 & $249 \pm 25$ & 52 & $226 \pm 23$ & 30 & $235 \pm 32$ & 30 & $235 \pm 32$ \\
\hline 2 & $2 \cdot .5-4^{\prime} .0$ & 55 & $199 \pm 21$ & 44 & $195 \pm 22$ & 45 & $140 \pm 16$ & 45 & $140 \pm 45$ \\
\hline 3 & $4^{\prime} 0-5 \cdot .5$ & 60 & $200 \pm 20$ & 45 & $186 \pm 21$ & 50 & $183 \pm 21$ & 48 & $154 \pm 48$ \\
\hline 4 & $5 \cdot 5-7: 0$ & 51 & $200 \pm 23$ & 38 & $146 \pm 21$ & 32 & $169 \pm 23$ & 32 & $169 \pm 32$ \\
\hline 5 & $7.0-8.5$ & 25 & $206 \pm 32$ & 18 & $154 \pm 28$ & 22 & $165 \pm 27$ & 22 & $165 \pm 22$ \\
\hline 6 & $8.5-15^{\prime} .5$ & 18 & $166 \pm 33$ & 11 & $131 \pm 34$ & 13 & $122 \pm 31$ & 13 & $122 \pm 13$ \\
\hline
\end{tabular}

Notes. Column 1 gives the bin number and Col. 2 is the radial range covered by a given bin. Column 3 is the number of GCs in the Blue subsample, Col. 4 the line-of-sight velocity dispersion in $\mathrm{km} \mathrm{s}^{-1}$. Columns 5 and 6 are the same for the BlueFinal sample. The corresponding values for the red GCs are given in Cols. 7 through 10. Columns 11 and 12 show the values obtained when further restricting the RedFinal sample to velocities with uncertainties below $3 \leq 65 \mathrm{~km} \mathrm{~s}^{-1}$.

Table 7. GC Velocity dispersion profiles for constant number bins.

\begin{tabular}{cccc|ccl}
\hline \hline \multicolumn{6}{c}{ Blue GCs (BlueFinal) } & \multicolumn{3}{|c}{ Red GCs (RedFinal) } \\
No. & $\bar{R}$ & $\sigma \pm \Delta \sigma$ & $n$ & $\bar{R}$ & $\sigma \pm \Delta \sigma$ & $n$ \\
$(1)$ & $(2)$ & $(3)$ & $(4)$ & $(5)$ & $(6)$ & $(7)$ \\
\hline & $1 ! 20$ & $208 \pm 30$ & 26 & $\ldots$ & $\ldots$ & $\ldots$ \\
& $2 ! 03$ & $242 \pm 35$ & 26 & $1 ! 85$ & $235 \pm 31$ & 30 \\
\hline 1 & $3 ! 05$ & $202 \pm 24$ & 39 & $3 ! 13$ & $135 \pm 16$ & 40 \\
2 & $4 \cdot 55$ & $179 \pm 21$ & 39 & $4 ! 44$ & $152 \pm 19$ & 40 \\
3 & $5 ! 75$ & $162 \pm 20$ & 39 & $5 ! 81$ & $172 \pm 21$ & 40 \\
4 & $7 ! 92$ & $143 \pm 19$ & 39 & $8 ! 48$ & $151 \pm 19$ & 40 \\
\hline
\end{tabular}

Notes. The first column numbers the bins (for GCs with $R>2.5$ ). Column 2 gives the mean galactocentric distance of the blue GCs, Col. 3 gives the velocity dispersion in units of $\mathrm{km} \mathrm{s}^{-1}$, and Col. 4 is the number of blue GCs in that bin. Columns 5 through 8 give the corresponding values for the red GCs. For completeness, the first two rows give the values for GCs inside 2.5 where the colour distribution is not bimodal.

\subsection{Red GCs}

The velocity dispersion profile of the red GCs (cf. Fig. 12, lower panel) shows a more complex behaviour. The sudden drop by almost $100 \mathrm{~km} \mathrm{~s}^{-1}$ from the first to the second bin was already present in the data for Paper I. While the poorer data in Paper I still left the possibility of a sampling effect, our new enlarged data shows the same feature which we attribute to the fact that blue and red GCs cannot be separated within the central 2.5: inside this radius, the presence of a substancial number of "contaminating" metal-poor GCs (which would have a broader velocity distribution) within our "red" sample dominates the measurement and leads to the very high dispersion value.

To search for colour trends amongst the GCs inside 2.5, we plot in Fig. 11 (upper panel), the the line-of-sight velocity dispersion as a function of colour. Dots represent the 82 GCs within 2.5 with velocity uncertainties below $65 \mathrm{~km} \mathrm{~s}^{-1}$. The $316 \mathrm{GCs}$ from the Final sample are shown as squares. Inside 2.5, there is no discernible trend with colour, except for a dip near the colour used to divide blue from red GCs. For GCs outside 2.5, we observe a constant dispersion for the red GCs, which then increases towards bluer colours. However, there is no sign of a "jump" near the dividing colour as seen in the NGC 1399 GCS (Schuberth et al. 2010). For GCs with projected galactocentric distances between 2.5 and 4.0, we find a low dispersion of only $140 \pm 16 \mathrm{~km} \mathrm{~s}^{-1}$. This value then again rises to almost $170 \mathrm{~km} \mathrm{~s}^{-1}$ between 5.5 and 8'0.

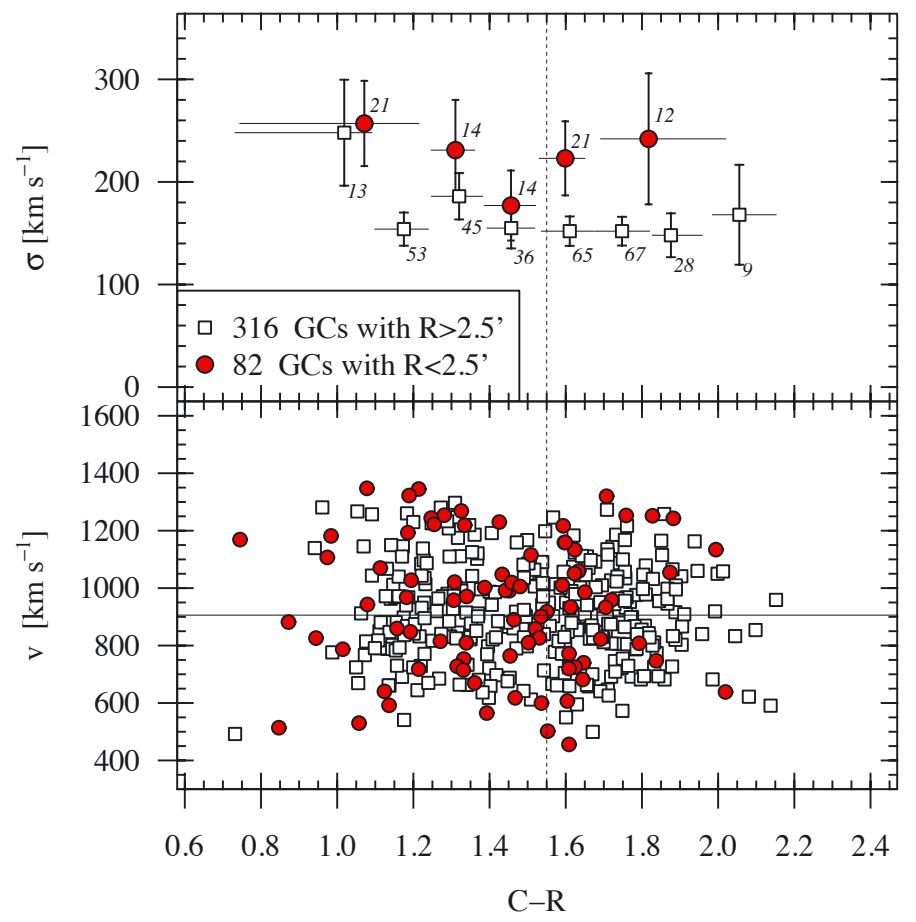

Fig. 11. Upper panel: line-of-sight velocity dispersion as a function of colour. Dots represent the values for the $82 \mathrm{GCs}$ within 2.5 and velocity uncertainties below $65 \mathrm{~km} \mathrm{~s}^{-1}$, squares show the results for the $316 \mathrm{GCs}$ of the AllFinal sample. The labels give the number of GCs per bin. The bin size is $0.15 \mathrm{mag}$, but the bluest and reddest bins have been resized so that no objects are excluded. The horizontal bars mark the colour range covered by a given bin. Lower panel: heliocentric velocity as a function of colour. The symbols are the same as in the upper panel.

\section{NGC 4636 stellar kinematics}

Long slit spectra of NGC 4636 were obtained by Bender et al. (1994), and Kronawitter et al. (2000) presented detailed modelling based on these data. Recently, Pu \& Han (2011) presented radial velocities and velocity dispersions derived from deep long-slit spectra along the major and minor axis. Their data reach out to $\sim 2 ! 3$, corresponding to about $11.5 \mathrm{kpc}$.

We will use the stellar kinematics to constrain the halo models derived for the GCs (see Sect. 11.1.3). Below, in Fig. 13, we show the Bender et al. (1994) data and compare them to our own measurements obtained in parallel with the GC observations. 


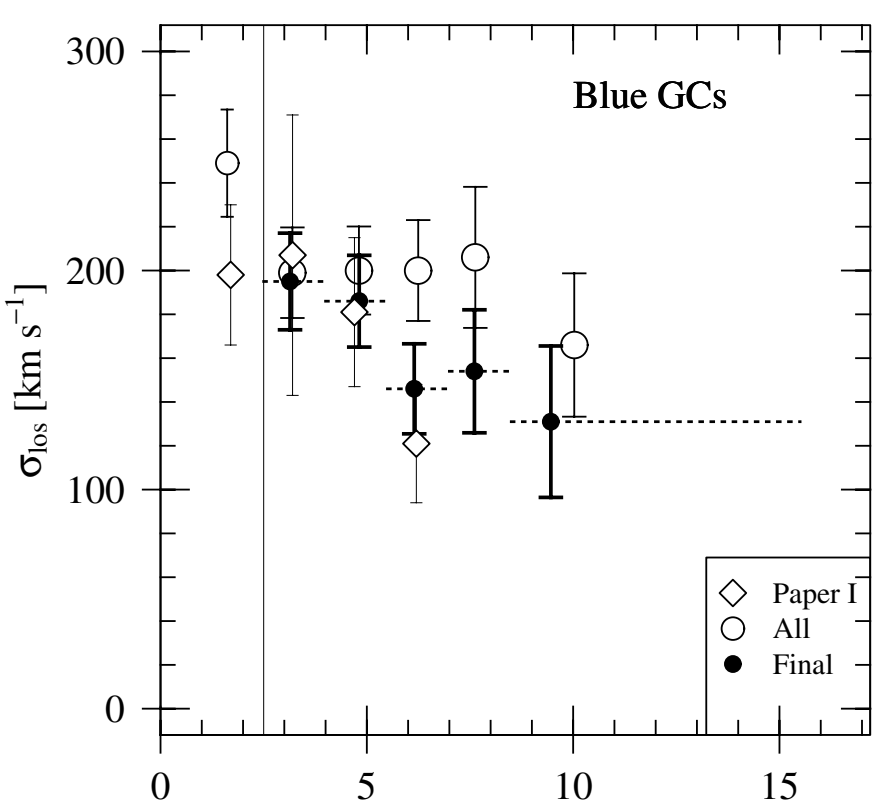

$\mathrm{R}$ [arcmin]

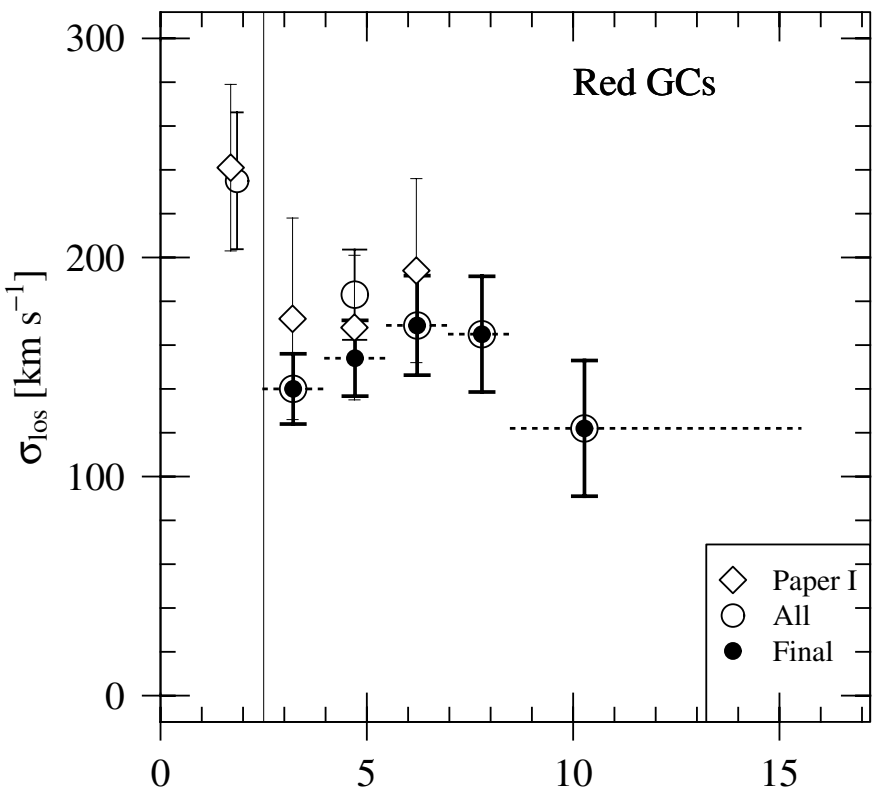

$\mathrm{R}$ [arcmin]

Fig. 12. Velocity dispersion profiles. Upper panel: circles show the dispersion values for the blue GCs (for fixed radial bins cf. Table 6) prior to quality selection and outlier removal. Dots are the values for the final blue sample. The dashed horizontal "error bars" indicate the radial range of a given bin; the bins used here are the ones indicated by the dotted lines in the lower panels of Fig. 8. The dispersion values from Paper I are shown as diamonds. In both panels, the vertical line at 2.5 indicates the first bin which covers the radius inside which blue and red GCs cannot be distinguished. Lower panel: the same for the red GCs.

\subsection{FORS 2 spectra of NGC 4636}

During the first MXU observations of the NGC 4636 GCs, we placed 29 slits (along the North-South direction) on NGC 4636 itself. This mask (Mask 1_1 from Paper I) has a total exposure time of $2 \mathrm{~h}(7200 \mathrm{~s})$, and the final image is the co-addition of

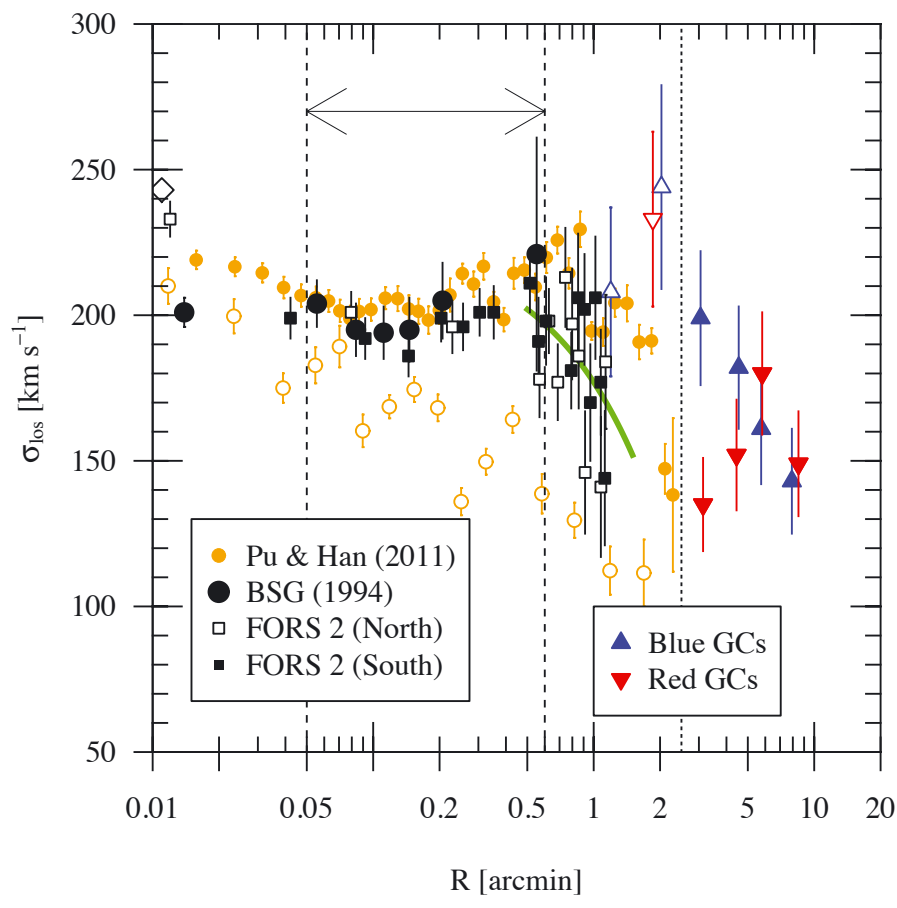

Fig. 13. The stellar velocity dispersion profile of NGC 4636. The diamond is the central dispersion measured by Proctor \& Sansom (2002). Large dots show the values from Bender et al. (1994), squares are the values derived from our FORS 2 spectra (see text for details). Unfilled (filled) squares indicate slits North (South) of the galaxy centre. The vertical dashed lines indicate the region we use to model the galaxy's velocity dispersion profile (cf. Sect. 11.1.3). The solid green curve is a linear fit to the data for $R>0,5$. The major axis measurements by $\mathrm{Pu} \&$ Han (2011) are shown as small (orange) dots. For radii $\leq 0.219^{\prime}$, where the long slit extends to either side of the galaxy, we plot the averaged values; the uncertainties were added in quadrature. For comparison, the data points for blue and red GCs for constant number bins (final samples, values from Table 7) are shown as triangles. The vertical dotted line at $R=2.5$ marks the radius inside which blue and red GCs cannot be separated.

three consecutive exposures, thus ensuring a good cosmic-ray rejection. The data were reduced in the same manner as the slits targeting GCs. The sky was estimated from the combination of several sky-slits located $\sim 3^{\prime}$ from the centre of NGC 4636.

Using the pPXF (penalised PiXel Fitting) routine by Cappellari \& Emsellem (2004), we determined the line-of-sight velocity dispersion for the one-dimensional spectra. The uncertainties were estimated via Monte Carlo simulations in which we added noise to our spectra and performed the analysis in the same way as with the original data. The templates used in the analysis were taken from the Vazdekis (1999) library of synthetic spectra. Our data are shown in Fig. 13 where unfilled and filled squares refer to slits placed to the North and South of the centre of NGC 4636, respectively. The data are listed in Table A.1.

\subsection{The stellar velocity dispersion profile of NGC 4636}

In Fig. 13, we compare our results (squares) with the values from Bender et al. (1994) (large dots). Within the radial range 0'04 $R \lessgtr 0$. 6 , the agreement between both data sets is excellent.

For the central velocity dispersion (measured from a $2^{\prime \prime}$ long slit within about $1^{\prime \prime}$ of the centre of NGC 4636), we find $\sigma_{0}=233 \pm 6 \mathrm{~km} \mathrm{~s}^{-1}$. This is substantially higher than the value 
published by BSG94 $\left(\sigma_{0}=211 \pm 7 \mathrm{~km} \mathrm{~s}^{-1}\right)$. However, the high central velocity dispersion we find is supported by the measurement published by Proctor \& Sansom (2002) who found $\sigma_{0}=$ $243 \pm 3 \mathrm{~km} \mathrm{~s}^{-1}$ (shown as diamond in the right panel of Fig. 13). The low central dispersion quoted by BSG94 is likely due to the instrumental setup: these authors used a slit of 2 .' 1 width, and at this spatial resolution the luminosity weighted dispersion measured for the centre may be substantially lower than values obtained using a smaller slit width: Proctor \& Sansom used a slit of 1".25 which provides a similar spatial resolution as our 1'. 0 wide MXU slits, yielding similar dispersion values.

For the dynamical modelling, dispersion values at large radial distances are of particular interest. Unfortunately, due to the low $\mathrm{S} / \mathrm{N}$ in the small slits we used, the quality of our data degrades for radial distances beyond $R \gtrsim 0$.'7: the uncertainty of the individual data points increases and so does the scatter. However, the velocity dispersion seems to decline as indicated by the solid curve in Fig. 13 (right panel) which shows a linear fit to $\sigma_{\text {los }}(R)$ for $R>0.5$. This trend is confirmed by the recently published measurements by Pu \& Han (2011) (small dots in Fig. 13) which clearly show a declining stellar velocity dispersion for radii beyond $\sim 0.6(\approx 3 \mathrm{kpc})$. These data have a higher $\mathrm{S} / \mathrm{N}$ than our FORS measurements and extend out to $2 ! 3$, i.e. almost into the regime where GC dynamics is available for both the metal-poor and the metal-rich subpopulation (shown as filled triangles in Fig. 13). In this context, it is interesting to note that the very low velocity dispersion of $135 \pm 16 \mathrm{~km} \mathrm{~s}^{-1}$, observed for the red GCs near $\sim 3$ '. 1 is consistent with the outermost stellar velocity dispersion value of $138 \pm 26 \mathrm{~km} \mathrm{~s}^{-1}$. This might suggest a connection between stars and metal-rich GCs similar to the one reported for NGC 1399 Schuberth et al. (2010).

For the dynamical modelling of the stellar component of NGC 4636 we use the Bender et al. (1994) data in the radial range 0.05 to $0.6(0.25-3.1 \mathrm{kpc})$, indicated by the dashed lines in the right panel of Fig. 13. The central data points are not included in our modelling since the deprojection of the luminosity profile and, by consequence, the stellar mass profile are only reliable for $R \gtrsim 100 \mathrm{pc} \simeq 0$.'2 (see Sect. 10.2).

\section{Jeans models for NGC 4636}

In the next paragraphs, we give the relevant analytical expressions and outline how we construct the spherical, non-rotating Jeans models for NGC 4636.

In Paper I, we chose an NFW-halo (Navarro et al. 1997) to represent the dark matter in NGC 4636. In this work, we will consider both NFW halos and two mass distribution with a finite central density: the cored profile proposed by Burkert (1995) which has the same asymptotic behaviour as the NFW halo and the logarithmic potential which leads to (asymptotically) flat rotation curves.

\subsection{The Jeans equation and the line-of-sight velocity dispersion}

The spherical, non-rotating Jeans equation (see e.g. Binney \& Tremaine 1987) reads:

$\frac{\mathrm{d}\left(n(r) \sigma_{r}^{2}(r)\right)}{\mathrm{d} r}+2 \frac{\beta(r)}{r} n(r) \sigma_{r}^{2}(r)=-n(r) \frac{G \cdot M(r)}{r^{2}}$,

with $\quad \beta \equiv 1-\frac{\sigma_{\theta}^{2}}{\sigma_{r}^{2}}$.
Here, $r$ is the radial distance from the centre and $n$ is the spatial (i.e., three-dimensional) density of the GCs; $\sigma_{r}$ and $\sigma_{\theta}$ are the radial and azimuthal velocity dispersions, respectively. $\beta$ is the anisotropy parameter, $M(r)$ the enclosed mass (i.e. the sum of stellar and dark matter) and $G$ is the constant of gravitation.

For our analysis, we use the expressions given by, e.g. Mamon \& Łokas (2005), see also van der Marel \& Franx (1993). Given a mass distribution $M(r)$, a three-dimensional number density of a tracer population $n(r)$, and a constant anisotropy parameter $\beta$, the solution to the Jeans equation (Eq. (4)) reads:

$n(r) \sigma_{r}^{2}(r)=\mathrm{G} \int_{r}^{\infty} n(s) M(s) \frac{1}{s^{2}}\left(\frac{s}{r}\right)^{2 \beta} \mathrm{d} s$.

This expression is then projected using the following integral:

$\sigma_{\mathrm{los}}^{2}(R)=\frac{2}{N(R)}\left[\int_{R}^{\infty} \frac{n \sigma_{r}^{2} r \mathrm{~d} r}{\sqrt{r^{2}-R^{2}}}-R^{2} \int_{R}^{\infty} \frac{\beta n \sigma_{r}^{2} \mathrm{~d} r}{r \sqrt{r^{2}-R^{2}}}\right]$,

where $N(R)$ is the projected number density of the tracer population, and $\sigma_{\text {los }}$ is the line-of-sight velocity dispersion, to be compared to our observed values. In the following, we discuss the quantities required to determine $\sigma_{\mathrm{los}}(R)$.

\subsection{Luminous matter}

To assess the stellar mass of NGC 4636 we need to deproject the galaxy's surface brightness profile. Moreover, to consistently model the line-of-sight velocity dispersion profile of the stars (cf. Fig. 13), we require analytical expressions for both, the projected and the three-dimensional stellar density.

As in Paper I, we use the data published by D+05 (shown as dots in the upper panel of Fig. 14), for which the authors gave the following fit:

$$
\begin{aligned}
& \mu(R)= \\
& -2.5 \log \left(3.3 \times 10^{-7}\left(1+\frac{R}{0^{\prime} .11}\right)^{-2.2}+5.5 \times 10^{-9}\left(1+\frac{R}{8^{\prime} .5}\right)^{-7.5}\right) .
\end{aligned}
$$

Their fit is shown as dashed line in Fig. 14. There is, however, no analytical solution to the deprojection integral for this function. We therefore fit the data using the sum of three Hubble-Reynolds profiles instead:

$\mu(R)=-2.5 \log \left(\sum_{i=1}^{3} N_{0, i}\left[1+\left(\frac{R}{R_{0, i}}\right)^{2}\right]^{-\alpha_{i}}\right)$,

where the parameters are given in Table 8. Our fit is shown as solid black line in Fig. 14, and the thin gray lines indicate the three components. The deprojection of Eq. (8) reads:

$j(r)\left[\frac{L_{\odot}}{\mathrm{pc}^{3}}\right]=\sum_{i=1}^{3} \frac{N_{0, i}^{\prime}}{R_{0, i}^{\prime} \mathcal{B}\left(\frac{1}{2}, \alpha_{i}\right)}\left[1+\left(\frac{r}{R_{0, i}^{\prime}}\right)^{2}\right]^{-\left(\alpha_{i}+1 / 2\right)}$.

Where $\mathcal{B}$ is the Beta function and $N^{\prime}{ }_{0, i}=C_{M_{R}} \cdot N_{0, i}$, where $C_{M_{R}}=$ $2.192 \times 10^{10}$ is the factor converting the surface brightness into units of $L_{\odot} \mathrm{pc}^{-2}$ for $M_{\odot, R}=4.28$. The radii are in pc, i.e. for a distance of $17.5 \mathrm{Mpc} R_{0, i}^{\prime}=5.09 \times 10^{3} \cdot R_{0, i}$.

The lower panel of Fig. 14 compares the deprojection as given in Eq. (9) (solid black line) to the curve obtained by numerically deprojecting Eq. (7). Within the radius interval covered by the data points, both deprojections agree extremely well, 


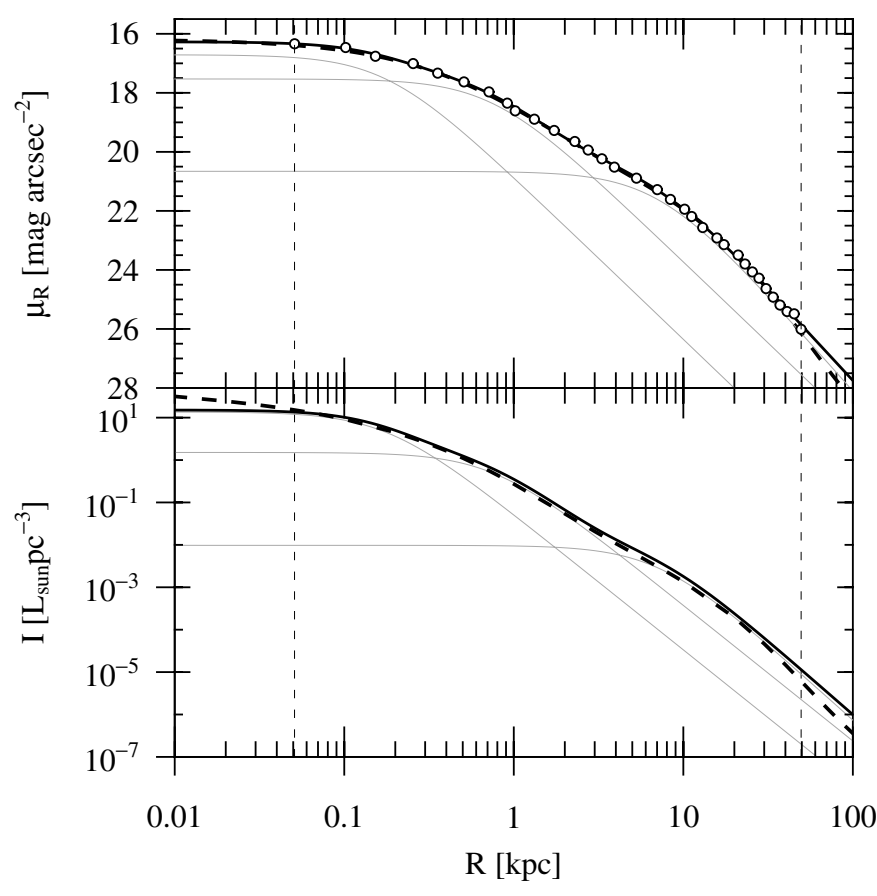

Fig. 14. Deprojecting the surface brightness profile of NGC 4636. Upper sub-panel: $R$-band surface brightness profile. The data points are from D05+ (their Table A.4), and the dashed line is the fit given by $\mathrm{D}+05$ (Eq. (7)). The solid line is the three-component fit given in Eq. (8), and the thin solid lines show the individual components. Bottom sub-panel: luminosity density profiles in units of $L_{\odot} \mathrm{pc}^{-3}$. The dashed line was obtained by numerically integrating Eq. (7). The solid line is the analytical deprojection of Eq. (8); again, the components are shown as thin solid lines. The vertical dotted lines indicate the radial range of the data points shown in the upper panel.

Table 8. Fit parameters for the luminosity density profile of NGC 4636 and the GC number density profiles.

\begin{tabular}{lclcc}
\hline \hline & $N_{0}$ & \multicolumn{1}{c}{$R_{0}$} & $\alpha$ & $\mathcal{B}\left(\frac{1}{2}, \alpha\right)$ \\
\hline Lumprof 1 & $2.07 \times 10^{-7}$ & $3.47 \times 10^{-2}$ & 1.1 & 1.887 \\
Lumprof 2 & $9.73 \times 10^{-8}$ & $1.47 \times 10^{-1}$ & 1.1 & 1.887 \\
Lumprof 3 & $5.45 \times 10^{-9}$ & 1.41 & 1.3 & 1.708 \\
\hline Blue & $12.0 \pm 1.1$ & $6.0 \pm 0.3$ & $2.0 \pm 0.1$ & $\frac{4}{3}$ \\
Red & $12.4 \pm 1.1$ & $6.8 \pm 0.3$ & $3.0 \pm 0.1$ & $\frac{16}{15}$ \\
\hline
\end{tabular}

Notes. The first three rows give the parameters for the three-component Hubble-Reynolds profile fit to the luminosity density profile (Eq. (8)). The last two rows are the parameters found for the GCs (Eq. (11)).

and we proceed to use the analytical expression given in Eq. (9) to represent the density distribution of the stars in NGC 4636.

The stellar mass profile is then obtained through integration:

$M(r)=\Upsilon_{\star, R} \cdot 4 \pi \int_{0}^{r} j(s) s^{2} \mathrm{~d} s$

where $\Upsilon_{*, R}$ is the $R$-band mass-to-light ratio of the stellar population of this galaxy (see below in Sect. 10.3).

Since the integral in Eq. (10) cannot be expressed in terms of simple standard functions, we use an approximation in our calculations: the inner part ( $r \lesssim 45 \mathrm{kpc}$ ) is represented by a sequence of polynomials, while the behaviour at larger radii is well represented by an arctan function. The stellar mass profile is

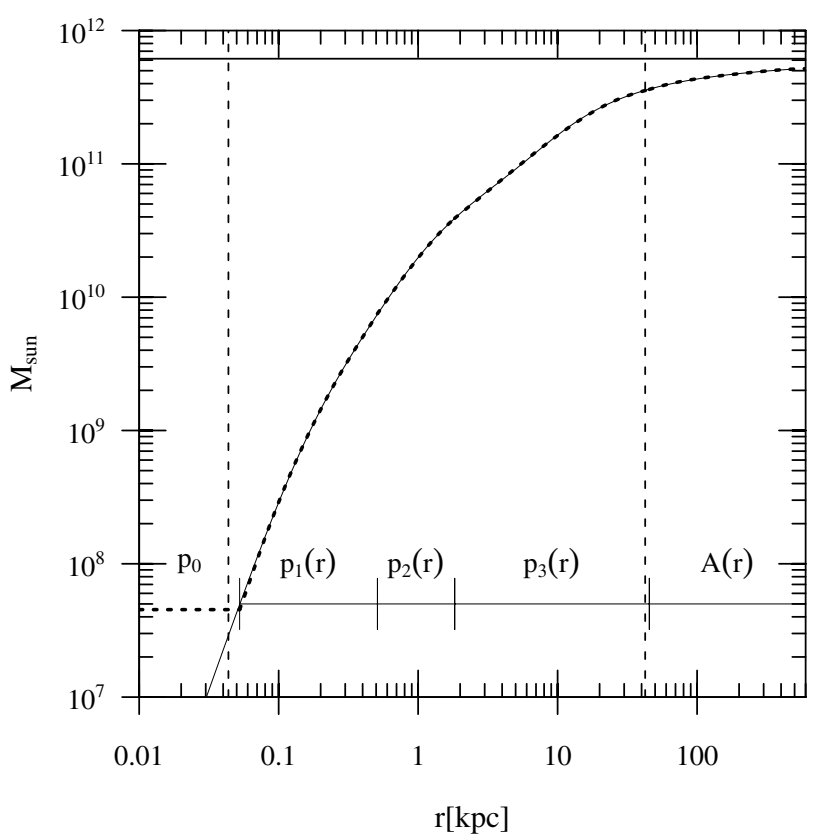

Fig. 15. NGC 4636 stellar mass profile. The thin solid curve is Eq. (10) for $\Upsilon_{\star, R}=5.8$ (obtained through numerical integration). The thick dotted curve is the piecewise approximation used in our modelling. The radial range of the respective pieces is indicated by the bars, and the functions and coefficients are given in Appendix B. The vertical dashed lines indicate the radial range of the photometric data by $\mathrm{D}+05$. The horizontal line at $6.15 \times 10^{11} M_{\odot}$ shows the asymptotic value, i.e. $M(r=\infty)$.

plotted in Fig. 15, and the expressions and coefficients are given in Appendix B.

\subsection{The stellar mass-to-light ratio}

In Paper I, we used an $R$-band $\Upsilon_{\star}=6.8$. This was derived from the dynamical estimate for the $B$-band given by Kronawitter et al. (2000). Having adopted a distance of $17.5 \mathrm{Mpc}$ for our current analysis, the value from Paper I which was based on a distance of $15 \mathrm{Mpc}$ is reduced to $\Upsilon_{\star, R}=5$.8. We will adopt this value for our dynamical modelling of the NGC 4636 GCs.

\subsection{Globular cluster number density profiles}

Below we present the fits to the number density profiles of the GC subpopulations as listed in Table A3 of D+05 and the analytical expressions for the deprojections. As in our study of the NGC 1399 GCS (Schuberth et al. 2010) we parametrise the two-dimensional number density profiles in terms of a Reynolds-Hubble law:

$N(R)=N_{0}\left(1+\left(\frac{R}{R_{0}}\right)^{2}\right)^{-\alpha}$,

where $R_{0}$ is the core radius, and $2 \cdot \alpha$ is the slope of the powerlaw in the outer region. For the above expression, the Abel inversion has an analytical solution and the three-dimensional number density profile reads:

$\ell(r)=\frac{N_{0}}{R_{0}} \frac{1}{\mathcal{B}\left(\frac{1}{2}, \alpha\right)} \cdot\left(1+\left(\frac{r}{R_{0}}\right)^{2}\right)^{-\left(\alpha+\frac{1}{2}\right)}$,

where $\mathcal{B}$ is the Beta function. For both subpopulations, the fits are performed for the radial range $2.5 \leq R \leq 13.5$ where the 
Y. Schuberth et al.: Dynamics of the NGC 4636 globular cluster system. II.

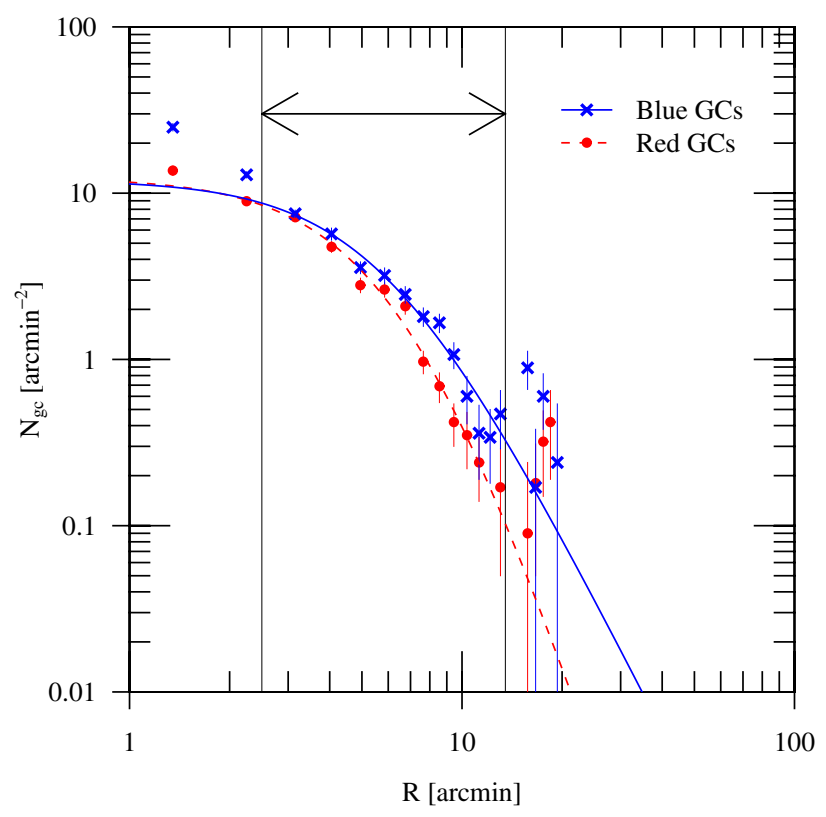

Fig. 16. Number density profile of the blue and red GCs. The data from D+05 (their Table A3) are shown as crosses and dots for the blue and red GCs, respectively. The curves are the fits (cf. Eq. (11), Table 8) to the data, and the radial range $2.5 \leq R \leq 13.5$ is indicated by the arrow and the vertical lines.

lower boundary is the minimum radius where blue and red GCs can be separated. The upper boundary corresponds to the radius where the GC counts reach the background level (D+05). The parameters obtained for the blue and red GCs are given in Table 8. Figure 16 shows the data and the fitted profiles. Note that the profile of the red GCs is significantly steeper than that of the blue GCs.

\subsection{The dark matter halo}

All three dark matter halos considered in this study have two free parameters, allowing a direct comparison of the results.

\subsubsection{The NFW profile}

The mass profile of the NFW halo reads:

$M_{\mathrm{NFW}}(r)=4 \pi \varrho_{\mathrm{s}} r_{\mathrm{s}}^{3} \cdot\left(\ln \left(1+\frac{r}{r_{\mathrm{s}}}\right)-\frac{\frac{r}{r_{\mathrm{s}}}}{1+\frac{r}{r_{\mathrm{s}}}}\right)$,

where $\varrho_{\mathrm{s}}$ and $r_{\mathrm{s}}$ are the characteristic density and scale radius, respectively.

To express the halo parameters in terms of concentration and virial mass, we use the definitions from Bullock et al. (2001) and define the virial radius $R_{\text {vir }}$ such that the mean density within this radius is $\Delta_{\text {vir }}=337$ times the mean (matter) density of the universe (i.e. $0.3 \rho_{c}$ ), and the concentration parameter is defined as $c_{\mathrm{vir}}=R_{\mathrm{vir}} / r_{\mathrm{s}}$.

\subsubsection{The Burkert halo}

The density profile for the cored halo which Burkert (1995) introduced (to represent the dark matter halo of dwarf galaxies) reads:

$\varrho(r)=\frac{\varrho_{0}}{\left(1+\frac{r}{r_{0}}\right)\left(1+\frac{r^{2}}{r_{0}^{2}}\right)}$, and the cumulative mass is given by the following expression:

$$
\begin{aligned}
& M(r)= \\
& 4 \pi \varrho_{0} r_{0}^{3}\left(\frac{1}{2} \ln \left(1+\frac{r}{r_{0}}\right)+\frac{1}{4} \ln \left(1+\frac{r^{2}}{r_{0}^{2}}\right)-\frac{1}{2} \arctan \left(\frac{r}{r_{0}}\right)\right) .
\end{aligned}
$$

\subsubsection{The logarithmic potential}

The logarithmic potential (see Binney 1981; Binney \& Tremaine 1987), by construction, yields (asymptotically) flat rotation curves. In contrast to the NFW profile, it has a finite central density. The spherical logarithmic halo has two free parameters, the asymptotic circular velocity $v_{0}$, and a core radius $r_{0}$. The mass profile reads:

$M_{\mathrm{Log}}(r)=\frac{1}{G} \cdot \frac{r \cdot v_{0}^{2}}{1+\left(\frac{r_{0}}{r}\right)^{2}}$,

where $G$ is the constant of gravitation.

\subsection{Modelling the velocity dispersion profiles}

To find the parameters that best describe the observed GC velocity dispersion data, we proceed as described in Schuberth et al. (2010). For a given tracer population and anisotropy $\beta \in\{-0.5,0,+0.5\}$, we create a grid of models where the density (or $v_{0}$ in case of the logarithmic halo) acts as free parameter while the radii have discrete values, i.e. $r_{\text {dark }} \in$ $\{1,2,3, \ldots, 100\} \mathrm{kpc}$. For each point of this grid, the line-of-sight velocity dispersion (Eq. (6)) is computed using the expressions given in Mamon \& Łokas (2005), where the upper limit of the integral in Eq. (6) is set to $600 \mathrm{kpc}$.

To find the joint solution for the different tracer populations (labelled $a$ and $b$ ), we determine the combined parameters by minimising the sum $\chi^{2}=\chi_{a}^{2}+\chi_{b}^{2}$. The confidence level (CL) contours are calculated using the definition by Avni (1976), i.e. using the difference $\delta \chi^{2}$ above the minimum $\chi^{2}$ value. With two free parameters, e.g. $\left(r_{\text {dark }}, \rho_{\text {dark }}\right)$ the 68,90 , and 99 per cent contours correspond to $\chi^{2}=2.30,4.61$, and 9.21, respectively. The results for the three dark matter halos (NFW, Burkert and logarithmic potential) are presented in the following section.

\section{The mass profile of NGC 4636}

We model the observed line-of-sight dispersions for the final samples of the red and blue GCs shown in Fig. 19 and listed in Table 7 for three different parametrisations of the dark halo.

\subsection{Jeans models for an NFW halo}

\subsubsection{NFW Halo: results for the blue GCs}

In Fig. 17, we show the NFW models for the blue GCs after transforming the parameters to the $\left(M_{\mathrm{vir}}, c_{\mathrm{vir}}\right)$ plane using the definitions in Bullock et al. (2001). In all panels, the respective best fit value as given in Table 9 is shown as a cross. Circles are the corresponding values from Paper I. In all cases, these values lie within the $68 \%$ CL contour of the present study.

From the very elongated shape of the confidence level contours it is apparent that while the GCs can be used to estimate the total mass of the halo, the concentration is only poorly constrained. As will be shown in Sect. 11.4.1, this degeneracy can be 

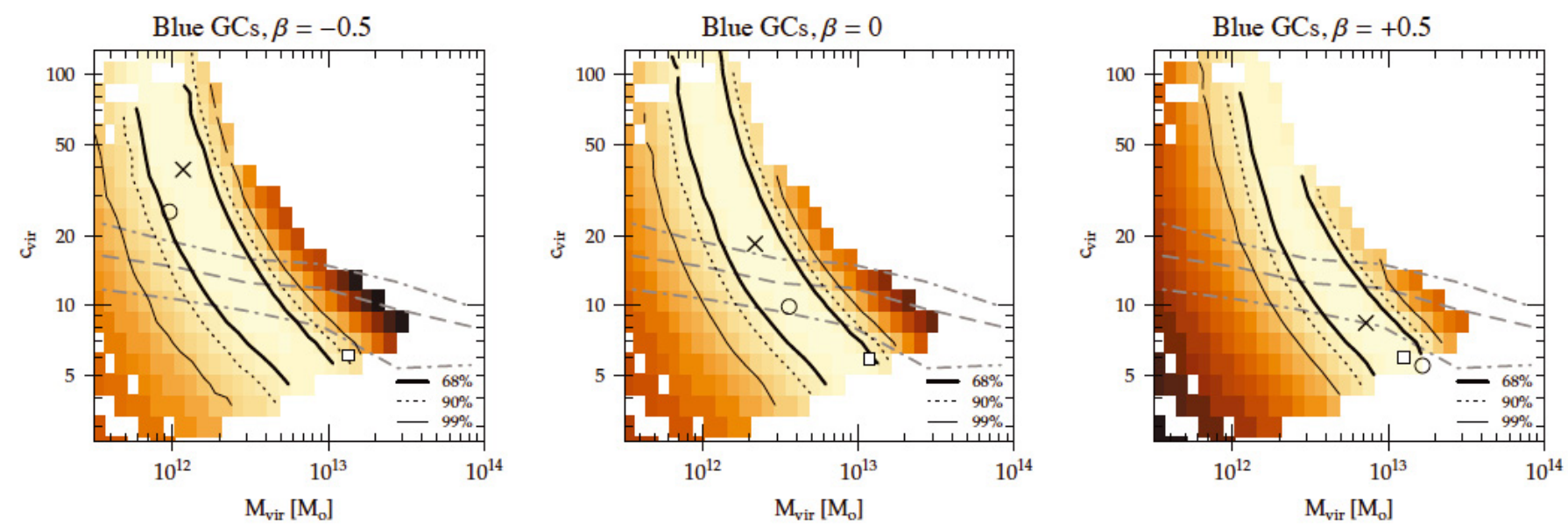

Fig. 17. Jeans models for the blue GCs (BlueFinal) for an NFW-type dark halo. From left to right, the panels show the results for $\beta=-0.5,0$ and +0.5 . The parameters are shown in the $\left(M_{\mathrm{vir}}, c_{\mathrm{vir}}\right)$-plane. The thick solid, dashed and thin solid lines indicate the 68,90, and 99 per cent confidence limits. The colour map is the same for all panels, and the cross indicates the location of the minimum $\chi^{2}$ value. The square shows the minimum $\chi^{2}$ for the joint models obtained from the blue GCs and an isotropic model for the NGC 4636 stellar dispersion profile. The circle indicates the respective best-fit value from Paper I. All model parameters are listed in Table 9. In all panels, the long-dashed (dash-dotted) lines show the median (68 per cent values) for simulated NFW halos as found by Bullock et al. (2001).

partially overcome by considering models for the stellar velocity dispersion profile of NGC 4636.

The best-fit dispersion profiles for the three values of the anisotropy parameters $(\beta \in\{-0.5,0,+0.5\})$ are shown as thin black lines in the left panel of Fig. 19 and the corresponding parameters of the NFW halos are listed in Table 9 (Cols. 4-9). For all three values of $\beta$, a very good agreement between data and models can be achieved, and the differences between the $\chi^{2}$ values is marginal. The models diverge at small radii $(R \lessgtr 2 ! 5 \approx$ $13 \mathrm{kpc}$ ) where the velocity dispersion and the shape of the number density profile of the blue GCs cannot be well constrained. For comparison, we plot (as dot-dashed line) the velocity dispersion curve expected if there were no dark matter and the only mass were that of the stars.

As expected, the best-fit halo derived assuming a tangential orbital anisotropy $(\beta=-0.5$, B.tan, long-dashed line) is less massive than the one obtained in the isotropic case (B.iso, solid line) and the model for a radial bias $(\beta=+0.5$, B.rad, shortdashed line) returns the most massive dark halo. This is also illustrated by the bottom left panel of Fig. 19 where the corresponding mass profiles (thin black lines) are shown in terms of the circular velocity.

\subsubsection{NFW halo: results for the red GCs}

The resulting halo parameters for the red clusters are listed in Table 9 (Cols. 4-9) and illustrated in the middle panel of Fig. 19. Here, the agreement between data and models is worse than in the case of the blue GCs. A considerable part of the uncertainty is caused by the curiously low value at $\sim 16 \mathrm{kpc}$. In spite of this, the resulting circular velocities (shown as thick grey lines in the bottom left of Fig. 19) of the different halo models are not dramatically different from those of the blue GCs. For all three values of $\beta$, the circular velocity stays approximately constant within $40 \mathrm{kpc}$.

\subsubsection{Model for the stars}

We use the stellar velocity dispersion measurements presented by Bender et al. (1994) to constrain the concentration parameter

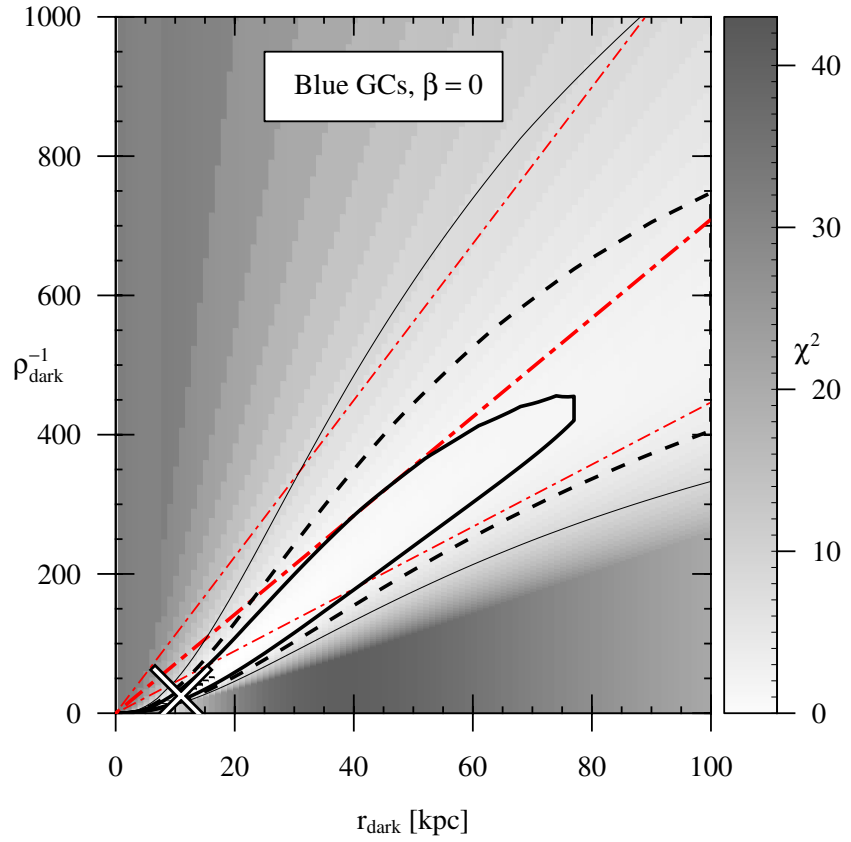

Fig. 18. Jeans models for the blue GCs (sample BlueFinal, $\beta=0$ ) for a Burkert dark matter halo. The cross marks the best-fit parameters (cf. Table 9). The thick solid, dashed and thin solid lines indicate the 68, 90 and 99 per cent confidence limits. The thick dot-dashed line indicates the Donato et al. (2009) central dark matter surface density relation $\log \mu_{0 D}=2.15 \pm 0.2\left[\log \left(M_{\odot} \mathrm{pc}^{-2}\right)\right]$ (the thin dot-dashed lines show the uncertainties).

of the NFW halo. The right panel of Fig. 20 shows the $\left(M_{\mathrm{vir}}-\right.$ $c_{\text {vir }}$ ) plane for the isotropic case. High concentrations are excluded, since adding large amounts of dark matter in the central parts of NGC 4636 would severely overestimate the velocity dispersion profile of the stellar component.

\subsection{Jeans models for a Burkert halo}

Figure 18 shows the parameter space explored to find the bestfit isotropic Jeans model for the blue GCs for a Burkert-type 
Y. Schuberth et al.: Dynamics of the NGC 4636 globular cluster system. II.

Table 9. NGC 4636 Jeans modelling best-fit NFW profiles, Burkert halos and logarithmic potentials.

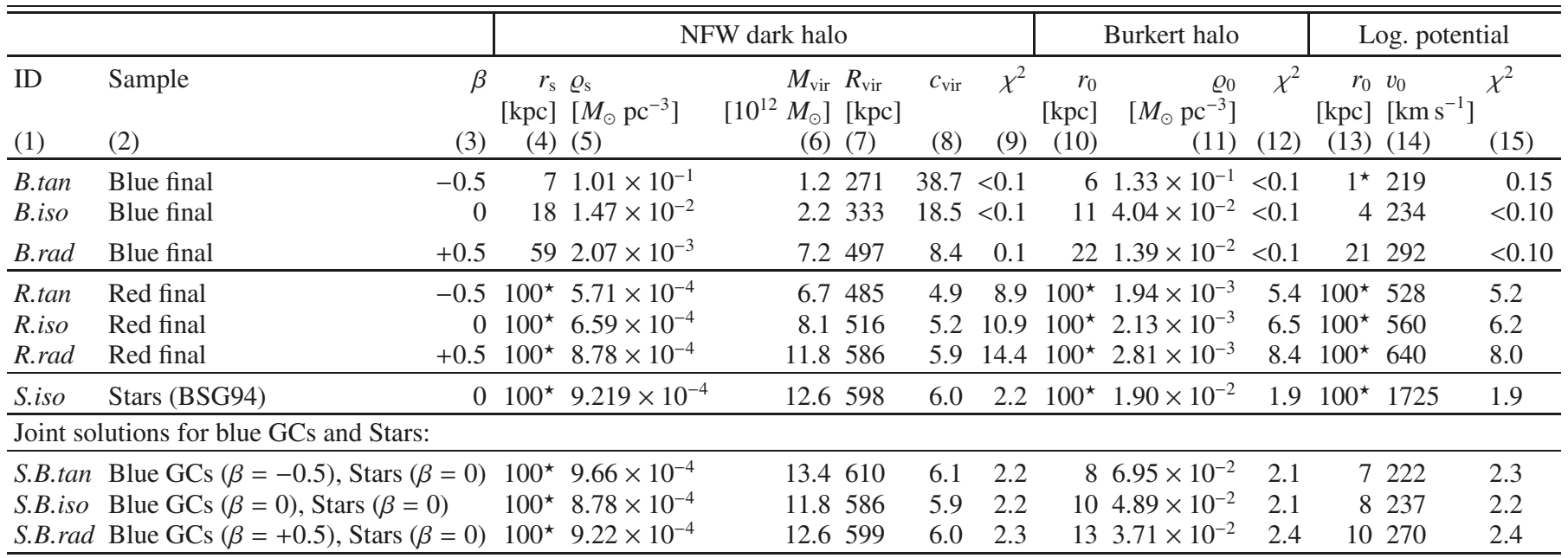

Notes. In all models, the $R$-band stellar mass-to light ratio is $\Upsilon_{\star, R}=5.8$. Column (1) labels the models. The second column specifies the dispersion profile to which the Jeans models are fit. Column (3) gives the anisotropy parameter $\beta$. Columns (4)-(8) list the NFW (Eq. (13)) and virial parameters (see text for details). Column (9) gives the $\chi^{2}$ value. The parameters for the Burkert (1995) halos (15), i.e. $r_{0}, \varrho_{0}$ and the $\chi^{2}$ value of the best fit model are given in Cols. (10)-(12). Columns (13)-(15) are the values for the logarithmic potential (Eq. (16)). Asterisks indicate that the corresponding value is located at the edge of the model grid.

dark halo. The best-fit Burkert models for the GCs are shown in the middle panels of Fig. 19, and the parameters are given in Cols. 11-12 of Table 9. The circular velocities corresponding to the different mass distributions are compared in the bottom middle panel of Fig. 19. The discrepancies between the bestfit models for the blue GCs (shown as thin black lines) and the models for the red GCs (thick grey curves) do not permit to prefer any specific halo model.

\subsection{Jeans models for a logarithmic potential}

The results are summarised in Table 9, and the model grids solutions in the $\left(r_{0}, v_{0}\right)$-plane for the blue GCs (for $\beta=-0.5$ and 0 ) are shown in Fig. 21. Again, one notes a strong degeneracy: the asymptotic velocity $v_{0}$ (and hence the total mass) is well constrained, while the scale radius $r_{0}$ is not.

\subsection{Joint solutions}

To find a joint solution describing the velocity dispersion profiles of the three tracer populations, we combine the $\chi^{2}$ values of the corresponding models and obtain the solution by finding the minimum in the co-added $\chi^{2}$ maps (cf. Sect. 10.6).

\subsubsection{Models for the blue GCs and the stellar velocity dispersion profile}

Since the best agreement between models and data can be achieved for the blue GCs and the stellar velocity dispersion profile (see Fig. 19 and the $\chi^{2}$-values given in Table 9), we will first combine these two tracer populations to obtain a joint model. For the blue GCs, the anisotropy parameters $\beta$ takes the values $-0.5,0,+0.5$, while the stellar models are isotropic. The parameters for corresponding joint models (labelled S.B.tan, S.B.iso and S.B.rad) are given in Table 9. The best-fit joint (isotropic) models are shown in the right panel of Fig. 20 (lower sub-panel). The agreement between data and model is best for the two cored halo parametrisations: the velocity dispersions of both the stars and the blue GCs are very well reproduced by a Burkert halo with $\rho_{0}=4.89 \times 10^{-2} M_{\odot} \mathrm{pc}^{-3}$, $r_{0}=10 \mathrm{kpc}$ or a spherical logarithmic potential with $r_{0}=8 \mathrm{kpc}$ and $v_{0}=237 \mathrm{~km} \mathrm{~s}^{-1}$. The best-fit joint NFW halo, on the other hand, has a very large scale radius and over-estimates the velocity dispersion of the blue GCs in the last bin (although model and data still agree within the uncertainties).

\section{Discussion}

\subsection{Comparison to the analysis by Chakrabarty \& Raychaudhury}

Chakrabarty \& Raychaudhury (2008) used the GC kinematic database presented in Paper I to study the dark matter content of NGC 4636 using the non-parametric inverse algorithm CHASSIS (Chakrabarty \& Saha 2001). Their main finding was that the dark halo required to explain the GC kinematics is very concentrated. While a high concentration parameter $c_{\text {vir }}>9$ as derived by Chakrabarty \& Raychaudhury is consistent with our isotropic Jeans models for the blue GCs (which allow for a wide range of concentration parameters), their estimate for the total mass exceeds ours: the circular velocity curve shown in their Fig. 6 (left panel) rises to about $450 \mathrm{~km} \mathrm{~s}^{-1}$ at $\sim 10 \mathrm{kpc}$ and then declines, reaching a value of $\sim 370 \mathrm{~km} \mathrm{~s}^{-1}$ at $40 \mathrm{kpc}$. Our mass models (shown in Fig. 19), however, translate to significantly lower values of $v_{c}$ with maximal values around $360 \mathrm{~km} \mathrm{~s}^{-1}$ (at $R \simeq 10 \mathrm{kpc}$ ) and $300 \lesssim v_{c} \lesssim 340 \mathrm{~km} \mathrm{~s}^{-1}$ at $40 \mathrm{kpc}$. Recently, their work has been complemented by an X-ray study which we discuss in the following section.

\subsection{Comparison to the analysis by Johnson et al.}

In their recent work on the X-ray halo of NGC 4636 Johnson et al. (2009) use a very detailed analysis of deep ( $80 \mathrm{ks}$ ) archival Chandra data to derive a mass profile which they compare to the dynamical modelling by Chakrabarty \& Raychaudhury (2008). Again, the concentration parameters derived for the NFW dark 


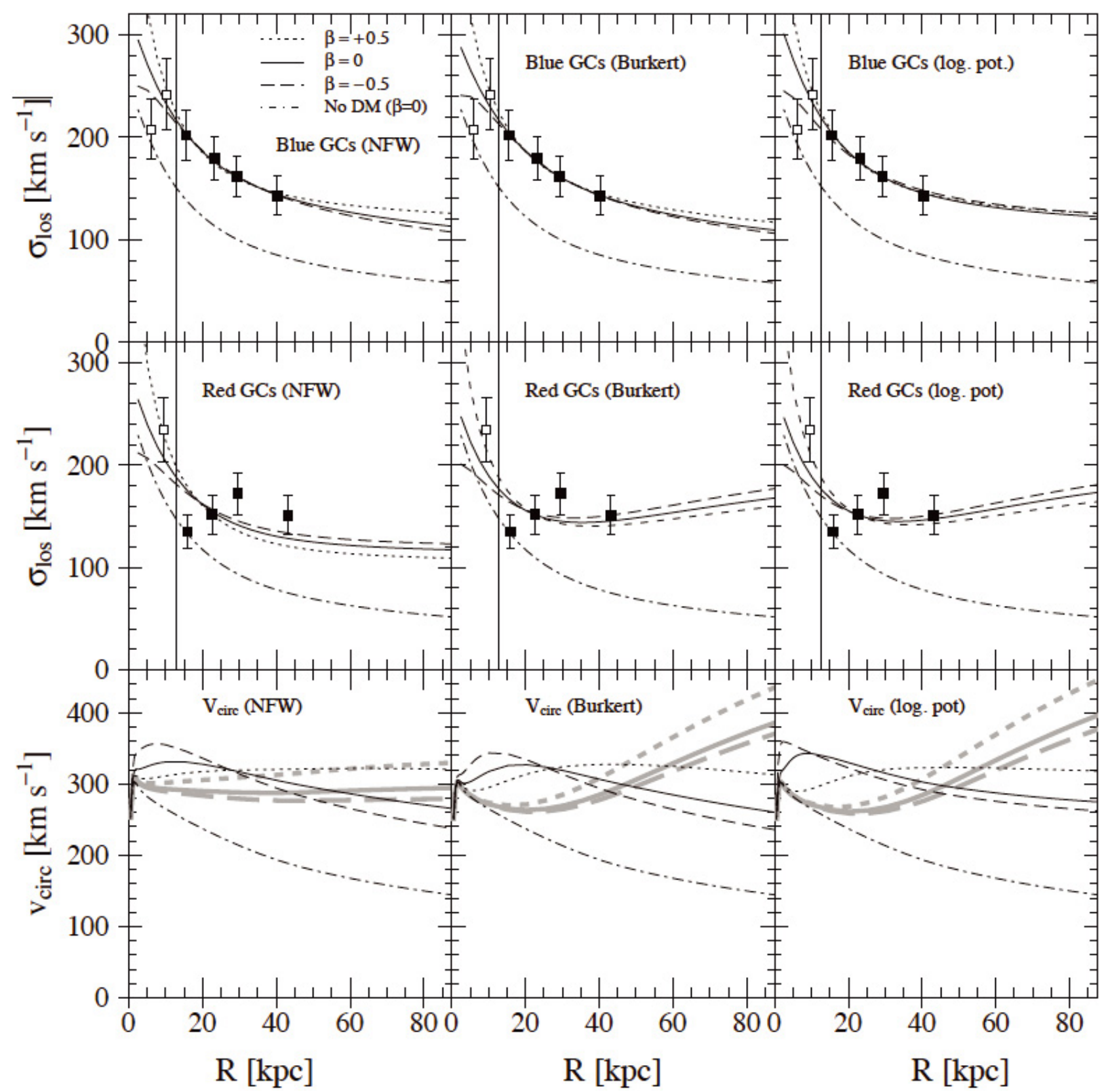

Fig. 19. Observed and modelled GC velocity dispersion profiles. Top row: models for the blue GCs (sample BlueFinal). From left to right, the panels show the best-fit models for an NFW halo, Burkert halo and the logarithmic potential. The solid lines are the isotropic models, dashed and short-dashed lines are the tangential $(\beta=-0.5)$ and radial $(\beta=+0.5)$ models, respectively. The dash-dotted line is the (isotropic) model without dark matter. The thin vertical line at $\simeq 13 \mathrm{kpc}$ indicates the radial range inside which blue and red GCs cannot be distinguished. The data points used in the modelling are shown as filled squares (see also Table 7). The model parameters are listed in Table 9. Middle row: the same for the red GCs (RedFinal). Bottom row: circular velocity curves for the best-fit models. Again, from left to right, the results for the NFW halo, Burkert halo and the logarithmic potential are shown. The line styles are the same as in the upper graphs, with thin black lines for the blue GCs while the respective models for the red GCs are shown as thick grey lines.

halo models are high, with values between 18 and 20. A key finding of their analysis is that the derived mass profile depends strongly on whether the metal abundance gradient of the X-ray halo is taken into account. While the overall shape of the mass profile remains the same, the inclusion of the abundance gradient reduces the mass at all radii by a factor of about 1.6 (see their Fig. 4). Moreover, both models show the same behaviour for large radii where the enclosed mass rises as $r^{1.2}$, a feature that was also found by Loewenstein \& Mushotzky (2003). To compare our dispersion measurements to the NFW profiles derived by Johnson et al. (2009), we proceed as follows: we calculate the velocity dispersion profiles expected for the blue GCs for the isotropic case $(\beta=0)$, adopting the NFW parameters given in their Sect. 4.2.

Johnson et al. parametrise their NFW halos in terms of concentration $c$ and the scale radius $r_{\mathrm{s}}$. Table 10 lists their values together with the corresponding density $\rho_{\mathrm{S}}$ and the virial parameters $^{6}$.

6 Note that Johnson et al. (2009) use a different definition of the virial parameters, i.e. $R_{200}=c \cdot r_{\mathrm{s}}$, where $R_{200}$ is the radius within which the mean density equals 200 times the critical density of the Universe. 
Y. Schuberth et al.: Dynamics of the NGC 4636 globular cluster system. II.
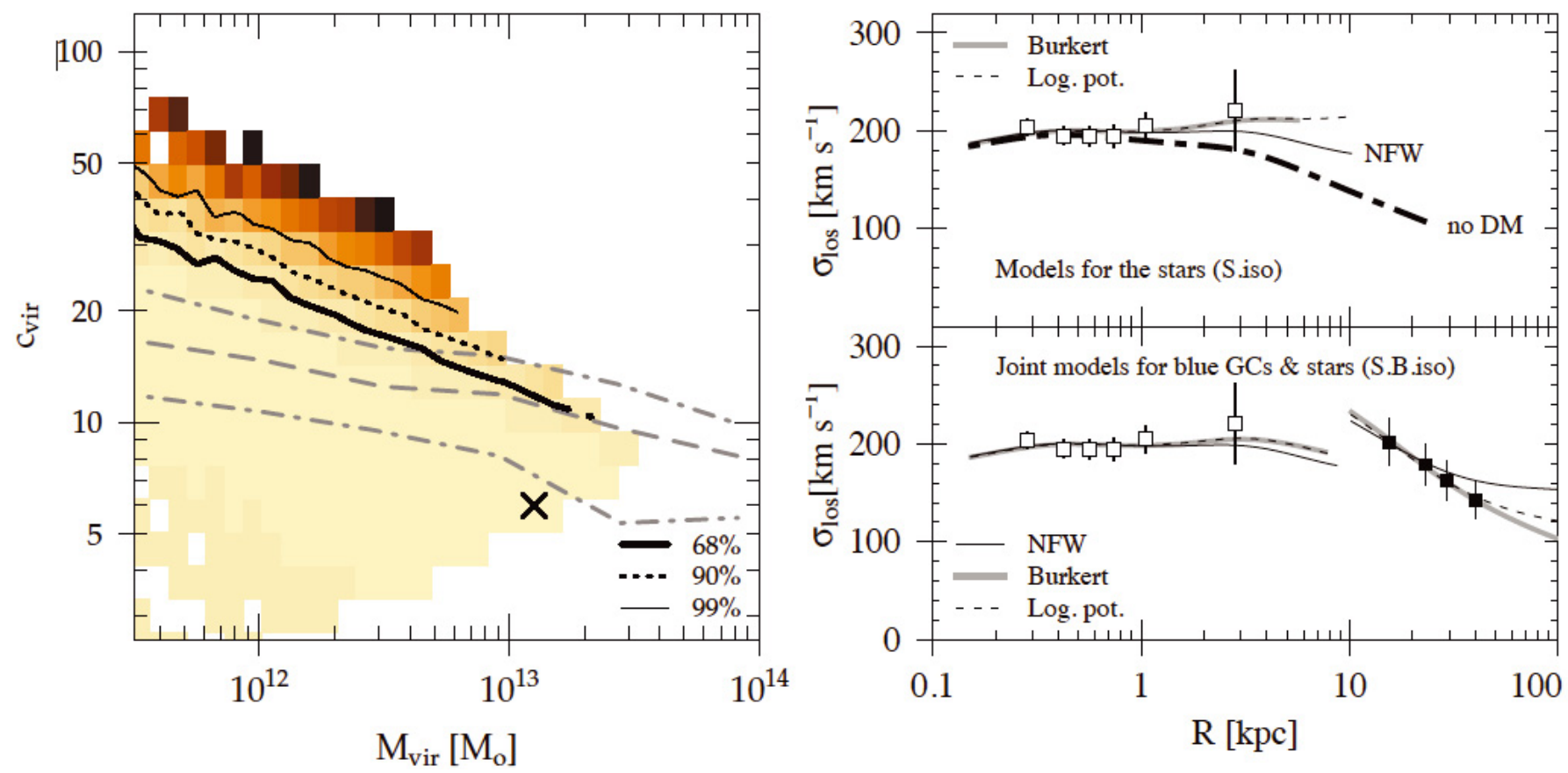

Fig. 20. Modelling the stellar velocity dispersion profile. Left: Jeans models for the stars (Bender et al. 1994 data) for an NFW-type dark halo and $\beta=0$. The parameters are shown in the $\left(M_{\mathrm{vir}}, c_{\mathrm{vir}}\right)$-plane. The thick solid, dashed and thin solid lines indicate the 68,90 , and 99 per cent confidence limits. The cross indicates the location of the minimum $\chi^{2}$ value. The model parameters are listed in Table 9. The long-dashed (dash-dotted) lines show the median (68 per cent values) for simulated NFW halos as found by Bullock et al. (2001). Right: modelled velocity dispersion profiles for the stars (Bender et al. 1994 data, shown as unfilled squares). The upper sub-panel shows the best-fit isotropic models for the stars. The thin solid line shows the model for an NFW-type dark halo, and the thin dashed line is the model for the logarithmic potential. The Burkert halo is shown as thick grey line. The thick dot-dashed line is the model without dark matter. The lower sub-panel shows the joint models for the blue GCs and the stellar velocity dispersion profile (models S.B.iso). The line-styles are the same as in the upper panel. The black squares show the velocity dispersion profile for the blue GCs (sample BlueFinal). The halo parameters are given in Table 9.

Table 10. Parameters of the NFW halos derived by Johnson et al. (2009).

\begin{tabular}{lccccc}
\hline \hline Model & $\begin{array}{c}r_{\mathrm{s}} \\
{[\mathrm{kpc}]}\end{array}$ & $c$ & $\begin{array}{c}R_{200} \\
{[\mathrm{kpc}]}\end{array}$ & $\begin{array}{c}\varrho_{\mathrm{s}} \\
{\left[M_{\odot} \mathrm{pc}^{-3}\right]}\end{array}$ & $\begin{array}{c}M_{200} \\
{\left[10^{12} M_{\odot}\right]} \\
(6)\end{array}$ \\
\hline $\mathrm{J} 1)$ & $(2)$ & $(3)$ & $(4)$ & $(5)$ & $(6)$ \\
$\mathrm{J} 2$ & $21.8 \pm 0.9$ & $20.1 \pm 0.8$ & $438 \pm 25$ & $0.0359 \pm 0.0036$ & $9.8_{-2.2}^{+2.6}$ \\
$\mathrm{~J} 3$ & $24.6 \pm 0.9$ & $18.0 \pm 0.6$ & $443 \pm 22$ & $0.0270 \pm 0.0023$ & $10.0_{-1.9}^{+2.4}$ \\
\hline
\end{tabular}

Notes. The first column labels the models in order of their appearance in Sect. 4.2 of Johnson et al. (2009), where J1: X-ray data, total mass; J2: X-ray data, stars subtracted; J3: X-ray profile including metal abundance gradient, stars subtracted. Columns 2 and 3 are the parameters quoted by these authors, Col. 4 gives $R_{200}$ in units of kpc. The corresponding values for the density $\varrho_{\mathrm{s}}$ and $M_{200}$, i.e. the enclosed mass at $R_{200}$ are given in Cols. 5 and 6.

For consistency, we adopt for these calculations a distance of $16 \mathrm{Mpc}$, i.e. the value used by Chakrabarty \& Raychaudhury and Johnson et al. Using the NFW parameters given in Table 10, we compute the expected velocity dispersion profiles.

These models for the blue GCs are compared to the observations in the upper panel of Fig. 22. Since Johnson et al. assumed a very low stellar mass-to light ratio, the difference between models $\mathrm{J} 1$ and $\mathrm{J} 2$, (i.e. the X-ray mass estimate without abundance gradient) before and after the subtraction of the stellar component is small. The corresponding velocity dispersions lie well above the data points.
A much better agreement between X-ray and GC based mass estimates is achieved when the metal abundance gradient of the $\mathrm{X}$-ray gas is taken into account. Model J3 agrees, within the uncertainties, with the GC data out to about $30 \mathrm{kpc}$. For the abundance gradient corrected mass profile shown in Fig. 4 of Johnson et al., one obtains a very similar velocity dispersion profile ${ }^{7}$. The $r^{1.2}$ rise for large radii, however, leads to an almost constant velocity dispersion profile for $R \gtrsim 40 \mathrm{kpc}$. For reference, we also plot, in Fig. 22, the best-fit isotropic NFW model derived for the blue GCs assuming a distance of $16 \mathrm{Mpc}$ and the adjusted $\Upsilon_{\star, R}=6.4$.

\subsection{Are all GCs bound to NGC 4636 ?}

Objects with velocities in excess of the escape velocity are probable interlopers. Due to the logarithmic divergence of the NFW potential, the escape velocity is not defined. But, in any spherical potential bound particles travel on planar orbits, and energy and angular momentum conservation are used to derive the following expression:

$v_{\mathrm{p}}^{2}=\frac{2 r_{\mathrm{a}}^{2}\left(\Phi\left(r_{\mathrm{p}}\right)-\Phi\left(r_{\mathrm{a}}\right)\right)}{r_{\mathrm{a}}^{2}-r_{\mathrm{p}}^{2}}$,

where $v_{\mathrm{p}}$ is the pericentric velocity, $r_{\mathrm{p}}$ and $r_{\mathrm{a}}$ are the pericentre and apocentre distances, respectively.

The gravitational potential $\Phi(r)$ given by

$\Phi(r)=-4 \pi\left[\frac{1}{r} \int_{0}^{r} \varrho(s) s^{2} \mathrm{~d} s+\int_{r}^{\infty} \varrho(s) s \mathrm{~d} s\right]$,

7 For this calculation we used a piecewise fit to the data in Fig. 4 of Johnson et al. (2009). 


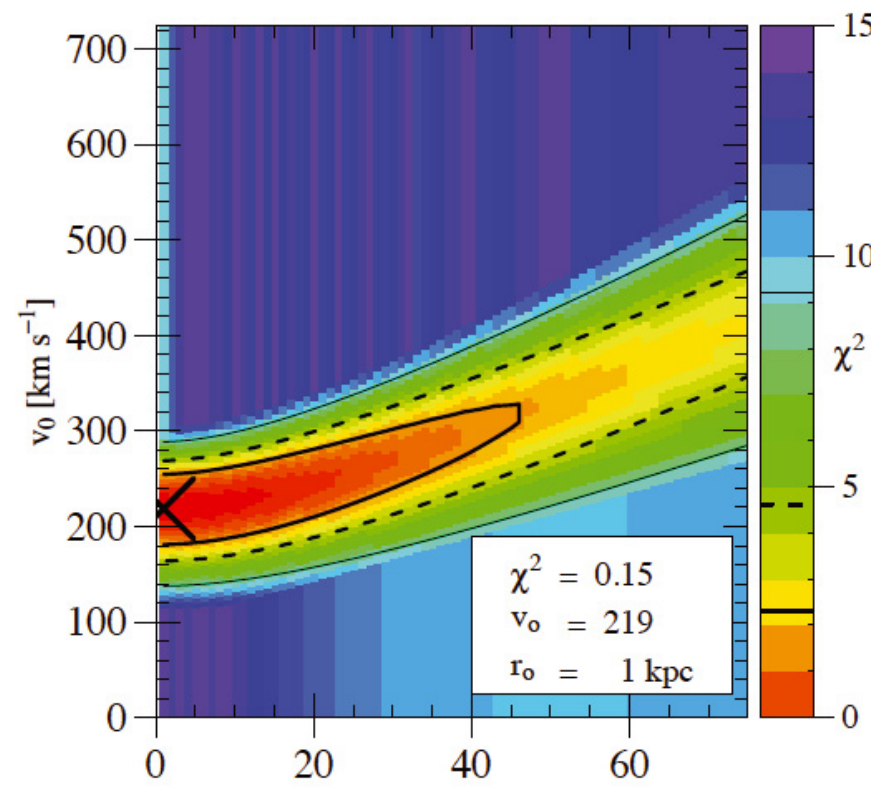

BLUE-B-0.5 $\quad \mathrm{r}_{0}[\mathrm{kpc}]$

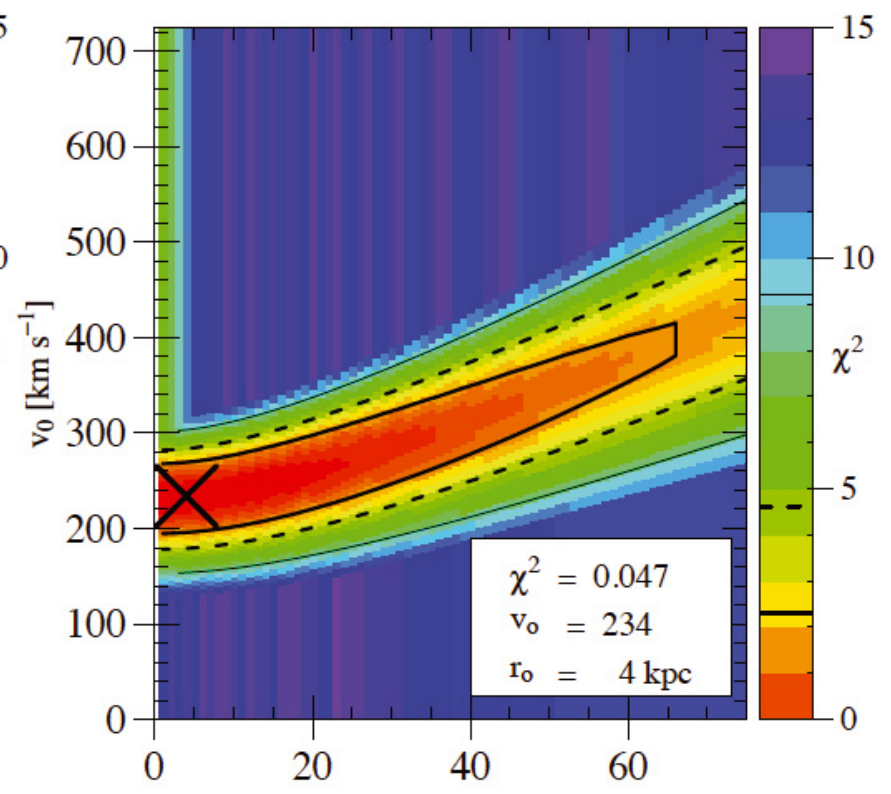

BLUE-ISO $\quad \mathrm{r}_{0}[\mathrm{kpc}]$

Fig. 21. NGC 4636 Jeans models the blue GCs (final sample) where the dark matter component is represented by a logarithmic potential Eq. (16). Left: models for a mild tangential bias with $\beta=-0.5$. Right: isotropic models $(\beta=0)$. In both panels, the best-fit solution is marked by a cross, and the thick solid, dashed and thin solid lines show the 68,90, and 99 per cent contour levels, respectively. The parameters of the halos shown here are also listed in Table 9.

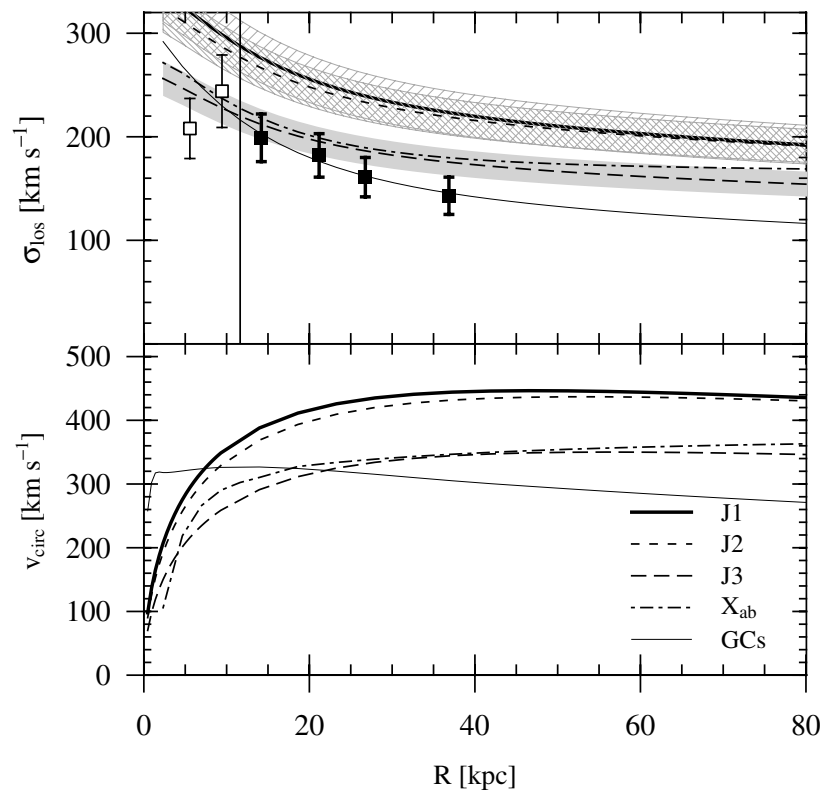

Fig. 22. Comparison to the NFW halos derived by Johnson et al. (2009) with the parameters listed in Table 10. Upper panel: velocity dispersion profiles. The data points show our final blue GC sample (same as in Fig. 19, left panel but for a distance of $16 \mathrm{Mpc}$ ). The thick solid line shows model J1; model J2 is shown as short-dashed line, the dashed regions show the corresponding uncertainties. The long-dashed line is model J3, and the grey area shows the uncertainties. The dash-dotted line (labelled $X_{\mathrm{ab}}$ ) corresponds to the mass profile (incorporating the abundance gradient) shown in Fig. 4 of Johnson et al. The thin solid line is the best-fit model for the GCs $\left(r_{\mathrm{s}}=20 \mathrm{kpc}, \varrho_{\mathrm{s}}=0.012, \Upsilon_{\star, R}=6.4\right)$. Lower panel: circular velocity curves. The line styles are the same as in the upper panel.

where $\varrho=\varrho_{\text {stars }}+\varrho_{\mathrm{DM}}$ is numerically integrated using the NGC 4636 stellar mass profile and the NFW halo dark matter density profile obtained from the blue GCs (model B.iso).
Objects outside a given curve have apocentric distances larger than the corresponding value of $r_{\mathrm{a}}$. The set of curves shown in Fig. 23 is obtained from Eq. (17) by fixing $r_{a} \in\{40,60,100,150,200,300\} \mathrm{kpc}$.

For the two blue GCs (objects 3.1:69 and 3.2:65) with good velocity measurements ( $v_{\text {helio }}=1428 \pm 37$ and $1441 \pm 28 \mathrm{~km} \mathrm{~s}^{-1}$, respectively) at a galactocentric distance of $\approx 34 \mathrm{kpc}$, we find apocentric distances of more than $150 \mathrm{kpc}$. Given that these conditions are extreme, an unknown population of GCs with large apogalactic distances may be present in the bulk of velocities. However, the question whether these GCs are bound or unbound, cannot be answered. Recall that, even in the Milky Way system, some GCs have Galactocentric distances of more than $100 \mathrm{kpc}$. How do these objects compare to the GCs with surprisingly high relative velocities in the NGC 1399 GCS identified by Richtler et al. (2004)? These authors show, in their Fig. 20, that the objects in their sample of (about a dozen) GCs with heliocentric velocities below $800 \mathrm{~km} \mathrm{~s}^{-1}$ (which corresponds to velocities of at least $640 \mathrm{~km} \mathrm{~s}^{-1}$ with respect to NGC 1399) have apogalactic distances between 100 to $200 \mathrm{kpc}$ (with one GC even featuring $r_{\mathrm{a}} \simeq 400 \mathrm{kpc}$ ). However, as shown in Schuberth et al. (2008), blue, velocity-confirmed NGC 1399 GCs are still found at distances of about $200 \mathrm{kpc}$. Thus, while the large number of GCs with apogalactic distances of $r_{\mathrm{a}} \approx 200 \mathrm{kpc}$ might be surprising, it is well possible that these objects belong to the very extended NGC 1399 GCS. Another scenario (cf. Schuberth et al. 2010) is that these metal-poor GCs were stripped from infalling galaxies which is not unlikely for a galaxy such as NGC 1399 which is the central galaxy in a relatively dense cluster. In the NGC 1399 GCS, the most extreme combination of radial distance and velocity is that of gc381.7 (from the catalogue of Bergond et al. 2007) which would have an apogalactic distance of the order 0.5 to $1 \mathrm{Mpc}$ (Schuberth et al. 2008), i.e. of at least twice the Fornax cluster core radius. 
Y. Schuberth et al.: Dynamics of the NGC 4636 globular cluster system. II.

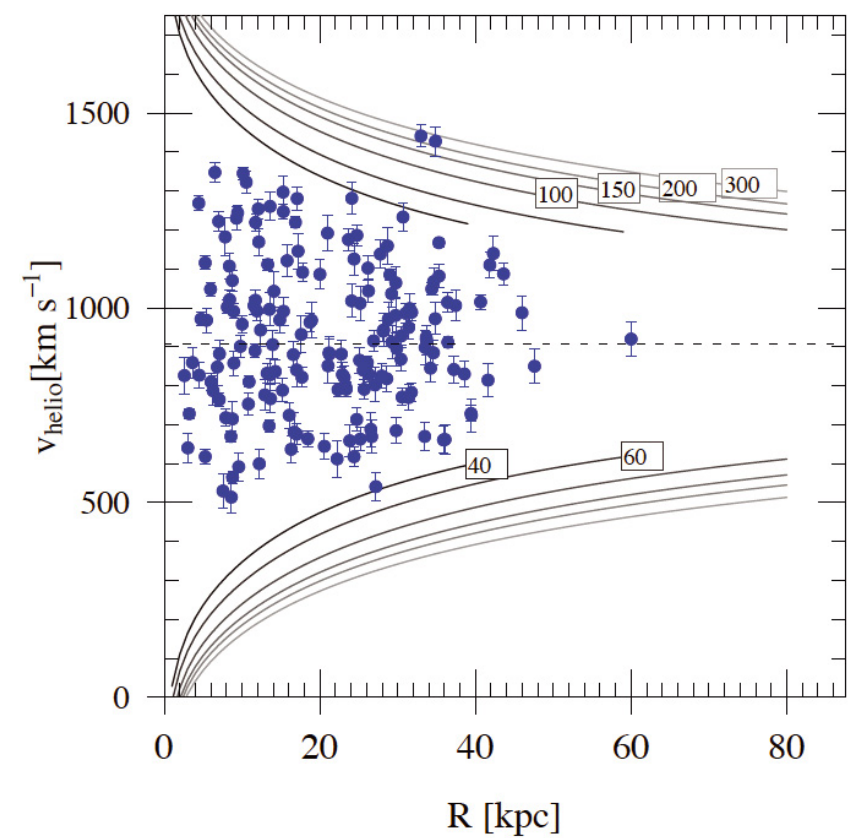

Fig. 23. Radial velocity versus galactocentric distance for blue GCs with velocity uncertainties $\Delta v<50 \mathrm{~km} \mathrm{~s}^{-1}$. The curves, calculated from Eq. (17) for the total mass as given in model B.iso (Table 9 NFW halo), indicate pericentric velocities for fixed apocentric distances. Objects outside a given line have apocentric distances of at least the value for which the curve was calculated.

In the case of NGC 4636, the two blue GCs with extreme velocities are remarkable. Although the estimated apogalactic distances are smaller than those of the extreme GCs near NGC 1399, one has to take into account that the NGC 4636 GCS appears to be truncated, and that almost no GCs are found beyond $\approx 60 \mathrm{kpc}$. Moreover, NGC 4636 is relatively isolated and does not show any signs of recent major mergers (Tal et al. 2009). This would make these objects candidates for a population of "vagrant" GCs belonging to the Virgo cluster rather than being genuine members of the NGC 4636 GCS.

\subsection{Stellar mass-to-light ratio}

Our value of the stellar $M / L_{R}$-value of 5.8, which we need under isotropy to model the central velocity dispersion data, is not directly supported by theoretical single stellar population synthesis models. Depending on the adopted stellar initial mass function (IMF), models predict values around 3.5-4 for metal-rich old populations (Bruzual \& Charlot 2003; Thomas et al. 2003; Maraston et al. 2003; Percival et al. 2009) and can reach up to 4.7 for super-solar abundance even for IMFs with a gentle slope in the mass-poor domain like the Kroupa (2001) IMF.

Of course, one could surely find an appropriate radial bias which at a given mass enhances the projected velocity dispersion in the central regions and thus would permit a lower $M / L$-value. This sort of fine-tuning is somewhat artificial and moreover is not supported by observational evidence. NGC 4636 exhibits in the analysis of Kronawitter et al. (2000) a tangential bias, though at larger radii, and is isotropic in its inner region.

A good reference to stellar $M / L$-values of the inner regions of elliptical galaxies is the study of Cappellari et al. (2006). These authors used the SAURON integral-field spectrograph to compare the $I$-band dynamical $M / L$ with the $\left(M / L_{\text {pop }}\right)$ obtained from stellar population models for a sample of 25 early-type (E/S0) galaxies.

For the 24 sample galaxies which lie in the $I$-band magnitude range $-20 \lesssim M_{I} \lesssim-24$ they find a correlation between the dynamical $M / L$ and the galaxy luminosity, in the sense that $M / L$ weakly increases with luminosity as $L_{I}^{0.32}$ (see. their Fig. 9, and Eq. (9) for the fit).

To address the question whether these observed $M / L_{\mathrm{dyn}}$ variations are due to a change in the stellar populations or to differences in the dark matter fraction, Cappellari et al. plot in their Fig. 17 the dynamical $M / L_{\text {dyn }}$ as a function of $M / L_{\text {pop }}$. Both quantities are correlated but while $M / L_{\text {dyn }} \geq M / L_{\text {pop }}$ for all sample galaxies ${ }^{8}$, the data points clearly lie off the one-to-one relation. The authors consider the lower luminosity fast rotators and the high luminosity slowly rotating galaxies separately (NGC 4636 would belong to the latter group). For old (age $>7$ Gyr) galaxies they find that the dark matter fraction within one effective radius increases from zero to about 30 per cent as the dynamical mass to light ratio increases from 3 to 6 . At a given $\left(M / L_{\text {pop }}\right)$, the massive slow rotators have higher dynamical $M / L$ values than the less massive fast rotators. How does NGC 4636 fit into this picture?

For NGC 4636 the Maraston et al. (2003) SSP model predicts an $M / L_{\mathrm{pop}, I}=3.27$ (for solar metallicity and an age of $13 \mathrm{Gyr}$ ). Converting this to the $B$ and $R$ bands, one obtains $M / L_{\mathrm{pop}, B}=6.6$ and $M / L_{\mathrm{pop}, R}=3.9$, respectively. Gerhard et al. quote an even lower value $M / L_{\mathrm{pop}, B}=5.9$.

What would we expect from the relations given by Cappellari et al.?

From Table 1, one obtains $M_{I, 4636}=-23.3$, and (for $M_{I, \odot}=4.08$, Lang 1999) from Eq. (9) in Cappellari et al., we thus would expect a dynamical $M / L_{I}=4.7$, corresponding to $M / L_{\mathrm{dyn}, R}=5.7$.

However, the models of Cappellari et al. (2006) have, by definition, a radially constant $M / L$, while our $M / L$ depends on radius and reaches $M / L_{\mathrm{dyn}, R} \approx 8$ at the effective radius.

Cappellari et al. speculate on the possibility that the difference between dynamical and population $M / L$ is due to a higher dark matter content of more luminous galaxies, but the general question is whether it is appropriate to apply SSP models to composite stellar systems. Let us consider $\omega$ Centauri, probably the dynamically best investigated stellar system, which is unrelaxed and composed of different populations. van de Ven et al. (2006) quote a $V$-band $M / L$ of $2.5 \pm 0.1$. The metallicity distribution of stars in $\omega$ Cen has a maximum at $[\mathrm{Fe} / \mathrm{H}] \approx-1.7$ with a broad tail towards higher metallicities (e.g. Hilker et al. 2004; Calamida et al. 2009). The more metal-rich populations are probably also younger by a few Gyr. From the population synthesis market, we cite Percival et al. (2009) who quote 2.3 as the value for a population with $[\mathrm{Fe} / \mathrm{H}]=-1.7 \mathrm{dex}$ and an age of $13.5 \mathrm{Gyr}$, and 2.0 for a population with $[\mathrm{Fe} / \mathrm{H}]=-1.3 \mathrm{dex}$ and an age of $10 \mathrm{Gyr}$, adopting a Kroupa IMF. Without aiming at precision, the composite "population" $M / L$ will probably not reach the dynamical value of 2.5 , unless there are old metal-rich populations, for which there is no evidence, so $\omega$ Centauri is at least a mild example without dark matter, where the dynamical mass is larger than the population mass.

However, an elliptical galaxy is a composite system with a long and complicated star formation history. If the IMF in a local

\footnotetext{
$8 M / L_{\text {pop }}$ was estimated using the Vazdekis et al. (1996); Vazdekis (1999) stellar population models assuming a Kroupa (2001) IMF, and the dynamical $M / L$ was obtained from Schwarzschild modelling of the SAURON data.
} 
star formation event is universal, there is no guarantee that the final mass function in a galaxy bears the same universality. Star formation occurs in star clusters and a galaxy's field population is composed of dissolved star clusters. If the mass spectrum of star clusters is a power-law like $m^{-2}$ then the dissolved population is the result of adding up many low-mass clusters, but fewer high-mass clusters, where the full stellar mass spectrum can be expected. Weidner \& Kroupa (2006) showed that, if the maximum stellar mass within a cluster depends on the clusters' mass, the resulting stellar mass function can be even steeper than a Salpeter mass function. There are no simulations of the final $M / L$ of an elliptical galaxy available, but since a Salpeterlike mass function increases the $M / L$ by factor of roughly 1.4 (e.g. Cappellari et al. 2006), it is plausible that there is not a strict universality of stellar mass functions among galaxies, but that the stellar mass function of an old elliptical galaxy may depend on the history of its assembly. In conclusion, a stellar $M / L_{R}$-ratio of 5.8 might well represent the stellar population.

Another consideration may be worthwhile: if we require the $M / L$-values to agree with the SSP predictions, we need a $M / L_{R}=4$ or smaller, lets say, 3.7. The dark halo, represented by a logarithmic halo, would assume parameters like $r_{0}=1 \mathrm{kpc}$ and $v_{0}=250 \mathrm{~km} \mathrm{~s}^{-1}$. The central density of dark matter then is $3.5 M_{\odot} / \mathrm{pc}^{3}$ under isotropy, and equality of stellar mass and dark mass is reached already at a radius of about $3.5 \mathrm{kpc}$. The central projected velocity dispersion is $170 \mathrm{~km} \mathrm{~s}^{-1}$ for the stellar mass alone and $192 \mathrm{~km} \mathrm{~s}^{-1}$ for the total mass. If that would be typical for elliptical galaxies (of which there is no evidence), scaling relations like the fundamental plane would dynamically be dominated by dark matter and the "conspiracy" between dark and luminous matter would reach a level even more difficult to understand than it is now.

Finding such a high central dark matter density prompts us to consider an older argument brought forward by Gerhard et al. (2001): the dark halos of elliptical galaxies in their sample turned out to exhibit a central density which is higher by a factor of at least 25 than those of spiral galaxies of similar luminosity, and also that the phase space densities are higher. Since in collisionless merging events phase space densities cannot grow, Gerhard et al. argued that it is unlikely that dark halos of ellipticals formed by the merging of dark halos of present-day spirals.

Gerhard et al.'s expression for the phase space density reads $f_{\mathrm{h}}=2^{3 / 2} \rho_{\mathrm{h}} / v_{\mathrm{h}}^{3}$, being $\rho_{\mathrm{h}}$ the central density and $v_{\mathrm{h}}^{3}$ the characteristic halo velocity of a logarithmic potential.

The above hypothetical dark matter density is about a factor 1000 higher than that of spirals (see Fig. 18 of Gerhard et al. 2001 ) and the phase space density of the corresponding halo is $6.7 \times 10^{-7}$ in units solar masses, pc, $\mathrm{km} \mathrm{s}^{-1}$, much higher than those of spirals. We conclude that with our example low $M / L$, it might not be possible to reach these densities and phase space densities by collionless accretion of spiral-like halos, and one has to resort to dark halos resembling those of dwarf spheroidals.

\subsection{MOND related issues}

The question whether elliptical galaxies fulfill the predictions of modified Newtonian dynamics (MOND, see e.g. Milgrom 2009; Sanders \& McGaugh 2002) obviously is a fundamental one. Ellipticals have so far been less in the focus of MOND than disk galaxies. The most compelling case of an apparently MONDian elliptical galaxy is the E4 galaxy NGC 2974 (Weijmans et al. 2008) where the extended HI disk permits a secure determination of the circular velocity which is constant out to $20 \mathrm{kpc}$.
In Paper I, we noted already that NGC 4636 seems to be consistent with being MONDian. Here, we plot, in the lower subpanel of Fig. 24 the MOND circular velocity curve (shown as thick solid line) for the stellar mass profile of NGC 4636 (for $M / L_{\star, R}=5.8$ ). The MOND circular velocity curve is obtained from the Newtonian one via the following equation:

$V_{\text {circ, } \mathrm{M}}^{2}=\frac{V_{\text {circ, } \mathrm{N}}^{2}}{2}+\sqrt{\frac{V_{\text {circ, } \mathrm{N}}^{4}}{4}+V_{\text {circ }, \mathrm{N}}^{2} \cdot a_{0} \cdot r}$

where $V_{\text {circ,N }}$ is the Newtonian velocity. For $a_{0}$ we adopt the value recommended by Famaey et al. (2007): $1.35 \times$ $10^{-8} \mathrm{~cm} \mathrm{~s}^{-2}$. Within the central $\approx 40 \mathrm{kpc}$, i.e. the radial range, for which we have data, the MOND circular velocity curve agrees fairly well with the best-fit joint model for stars and the blue GCs (models S.B.iso, Burkert halo, shown as thin dashed line).

Below we put NGC 4636 into the context of the more recent literature.

\subsubsection{Comparison to the $\mu_{\mathrm{OD}}$-relation by Donato et al.}

Recently Donato et al. (2009), in their extension of the work by Kormendy \& Freeman (2004), confirmed that the central surface density of galaxy dark matter halos is nearly constant and almost independent of galaxy luminosity. MOND-related aspects of this finding have been discussed by Gentile et al. (2009) and Milgrom (2009). Donato et al. assume that the dark matter halos of the galaxies are described by Burkert (1995) halos (cf. Eqs. (14) and (15)).

For the DM surface density $\mu_{0 D} \equiv r_{0} \varrho_{0}$, Donato et al. find the following relation to hold for galaxies in the magnitude range $-8 \geq M_{B} \geq-22$ :

$\log \left(\frac{\mu_{0 D}}{M_{\odot} \mathrm{pc}^{-2}}\right)=2.15 \pm 0.2$.

How does NGC 4636 fit into this picture? From the values given in Table 9, it appears that the dark matter density of our Burkert halo models (for the blue GCs and the stars) $(2.50 \lesssim$ $\left.\log \left(\mu_{0 D} / M_{\odot} \mathrm{pc}^{-2}\right) \lesssim 2.90\right)$ is too high with respect to the above value.

Going back to Fig. 18, however, one sees that for $r_{0} \gtrsim 20 \mathrm{kpc}$ the 68 per cent CL contour (thick solid line) of our isotropic models for the blue GCs lies within the range of values from Eq. (20) (shown as dot-dashed lines). In the upper panel of Fig. 24 we show, as an example, isotropic Jeans models for the blue GCs and the stellar component for $r_{0}=20 \mathrm{kpc}, \varrho_{0}=$ $1.115 \times 10^{-2}, \log \mu_{0 . D}=2.35$ (solid lines), i.e. a Burkert halo which is consistent (within the uncertainties) with the relation from Donato et al. (2009). For reference, the best-fit joint model is shown with dashed lines. The lower panel of Fig. 24 shows the corresponding circular velocity curves. Regarding the stars, we find $\chi^{2}$ values of $\chi_{\text {joint }}^{2}=2.0$ and $\chi_{\text {donato }}^{2}=2.9$ for the joint model and the model which is consistent with Eq. (20), respectively. For the blue GCs, the respective values are $\chi_{\text {joint }}^{2}<0.1$ and $\chi_{\text {donato }}^{2}=0.7$.

We conclude that the GC and stellar dynamics of NGC 4636 do not contradict the constant dark matter density relation, but we caution that the halos of ellipticals in the Donato et al. sample are constrained only by weak lensing shears, which do not probe the inner regions.

Gerhard et al., for their sample of ellipticals, implicitly found (multiplying their Eqs. (6) and (8)) $\rho_{0} \cdot r_{0}=635 L_{11}^{-0.1} h_{0.65}$, which for all practical purposes is constant (they used logarithmic halos 


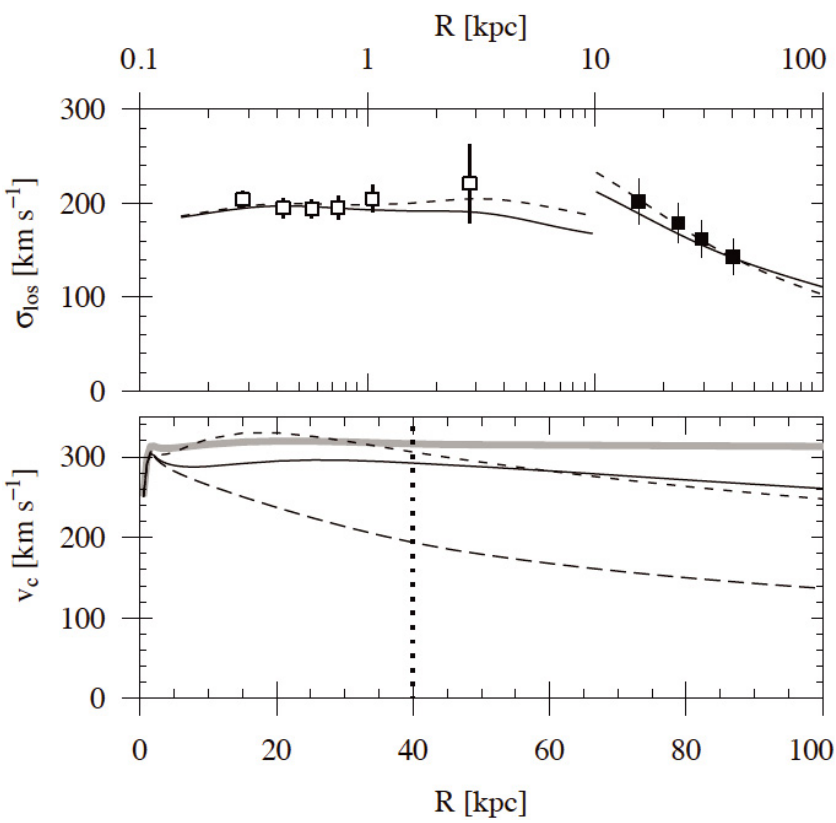

Fig. 24. Comparison to the results from Donato et al. (2009). Upper panel: open squares show the stellar velocity dispersion profile (BSG94), filles squares are the blue GCs (BlueFinal). The velocity dispersion profile for the joint isotropic model (blue GCs and stars, S.B.iso, Burkert halo) is shown as dashed line. The solid line shows the dispersion for a Burkert dark matter halo $r_{0}=20 \mathrm{kpc}$, $\varrho_{0}=1.115 \times 10^{-2}$ (i.e. $\log \mu_{0 . D}=2.35$ ) which is consistent with the relation by Donato et al. (2009) (see text for details). Lower panel: circular velocity curves. Again, the thin solid line shows the curve for the halo with $\log _{0 D}=2.35$, and the dashed line is the joint model. For reference, the circular velocity curve for the stars alone $\left(\Upsilon_{\star, R}=5.8\right)$ is shown as long-dashed line. The MOND circular velocity curve (Eq. (19)) is shown as a thick solid line. The dotted vertical line at $40 \mathrm{kpc}$ indicates the location of the outermost velocity dispersion bin for the blue GCs.

instead of Burkert halos). This fits better to our value and the question, whether disk galaxies and ellipticals really show the same surface density of dark matter, remains open.

\subsubsection{The baryonic Tully-Fisher relation and NGC 4636}

A severe irritation to the $\Lambda \mathrm{CDM}$ paradigm on galactic scales is the existence of a baryonic Tully-Fisher relation (BTFR) among spiral galaxies with an astonishingly small scatter, covering five orders of magnitude in mass, which reads (McGaugh 2005):

$M_{\mathrm{bar}}=50 \cdot v_{\mathrm{flat}}^{4}$,

where $v_{\text {flat }}$ is the circular velocity (in units of $\mathrm{km} \mathrm{s}^{-1}$ ) at a radius where the rotation curve becomes flat, and $M_{\mathrm{bar}}$ is the total mass in baryons (in units of $M_{\odot}$ ). Such a relation would naturally result from MOND (e.g. Milgrom 1983; Sanders \& McGaugh 2002). In the deep MOND regime:

$M=\frac{v_{c}^{4} \cdot a_{0}}{G}$,

$M$ being the total mass, $G$ the gravitational constant, and $a_{0}$ the MOND constant, which has the value $1.35_{-0.42}^{+0.28} \times 10^{-8} \mathrm{~cm} \mathrm{~s}^{-2}$ (Famaey et al. 2007). The factor of 50 in the above relation (Eq. (21)) corresponds to a somewhat higher value of $a_{0}=$ $1.5 \times 10^{-8} \mathrm{~cm} \mathrm{~s}^{-2}$ which still lies within the uncertainties of the value quoted above. The recent work of Stark et al. (2009) and Trachternach et al. (2009) confirmed this relation, which in Stark et al. formally reads $M_{\mathrm{bar}}=61 \cdot v_{\text {flat }}^{3.94}$ agreeing even better with the canonical value for $a_{0}$.

There also exists a BTFR for elliptical galaxies (Gerhard et al. 2001; Magorrian \& Ballantyne 2001; Thomas et al. 2007), but most elliptical galaxies lie off the spiral relation (Gerhard et al. 2001). Since the outermost radii in these dynamical studies may be still on the declining part of the circular velocity curves, it is interesting to put NGC 4636 into this picture. Although we cannot strictly distinguish between a constant circular velocity curve and a slightly declining one, the model with the flattest rotation curve has a circular velocity of $300 \mathrm{~km} \mathrm{~s}^{-1}$ and thus we expect a total baryonic mass of $4 \times 10^{11} M_{\odot}$. With the data from Table 1, we have $M_{R}=-22.6$, thus $5.5 \times 10^{10} L_{\odot}$ and a total baryonic mass of $3.2 \times 10^{11} M_{\odot}$, which would place NGC 4636 a bit below the relation for spirals. However, given the uncertainties in adopting distances, $M / L$-ratio and even the absolute solar $R$-magnitude, we are reluctant to assess these value as a clear displacement and repeat our conclusion from Paper I that NGC 4636 is consistent with the MONDian prediction.

In any way it is of fundamental interest to investigate more elliptical galaxies at large radii. If ellipticals and spirals would follow the same BTF-relation in spite of very different formation histories, the challenge for the cold dark matter paradigm of galaxy formation would be considerable.

\section{Conclusions}

We revisit the dynamics of the globular clusters system of NGC 4636 on the basis of 289 new globular cluster (GC) velocities. Including the data from Schuberth et al. (2006, Paper I), our total sample now consists of $460 \mathrm{GC}$ velocities, one of the largest sample obtained until now for a non-central elliptical galaxy. In addition we present new kinematical stellar data extending in radius the analysis by Kronawitter et al. (2000).

We model the total mass profile by the sum of the stellar mass plus a dark halo, for which we adopt different analytical forms. With our distance of $17.5 \mathrm{Mpc}$, we need a stellar $M / L_{R}$-value of 5.8 to satisfactorily reproduce the projected stellar velocity dispersion near the center under isotropy. This value is higher than values predicted from canonical population synthesis of an old, metal-rich population, resembling the results from the SAURON collaboration (Cappellari et al. 2006). We argue, however that the actual stellar mass function of an elliptical galaxy might be somewhat steeper than IMFs of local young star clusters.

We perform spherical Jeans-analyses independently for the red and the blue cluster population and fit dark matter profile parameters for NFW-profiles, for Burkert profiles and for logarithmic halos for different anisotropies. The fits using the red cluster populations are consistently worse than using the blue populations, a finding, which differs from our previous study of the central cluster galaxy NGC 1399 and for which we do not have a good explanation. Our recommended joint logarithmic halo which uses the stellar light and the blue GCs, has the parameters $r_{0}=8 \mathrm{kpc}$ and $v_{0}=237 \mathrm{~km} \mathrm{~s}^{-1}$.

The higher moments of the velocity distributions of the blue and red GC subpopulations are not really stable against the sample selections and thus do not permit to seriously constrain possible GC orbit anisotropies. However, they are consistent with the GC orbits being isotropic to a good approximation.

We compare our results with the mass profile, derived from X-ray analysis, of Johnson et al. (2009). When the element abundance gradient of the X-ray gas is not taken into account, 
the X-ray mass profile exceeds the GC mass profile by a significant factor. If the abundance gradient is accounted for, the agreement is good out to $30 \mathrm{kpc}$, but the X-ray mass profile still exceeds our mass profile beyond this radius. The might be a general problem of X-ray analyses, if strong abundance gradients are present, for example in galaxy clusters.

NGC 4636 almost falls onto the baryonic Tully-Fisher relation for spirals and behaves more or less MONDian, as already noted in Paper I. However, when modelled with a logarithmic halo, its halo surface density resembles that of other elliptical galaxies.

Acknowledgements. We thank an anonymous referee for constructive remarks and the editor for support. This research has made use of the NASA/IPAC Extragalactic Database (NED) which is operated by the Jet Propulsion Laboratory, California Institute of Technology, under contract with the National Aeronautics and Space Administration. T.R. acknowledges support from the Chilean Center of Astrophysics FONDAP No. 15010003 and from FONDECYT project No. 1100620 .

\section{References}

Annibali, F., Bressan, A., Rampazzo, R., Zeilinger, W. W., \& Danese, L. 2007, A\&A, 463, 455

Avni, Y. 1976, ApJ, 210, 642

Baldi, A., Forman, W., Jones, C., et al. 2009, ApJ, 707, 1034

Bender, R., Saglia, R. P., \& Gerhard, O. E. 1994, MNRAS, 269, 785 (BSG94)

Bergond, G., Athanassoula, E., Leon, S., et al. 2007, A\&A, 464, L21 (B+07)

Binggeli, B., Sandage, A., \& Tammann, G. A. 1985, AJ, 90, 1681

Binney, J. 1981, MNRAS, 196, 455

Binney, J., \& Tremaine, S. 1987, Galactic dynamics (Princeton, NJ: Princeton University Press), 747

Bruzual, G., \& Charlot, S. 2003, MNRAS, 344, 1000

Bullock, J. S., Kolatt, T. S., Sigad, Y., et al. 2001, MNRAS, 321, 559

Burkert, A. 1995, ApJ, 447, L25

Burstein, D., \& Heiles, C. 1982, AJ, 87, 1165

Côté, P., McLaughlin, D. E., Hanes, D. A., et al. 2001, ApJ, 559, 828

Calamida, A., Bono, G., Stetson, P. B., et al. 2009, ApJ, 706, 1277

Cappellari, M., \& Emsellem, E. 2004, PASP, 116, 138

Cappellari, M., Bacon, R., Bureau, M., et al. 2006, MNRAS, 366, 1126

Chakrabarty, D., \& Raychaudhury, S. 2008, AJ, 135, 2350

Chakrabarty, D., \& Saha, P. 2001, AJ, 122, 232

de Vaucouleurs, G., de Vaucouleurs, A., Corwin, H. G., et al. 1991, Third

Reference Catalogue of Bright Galaxies, Vol. 1-3, XII (Berlin Heidelberg

New York: Springer-Verlag)

Dirsch, B., Richtler, T., Geisler, D., et al. 2004, AJ, 127, 2114 (D+04)

Dirsch, B., Schuberth, Y., \& Richtler, T. 2005, A\&A, 433, 43

Donato, F., Gentile, G., Salucci, P., et al. 2009, MNRAS, 397, 1169

Evans, N. W., Wilkinson, M. I., Perrett, K. M., \& Bridges, T. J. 2003, ApJ, 583, 752

Famaey, B., Gentile, G., Bruneton, J.-P., \& Zhao, H. 2007, Phys. Rev. D, 75, 063002

Forman, W., Jones, C., \& Tucker, W. 1985, ApJ, 293, 102

Fraley, C., \& Raftery, A. E. 2002, J. Am. Stat. Assoc., 97, 611

Fraley, C., \& Raftery, A. E. 2006, MCLUST Version 3 for R: Normal

Mixture Modeling and Model-Based Clustering, University of Washington, Department of Statistics, Tech. Rep., 504

Gentile, G., Famaey, B., Zhao, H., \& Salucci, P. 2009, Nature, 461, 627

Gerhard, O., Kronawitter, A., Saglia, R. P., \& Bender, R. 2001, AJ, 121, 1936

Hilker, M., Kayser, A., Richtler, T., \& Willemsen, P. 2004, A\&A, 422, L9
Jensen, J. B., Tonry, J. L., Barris, B. J., et al. 2003, ApJ, 583, 712

Johnson, R., Chakrabarty, D., O'Sullivan, E., \& Raychaudhury, S. 2009, ApJ, 706,980

Jones, C., Forman, W., Vikhlinin, A., et al. 2002, ApJ, 567, L115

Kissler, M., Richtler, T., Held, E. V., et al. 1994, A\&A, 287, 463

Kormendy, J., \& Freeman, K. C. 2004, in Dark Matter in Galaxies, eds. S. Ryder, D. Pisano, M. Walker, \& K. Freeman, IAU Symp., 220, 377

Kronawitter, A., Saglia, R. P., Gerhard, O., \& Bender, R. 2000, A\&AS, 144, 53

Kroupa, P. 2001, MNRAS, 322, 231

Lang, K. R. 1999, Astrophysical formulae (Berlin: Springer-Verlag)

Lee, M. G., Park, H. S., Hwang, H. S., et al. 2010, ApJ, 709, 1083

Loewenstein, M., \& Mushotzky, R. 2003, Nucl. Phys. B Proc. Suppl., 124, 91

Loewenstein, M., Mushotzky, R. F., Angelini, L., Arnaud, K. A., \& Quataert, E. 2001, ApJ, 555, L21

Magorrian, J., \& Ballantyne, D. 2001, MNRAS, 322, 702

Mamon, G. A., \& Łokas, E. L. 2005, MNRAS, 363, 705

Maraston, C., Greggio, L., Renzini, A., et al. 2003, A\&A, 400, 823

Matsushita, K., Makishima, K., Ikebe, Y., et al. 1998, ApJ, 499, L13

McGaugh, S. S. 2005, ApJ, 632, 859

Merritt, D., \& Ferrarese, L. 2001, MNRAS, 320, L30

Milgrom, M. 1983, ApJ, 270, 365

Milgrom, M. 2009, MNRAS, 398, 1023

Navarro, J. F., Frenk, C. S., \& White, S. D. M. 1997, ApJ, 490, 493

Nilson, P. 1973, Nova Acta Regiae Soc. Sci. Upsaliensis Ser. V, 0

Park, H. S., Lee, M. G., Hwang, H. S., et al. 2010, ApJ, 709, 377

Percival, S. M., Salaris, M., Cassisi, S., \& Pietrinferni, A. 2009, ApJ, 690, 427

Proctor, R. N., \& Sansom, A. E. 2002, MNRAS, 333, 517

Prugniel, P., \& Heraudeau, P. 1998, A\&AS, 128, 299

Pryor, C., \& Meylan, G. 1993, in Structure and Dynamics of Globular Clusters, ASP Conf. Ser., 50, 357

Pu, S.-B., \& Han, Z.-W. 2011, Res. Astron. Astrophys., 11, 909

Richtler, T., Dirsch, B., Gebhardt, K., et al. 2004, AJ, 127, 2094

Richtler, T., Schuberth, Y., Hilker, M., et al. 2008, A\&A, 478, L23

Sanders, R. H., \& McGaugh, S. S. 2002, ARA\&A, 40, 263

Schlegel, D. J., Finkbeiner, D. P., \& Davis, M. 1998, ApJ, 500, 525

Schuberth, Y., Richtler, T., Dirsch, B., et al. 2006, A\&A, 459, 391

Schuberth, Y., Richtler, T., Bassino, L., \& Hilker, M. 2008, A\&A, 477, L9

Schuberth, Y., Richtler, T., Hilker, M., et al. 2010, A\&A, 513, A52

Schwarz, G. 1978, Ann. Stat., 6, 461

Stark, D. V., McGaugh, S. S., \& Swaters, R. A. 2009, AJ, 138, 392

Stephens, M. A. 1974, J. Am. Stat. Assoc., 69, 730

Stetson, P. B. 1987, PASP, 99, 191

Stetson, P. B. 1992, in Astronomical Data Analysis Software and Systems I, eds. D. M. Worrall, C. Biemesderfer, \& J. Barnes, ASP Conf. Ser., 25, 297

Strader, Romanowsky, A. J., Brodie, J. P., et al. 2011, ApJS, 197, 33

Tal, T., van Dokkum, P. G., Nelan, J., \& Bezanson, R. 2009, AJ, 138, 1417

Temi, P., Mathews, W. G., Brighenti, F., \& Bregman, J. D. 2003, ApJ, 585, L121

Temi, P., Brighenti, F., \& Mathews, W. G. 2007, ApJ, 666, 222

Thomas, D., Maraston, C., \& Bender, R. 2003, MNRAS, 339, 897

Thomas, J., Saglia, R. P., Bender, R., et al. 2007, MNRAS, 382, 657

Tonry, J., \& Davis, M. 1979, AJ, 84, 1511

Tonry, J. L., Dressler, A., Blakeslee, J. P., et al. 2001, ApJ, 546, 681

Trachternach, C., de Blok, W. J. G., McGaugh, S. S., van der Hulst, J. M., \& Dettmar, R.-J. 2009, A\&A, 505, 577

van de Ven, G., van den Bosch, R. C. E., Verolme, E. K., \& de Zeeuw, P. T. 2006, A\&A, 445, 513

van der Marel, R. P., \& Franx, M. 1993, ApJ, 407, 525

Vazdekis, A. 1999, ApJ, 513, 224

Vazdekis, A., Casuso, E., Peletier, R. F., \& Beckman, J. E. 1996, ApJS, 106, 307 Weidner, C., \& Kroupa, P. 2006, MNRAS, 365, 1333

Weijmans, A.-M., Krajnović, D., van de Ven, G., et al. 2008, MNRAS, 383, 1343

Woodley, K. A., Gómez, M., Harris, W. E., Geisler, D., \& Harris, G. L. H. 2010, AJ, 139, 1871 
Y. Schuberth et al.: Dynamics of the NGC 4636 globular cluster system. II.

\section{Appendix A: The velocity dispersion profile of NGC 4636}

Table A.1. NGC 4636 velocity dispersion profile from FORS 2 data.

\begin{tabular}{lcc}
\hline \hline Slit ID & $R$ & $\sigma$ \\
\hline & {$\left[{ }^{\prime \prime}\right]$} & {$\left[\mathrm{km} \mathrm{s}^{-1}\right]$} \\
104 & 67.9 & $185 \pm 24$ \\
103 & 64.7 & $142 \pm 25$ \\
100 & 54.8 & $147 \pm 22$ \\
99 & 51.3 & $186 \pm 18$ \\
98 & 47.9 & $197 \pm 20$ \\
97 & 44.7 & $213 \pm 17$ \\
96 & 41.2 & $178 \pm 13$ \\
95 & 37.6 & $199 \pm 11$ \\
94 & 34.1 & $179 \pm 14$ \\
89 & 13.7 & $197 \pm 10$ \\
86 & 4.8 & $201 \pm 7$ \\
85 & 0.7 & $233 \pm 7$ \\
84 & -2.5 & $199 \pm 7$ \\
83 & -5.5 & $192 \pm 8$ \\
82 & -8.7 & $186 \pm 8$ \\
81 & -12.2 & $199 \pm 9$ \\
80 & -15.3 & $196 \pm 9$ \\
79 & -18.2 & $201 \pm 9$ \\
78 & -21.1 & $202 \pm 10$ \\
75 & -30.8 & $212 \pm 10$ \\
74 & -33.7 & $192 \pm 16$ \\
73 & -36.5 & $198 \pm 16$ \\
70 & -47.5 & $182 \pm 13$ \\
69 & -51.0 & $207 \pm 23$ \\
68 & -54.5 & $202 \pm 20$ \\
67 & -57.9 & $171 \pm 20$ \\
66 & -61.1 & $207 \pm 21$ \\
65 & -64.6 & $178 \pm 18$ \\
64 & -67.4 & $145 \pm 23$ \\
\hline & & \\
& &
\end{tabular}

Notes. Column (1) gives the slit number on the FORS 2 Mask 1_1 from Paper I. The galactocentric distance $R$ (in units of arcseconds) is given in the second column. Positive and negative values of $R$ refer to positions to the north and south of the centre of NGC 4636 respectively. Column (3) lists the velocity dispersion (in $\mathrm{km} \mathrm{s}^{-1}$ ).

\section{Appendix B: The stellar mass profile of NGC 4636}

Here we give the piecewise approximation of the stellar mass profile of NGC 4636 (see Sect. 10.2) used in our modelling. In the following expressions, $x$ is in units of parsec, $D$ is the distance of NGC 4636 in Mpc (assumed to be $D=17.5$ in our modelling), and $\Upsilon_{\star, R}(=5.8)$ is the $R$-band stellar mass-to-light ratio. For $0<x \leq 3.0 \cdot D$ :

$p_{o}=\Upsilon_{\star, R}\left(\frac{D}{15}\right)^{2} \cdot 5.74 \times 10^{6}$,

for $3.0 \cdot D<x \leq 29.184 \cdot D$ :

$p_{1}(x)=\Upsilon_{\star, R}\left(\frac{D}{15}\right)^{2} \cdot \sum_{i=0}^{4} a_{i}\left(\frac{x}{D}\right)^{i}$,

where $a_{0}=1.322 \times 10^{7}, a_{1}=-1.118 \times 10^{7}, a_{2}=3.158 \times 10^{6}$, $a_{3}=-9.127 \times 10^{4}$, and $a_{4}=1.164 \times 10^{3}$.

For $29.184 \cdot D<x \leq 104.49 \cdot D$ :

$p_{2}(x)=\Upsilon_{\star, R}\left(\frac{D}{15}\right)^{2} \cdot \sum_{i=1}^{4} b_{i}\left(\frac{x}{D}\right)^{i}$

where $b_{1}=9.616 \times 10^{6}, b_{2}=1.034 \times 10^{6}, b_{3}=-9.133 \times 10^{3}$, and $b_{4}=25.78$

For $104.49 \cdot D<x \leq 2597.9 \cdot D$ :

$p_{3}(x)=\Upsilon_{\star, R}\left(\frac{D}{15}\right)^{2} \cdot \sum_{i=0}^{3} c_{i}\left(\frac{x}{D}\right)^{i}$,

where $c_{0}=6.101 \times 10^{8}, c_{1}=4.31 \times 10^{7}, c_{2}-1.507 \times 10^{4}$, and $c_{3}=1.996$

For $x>2597.9 \cdot D$

$A(x)=d_{1} \cdot \arctan \left(\left(\frac{x / D}{d_{0}}\right)+d_{2} \cdot\left(\frac{x / D}{d_{0}}\right)^{2}+d_{3} \cdot\left(\frac{x / D}{d_{0}}\right)^{3}\right)$

where $d_{0}=1.194 \times 10^{3}, d_{1}=4.221 \times 10^{10}, d 2=-0.0668$, and $d 3=0.003774$. 
Table C.1. Catalogue of globular cluster velocities.

\begin{tabular}{|c|c|c|c|c|c|c|c|c|c|c|c|}
\hline (1) & $\begin{array}{c}\text { RA } \\
\text { (J2000) } \\
(2)\end{array}$ & $\begin{array}{c}\text { Dec } \\
(\mathrm{J} 2000) \\
(3)\end{array}$ & $\begin{array}{c}R_{4636} \\
{[\operatorname{arcmin}]} \\
(4)\end{array}$ & $\begin{array}{c}\text { PA } \\
{[\mathrm{deg}]} \\
(5)\end{array}$ & $\begin{array}{c}m_{R} \\
{[\mathrm{mag}]} \\
(6)\end{array}$ & $\begin{array}{c}C-R \\
{[\mathrm{mag}]} \\
(7)\end{array}$ & $\begin{array}{c}v_{\text {spec }} \\
{\left[\mathrm{km} \mathrm{s}^{-1}\right]} \\
(8)\end{array}$ & $\begin{array}{c}v_{\text {final }} \\
{\left[\mathrm{km} \mathrm{s}^{-1}\right]} \\
(9)\end{array}$ & Sample & Cross-Identification(s) & (12) \\
\hline f01-03 & $12: 43: 04.83$ & $+02: 33: 06.8$ & 8.97 & 155.4 & 22.01 & 1.22 & $584 \pm 66$ & $584 \pm 66$ & Blue & & 1 \\
\hline f01-07 & $12: 42: 58.79$ & $+02: 33: 30.4$ & 8.07 & 164.0 & 22.20 & 1.77 & $871 \pm 34$ & $899 \pm 53$ & RedFinal & f06-15 & 1 \\
\hline f01-09 & $12: 43: 02.89$ & $+02: 33: 46.0$ & 8.17 & 156.6 & 21.14 & 1.08 & $814 \pm 43$ & $814 \pm 43$ & BlueFinal & & 1 \\
\hline f01-11 & $12: 43: 07.49$ & $+02: 34: 04.5$ & 8.43 & 148.5 & 21.55 & 1.75 & $573 \pm 18$ & $573 \pm 18$ & RedFinal & & 1 \\
\hline f01-14 & $12: 43: 02.07$ & $+02: 34: 33.2$ & 7.37 & 155.6 & 21.88 & 1.17 & $847 \pm 40$ & $859 \pm 74$ & Blue & f06-25 & 1 \\
\hline f01-15 & $12: 43: 03.79$ & $+02: 34: 43.3$ & 7.41 & 152.0 & 21.26 & 1.79 & $840 \pm 21$ & $840 \pm 21$ & RedFinal & & 1 \\
\hline f01-21 & $12: 43: 06.53$ & $+02: 35: 26.3$ & 7.16 & 144.5 & 20.63 & 1.37 & $912 \pm 15$ & $912 \pm 15$ & BlueFinal & & 1 \\
\hline f01-24 & $12: 43: 03.56$ & $+02: 36: 02.6$ & 6.24 & 146.8 & 22.14 & 1.89 & $995 \pm 36$ & $995 \pm 36$ & RedFinal & & 1 \\
\hline f01-25 & $12: 43: 02.62$ & $+02: 36: 08.9$ & 6.03 & 148.1 & 21.56 & 1.29 & $1233 \pm 35$ & $1233 \pm 35$ & BlueFinal & & 1 \\
\hline f01-26 & $12: 43: 09.35$ & $+02: 36: 19.1$ & 6.94 & 135.5 & 21.84 & 1.20 & $808 \pm 85$ & $808 \pm 85$ & Blue & & 1 \\
\hline f01-28 & $12: 43: 09.99$ & $+02: 36: 33.6$ & 6.88 & 133.1 & 22.67 & 1.59 & $1057 \pm 56$ & $1057 \pm 56$ & RedFinal & & 1 \\
\hline f01-30 & $12: 43: 10.42$ & $+02: 36: 48.6$ & 6.80 & 131.0 & 21.89 & 1.42 & $885 \pm 31$ & $885 \pm 31$ & BlueFinal & & 1 \\
\hline f01-31 & $12: 43: 00.89$ & $+02: 36: 53.5$ & 5.17 & 147.8 & 20.64 & 1.35 & $829 \pm 21$ & $829 \pm 21$ & BlueFinal & & 1 \\
\hline f01-32 & $12: 43: 04.39$ & $+02: 37: 00.8$ & 5.59 & 139.6 & 22.13 & 1.34 & $1460 \pm 52$ & $1460 \pm 52$ & Blue & & 1 \\
\hline f01-33 & $12: 43: 05.20$ & $+02: 37: 06.2$ & 5.66 & 137.4 & 22.46 & 1.54 & $971 \pm 42$ & $971 \pm 42$ & BlueFinal & & 1 \\
\hline f01-34 & $12: 43: 04.32$ & $+02: 37: 15.9$ & 5.39 & 138.0 & 22.39 & 1.71 & $1117 \pm 49$ & $1117 \pm 49$ & RedFinal & & 1 \\
\hline f01-35 & $12: 42: 57.82$ & $+02: 37: 20.1$ & 4.40 & 153.2 & 21.98 & 1.48 & $521 \pm 63$ & $525 \pm 101$ & Blue & f02-01 & 1 \\
\hline f01-36 & $12: 42: 59.42$ & $+02: 37: 27.1$ & 4.50 & 148.0 & 21.34 & 1.78 & $897 \pm 26$ & $868 \pm 36$ & RedFinal & f02-02 & 1 \\
\hline f01-37 & $12: 43: 04.80$ & $+02: 37: 31.8$ & 5.28 & 135.1 & 22.24 & 1.61 & $930 \pm 47$ & $930 \pm 47$ & RedFinal & & 1 \\
\hline f01-38 & $12: 43: 03.71$ & $+02: 37: 37.2$ & 5.02 & 136.5 & 20.18 & 1.54 & $845 \pm 28$ & $840 \pm 35$ & BlueFinal & f02-04, f06-55 & 1 \\
\hline f01-41 & $12: 43: 06.18$ & $+02: 37: 56.5$ & 5.26 & 129.2 & & & $572 \pm 94$ & $612 \pm 109$ & NoPhot & f02-07 & 1 \\
\hline f01-42 & 12:43:03.61 & $+02: 38: 00.4$ & 4.73 & 133.5 & 20.66 & 1.54 & $1043 \pm 33$ & $1018 \pm 42$ & BlueFinal & f02-08 & 1 \\
\hline f01-43 & 12:43:10.67 & $+02: 38: 11.1$ & 6.04 & 120.7 & 21.86 & 1.75 & $780 \pm 28$ & $754 \pm 36$ & RedFinal & f12-11 & 1 \\
\hline f01-44 & $12: 43: 02.43$ & $+02: 38: 17.2$ & 4.33 & 133.5 & 22.05 & 1.80 & $1063 \pm 31$ & $997 \pm 49$ & RedFinal & f02-11 & 1 \\
\hline f01-45 & 12:43:06.38 & $+02: 38: 22.5$ & 5.04 & 125.0 & 22.10 & 1.11 & $754 \pm 58$ & $789 \pm 82$ & Blue & f12-12 & 1 \\
\hline f01-46 & $12: 42: 58.74$ & $+02: 38: 31.9$ & 3.52 & 141.0 & 20.01 & 1.60 & $830 \pm 13$ & $830 \pm 13$ & RedFinal & & 1 \\
\hline f01-48 & $12: 43: 03.04$ & $+02: 38: 45.5$ & 4.14 & 127.3 & 21.32 & 1.37 & $856 \pm 42$ & $851 \pm 45$ & BlueFinal & f02-18 & 1 \\
\hline f01-49 & $12: 42: 58.77$ & $+02: 38: 50.7$ & 3.29 & 137.5 & 22.04 & 1.05 & $1113 \pm 50$ & $1038 \pm 107$ & Blue & f02-19 & 1 \\
\hline f01-50 & $12: 42: 57.51$ & $+02: 38: 56.5$ & 3.01 & 140.6 & 21.70 & 1.31 & $1247 \pm 20$ & $1247 \pm 20$ & BlueFinal & & 1 \\
\hline f01-51 & $12: 42: 58.89$ & $+02: 39: 03.4$ & 3.16 & 134.5 & 21.27 & 1.05 & $724 \pm 33$ & $724 \pm 33$ & BlueFinal & & 1 \\
\hline fo1-53 & 12:42:58.92 & $+02: 39: 17.9$ & 3.00 & 131.1 & 21.75 & 1.50 & $927 \pm 27$ & $900 \pm 55$ & BlueFinal & f02-23 & 1 \\
\hline f01-54 & $12: 43: 01.98$ & $+02: 39: 25.3$ & 3.54 & 121.4 & 22.28 & 1.29 & $980 \pm 41$ & $917 \pm 87$ & Blue & f02-24 & 1 \\
\hline f01-55 & $12: 42: 58.65$ & $+02: 39: 35.2$ & 2.76 & 127.5 & 21.78 & 1.67 & $859 \pm 26$ & $826 \pm 44$ & RedFinal & f02-25 & 1 \\
\hline f01-56 & $12: 42: 58.64$ & $+02: 39: 43.0$ & 2.68 & 125.3 & 21.95 & 1.49 & $927 \pm 30$ & $829 \pm 42$ & BlueFinal & f02-26 & 1 \\
\hline f01-57 & $12: 42: 59.02$ & $+02: 39: 49.6$ & 2.70 & 122.2 & 21.11 & 1.74 & $1007 \pm 22$ & $964 \pm 42$ & RedFinal & f02-27 & 1 \\
\hline f02-01 & $12: 42: 57.82$ & $+02: 37: 20.1$ & 4.40 & 153.2 & 21.98 & 1.48 & $529 \pm 79$ & $525 \pm 101$ & Blue & f01-35 & 0 \\
\hline f02-02 & $12: 42: 59.42$ & $+02: 37: 27.1$ & 4.50 & 148.0 & 21.34 & 1.78 & $838 \pm 25$ & $868 \pm 36$ & RedFinal & f01-36 & 0 \\
\hline f02-04 & $12: 43: 03.71$ & $+02: 37: 37.2$ & 5.02 & 136.5 & 20.18 & 1.54 & $787 \pm 13$ & $840 \pm 35$ & BlueFinal & f01-38, f06-55 & 0 \\
\hline f02-05 & $12: 42: 57.31$ & $+02: 37: 43.3$ & 4.00 & 152.3 & 22.45 & 2.14 & $591 \pm 79$ & $591 \pm 79$ & RedFinal & & 1 \\
\hline f02-07 & $12: 43: 06.18$ & $+02: 37: 56.5$ & 5.26 & 129.2 & $\cdots$ & $\ldots$ & $652 \pm 56$ & $612 \pm 109$ & NoPhot & f01-41 & 0 \\
\hline f02-08 & $12: 43: 03.61$ & $+02: 38: 00.4$ & 4.73 & 133.5 & 20.66 & 1.54 & $992 \pm 27$ & $1018 \pm 42$ & BlueFinal & f01-42 & 0 \\
\hline f02-09 & $12: 42: 56.55$ & $+02: 38: 04.9$ & 3.60 & 152.4 & 20.57 & 1.12 & $867 \pm 29$ & $881 \pm 54$ & BlueFinal & $1.1: 34$ & 1 \\
\hline f02-10 & $12: 42: 56.18$ & $+02: 38: 11.2$ & 3.46 & 152.9 & 21.01 & 1.22 & $844 \pm 27$ & $932 \pm 47$ & BlueFinal & f06-61 & 1 \\
\hline f02-11 & $12: 43: 02.43$ & $+02: 38: 17.2$ & 4.33 & 133.5 & 22.05 & 1.80 & $931 \pm 38$ & $997 \pm 49$ & RedFinal & f01-44 & 0 \\
\hline f02-13 & $12: 42: 57.50$ & $+02: 38: 28.5$ & 3.38 & 145.7 & 21.59 & 1.07 & $1145 \pm 46$ & $1145 \pm 46$ & BlueFinal & & 1 \\
\hline f02-14 & $12: 42: 57.50$ & $+02: 38: 30.2$ & 3.36 & 145.4 & 19.83 & 1.27 & $1225 \pm 20$ & $1280 \pm 30$ & BlueFinal & f06-65 & 1 \\
\hline f02-15 & $12: 43: 04.45$ & $+02: 38: 35.8$ & 4.52 & 126.3 & 21.52 & 1.66 & $663 \pm 19$ & $663 \pm 19$ & RedFinal & & 1 \\
\hline f02-16 & $12: 43: 04.45$ & $+02: 38: 37.3$ & 4.50 & 126.0 & 21.19 & 1.42 & $829 \pm 30$ & $829 \pm 30$ & BlueFinal & & 1 \\
\hline f02-17 & $12: 43: 04.33$ & $+02: 38: 39.5$ & 4.45 & 125.8 & 21.30 & 1.61 & $724 \pm 21$ & $724 \pm 21$ & RedFinal & & 1 \\
\hline f02-18 & $12: 43: 03.04$ & $+02: 38: 45.5$ & 4.14 & 127.3 & 21.32 & 1.37 & $847 \pm 15$ & $851 \pm 45$ & BlueFinal & f01-48 & 0 \\
\hline f02-19 & $12: 42: 58.77$ & $+02: 38: 50.7$ & 3.29 & 137.5 & 22.04 & 1.05 & $964 \pm 95$ & $1038 \pm 107$ & Blue & f01-49 & 0 \\
\hline f02-21 & 12:43:01.05 & $+02: 39: 04.7$ & 3.55 & 128.1 & 21.97 & 1.40 & $729 \pm 80$ & $729 \pm 80$ & Blue & & 1 \\
\hline
\end{tabular}

Notes. Column (1) gives the spectrum identifier (mask:slit). Note that the new data have the prefix "f". For the purpose of cross-referencing, the data from Paper I are also included in this table. Columns (2) and (3) are the right ascension and declination, respectively (for objects with Washington photometry, the coordinates are those from Dirsch et al. 2005; for objects not in the photometric catalogue, the values were estimated from the pre-images). The projected distance from the centre of NGC 4636 (in units of arcminutes) is given in Col. (4). Column (5) is the position angle, (measured North over East). Columns (6) and (7) are the magnitude and colour as given by Dirsch et al. (2005). For the new objects, Col. (8) gives the heliocentric velocity measured for a given spectrum, for the data from Paper I, the final velocity given there is quoted. Column (9) gives the final velocity used in this study, for objects for which more than one spectrum was obtained, the error-weighted mean is given. Column (10) indicates the subsample ("R.25" denotes GCs with projected distances $R \leq 2$ '.5). Column (11) gives the cross-identifications within this list. The entry in Col. (12) is " 1 " for the first appearance of an object in this table. All subsequent entries for the same object are labelled " 0 ". 
Y. Schuberth et al.: Dynamics of the NGC 4636 globular cluster system. II.

Table C.1. continued.

\begin{tabular}{|c|c|c|c|c|c|c|c|c|c|c|c|}
\hline ID & $\begin{array}{c}\text { RA } \\
(\mathrm{J} 2000)\end{array}$ & $\begin{array}{c}\text { Dec } \\
(\mathrm{J} 2000)\end{array}$ & $\begin{array}{c}R_{4636} \\
\text { [arcmin] }\end{array}$ & $\begin{array}{c}\text { PA } \\
{[\mathrm{deg}]}\end{array}$ & $\begin{array}{c}m_{R} \\
{[\mathrm{mag}]}\end{array}$ & $\begin{array}{l}C-R \\
{[\mathrm{mag}]}\end{array}$ & $\begin{array}{c}v_{\mathrm{spec}} \\
{\left[\mathrm{km} \mathrm{s}^{-1}\right]}\end{array}$ & $\begin{array}{c}v_{\text {final }} \\
{\left[\mathrm{km} \mathrm{s}^{-1}\right]}\end{array}$ & Sample & Cross-Identification(s) & $1 \mathrm{st}$ \\
\hline f02-23 & $12: 42: 58.92$ & $+02: 39: 17.9$ & 3.00 & 131.1 & 21.75 & 1.50 & $874 \pm 47$ & $900 \pm 55$ & BlueFinal & f01-53 & 0 \\
\hline f02-24 & 12:43:01.98 & $+02: 39: 25.3$ & 3.54 & 121.4 & 22.28 & 1.29 & $855 \pm 77$ & $917 \pm 87$ & Blue & f01-54 & 0 \\
\hline f02-25 & $12: 42: 58.65$ & $+02: 39: 35.2$ & 2.76 & 127.5 & 21.78 & 1.67 & $794 \pm 36$ & $826 \pm 44$ & RedFinal & f01-55 & 0 \\
\hline f02-26 & $12: 42: 58.64$ & $+02: 39: 43.0$ & 2.68 & 125.3 & 21.95 & 1.49 & $730 \pm 29$ & $829 \pm 42$ & BlueFinal & f01-56 & 0 \\
\hline f02-27 & $12: 42: 59.02$ & $+02: 39: 49.6$ & 2.70 & 122.2 & 21.11 & 1.74 & $921 \pm 36$ & $964 \pm 42$ & RedFinal & f01-57 & 0 \\
\hline f02-28 & $12: 42: 56.41$ & $+02: 39: 55.3$ & 2.12 & 129.5 & 22.23 & 1.11 & $1606 \pm 75$ & $1606 \pm 75$ & R2.5 & & 1 \\
\hline f02-29 & $12: 43: 00.54$ & $+02: 40: 02.8$ & 2.93 & 114.6 & 22.38 & 1.41 & $771 \pm 87$ & $771 \pm 87$ & Blue & & 1 \\
\hline f02-30 & $12: 43: 04.37$ & $+02: 40: 08.0$ & 3.79 & 107.4 & 21.03 & 1.59 & $771 \pm 28$ & $771 \pm 28$ & RedFinal & & 1 \\
\hline f02-31 & $12: 42: 58.93$ & $+02: 40: 24.7$ & 2.42 & 110.7 & 21.78 & 1.61 & $772 \pm 39$ & $772 \pm 39$ & R2.5 & & 1 \\
\hline f02-32 & $12: 43: 07.06$ & $+02: 40: 28.5$ & 4.37 & 100.4 & 21.37 & 1.51 & $612 \pm 46$ & $612 \pm 46$ & BlueFinal & & 1 \\
\hline f02-33 & $12: 43: 04.12$ & $+02: 40: 34.1$ & 3.63 & 101.1 & 21.15 & 1.32 & $664 \pm 21$ & $664 \pm 21$ & BlueFinal & & 1 \\
\hline f02-34 & $12: 43: 02.26$ & $+02: 40: 38.1$ & 3.16 & 101.5 & 21.85 & 1.50 & $797 \pm 51$ & $797 \pm 51$ & BlueFinal & & 1 \\
\hline f02-35 & $12: 43: 05.93$ & $+02: 40: 45.5$ & 4.04 & 97.2 & 20.80 & 1.21 & $604 \pm 22$ & $645 \pm 33$ & BlueFinal & f12-33 & 1 \\
\hline f02-37 & $12: 43: 03.83$ & $+02: 40: 56.3$ & 3.50 & 95.4 & 22.28 & 2.01 & $1058 \pm 27$ & $1058 \pm 27$ & RedFinal & & 1 \\
\hline f02-38 & 12:43:01.61 & $+02: 41: 04.1$ & 2.94 & 93.9 & 22.10 & 1.67 & $500 \pm 38$ & $500 \pm 38$ & RedFinal & & 1 \\
\hline f02-39 & $12: 42: 58.36$ & $+02: 41: 09.6$ & 2.12 & 92.9 & 19.14 & 1.72 & $959 \pm 9$ & $959 \pm 9$ & R2.5 & & 1 \\
\hline f02-40 & $12: 43: 06.22$ & $+02: 41: 15.8$ & 4.08 & 90.0 & 22.22 & 1.91 & $867 \pm 27$ & $867 \pm 27$ & RedFinal & & 1 \\
\hline f02-41 & $12: 43: 05.43$ & $+02: 41: 21.0$ & 3.89 & 88.8 & 22.00 & 1.16 & $793 \pm 59$ & $793 \pm 59$ & BlueFinal & & 1 \\
\hline f02-42 & $12: 43: 06.92$ & $+02: 41: 25.6$ & 4.26 & 87.8 & 21.13 & 1.67 & $841 \pm 33$ & $854 \pm 37$ & RedFinal & f12-38 & 1 \\
\hline f02-44 & $12: 43: 00.61$ & $+02: 41: 39.8$ & 2.71 & 81.6 & 22.03 & 1.76 & $805 \pm 26$ & $805 \pm 26$ & RedFinal & & 1 \\
\hline f02-45 & $12: 43: 01.86$ & $+02: 41: 48.0$ & 3.04 & 79.9 & 21.92 & 1.80 & $856 \pm 33$ & $856 \pm 33$ & RedFinal & & 1 \\
\hline f02-47 & $12: 43: 02.36$ & $+02: 41: 59.5$ & 3.20 & 76.9 & 21.96 & 1.61 & $848 \pm 30$ & $848 \pm 30$ & RedFinal & & 1 \\
\hline f02-48 & 12:43:09.04 & $+02: 42: 06.1$ & 4.86 & 80.1 & 20.02 & 1.54 & $697 \pm 14$ & $713 \pm 30$ & BlueFinal & $6.1: 92, \mathrm{f} 12-44$ & 1 \\
\hline f02-50 & 12:43:04.95 & $+02: 42: 16.6$ & 3.90 & 75.0 & 21.64 & 1.72 & $692 \pm 28$ & $692 \pm 28$ & RedFinal & & 1 \\
\hline f02-51 & $12: 42: 55.53$ & $+02: 42: 23.7$ & 1.81 & 51.4 & 22.29 & 1.19 & $1028 \pm 50$ & $1028 \pm 50$ & R2.5 & & 1 \\
\hline f02-52 & $12: 43: 08.27$ & $+02: 42: 26.7$ & 4.74 & 75.6 & 21.84 & 1.14 & $1150 \pm 64$ & $1150 \pm 64$ & BlueFinal & & 1 \\
\hline f02-53 & $12: 42: 59.09$ & $+02: 42: 35.2$ & 2.65 & 60.2 & 21.87 & 1.80 & $1047 \pm 56$ & $1047 \pm 56$ & RedFinal & & 1 \\
\hline f02-55 & $12: 43: 06.79$ & $+02: 42: 43.9$ & 4.47 & 70.9 & 20.13 & 1.15 & $878 \pm 24$ & $881 \pm 35$ & BlueFinal & $6.2: 108$ & 1 \\
\hline f02-56 & $12: 43: 07.57$ & $+02: 42: 48.7$ & 4.68 & 70.7 & 22.26 & 1.40 & $659 \pm 42$ & $659 \pm 42$ & BlueFinal & & 1 \\
\hline f02-57 & 12:43:01.33 & $+02: 42: 52.1$ & 3.28 & 60.8 & 21.88 & 1.37 & $681 \pm 49$ & $681 \pm 49$ & BlueFinal & & 1 \\
\hline f02-58 & $12: 43: 08.47$ & $+02: 42: 57.9$ & 4.95 & 69.9 & 21.16 & 1.15 & $980 \pm 32$ & $1011 \pm 44$ & BlueFinal & f12-51 & 1 \\
\hline f02-59 & 12:43:08.05 & $+02: 43: 06.9$ & 4.90 & 67.8 & 21.29 & 1.71 & $876 \pm 37$ & $876 \pm 37$ & RedFinal & & 1 \\
\hline f02-60 & $12: 43: 10.45$ & $+02: 43: 14.0$ & 5.50 & 69.1 & 22.32 & 1.76 & $679 \pm 50$ & $730 \pm 61$ & RedFinal & f12-53 & 1 \\
\hline f02-61 & $12: 42: 57.87$ & $+02: 43: 19.3$ & 2.87 & 44.2 & 22.45 & 0.73 & $492 \pm 51$ & $492 \pm 51$ & BlueFinal & & 1 \\
\hline f02-62 & $12: 42: 58.27$ & $+02: 43: 28.1$ & 3.04 & 43.6 & 22.42 & 1.16 & $972 \pm 54$ & $972 \pm 54$ & BlueFinal & & 1 \\
\hline f02-63 & $12: 43: 09.76$ & $+02: 43: 31.9$ & 5.46 & 65.5 & 21.63 & 1.27 & $964 \pm 54$ & $964 \pm 54$ & BlueFinal & & 1 \\
\hline f02-64 & $12: 42: 56.27$ & $+02: 43: 39.5$ & 2.88 & 33.8 & 21.83 & 1.76 & $1149 \pm 35$ & $1057 \pm 69$ & RedFinal & f03-01 & 1 \\
\hline f02-65 & $12: 43: 06.36$ & $+02: 43: 45.6$ & 4.81 & 58.8 & 21.70 & 1.77 & $926 \pm 31$ & $952 \pm 67$ & RedFinal & f03-02, f12-58 & 1 \\
\hline f02-66 & $12: 43: 03.48$ & $+02: 43: 53.5$ & 4.29 & 52.3 & 21.63 & 1.22 & $1124 \pm 104$ & $1042 \pm 125$ & Blue & f03-03 & 1 \\
\hline f02-69 & $12: 43: 03.41$ & $+02: 44: 14.5$ & 4.50 & 48.7 & 22.43 & 1.35 & $729 \pm 81$ & $729 \pm 81$ & Blue & & 1 \\
\hline f03-01 & $12: 42: 56.27$ & $+02: 43: 39.5$ & 2.88 & 33.8 & 21.83 & 1.76 & $965 \pm 60$ & $1057 \pm 69$ & RedFinal & f02-64 & 0 \\
\hline f03-02 & 12:43:06.36 & $+02: 43: 45.6$ & 4.81 & 58.8 & 21.70 & 1.77 & $908 \pm 46$ & $952 \pm 67$ & RedFinal & f02-65, f12-58 & 0 \\
\hline f03-03 & $12: 43: 03.48$ & $+02: 43: 53.5$ & 4.29 & 52.3 & 21.63 & 1.22 & $960 \pm 69$ & $1042 \pm 125$ & Blue & f02-66 & 0 \\
\hline f03-06 & 12:43:07.07 & $+02: 44: 05.9$ & 5.14 & 56.6 & 22.44 & 1.14 & $661 \pm 63$ & $661 \pm 63$ & BlueFinal & & 1 \\
\hline f03-08 & $12: 42: 58.04$ & $+02: 44: 18.5$ & 3.66 & 33.9 & 21.32 & 1.17 & $1216 \pm 69$ & $1216 \pm 69$ & Blue & & 1 \\
\hline f03-09 & $12: 43: 00.00$ & $+02: 44: 24.1$ & 4.03 & 38.9 & 22.20 & 1.67 & $350 \pm 69$ & $350 \pm 69$ & Red & & 1 \\
\hline f03-10 & $12: 43: 03.97$ & $+02: 44: 26.9$ & 4.75 & 47.9 & 20.39 & 1.66 & $824 \pm 23$ & $824 \pm 23$ & RedFinal & & 1 \\
\hline f03-12 & $12: 42: 55.55$ & $+02: 44: 39.2$ & 3.67 & 22.7 & 20.45 & 1.26 & $1048 \pm 61$ & $1045 \pm 78$ & Blue & $5.1: 22$ & 1 \\
\hline f03-14 & $12: 43: 01.73$ & $+02: 44: 55.6$ & 4.71 & 39.0 & 21.91 & 1.39 & $688 \pm 89$ & $688 \pm 89$ & Blue & & 1 \\
\hline f03-16 & 12:43:03.94 & $+02: 45: 07.4$ & 5.22 & 42.3 & 22.39 & 1.61 & $1159 \pm 58$ & $1159 \pm 58$ & RedFinal & & 1 \\
\hline f03-17 & $12: 43: 04.28$ & $+02: 45: 17.5$ & 5.40 & 41.8 & 22.19 & 1.21 & $984 \pm 71$ & $984 \pm 71$ & Blue & & 1 \\
\hline f03-20 & 12:43:05.34 & $+02: 45: 31.3$ & 5.75 & 42.2 & 21.86 & 1.79 & $795 \pm 57$ & $825 \pm 64$ & RedFinal & $5.1: 38$ & 1 \\
\hline f03-23 & $12: 42: 56.65$ & $+02: 45: 46.4$ & 4.81 & 20.6 & 21.69 & 1.71 & $647 \pm 46$ & $647 \pm 46$ & RedFinal & & 1 \\
\hline f03-25 & $12: 43: 01.22$ & $+02: 45: 54.9$ & 5.44 & 31.4 & 22.09 & 1.45 & $1153 \pm 100$ & $1153 \pm 100$ & Blue & & 1 \\
\hline f03-26 & $12: 43: 10.38$ & $+02: 46: 03.7$ & 7.02 & 46.9 & 21.14 & 1.62 & $990 \pm 27$ & $990 \pm 27$ & RedFinal & & 1 \\
\hline f03-27 & $12: 43: 07.48$ & $+02: 46: 10.2$ & 6.59 & 41.9 & 20.89 & 1.17 & $898 \pm 43$ & $898 \pm 43$ & BlueFinal & & 1 \\
\hline f03-32 & $12: 43: 08.34$ & $+02: 46: 49.9$ & 7.23 & 39.6 & 19.33 & 1.40 & $746 \pm 42$ & $721 \pm 45$ & BlueFinal & $5.1: 52$ & 1 \\
\hline f03-35 & 12:43:01.93 & $+02: 47: 10.0$ & 6.62 & 27.0 & 22.00 & 1.26 & $420 \pm 99$ & $420 \pm 99$ & Blue & & 1 \\
\hline f03-36 & $12: 43: 02.28$ & $+02: 47: 14.1$ & 6.72 & 27.4 & 20.67 & 1.32 & $845 \pm 35$ & $845 \pm 35$ & BlueFinal & & 1 \\
\hline f03-40 & $12: 43: 11.16$ & $+02: 47: 32.2$ & 8.22 & 40.3 & 20.19 & 1.30 & $1110 \pm 34$ & $1110 \pm 34$ & BlueFinal & & 1 \\
\hline f03-48 & $12: 43: 09.30$ & $+02: 47: 40.9$ & 8.04 & 37.1 & 22.24 & 1.73 & $1186 \pm 68$ & $1186 \pm 68$ & RedFinal & & 1 \\
\hline f03-54 & $12: 43: 01.02$ & $+02: 49: 19.9$ & 8.53 & 19.0 & 21.39 & 1.28 & $1093 \pm 91$ & $1054 \pm 102$ & Blue & $5.1: 91$ & 1 \\
\hline f03-57 & $12: 43: 04.31$ & $+02: 49: 43.0$ & 9.19 & 23.1 & 21.36 & 1.34 & $963 \pm 76$ & $963 \pm 76$ & Blue & & 1 \\
\hline f03-58 & $12: 42: 55.70$ & $+02: 49: 49.7$ & 8.68 & 9.6 & 21.09 & 1.04 & $604 \pm 100$ & $604 \pm 100$ & Blue & & 1 \\
\hline f04-06 & $12: 42: 46.57$ & $+02: 46: 22.0$ & 5.17 & -9.2 & 21.57 & 1.74 & $759 \pm 40$ & $806 \pm 49$ & RedFinal & f05-57 & 1 \\
\hline f04-11 & $12: 42: 42.06$ & $+02: 46: 48.9$ & 5.88 & -19.4 & 20.24 & 1.61 & $942 \pm 20$ & $978 \pm 27$ & RedFinal & f05-61, f13-13 & 1 \\
\hline
\end{tabular}


Table C.1. continued.

\begin{tabular}{|c|c|c|c|c|c|c|c|c|c|c|c|}
\hline ID & $\begin{array}{c}\text { RA } \\
(\mathrm{J} 2000)\end{array}$ & $\begin{array}{c}\text { Dec } \\
(\mathrm{J} 2000)\end{array}$ & $\begin{array}{c}R_{4636} \\
\text { [arcmin] }\end{array}$ & $\begin{array}{c}\text { PA } \\
{[\mathrm{deg}]}\end{array}$ & $\begin{array}{c}m_{R} \\
{[\mathrm{mag}]}\end{array}$ & $\begin{array}{l}C-R \\
{[\mathrm{mag}]}\end{array}$ & $\begin{array}{c}v_{\text {spec }} \\
{\left[\mathrm{km} \mathrm{s}^{-1}\right]}\end{array}$ & $\begin{array}{c}v_{\text {final }} \\
{\left[\mathrm{km} \mathrm{s}^{-1}\right]}\end{array}$ & Sample & Cross-Identification(s) & $1 \mathrm{st}$ \\
\hline f04-12 & $12: 42: 49.93$ & $+02: 46: 54.7$ & 5.65 & 0.2 & 22.02 & 1.74 & $1045 \pm 59$ & $1087 \pm 68$ & RedFinal & f05-62 & 1 \\
\hline f04-17 & $12: 42: 54.65$ & $+02: 47: 27.1$ & 6.30 & 10.9 & 22.08 & 1.60 & $704 \pm 42$ & $704 \pm 42$ & RedFinal & & 1 \\
\hline f04-20 & $12: 42: 38.86$ & $+02: 47: 48.1$ & 7.09 & -22.8 & 22.11 & 1.28 & $625 \pm 82$ & $644 \pm 91$ & Blue & f13-24 & 1 \\
\hline f04-21 & $12: 42: 41.06$ & $+02: 47: 52.2$ & 6.96 & -18.4 & 21.90 & 1.63 & $567 \pm 78$ & $595 \pm 86$ & RedFinal & f13-25 & 1 \\
\hline f04-22 & $12: 42: 49.43$ & $+02: 48: 04.0$ & 6.80 & -0.9 & 21.84 & 1.63 & $854 \pm 59$ & $854 \pm 59$ & RedFinal & & 1 \\
\hline f04-28 & $12: 42: 41.80$ & $+02: 48: 37.5$ & 7.63 & -15.3 & 20.71 & 1.50 & $1051 \pm 24$ & $1051 \pm 24$ & BlueFinal & & 1 \\
\hline f04-31 & $12: 42: 44.13$ & $+02: 49: 06.2$ & 7.97 & -10.4 & 22.00 & 1.17 & $474 \pm 43$ & $474 \pm 43$ & Blue & & 1 \\
\hline f04-34 & $12: 42: 45.08$ & $+02: 49: 29.2$ & 8.31 & -8.3 & 21.94 & 1.70 & $825 \pm 56$ & $825 \pm 56$ & RedFinal & & 1 \\
\hline f04-36 & $12: 42: 51.62$ & $+02: 49: 41.8$ & 8.44 & 3.0 & 21.70 & 1.52 & $872 \pm 45$ & $872 \pm 45$ & BlueFinal & & 1 \\
\hline f04-37 & $12: 42: 39.25$ & $+02: 49: 52.9$ & 9.01 & -17.1 & 22.07 & 1.25 & $817 \pm 48$ & $817 \pm 48$ & BlueFinal & & 1 \\
\hline f04-38 & $12: 42: 42.85$ & $+02: 49: 56.9$ & 8.86 & -11.4 & 21.05 & 1.41 & $942 \pm 45$ & $942 \pm 45$ & BlueFinal & & 1 \\
\hline f04-45 & $12: 42: 54.18$ & $+02: 50: 39.7$ & 9.46 & 6.5 & 20.44 & 1.17 & $1146 \pm 47$ & $1146 \pm 47$ & BlueFinal & & 1 \\
\hline f04-49 & $12: 42: 47.11$ & $+02: 51: 02.8$ & 9.80 & -4.0 & 20.78 & 1.22 & $832 \pm 38$ & $832 \pm 38$ & BlueFinal & & 1 \\
\hline f05-01 & $12: 42: 48.24$ & $+02: 40: 10.2$ & 1.17 & -159.6 & 19.61 & 1.43 & $1048 \pm 18$ & $1048 \pm 18$ & $\mathrm{R} 2.5$ & & 1 \\
\hline f05-02 & $12: 42: 39.68$ & $+02: 40: 15.4$ & 2.74 & -111.6 & 20.53 & 1.49 & $909 \pm 30$ & $906 \pm 35$ & BlueFinal & f07-27 & 1 \\
\hline f05-03 & $12: 42: 44.47$ & $+02: 40: 20.4$ & 1.64 & -124.5 & 22.40 & 1.19 & $1193 \pm 54$ & $1193 \pm 54$ & R2.5 & & 1 \\
\hline f05-04 & $12: 42: 47.80$ & $+02: 40: 25.5$ & 0.99 & -148.4 & 22.34 & 1.23 & $542 \pm 68$ & $542 \pm 68$ & R2.5 & & 1 \\
\hline f05-05 & $12: 42: 45.34$ & $+02: 40: 33.9$ & 1.33 & -121.8 & 21.68 & 1.27 & $815 \pm 63$ & $815 \pm 63$ & R2.5 & & 1 \\
\hline f05-06 & $12: 42: 47.24$ & $+02: 40: 40.6$ & 0.88 & -131.9 & 21.71 & 1.53 & $827 \pm 32$ & $827 \pm 32$ & $\mathrm{R} 2.5$ & & 1 \\
\hline f05-07 & $12: 42: 49.17$ & $+02: 40: 47.3$ & 0.51 & -159.9 & 20.65 & 0.94 & $826 \pm 48$ & $826 \pm 48$ & $\mathrm{R} 2.5$ & & 1 \\
\hline f05-08 & $12: 42: 45.98$ & $+02: 40: 54.7$ & 1.03 & -110.1 & 20.32 & 1.51 & $1115 \pm 17$ & $1115 \pm 17$ & $\mathrm{R} 2.5$ & & 1 \\
\hline f05-09 & $12: 42: 41.42$ & $+02: 41: 02.0$ & 2.12 & -96.3 & 22.33 & 1.36 & $528 \pm 41$ & $502 \pm 66$ & R2.5 & f07-33 & 1 \\
\hline f05-10 & $12: 42: 47.08$ & $+02: 41: 07.0$ & 0.71 & -102.2 & 20.58 & 1.16 & $859 \pm 40$ & $859 \pm 40$ & R2.5 & & 1 \\
\hline f05-11 & $12: 42: 45.14$ & $+02: 41: 14.5$ & 1.18 & -91.2 & 21.05 & 1.34 & $810 \pm 23$ & $810 \pm 23$ & R2.5 & & 1 \\
\hline f05-12 & $12: 42: 47.50$ & $+02: 41: 20.2$ & 0.60 & -83.3 & 21.25 & 1.12 & $640 \pm 38$ & $640 \pm 38$ & R2.5 & & 1 \\
\hline f05-13 & $12: 42: 43.58$ & $+02: 41: 24.3$ & 1.58 & -85.0 & 21.24 & 1.62 & $1090 \pm 19$ & $1134 \pm 28$ & R2.5 & f07-37 & 1 \\
\hline f05-14 & $12: 42: 40.84$ & $+02: 41: 30.1$ & 2.27 & -84.1 & 22.12 & 1.48 & $1006 \pm 22$ & $1006 \pm 22$ & $\mathrm{R} 2.5$ & & 1 \\
\hline f05-16 & $12: 42: 48.12$ & $+02: 41: 43.5$ & 0.63 & -43.6 & 19.78 & 1.31 & $728 \pm 12$ & $728 \pm 12$ & $\mathrm{R} 2.5$ & & 1 \\
\hline f05-17 & $12: 42: 43.79$ & $+02: 41: 50.1$ & 1.62 & -69.5 & 21.13 & 1.61 & $452 \pm 20$ & $456 \pm 36$ & $\mathrm{R} 2.5$ & $1.2: 83$ & 1 \\
\hline f05-18 & $12: 42: 42.92$ & $+02: 41: 54.9$ & 1.85 & -69.5 & 21.36 & 1.25 & $1244 \pm 19$ & $1244 \pm 19$ & $\mathrm{R} 2.5$ & & 1 \\
\hline f05-19 & $12: 42: 48.60$ & $+02: 42: 04.8$ & 0.87 & -21.3 & 21.38 & 1.33 & $1269 \pm 18$ & $1269 \pm 18$ & R2.5 & & 1 \\
\hline f05-20 & $12: 42: 45.45$ & $+02: 42: 09.1$ & 1.41 & -51.3 & 21.16 & 1.54 & $427 \pm 93$ & $509 \pm 102$ & R2.5 & f07-45 & 1 \\
\hline f05-21 & $12: 42: 46.28$ & $+02: 42: 15.6$ & 1.34 & -42.1 & 21.38 & 1.87 & $1055 \pm 43$ & $1055 \pm 43$ & $\mathrm{R} 2.5$ & & 1 \\
\hline f05-22 & $12: 42: 44.40$ & $+02: 42: 20.3$ & 1.74 & -51.9 & 21.32 & 1.69 & $803 \pm 26$ & $823 \pm 33$ & $\mathrm{R} 2.5$ & f07-47 & 1 \\
\hline f05-23 & $12: 42: 48.53$ & $+02: 42: 27.3$ & 1.23 & -15.7 & 20.32 & 1.01 & $787 \pm 37$ & $787 \pm 37$ & $\mathrm{R} 2.5$ & & 1 \\
\hline f05-25 & $12: 42: 45.98$ & $+02: 42: 41.4$ & 1.72 & -34.3 & 21.07 & 1.33 & $713 \pm 32$ & $715 \pm 45$ & $\mathrm{R} 2.5$ & f07-51 & 1 \\
\hline f05-26 & $12: 42: 42.40$ & $+02: 42: 46.7$ & 2.40 & -51.0 & 21.41 & 1.54 & $561 \pm 29$ & $600 \pm 38$ & R2.5 & f07-52 & 1 \\
\hline f05-27 & $12: 42: 43.11$ & $+02: 42: 49.9$ & 2.30 & -47.2 & 20.64 & 1.46 & $1013 \pm 20$ & $1019 \pm 26$ & R2.5 & f07-53 & 1 \\
\hline f05-28 & $12: 42: 39.99$ & $+02: 42: 56.9$ & 2.99 & -55.7 & 20.82 & 1.13 & $804 \pm 21$ & $788 \pm 30$ & BlueFinal & f07-54 & 1 \\
\hline f05-29 & $12: 42: 48.14$ & $+02: 43: 17.5$ & 2.07 & -12.0 & 21.79 & 1.19 & $1322 \pm 27$ & $1322 \pm 27$ & $\mathrm{R} 2.5$ & & 1 \\
\hline f05-30 & $12: 42: 50.12$ & $+02: 43: 23.5$ & 2.13 & 1.7 & 21.04 & 1.33 & $753 \pm 28$ & $753 \pm 28$ & $\mathrm{R} 2.5$ & & 1 \\
\hline f05-31 & $12: 42: 45.36$ & $+02: 43: 29.7$ & 2.50 & -26.8 & 22.45 & 1.65 & $986 \pm 56$ & $986 \pm 56$ & $\mathrm{R} 2.5$ & & 1 \\
\hline f05-32 & $12: 42: 50.21$ & $+02: 43: 38.9$ & 2.38 & 2.0 & 21.82 & 1.65 & $740 \pm 30$ & $740 \pm 30$ & $\mathrm{R} 2.5$ & & 1 \\
\hline f05-33 & $12: 42: 49.62$ & $+02: 43: 43.6$ & 2.46 & -1.5 & 20.79 & 2.02 & $638 \pm 17$ & $638 \pm 17$ & $\mathrm{R} 2.5$ & & 1 \\
\hline f05-35 & $12: 42: 47.12$ & $+02: 43: 56.0$ & 2.75 & -14.4 & 19.69 & 1.66 & $1061 \pm 19$ & $1071 \pm 24$ & RedFinal & f07-66 & 1 \\
\hline f05-37 & $12: 42: 51.84$ & $+02: 44: 06.1$ & 2.88 & 9.8 & 21.48 & 1.76 & $932 \pm 24$ & $932 \pm 24$ & RedFinal & & 1 \\
\hline f05-39 & $12: 42: 38.81$ & $+02: 44: 19.7$ & 4.12 & -42.1 & 21.36 & 1.41 & $1172 \pm 36$ & $1192 \pm 45$ & BlueFinal & f07-70 & 1 \\
\hline f05-41 & $12: 42: 42.08$ & $+02: 44: 35.1$ & 3.85 & -30.4 & 21.92 & 1.01 & $991 \pm 89$ & $1038 \pm 102$ & Blue & f07-73 & 1 \\
\hline f05-42 & $12: 42: 51.29$ & $+02: 44: 43.5$ & 3.48 & 5.9 & 21.31 & 1.35 & $822 \pm 24$ & $822 \pm 24$ & BlueFinal & & 1 \\
\hline f05-46 & $12: 42: 43.03$ & $+02: 45: 07.9$ & 4.23 & -23.8 & 22.03 & 1.67 & $937 \pm 36$ & $937 \pm 36$ & RedFinal & & 1 \\
\hline f05-49 & $12: 42: 48.04$ & $+02: 45: 28.5$ & 4.23 & -6.2 & 21.74 & 1.83 & $725 \pm 23$ & $725 \pm 23$ & RedFinal & & 1 \\
\hline f05-52 & $12: 42: 44.04$ & $+02: 45: 47.1$ & 4.75 & -17.9 & 21.79 & 1.19 & $1566 \pm 91$ & $1566 \pm 91$ & Blue & & 1 \\
\hline f05-53 & $12: 42: 45.82$ & $+02: 45: 52.8$ & 4.72 & -12.4 & 21.25 & 1.88 & $727 \pm 22$ & $727 \pm 22$ & RedFinal & & 1 \\
\hline f05-54 & $12: 42: 44.68$ & $+02: 46: 01.6$ & 4.93 & -15.2 & 20.94 & 1.45 & $865 \pm 33$ & $865 \pm 33$ & BlueFinal & & 1 \\
\hline f05-55 & $12: 42: 45.08$ & $+02: 46: 06.7$ & 4.99 & -13.9 & 21.78 & 1.75 & $723 \pm 28$ & $723 \pm 28$ & RedFinal & & 1 \\
\hline f05-56 & $12: 42: 49.20$ & $+02: 46: 17.9$ & 5.03 & -1.9 & 22.49 & 1.49 & $642 \pm 60$ & $642 \pm 60$ & BlueFinal & & 1 \\
\hline f05-57 & $12: 42: 46.57$ & $+02: 46: 22.0$ & 5.17 & -9.2 & 21.57 & 1.74 & $853 \pm 29$ & $806 \pm 49$ & RedFinal & f04-06 & 0 \\
\hline f05-58 & $12: 42: 47.77$ & $+02: 46: 27.3$ & 5.21 & -5.8 & 22.22 & 1.45 & $688 \pm 43$ & $688 \pm 43$ & BlueFinal & & 1 \\
\hline f05-61 & $12: 42: 42.06$ & $+02: 46: 48.9$ & 5.88 & -19.4 & 20.24 & 1.61 & $1015 \pm 14$ & $978 \pm 27$ & RedFinal & f13-13, f04-11 & 0 \\
\hline f05-62 & $12: 42: 49.93$ & $+02: 46: 54.7$ & 5.65 & 0.2 & 22.02 & 1.74 & $1130 \pm 35$ & $1087 \pm 68$ & RedFinal & f04-12 & 0 \\
\hline f06-13 & $12: 42: 49.70$ & $+02: 33: 16.8$ & 7.99 & -179.7 & 22.44 & 1.48 & $874 \pm 72$ & $874 \pm 72$ & Blue & & 1 \\
\hline f06-15 & $12: 42: 58.79$ & $+02: 33: 30.4$ & 8.07 & 164.0 & 22.20 & 1.77 & $927 \pm 40$ & $899 \pm 53$ & RedFinal & f01-07 & 0 \\
\hline f06-16 & $12: 43: 01.37$ & $+02: 33: 35.0$ & 8.20 & 159.5 & 19.14 & 1.66 & $1110 \pm 13$ & $1110 \pm 13$ & RedFinal & & 1 \\
\hline f06-19 & $12: 42: 49.13$ & $+02: 33: 49.7$ & 7.44 & -178.6 & 21.55 & 1.87 & $963 \pm 38$ & $963 \pm 38$ & RedFinal & & 1 \\
\hline f06-22 & $12: 42: 46.93$ & $+02: 34: 11.1$ & 7.12 & -174.1 & 22.11 & 1.69 & $951 \pm 67$ & $951 \pm 67$ & RedFinal & & 1 \\
\hline f06-24 & $12: 42: 54.40$ & $+02: 34: 25.3$ & 6.94 & 170.6 & 21.07 & 1.49 & $1082 \pm 31$ & $1082 \pm 31$ & BlueFinal & & 1 \\
\hline
\end{tabular}


Y. Schuberth et al.: Dynamics of the NGC 4636 globular cluster system. II.

Table C.1. continued.

\begin{tabular}{|c|c|c|c|c|c|c|c|c|c|c|c|}
\hline ID & $\begin{array}{c}\mathrm{RA} \\
(\mathrm{J} 2000)\end{array}$ & $\begin{array}{c}\text { Dec } \\
(\mathrm{J} 2000)\end{array}$ & $\begin{array}{c}R_{4636} \\
\text { [arcmin] }\end{array}$ & $\begin{array}{c}\text { PA } \\
{[\mathrm{deg}]}\end{array}$ & $\begin{array}{c}m_{R} \\
{[\mathrm{mag}]}\end{array}$ & $\begin{array}{l}C-R \\
{[\mathrm{mag}]}\end{array}$ & $\begin{array}{c}v_{\mathrm{spec}} \\
{\left[\mathrm{km} \mathrm{s}^{-1}\right]}\end{array}$ & $\begin{array}{c}v_{\text {final }} \\
{\left[\mathrm{km} \mathrm{s}^{-1}\right]}\end{array}$ & Sample & Cross-Identification(s) & $1 \mathrm{st}$ \\
\hline f06-25 & $12: 43: 02.07$ & $+02: 34: 33.2$ & 7.37 & 155.6 & 21.88 & 1.17 & $871 \pm 62$ & $859 \pm 74$ & Blue & f01-14 & 0 \\
\hline f06-28 & $12: 42: 53.37$ & $+02: 35: 04.8$ & 6.25 & 172.0 & 20.32 & 1.31 & $988 \pm 22$ & $988 \pm 22$ & BlueFinal & & 1 \\
\hline f06-29 & $12: 42: 53.97$ & $+02: 35: 10.8$ & 6.17 & 170.5 & 21.12 & 1.39 & $770 \pm 32$ & $770 \pm 32$ & BlueFinal & & 1 \\
\hline f06-30 & $12: 42: 55.56$ & $+02: 35: 17.3$ & 6.14 & 166.6 & 20.06 & 1.62 & $879 \pm 12$ & $879 \pm 12$ & RedFinal & & 1 \\
\hline f06-33 & $12: 43: 00.16$ & $+02: 35: 32.3$ & 6.28 & 155.8 & 20.10 & 1.70 & $830 \pm 17$ & $830 \pm 17$ & RedFinal & & 1 \\
\hline f06-36 & $12: 42: 55.76$ & $+02: 35: 49.6$ & 5.64 & 164.9 & 22.13 & 1.47 & $1159 \pm 47$ & $1159 \pm 47$ & BlueFinal & & 1 \\
\hline f06-37 & $12: 42: 54.61$ & $+02: 35: 56.5$ & 5.45 & 167.5 & 20.40 & 1.22 & $1138 \pm 36$ & $1138 \pm 36$ & BlueFinal & & 1 \\
\hline f06-38 & $12: 42: 49.71$ & $+02: 36: 02.9$ & 5.22 & -179.6 & 20.70 & 1.84 & $733 \pm 23$ & $693 \pm 37$ & RedFinal & $1.3: 3$ & 1 \\
\hline f06-41 & $12: 42: 55.69$ & $+02: 36: 22.4$ & 5.10 & 163.5 & 21.86 & 1.71 & $1135 \pm 33$ & $1135 \pm 33$ & RedFinal & & 1 \\
\hline f06-43 & $12: 42: 52.63$ & $+02: 36: 31.5$ & 4.79 & 171.7 & 21.44 & 1.35 & $1125 \pm 41$ & $1125 \pm 41$ & BlueFinal & & 1 \\
\hline f06-45 & $12: 42: 55.75$ & $+02: 36: 45.2$ & 4.75 & 162.0 & 22.48 & 1.40 & $750 \pm 67$ & $750 \pm 67$ & Blue & & 1 \\
\hline f06-46 & $12: 42: 54.93$ & $+02: 36: 52.8$ & 4.57 & 163.9 & 21.85 & 1.94 & $1163 \pm 38$ & $1163 \pm 38$ & RedFinal & & 1 \\
\hline f06-47 & $12: 42: 54.90$ & $+02: 36: 57.7$ & 4.48 & 163.7 & 21.82 & 1.31 & $973 \pm 58$ & $973 \pm 58$ & BlueFinal & & 1 \\
\hline f06-48 & $12: 42: 53.42$ & $+02: 37: 01.2$ & 4.34 & 168.2 & 20.02 & 1.62 & $1149 \pm 17$ & $1124 \pm 23$ & RedFinal & $1.3: 19$ & 1 \\
\hline f06-50 & $12: 42: 46.89$ & $+02: 37: 15.2$ & 4.08 & -169.5 & 20.50 & 1.73 & $988 \pm 17$ & $944 \pm 30$ & RedFinal & $1.3: 23$ & 1 \\
\hline f06-54 & $12: 42: 54.82$ & $+02: 37: 32.1$ & 3.93 & 161.7 & 22.02 & 1.48 & $1419 \pm 50$ & $1419 \pm 50$ & Blue & & 1 \\
\hline f06-55 & $12: 43: 03.71$ & $+02: 37: 37.2$ & 5.02 & 136.5 & 20.18 & 1.54 & $887 \pm 17$ & $840 \pm 35$ & BlueFinal & f01-38, f02-04 & 0 \\
\hline f06-56 & $12: 42: 55.12$ & $+02: 37: 43.8$ & 3.77 & 159.7 & 20.61 & 1.65 & $943 \pm 22$ & $909 \pm 37$ & RedFinal & $1.3: 31$ & 1 \\
\hline f06-61 & $12: 42: 56.18$ & $+02: 38: 11.2$ & 3.46 & 152.9 & 21.01 & 1.22 & $1020 \pm 39$ & $932 \pm 47$ & BlueFinal & f02-10 & 0 \\
\hline f06-62 & $12: 42: 50.94$ & $+02: 38: 14.6$ & 3.04 & 174.9 & 21.71 & 1.78 & $976 \pm 37$ & $976 \pm 37$ & RedFinal & & 1 \\
\hline f06-63 & $12: 42: 52.53$ & $+02: 38: 18.9$ & 3.03 & 167.3 & 21.68 & 1.70 & $1010 \pm 54$ & $1010 \pm 54$ & RedFinal & & 1 \\
\hline f06-64 & $12: 42: 53.47$ & $+02: 38: 24.3$ & 3.00 & 162.6 & 21.19 & 1.31 & $1297 \pm 41$ & $1297 \pm 41$ & BlueFinal & & 1 \\
\hline f06-65 & $12: 42: 57.50$ & $+02: 38: 30.2$ & 3.36 & 145.4 & 19.83 & 1.27 & $1336 \pm 22$ & $1280 \pm 30$ & BlueFinal & f02-14 & 0 \\
\hline f06-68 & $12: 42: 53.28$ & $+02: 38: 44.0$ & 2.67 & 161.4 & 19.64 & 1.18 & $1311 \pm 25$ & $1260 \pm 35$ & BlueFinal & $1.1: 44$ & 1 \\
\hline f06-70 & $12: 42: 55.66$ & $+02: 38: 51.8$ & 2.80 & 149.0 & 20.76 & 1.18 & $837 \pm 29$ & $837 \pm 29$ & BlueFinal & & 1 \\
\hline f06-71 & $12: 42: 50.37$ & $+02: 38: 56.6$ & 2.33 & 176.9 & 21.70 & 1.71 & $1320 \pm 49$ & $1320 \pm 49$ & $\mathrm{R} 2.5$ & & 1 \\
\hline f07-02 & $12: 42: 44.81$ & $+02: 37: 58.1$ & 3.53 & -159.0 & 19.93 & 1.56 & $885 \pm 15$ & $868 \pm 27$ & RedFinal & $1.1: 32$ & 1 \\
\hline f07-05 & $12: 42: 44.36$ & $+02: 38: 13.0$ & 3.35 & -155.7 & 20.16 & 1.45 & $685 \pm 12$ & $676 \pm 27$ & BlueFinal & $1.1: 36$ & 1 \\
\hline f07-06 & $12: 42: 44.23$ & $+02: 38: 20.9$ & 3.24 & -154.2 & 22.15 & 2.10 & $854 \pm 46$ & $854 \pm 46$ & RedFinal & & 1 \\
\hline f07-08 & $12: 42: 43.41$ & $+02: 38: 29.7$ & 3.21 & -149.8 & 21.06 & 1.38 & $688 \pm 21$ & $637 \pm 34$ & BlueFinal & $1.1: 40$ & 1 \\
\hline f07-09 & $12: 42: 44.34$ & $+02: 38: 34.3$ & 3.03 & -152.9 & 22.41 & 1.20 & $825 \pm 90$ & $825 \pm 90$ & Blue & & 1 \\
\hline f07-10 & $12: 42: 46.10$ & $+02: 38: 37.3$ & 2.81 & -160.4 & 22.31 & 1.82 & $828 \pm 109$ & $828 \pm 109$ & RedFinal & & 1 \\
\hline f07-12 & $12: 42: 40.78$ & $+02: 38: 48.9$ & 3.34 & -137.2 & 21.70 & 1.27 & $841 \pm 42$ & $841 \pm 42$ & BlueFinal & & 1 \\
\hline f07-13 & $12: 42: 40.52$ & $+02: 38: 55.0$ & 3.31 & -135.2 & 20.28 & 1.35 & $1219 \pm 14$ & $1219 \pm 14$ & BlueFinal & & 1 \\
\hline f07-15 & $12: 42: 44.72$ & $+02: 39: 05.1$ & 2.53 & -149.5 & 20.30 & 1.85 & $1138 \pm 14$ & $1115 \pm 25$ & RedFinal & $1.1: 47$ & 1 \\
\hline f07-17 & $12: 42: 46.04$ & $+02: 39: 15.9$ & 2.22 & -154.5 & 21.32 & 1.84 & $744 \pm 19$ & $747 \pm 29$ & R2.5 & $1.3: 52$ & 1 \\
\hline f07-18 & $12: 42: 36.69$ & $+02: 39: 21.7$ & 3.80 & -120.1 & 20.69 & 1.67 & $1114 \pm 34$ & $1098 \pm 36$ & RedFinal & f08-37 & 1 \\
\hline f07-19 & $12: 42: 44.17$ & $+02: 39: 27.1$ & 2.31 & -141.9 & 19.80 & 1.33 & $1228 \pm 14$ & $1219 \pm 23$ & R2.5 & $1.1: 53$ & 1 \\
\hline f07-20 & $12: 42: 40.52$ & $+02: 39: 31.2$ & 2.92 & -126.8 & 21.11 & 1.15 & $969 \pm 34$ & $969 \pm 34$ & BlueFinal & & 1 \\
\hline f07-21 & $12: 42: 34.93$ & $+02: 39: 39.5$ & 4.06 & -113.3 & 22.01 & 1.20 & $1090 \pm 40$ & $1084 \pm 69$ & Blue & f08-40 & 1 \\
\hline f07-23 & $12: 42: 38.45$ & $+02: 39: 51.5$ & 3.18 & -116.3 & 21.93 & 1.75 & $920 \pm 54$ & $865 \pm 70$ & RedFinal & f08-42 & 1 \\
\hline f07-25 & $12: 42: 40.01$ & $+02: 39: 59.9$ & 2.77 & -117.3 & 21.91 & 1.35 & $1042 \pm 49$ & $1042 \pm 49$ & BlueFinal & & 1 \\
\hline f07-26 & $12: 42: 47.25$ & $+02: 40: 10.0$ & 1.28 & -149.3 & 20.51 & 1.08 & $1348 \pm 24$ & $1348 \pm 24$ & R2.5 & & 1 \\
\hline f07-27 & $12: 42: 39.68$ & $+02: 40: 15.4$ & 2.74 & -111.6 & 20.53 & 1.49 & $902 \pm 19$ & $906 \pm 35$ & BlueFinal & f05-02 & 0 \\
\hline f07-28 & $12: 42: 36.86$ & $+02: 40: 18.6$ & 3.39 & -106.4 & 21.92 & 1.77 & $867 \pm 37$ & $866 \pm 49$ & RedFinal & f08-47 & 1 \\
\hline f07-29 & $12: 42: 39.30$ & $+02: 40: 25.5$ & 2.77 & -107.7 & 21.78 & 1.04 & $664 \pm 80$ & $679 \pm 95$ & Blue & f08-48 & 1 \\
\hline f07-30 & $12: 42: 42.58$ & $+02: 40: 32.0$ & 1.96 & -111.9 & 20.06 & 1.55 & $933 \pm 15$ & $918 \pm 21$ & R2.5 & $1.1: 71$ & 1 \\
\hline f07-31 & $12: 42: 30.93$ & $+02: 40: 38.6$ & 4.77 & -97.5 & 21.81 & 1.81 & $1009 \pm 24$ & $978 \pm 44$ & RedFinal & f10-16 & 1 \\
\hline f07-32 & $12: 42: 44.55$ & $+02: 40: 54.8$ & 1.37 & -104.9 & 21.03 & 1.25 & $1222 \pm 24$ & $1222 \pm 24$ & R2.5 & & 1 \\
\hline f07-33 & $12: 42: 41.42$ & $+02: 41: 02.0$ & 2.12 & -96.3 & 22.33 & 1.36 & $477 \pm 52$ & $502 \pm 66$ & R2.5 & f05-09 & 0 \\
\hline f07-34 & $12: 42: 36.07$ & $+02: 41: 07.1$ & 3.45 & -92.5 & 22.32 & 1.27 & $976 \pm 111$ & $954 \pm 139$ & Blue & f08-56 & 1 \\
\hline f07-35 & $12: 42: 42.35$ & $+02: 41: 15.1$ & 1.88 & -90.5 & 19.22 & 1.63 & $1091 \pm 24$ & $1059 \pm 29$ & $\mathrm{R} 2.5$ & $1.2: 75$ & 1 \\
\hline f07-36 & $12: 42: 43.71$ & $+02: 41: 19.0$ & 1.54 & -88.1 & 20.80 & 0.98 & $1177 \pm 32$ & $1182 \pm 48$ & $\mathrm{R} 2.5$ & $1.2: 76$ & 1 \\
\hline f07-37 & $12: 42: 43.58$ & $+02: 41: 24.3$ & 1.58 & -85.0 & 21.24 & 1.62 & $1177 \pm 20$ & $1134 \pm 28$ & R2.5 & f05-13 & 0 \\
\hline f07-38 & $12: 42: 38.16$ & $+02: 41: 30.5$ & 2.93 & -85.3 & 21.62 & 1.14 & $889 \pm 37$ & $847 \pm 55$ & BlueFinal & f08-61 & 1 \\
\hline f07-39 & $12: 42: 37.24$ & $+02: 41: 36.8$ & 3.17 & -83.7 & 22.07 & 1.70 & $815 \pm 43$ & $812 \pm 55$ & RedFinal & f08-62 & 1 \\
\hline f07-40 & $12: 42: 32.85$ & $+02: 41: 42.3$ & 4.27 & -84.1 & 21.73 & 1.17 & $1110 \pm 51$ & $1110 \pm 51$ & BlueFinal & & 1 \\
\hline f07-42 & $12: 42: 40.67$ & $+02: 41: 52.1$ & 2.37 & -75.3 & 20.32 & 1.28 & $1254 \pm 26$ & $1254 \pm 26$ & R2.5 & & 1 \\
\hline f07-43 & $12: 42: 33.87$ & $+02: 41: 57.7$ & 4.06 & -80.1 & 21.76 & 1.17 & $791 \pm 45$ & $770 \pm 111$ & Blue & f08-66 & 1 \\
\hline f07-44 & $12: 42: 37.37$ & $+02: 42: 03.3$ & 3.22 & -75.8 & 22.24 & 1.79 & $789 \pm 34$ & $789 \pm 34$ & RedFinal & & 1 \\
\hline f07-45 & $12: 42: 45.45$ & $+02: 42: 09.1$ & 1.41 & -51.3 & 21.16 & 1.54 & $591 \pm 43$ & $509 \pm 102$ & $\mathrm{R} 2.5$ & f05-20 & 0 \\
\hline f07-46 & $12: 42: 40.47$ & $+02: 42: 14.1$ & 2.54 & -67.6 & 22.09 & 0.99 & $776 \pm 40$ & $776 \pm 40$ & BlueFinal & & 1 \\
\hline f07-47 & $12: 42: 44.40$ & $+02: 42: 20.3$ & 1.74 & -51.9 & 21.32 & 1.69 & $842 \pm 22$ & $823 \pm 33$ & R2.5 & f05-22 & 0 \\
\hline f07-49 & $12: 42: 36.70$ & $+02: 42: 30.3$ & 3.51 & -69.4 & 22.32 & 1.72 & $807 \pm 36$ & $807 \pm 36$ & RedFinal & & 1 \\
\hline f07-50 & $12: 42: 33.20$ & $+02: 42: 33.0$ & 4.36 & -72.9 & 21.72 & 1.74 & $720 \pm 19$ & $720 \pm 19$ & RedFinal & & 1 \\
\hline f07-51 & $12: 42: 45.98$ & $+02: 42: 41.4$ & 1.72 & -34.3 & 21.07 & 1.33 & $717 \pm 31$ & $715 \pm 45$ & $\mathrm{R} 2.5$ & f05-25 & 0 \\
\hline
\end{tabular}


Table C.1. continued.

\begin{tabular}{|c|c|c|c|c|c|c|c|c|c|c|c|}
\hline ID & $\begin{array}{c}\text { RA } \\
(\mathrm{J} 2000)\end{array}$ & $\begin{array}{c}\text { Dec } \\
(\mathrm{J} 2000)\end{array}$ & $\begin{array}{c}R_{4636} \\
\text { [arcmin] }\end{array}$ & $\begin{array}{c}\text { PA } \\
{[\mathrm{deg}]}\end{array}$ & $\begin{array}{c}m_{R} \\
{[\mathrm{mag}]}\end{array}$ & $\begin{array}{l}C-R \\
{[\mathrm{mag}]}\end{array}$ & $\begin{array}{c}v_{\mathrm{spec}} \\
{\left[\mathrm{km} \mathrm{s}^{-1}\right]}\end{array}$ & $\begin{array}{c}v_{\text {final }} \\
{\left[\mathrm{km} \mathrm{s}^{-1}\right]}\end{array}$ & Sample & Cross-Identification(s) & $1 \mathrm{st}$ \\
\hline f07-52 & $12: 42: 42.40$ & $+02: 42: 46.7$ & 2.40 & -51.0 & 21.41 & 1.54 & $639 \pm 24$ & $600 \pm 38$ & $\mathrm{R} 2.5$ & f05-26 & 0 \\
\hline f07-53 & $12: 42: 43.11$ & $+02: 42: 49.9$ & 2.30 & -47.2 & 20.64 & 1.46 & $1026 \pm 17$ & $1019 \pm 26$ & R2.5 & f05-27 & 0 \\
\hline f07-54 & $12: 42: 39.99$ & $+02: 42: 56.9$ & 2.99 & -55.7 & 20.82 & 1.13 & $773 \pm 22$ & $788 \pm 30$ & BlueFinal & f05-28 & 0 \\
\hline f07-55 & $12: 42: 41.73$ & $+02: 43: 01.2$ & 2.68 & -49.2 & 21.33 & 1.07 & $767 \pm 37$ & $767 \pm 37$ & BlueFinal & & 1 \\
\hline f07-57 & $12: 42: 45.71$ & $+02: 43: 13.5$ & 2.22 & -27.9 & 21.07 & 1.88 & $1243 \pm 15$ & $1243 \pm 15$ & $\mathrm{R} 2.5$ & & 1 \\
\hline f07-58 & $12: 42: 39.12$ & $+02: 43: 16.8$ & 3.36 & -53.1 & 22.36 & 1.89 & $1009 \pm 34$ & $1009 \pm 34$ & RedFinal & & 1 \\
\hline f07-60 & $12: 42: 43.63$ & $+02: 43: 24.5$ & 2.65 & -36.0 & 21.40 & 1.42 & $697 \pm 15$ & $697 \pm 15$ & BlueFinal & & 1 \\
\hline f07-61 & $12: 42: 43.90$ & $+02: 43: 28.1$ & 2.66 & -34.1 & 21.65 & 1.38 & $996 \pm 43$ & $996 \pm 43$ & BlueFinal & & 1 \\
\hline f07-62 & $12: 42: 45.09$ & $+02: 43: 35.5$ & 2.61 & -27.2 & 19.97 & 1.32 & $1111 \pm 14$ & $1111 \pm 14$ & BlueFinal & & 1 \\
\hline f07-63 & $12: 42: 30.46$ & $+02: 43: 40.4$ & 5.41 & -63.6 & 21.84 & 1.07 & $1145 \pm 57$ & $1165 \pm 79$ & Blue & f10-44 & 1 \\
\hline f07-64 & $12: 42: 37.97$ & $+02: 43: 47.9$ & 3.90 & -49.6 & 22.12 & 1.68 & $1003 \pm 64$ & $1003 \pm 64$ & RedFinal & & 1 \\
\hline f07-65 & $12: 42: 34.72$ & $+02: 43: 50.8$ & 4.58 & -55.7 & 21.20 & 1.40 & $797 \pm 19$ & $797 \pm 19$ & BlueFinal & & 1 \\
\hline f07-66 & $12: 42: 47.12$ & $+02: 43: 56.0$ & 2.75 & -14.4 & 19.69 & 1.66 & $1081 \pm 15$ & $1071 \pm 24$ & RedFinal & f05-35 & 0 \\
\hline f07-68 & $12: 42: 41.81$ & $+02: 44: 07.3$ & 3.49 & -35.2 & 21.49 & 1.54 & $1091 \pm 22$ & $1091 \pm 22$ & BlueFinal & & 1 \\
\hline f07-69 & $12: 42: 33.04$ & $+02: 44: 15.9$ & 5.16 & -54.5 & 21.95 & 1.89 & $916 \pm 27$ & $916 \pm 27$ & RedFinal & & 1 \\
\hline f07-70 & $12: 42: 38.81$ & $+02: 44: 19.7$ & 4.12 & -42.1 & 21.36 & 1.41 & $1211 \pm 28$ & $1192 \pm 45$ & BlueFinal & f05-39 & 0 \\
\hline f07-72 & $12: 42: 35.06$ & $+02: 44: 31.4$ & 4.93 & -48.6 & 21.83 & 1.72 & $1196 \pm 31$ & $1144 \pm 53$ & RedFinal & $3.2: 31$ & 1 \\
\hline f07-73 & $12: 42: 42.08$ & $+02: 44: 35.1$ & 3.85 & -30.4 & 21.92 & 1.01 & $1084 \pm 49$ & $1038 \pm 102$ & Blue & f05-41 & 0 \\
\hline f07-75 & $12: 42: 43.79$ & $+02: 44: 47.0$ & 3.83 & -23.4 & 22.17 & 1.23 & $1098 \pm 57$ & $1098 \pm 57$ & BlueFinal & & 1 \\
\hline f08-02 & $12: 42: 31.72$ & $+02: 35: 11.6$ & 7.58 & -143.3 & 21.56 & 1.30 & $830 \pm 33$ & $830 \pm 33$ & BlueFinal & & 1 \\
\hline f08-07 & $12: 42: 38.67$ & $+02: 35: 41.2$ & 6.24 & -153.4 & 21.48 & 1.59 & $1001 \pm 43$ & $1001 \pm 43$ & RedFinal & & 1 \\
\hline f08-14 & $12: 42: 27.50$ & $+02: 36: 28.8$ & 7.36 & -130.6 & 22.11 & 1.52 & $1006 \pm 40$ & $1006 \pm 40$ & BlueFinal & & 1 \\
\hline f08-18 & $12: 42: 30.80$ & $+02: 36: 54.2$ & 6.46 & -132.5 & 21.33 & 1.62 & $900 \pm 24$ & $900 \pm 24$ & RedFinal & & 1 \\
\hline f08-24 & $12: 42: 37.91$ & $+02: 37: 37.0$ & 4.72 & -140.7 & 22.72 & 1.62 & $1183 \pm 98$ & $1183 \pm 98$ & RedFinal & & 1 \\
\hline f08-26 & $12: 42: 38.36$ & $+02: 37: 55.0$ & 4.41 & -139.4 & 22.10 & 1.83 & $800 \pm 37$ & $800 \pm 37$ & RedFinal & & 1 \\
\hline f08-27 & $12: 42: 27.38$ & $+02: 38: 12.0$ & 6.40 & -118.6 & 22.22 & 1.57 & $1014 \pm 51$ & $1014 \pm 51$ & RedFinal & & 1 \\
\hline f08-28 & $12: 42: 24.39$ & $+02: 38: 18.8$ & 7.01 & -114.9 & 20.85 & 1.23 & $1030 \pm 25$ & $1035 \pm 57$ & BlueFinal & $2.2: 59$ & 1 \\
\hline f08-29 & $12: 42: 35.34$ & $+02: 38: 21.4$ & 4.65 & -128.7 & 22.43 & 1.39 & $1292 \pm 98$ & $1292 \pm 98$ & Blue & & 1 \\
\hline f08-31 & $12: 42: 38.96$ & $+02: 38: 36.8$ & 3.80 & -134.2 & 19.47 & 1.89 & $820 \pm 12$ & $820 \pm 12$ & RedFinal & & 1 \\
\hline f08-32 & $12: 42: 27.27$ & $+02: 38: 45.9$ & 6.17 & -113.9 & 20.62 & 1.23 & $949 \pm 28$ & $949 \pm 28$ & BlueFinal & & 1 \\
\hline f08-33 & $12: 42: 36.46$ & $+02: 38: 47.7$ & 4.16 & -126.4 & 21.98 & 1.29 & $884 \pm 35$ & $884 \pm 35$ & BlueFinal & & 1 \\
\hline f08-37 & $12: 42: 36.69$ & $+02: 39: 21.7$ & 3.80 & -120.1 & 20.69 & 1.67 & $1081 \pm 14$ & $1098 \pm 36$ & RedFinal & f07-18 & 0 \\
\hline f08-39 & $12: 42: 34.64$ & $+02: 39: 33.2$ & 4.17 & -114.2 & 21.39 & 1.11 & $881 \pm 44$ & $881 \pm 44$ & BlueFinal & & 1 \\
\hline f08-40 & $12: 42: 34.93$ & $+02: 39: 39.5$ & 4.06 & -113.3 & 22.01 & 1.20 & $1077 \pm 56$ & $1084 \pm 69$ & Blue & f07-21 & 0 \\
\hline f08-41 & $12: 42: 23.15$ & $+02: 39: 44.5$ & 6.84 & -102.9 & 21.65 & 0.98 & $1005 \pm 64$ & $1007 \pm 100$ & Blue & $2.1: 83, \mathrm{f} 10-08$ & 1 \\
\hline f08-42 & $12: 42: 38.45$ & $+02: 39: 51.5$ & 3.18 & -116.3 & 21.93 & 1.75 & $809 \pm 43$ & $865 \pm 70$ & RedFinal & f07-23 & 0 \\
\hline f08-44 & $12: 42: 30.23$ & $+02: 40: 01.6$ & 5.06 & -104.2 & 22.36 & 1.82 & $819 \pm 47$ & $843 \pm 57$ & RedFinal & f10-10 & 1 \\
\hline f08-45 & $12: 42: 34.08$ & $+02: 40: 06.7$ & 4.11 & -106.3 & 22.26 & 1.85 & $955 \pm 40$ & $955 \pm 40$ & RedFinal & & 1 \\
\hline f08-46 & $12: 42: 36.87$ & $+02: 40: 12.2$ & 3.42 & -108.1 & 22.26 & 1.09 & $1257 \pm 53$ & $1257 \pm 53$ & BlueFinal & & 1 \\
\hline f08-47 & $12: 42: 36.86$ & $+02: 40: 18.6$ & 3.39 & -106.4 & 21.92 & 1.77 & $866 \pm 32$ & $866 \pm 49$ & RedFinal & f07-28 & 0 \\
\hline f08-48 & $12: 42: 39.30$ & $+02: 40: 25.5$ & 2.77 & -107.7 & 21.78 & 1.04 & $694 \pm 52$ & $679 \pm 95$ & Blue & f07-29 & 0 \\
\hline f08-50 & $12: 42: 38.31$ & $+02: 40: 35.0$ & 2.97 & -103.3 & 22.25 & 1.29 & $1208 \pm 51$ & $1208 \pm 51$ & BlueFinal & & 1 \\
\hline f08-52 & $12: 42: 37.19$ & $+02: 40: 43.0$ & 3.21 & -99.9 & 21.41 & 1.69 & $641 \pm 25$ & $641 \pm 25$ & RedFinal & & 1 \\
\hline f08-53 & $12: 42: 37.41$ & $+02: 40: 50.4$ & 3.14 & -97.8 & 21.23 & 1.88 & $1065 \pm 19$ & $1065 \pm 19$ & RedFinal & & 1 \\
\hline f08-54 & $12: 42: 29.45$ & $+02: 40: 57.3$ & 5.11 & -93.5 & 22.23 & 1.88 & $833 \pm 39$ & $833 \pm 39$ & RedFinal & & 1 \\
\hline f08-56 & $12: 42: 36.07$ & $+02: 41: 07.1$ & 3.45 & -92.5 & 22.32 & 1.27 & $932 \pm 83$ & $954 \pm 139$ & Blue & f07-34 & 0 \\
\hline f08-58 & $12: 42: 27.68$ & $+02: 41: 17.6$ & 5.54 & -89.7 & 21.39 & 1.54 & $909 \pm 34$ & $943 \pm 42$ & BlueFinal & f10-23 & 1 \\
\hline f08-59 & $12: 42: 26.83$ & $+02: 41: 23.7$ & 5.76 & -88.7 & 21.63 & 1.76 & $1173 \pm 59$ & $1217 \pm 66$ & RedFinal & f10-24 & 1 \\
\hline f08-60 & $12: 42: 22.56$ & $+02: 41: 28.0$ & 6.82 & -88.3 & 20.65 & 1.06 & $904 \pm 52$ & $954 \pm 82$ & Blue & f10-25 & 1 \\
\hline f08-61 & $12: 42: 38.16$ & $+02: 41: 30.5$ & 2.93 & -85.3 & 21.62 & 1.14 & $806 \pm 41$ & $847 \pm 55$ & BlueFinal & f07-38 & 0 \\
\hline f08-62 & $12: 42: 37.24$ & $+02: 41: 36.8$ & 3.17 & -83.7 & 22.07 & 1.70 & $809 \pm 33$ & $812 \pm 55$ & RedFinal & f07-39 & 0 \\
\hline f08-65 & $12: 42: 38.41$ & $+02: 41: 52.1$ & 2.92 & -78.1 & 20.95 & 1.56 & $950 \pm 19$ & $950 \pm 19$ & RedFinal & & 1 \\
\hline f08-66 & $12: 42: 33.87$ & $+02: 41: 57.7$ & 4.06 & -80.1 & 21.76 & 1.17 & $748 \pm 102$ & $770 \pm 111$ & Blue & f07-43 & 0 \\
\hline f08-67 & $12: 42: 29.77$ & $+02: 42: 03.5$ & 5.08 & -81.0 & 22.11 & 1.90 & $803 \pm 55$ & $803 \pm 55$ & RedFinal & & 1 \\
\hline f09-02 & $12: 42: 26.23$ & $+02: 43: 57.8$ & 6.49 & -65.4 & 21.29 & 1.23 & $851 \pm 55$ & $861 \pm 78$ & Blue & $3.2: 20, \mathrm{f} 10-47$ & 1 \\
\hline f09-03 & $12: 42: 32.20$ & $+02: 44: 05.2$ & 5.24 & -57.4 & 21.05 & 1.06 & $669 \pm 43$ & $669 \pm 43$ & BlueFinal & 1 & \\
\hline f09-04 & $12: 42: 31.72$ & $+02: 44: 14.3$ & 5.42 & -56.7 & 21.40 & 1.56 & $680 \pm 40$ & $666 \pm 54$ & RedFinal & $3.1: 24$, f10-49 & 1 \\
\hline f09-07 & $12: 42: 30.85$ & $+02: 44: 41.4$ & 5.85 & -54.2 & 20.69 & 1.27 & $680 \pm 26$ & $685 \pm 34$ & BlueFinal & $3.1: 31$ & 1 \\
\hline f09-10 & $12: 42: 25.99$ & $+02: 45: 03.1$ & 7.06 & -57.6 & 21.80 & 1.95 & $1060 \pm 45$ & $1060 \pm 45$ & RedFinal & & 1 \\
\hline f09-12 & $12: 42: 25.55$ & $+02: 45: 16.1$ & 7.27 & -56.6 & 22.00 & 1.74 & $852 \pm 48$ & $923 \pm 60$ & RedFinal & f10-61 & 1 \\
\hline f09-14 & $12: 42: 28.14$ & $+02: 45: 26.6$ & 6.85 & -52.4 & 21.14 & 1.63 & $938 \pm 36$ & $938 \pm 36$ & RedFinal & & 1 \\
\hline f09-21 & $12: 42: 19.95$ & $+02: 46: 20.5$ & 9.03 & -55.8 & 21.20 & 1.40 & $987 \pm 44$ & $987 \pm 44$ & BlueFinal & & 1 \\
\hline f09-25 & $12: 42: 23.50$ & $+02: 47: 03.9$ & 8.77 & -48.6 & 21.61 & 1.90 & $1041 \pm 30$ & $1041 \pm 30$ & RedFinal & & 1 \\
\hline f09-26 & $12: 42: 27.51$ & $+02: 47: 14.2$ & 8.17 & -43.1 & 22.49 & 1.43 & $852 \pm 71$ & $771 \pm 97$ & Blue & f13-18 & 1 \\
\hline f09-31 & $12: 42: 30.86$ & $+02: 47: 41.4$ & 7.99 & -36.5 & 19.89 & 1.54 & $1015 \pm 19$ & $1015 \pm 19$ & BlueFinal & & 1 \\
\hline f09-50 & $12: 42: 24.78$ & $+02: 49: 27.9$ & 10.32 & -37.4 & 21.40 & 1.17 & $1133 \pm 59$ & $1133 \pm 59$ & BlueFinal & & 1 \\
\hline
\end{tabular}


Y. Schuberth et al.: Dynamics of the NGC 4636 globular cluster system. II.

Table C.1. continued.

\begin{tabular}{|c|c|c|c|c|c|c|c|c|c|c|c|}
\hline ID & $\begin{array}{c}\mathrm{RA} \\
(\mathrm{J} 2000)\end{array}$ & $\begin{array}{c}\text { Dec } \\
(\mathrm{J} 2000)\end{array}$ & $\begin{array}{c}R_{4636} \\
{[\operatorname{arcmin}]}\end{array}$ & $\begin{array}{c}\text { PA } \\
{[\operatorname{deg}]}\end{array}$ & $\begin{array}{c}m_{R} \\
{[\mathrm{mag}]}\end{array}$ & $\begin{array}{l}C-R \\
{[\mathrm{mag}]}\end{array}$ & $\begin{array}{c}v_{\mathrm{spec}} \\
{\left[\mathrm{km} \mathrm{s}^{-1}\right]}\end{array}$ & $\begin{array}{c}v_{\text {final }} \\
{\left[\mathrm{km} \mathrm{s}^{-1}\right]}\end{array}$ & Sample & Cross-Identification(s) & $1 \mathrm{st}$ \\
\hline f09-58 & $12: 42: 24.46$ & $+02: 50: 12.1$ & 10.96 & -35.4 & 22.09 & 1.47 & $1046 \pm 110$ & $1046 \pm 110$ & Blue & & 1 \\
\hline f09-59 & $12: 42: 29.77$ & $+02: 50: 20.2$ & 10.37 & -29.0 & 21.90 & 1.61 & $694 \pm 66$ & $659 \pm 81$ & RedFinal & f13-47 & 1 \\
\hline f09-60 & $12: 42: 20.31$ & $+02: 50: 27.4$ & 11.79 & -38.8 & 20.96 & 1.26 & $920 \pm 44$ & $920 \pm 44$ & BlueFinal & & 1 \\
\hline f10-01 & $12: 42: 21.99$ & $+02: 39: 02.0$ & 7.31 & -107.8 & 21.14 & 1.20 & $842 \pm 35$ & $842 \pm 35$ & BlueFinal & & 1 \\
\hline f10-04 & $12: 42: 29.74$ & $+02: 39: 16.0$ & 5.41 & -111.7 & 22.56 & 1.26 & $877 \pm 73$ & $877 \pm 73$ & Blue & & 1 \\
\hline f10-08 & $12: 42: 23.15$ & $+02: 39: 44.5$ & 6.84 & -102.9 & 21.65 & 0.98 & $1061 \pm 67$ & $1007 \pm 100$ & Blue & $2.1: 83$, f08-41 & 0 \\
\hline f10-10 & $12: 42: 30.23$ & $+02: 40: 01.6$ & 5.06 & -104.2 & 22.36 & 1.82 & $867 \pm 32$ & $843 \pm 57$ & RedFinal & f08-44 & 0 \\
\hline f10-15 & $12: 42: 16.72$ & $+02: 40: 32.5$ & 8.31 & -95.0 & 22.09 & 0.94 & $1139 \pm 44$ & $1139 \pm 44$ & BlueFinal & & 1 \\
\hline f10-16 & $12: 42: 30.93$ & $+02: 40: 38.6$ & 4.77 & -97.5 & 21.81 & 1.81 & $947 \pm 36$ & $978 \pm 44$ & RedFinal & f07-31 & 0 \\
\hline f10-18 & $12: 42: 23.15$ & $+02: 40: 51.3$ & 6.69 & -93.5 & 21.82 & 1.83 & $811 \pm 36$ & $811 \pm 36$ & RedFinal & & 1 \\
\hline f10-23 & $12: 42: 27.68$ & $+02: 41: 17.6$ & 5.54 & -89.7 & 21.39 & 1.54 & $977 \pm 25$ & $943 \pm 42$ & BlueFinal & f08-58 & 0 \\
\hline f10-24 & $12: 42: 26.83$ & $+02: 41: 23.7$ & 5.76 & -88.7 & 21.63 & 1.76 & $1261 \pm 29$ & $1217 \pm 66$ & RedFinal & f08-59 & 0 \\
\hline f10-25 & $12: 42: 22.56$ & $+02: 41: 28.0$ & 6.82 & -88.3 & 20.65 & 1.06 & $1004 \pm 63$ & $954 \pm 82$ & Blue & f08-60 & 0 \\
\hline f10-35 & $12: 42: 20.66$ & $+02: 42: 44.6$ & 7.44 & -78.6 & 21.53 & 1.62 & $961 \pm 24$ & $961 \pm 24$ & RedFinal & & 1 \\
\hline f10-38 & $12: 42: 27.30$ & $+02: 43: 06.3$ & 5.93 & -71.9 & 22.15 & 1.05 & $1267 \pm 52$ & $1267 \pm 52$ & BlueFinal & & 1 \\
\hline f10-42 & $12: 42: 22.15$ & $+02: 43: 29.5$ & 7.27 & -72.2 & 21.73 & 1.77 & $900 \pm 34$ & $900 \pm 34$ & RedFinal & & 1 \\
\hline f10-43 & $12: 42: 22.17$ & $+02: 43: 30.9$ & 7.27 & -72.0 & 22.11 & 1.09 & $1044 \pm 58$ & $1044 \pm 58$ & BlueFinal & & 1 \\
\hline f10-44 & $12: 42: 30.46$ & $+02: 43: 40.4$ & 5.41 & -63.6 & 21.84 & 1.07 & $1185 \pm 56$ & $1165 \pm 79$ & Blue & f07-63 & 0 \\
\hline f10-45 & $12: 42: 28.85$ & $+02: 43: 49.8$ & 5.84 & -64.0 & 21.36 & 1.29 & $1054 \pm 23$ & $980 \pm 45$ & BlueFinal & $3.1: 17$ & 1 \\
\hline f10-47 & $12: 42: 26.23$ & $+02: 43: 57.8$ & 6.49 & -65.4 & 21.29 & 1.23 & $872 \pm 30$ & $861 \pm 78$ & Blue & $3.2: 20$, f09-02 & 0 \\
\hline f10-49 & $12: 42: 31.72$ & $+02: 44: 14.3$ & 5.42 & -56.7 & 21.40 & 1.56 & $798 \pm 23$ & $666 \pm 54$ & RedFinal & $3.1: 24$, f09-04 & 0 \\
\hline f10-57 & $12: 42: 27.33$ & $+02: 44: 52.6$ & 6.69 & -57.3 & 20.69 & 1.31 & $860 \pm 62$ & $798 \pm 65$ & Blue & $3.2: 37$ & 1 \\
\hline f10-58 & $12: 42: 23.52$ & $+02: 44: 56.7$ & 7.54 & -60.8 & 21.73 & 1.25 & $1226 \pm 56$ & $1226 \pm 56$ & BlueFinal & & 1 \\
\hline f10-61 & $12: 42: 25.55$ & $+02: 45: 16.1$ & 7.27 & -56.6 & 22.00 & 1.74 & $993 \pm 35$ & $923 \pm 60$ & RedFinal & f09-12 & 0 \\
\hline f10-63 & $12: 42: 27.65$ & $+02: 45: 25.5$ & 6.93 & -53.1 & 19.99 & 1.50 & $1167 \pm 11$ & $1167 \pm 11$ & BlueFinal & & 1 \\
\hline f10-65 & $12: 42: 14.52$ & $+02: 45: 32.5$ & 9.81 & -64.2 & 22.51 & 1.73 & $1023 \pm 49$ & $1023 \pm 49$ & RedFinal & & 1 \\
\hline f10-68 & $12: 42: 24.13$ & $+02: 45: 56.5$ & 7.95 & -54.0 & 22.68 & 1.76 & $1167 \pm 38$ & $1167 \pm 38$ & RedFinal & & 1 \\
\hline f12-08 & $12: 43: 08.14$ & $+02: 37: 52.3$ & 5.69 & 126.7 & 22.34 & 1.75 & $1100 \pm 36$ & $1100 \pm 36$ & RedFinal & & 1 \\
\hline f12-11 & $12: 43: 10.67$ & $+02: 38: 11.1$ & 6.04 & 120.7 & 21.86 & 1.75 & $729 \pm 23$ & $754 \pm 36$ & RedFinal & f01-43 & 0 \\
\hline f $12-12$ & $12: 43: 06.38$ & $+02: 38: 22.5$ & 5.04 & 125.0 & 22.10 & 1.11 & $824 \pm 58$ & $789 \pm 82$ & Blue & f01-45 & 0 \\
\hline f12-13 & $12: 43: 22.26$ & $+02: 38: 26.6$ & 8.57 & 109.2 & 20.82 & 1.23 & $1087 \pm 29$ & $1087 \pm 29$ & BlueFinal & & 1 \\
\hline f12-15 & $12: 43: 21.12$ & $+02: 38: 39.1$ & 8.23 & 108.5 & 21.86 & 1.76 & $956 \pm 27$ & $956 \pm 27$ & RedFinal & & 1 \\
\hline f12-17 & $12: 43: 15.37$ & $+02: 38: 52.4$ & 6.80 & 110.6 & 20.18 & 1.13 & $1067 \pm 34$ & $1067 \pm 34$ & BlueFinal & & 1 \\
\hline f12-18 & $12: 43: 12.92$ & $+02: 39: 01.3$ & 6.18 & 111.3 & 22.38 & 1.46 & $869 \pm 90$ & $869 \pm 90$ & Blue & & 1 \\
\hline f12-19 & $12: 43: 08.76$ & $+02: 39: 11.0$ & 5.16 & 113.8 & 19.85 & 1.22 & $1038 \pm 21$ & $1043 \pm 30$ & BlueFinal & $6.1: 45$ & 1 \\
\hline f12-23 & $12: 43: 17.10$ & $+02: 39: 24.9$ & 7.05 & 105.2 & 21.32 & 1.26 & $1387 \pm 44$ & $1348 \pm 76$ & Blue & $6.2: 57$ & 1 \\
\hline f12-24 & $12: 43: 09.28$ & $+02: 39: 33.2$ & 5.14 & 109.5 & 19.85 & 1.62 & $968 \pm 2$ & $979 \pm 15$ & RedFinal & $6.2: 59$ & 1 \\
\hline f12-25 & $12: 43: 11.71$ & $+02: 39: 36.4$ & 5.70 & 106.9 & 22.32 & 1.37 & $902 \pm 53$ & $902 \pm 53$ & BlueFinal & & 1 \\
\hline f12-26 & $12: 43: 09.97$ & $+02: 39: 43.2$ & 5.25 & 107.1 & 22.04 & 1.20 & $875 \pm 56$ & $875 \pm 56$ & BlueFinal & & 1 \\
\hline f12-27 & $12: 43: 16.86$ & $+02: 39: 51.8$ & 6.88 & 101.8 & 21.27 & 1.34 & $705 \pm 26$ & $733 \pm 50$ & BlueFinal & $6.1: 57$ & 1 \\
\hline f12-28 & $12: 43: 12.83$ & $+02: 40: 00.7$ & 5.87 & 102.3 & 21.55 & 1.23 & $895 \pm 45$ & $895 \pm 45$ & BlueFinal & & 1 \\
\hline f12-29 & $12: 43: 09.65$ & $+02: 40: 05.0$ & 5.08 & 103.5 & 22.17 & 1.99 & $682 \pm 25$ & $682 \pm 25$ & RedFinal & & 1 \\
\hline f12-30 & $12: 43: 11.63$ & $+02: 40: 28.3$ & 5.49 & 98.3 & 22.04 & 1.31 & $825 \pm 32$ & $825 \pm 32$ & BlueFinal & & 1 \\
\hline f12-33 & $12: 43: 05.93$ & $+02: 40: 45.5$ & 4.04 & 97.2 & 20.80 & 1.21 & $685 \pm 25$ & $645 \pm 33$ & BlueFinal & f02-35 & 0 \\
\hline f12-34 & $12: 43: 12.80$ & $+02: 40: 51.6$ & 5.74 & 94.1 & 22.25 & 1.49 & $1036 \pm 35$ & $1036 \pm 35$ & BlueFinal & & 1 \\
\hline f12-36 & $12: 43: 08.06$ & $+02: 41: 08.8$ & 4.54 & 91.5 & 21.45 & 1.41 & $821 \pm 30$ & $821 \pm 30$ & BlueFinal & & 1 \\
\hline f12-38 & $12: 43: 06.92$ & $+02: 41: 25.6$ & 4.26 & 87.8 & 21.13 & 1.67 & $867 \pm 15$ & $854 \pm 37$ & RedFinal & f02-42 & 0 \\
\hline f12-42 & $12: 43: 14.68$ & $+02: 41: 53.6$ & 6.23 & 84.2 & 21.23 & 2.08 & $630 \pm 21$ & $622 \pm 35$ & RedFinal & $6.2: 95$ & 1 \\
\hline f12-44 & 12:43:09.04 & $+02: 42: 06.1$ & 4.86 & 80.1 & 20.02 & 1.54 & $745 \pm 14$ & $713 \pm 30$ & BlueFinal & $6.1: 92$, f02-48 & 0 \\
\hline f12-48 & $12: 43: 12.33$ & $+02: 42: 34.2$ & 5.76 & 76.9 & 22.27 & 1.26 & $1042 \pm 67$ & $1042 \pm 67$ & Blue & & 1 \\
\hline f $12-50$ & $12: 43: 22.16$ & $+02: 42: 50.5$ & 8.22 & 78.9 & 21.98 & 1.73 & $709 \pm 27$ & $709 \pm 27$ & RedFinal & & 1 \\
\hline f12-51 & $12: 43: 08.47$ & $+02: 42: 57.9$ & 4.95 & 69.9 & 21.16 & 1.15 & $1043 \pm 31$ & $1011 \pm 44$ & BlueFinal & f02-58 & 0 \\
\hline f12-53 & $12: 43: 10.45$ & $+02: 43: 14.0$ & 5.50 & 69.1 & 22.32 & 1.76 & $780 \pm 35$ & $730 \pm 61$ & RedFinal & f02-60 & 0 \\
\hline f12-58 & $12: 43: 06.36$ & $+02: 43: 45.6$ & 4.81 & 58.8 & 21.70 & 1.77 & $1022 \pm 38$ & $952 \pm 67$ & RedFinal & f02-65, f03-02 & 0 \\
\hline f12-62 & $12: 43: 14.73$ & $+02: 44: 04.8$ & 6.82 & 65.6 & 20.57 & 1.72 & $874 \pm 20$ & $874 \pm 20$ & RedFinal & & 1 \\
\hline f13-06 & $12: 42: 38.70$ & $+02: 45: 58.3$ & 5.47 & -30.7 & 21.70 & 1.58 & $674 \pm 23$ & $661 \pm 46$ & RedFinal & $3.1: 50$ & 1 \\
\hline f13-08 & $12: 42: 39.14$ & $+02: 46: 10.1$ & 5.59 & -28.7 & 22.37 & 1.57 & $1246 \pm 36$ & $1246 \pm 36$ & RedFinal & & 1 \\
\hline f13-10 & $12: 42: 23.31$ & $+02: 46: 21.6$ & 8.36 & -52.5 & 21.85 & 1.67 & $700 \pm 53$ & $700 \pm 53$ & RedFinal & & 1 \\
\hline f13-11 & $12: 42: 39.94$ & $+02: 46: 33.7$ & 5.85 & -25.1 & 22.29 & 1.52 & $1064 \pm 41$ & $1064 \pm 41$ & BlueFinal & & 1 \\
\hline f13-12 & $12: 42: 32.57$ & $+02: 46: 38.4$ & 6.89 & -38.8 & 21.39 & 1.21 & $508 \pm 75$ & $508 \pm 75$ & Blue & & 1 \\
\hline f13-13 & $12: 42: 42.06$ & $+02: 46: 48.9$ & 5.88 & -19.4 & 20.24 & 1.61 & $978 \pm 13$ & $978 \pm 27$ & RedFinal & f05-61, f04-11 & 0 \\
\hline f13-15 & $12: 42: 34.82$ & $+02: 46: 59.5$ & 6.85 & -33.3 & 20.46 & 1.20 & $1432 \pm 28$ & $1428 \pm 38$ & Blue & $3.1: 69$ & 1 \\
\hline f13-17 & $12: 42: 37.11$ & $+02: 47: 09.5$ & 6.70 & -28.4 & 22.11 & 1.19 & $875 \pm 68$ & $875 \pm 68$ & Blue & & 1 \\
\hline f13-18 & $12: 42: 27.51$ & $+02: 47: 14.2$ & 8.17 & -43.1 & 22.49 & 1.43 & $690 \pm 66$ & $771 \pm 97$ & Blue & f09-26 & 0 \\
\hline f13-23 & $12: 42: 43.07$ & $+02: 47: 39.9$ & 6.62 & -14.9 & 22.06 & 1.47 & $918 \pm 38$ & $918 \pm 38$ & BlueFinal & & 1 \\
\hline f13-24 & $12: 42: 38.86$ & $+02: 47: 48.1$ & 7.09 & -22.8 & 22.11 & 1.28 & $662 \pm 39$ & $644 \pm 91$ & Blue & f04-20 & 0 \\
\hline
\end{tabular}


Table C.1. continued.

\begin{tabular}{|c|c|c|c|c|c|c|c|c|c|c|c|}
\hline ID & $\begin{array}{c}\text { RA } \\
(\mathrm{J} 2000)\end{array}$ & $\begin{array}{c}\text { Dec } \\
(\mathrm{J} 2000)\end{array}$ & $\begin{array}{c}R_{4636} \\
{[\operatorname{arcmin}]}\end{array}$ & $\begin{array}{c}\text { PA } \\
{[\mathrm{deg}]}\end{array}$ & $\begin{array}{c}m_{R} \\
{[\mathrm{mag}]}\end{array}$ & $\begin{array}{l}C-R \\
{[\mathrm{mag}]}\end{array}$ & $\begin{array}{c}v_{\mathrm{spec}} \\
{\left[\mathrm{km} \mathrm{s}^{-1}\right]}\end{array}$ & $\begin{array}{c}v_{\text {final }} \\
{\left[\mathrm{km} \mathrm{s}^{-1}\right]}\end{array}$ & Sample & Cross-Identification(s) & $1 \mathrm{st}$ \\
\hline f13-25 & $12: 42: 41.06$ & $+02: 47: 52.2$ & 6.96 & -18.4 & 21.90 & 1.63 & $623 \pm 37$ & $595 \pm 86$ & RedFinal & f04-21 & 0 \\
\hline f13-31 & $12: 42: 34.70$ & $+02: 48: 29.3$ & 8.15 & -27.7 & 22.16 & 1.46 & $1427 \pm 99$ & $1427 \pm 99$ & Blue & & 1 \\
\hline f13-32 & $12: 42: 25.39$ & $+02: 48: 44.7$ & 9.66 & -39.3 & 21.80 & 1.68 & $1092 \pm 34$ & $1092 \pm 34$ & RedFinal & & 1 \\
\hline f13-41 & $12: 42: 39.58$ & $+02: 49: 44.8$ & 8.86 & -16.9 & 22.05 & 1.13 & $972 \pm 60$ & $972 \pm 60$ & BlueFinal & & 1 \\
\hline f13-44 & $12: 42: 26.61$ & $+02: 49: 58.8$ & 10.47 & -33.7 & 22.20 & 1.65 & $858 \pm 37$ & $858 \pm 37$ & RedFinal & & 1 \\
\hline f13-45 & $12: 42: 33.37$ & $+02: 50: 04.6$ & 9.73 & -25.1 & 22.45 & 1.81 & $1079 \pm 27$ & $1079 \pm 27$ & RedFinal & & 1 \\
\hline f13-47 & $12: 42: 29.77$ & $+02: 50: 20.2$ & 10.37 & -29.0 & 21.90 & 1.61 & $624 \pm 47$ & $659 \pm 81$ & RedFinal & f09-59 & 0 \\
\hline f13-50 & $12: 42: 32.37$ & $+02: 50: 37.6$ & 10.33 & -25.0 & 22.36 & 2.04 & $833 \pm 34$ & $833 \pm 34$ & RedFinal & & 1 \\
\hline f13-54 & $12: 42: 36.67$ & $+02: 51: 12.5$ & 10.47 & -18.3 & 20.99 & 1.76 & $1034 \pm 17$ & $1034 \pm 17$ & RedFinal & & 1 \\
\hline f13-56 & $12: 42: 37.51$ & $+02: 51: 28.7$ & 10.67 & -16.8 & 22.01 & 1.62 & $1074 \pm 36$ & $1074 \pm 36$ & RedFinal & & 1 \\
\hline f13-61 & $12: 42: 29.38$ & $+02: 52: 00.0$ & 11.89 & -25.5 & 21.93 & 1.84 & $788 \pm 39$ & $774 \pm 75$ & RedFinal & $4.1: 33$ & 1 \\
\hline f13-64 & $12: 42: 37.76$ & $+02: 52: 13.6$ & 11.37 & -15.4 & 22.16 & 1.70 & $848 \pm 58$ & $848 \pm 58$ & RedFinal & & 1 \\
\hline $1.1: 7$ & $12: 42: 52.05$ & $+02: 36: 18.8$ & 4.98 & 173.7 & 21.22 & 1.83 & $1032 \pm 22$ & $1032 \pm 22$ & RedFinal & & 1 \\
\hline $1.1: 8$ & $12: 42: 49.92$ & $+02: 36: 23.4$ & 4.88 & 179.9 & 21.20 & 1.80 & $682 \pm 42$ & $682 \pm 42$ & RedFinal & & 1 \\
\hline $1.1: 9$ & $12: 42: 45.17$ & $+02: 36: 27.6$ & 4.95 & -166.3 & 20.91 & 1.34 & $663 \pm 30$ & $663 \pm 30$ & BlueFinal & & 1 \\
\hline $1.1: 16$ & $12: 42: 53.54$ & $+02: 36: 55.2$ & 4.44 & 168.1 & 21.29 & 1.76 & $968 \pm 30$ & $968 \pm 30$ & RedFinal & & 1 \\
\hline $1.1: 27$ & $12: 42: 45.50$ & $+02: 37: 36.7$ & 3.81 & -163.4 & 21.31 & 1.86 & $682 \pm 22$ & $682 \pm 22$ & RedFinal & & 1 \\
\hline $1.1: 28$ & $12: 42: 54.08$ & $+02: 37: 41.9$ & 3.72 & 163.6 & 21.03 & 1.19 & $968 \pm 45$ & $968 \pm 45$ & BlueFinal & & 1 \\
\hline $1.1: 32$ & $12: 42: 44.81$ & $+02: 37: 58.1$ & 3.53 & -159.0 & 19.93 & 1.56 & $852 \pm 23$ & $868 \pm 27$ & RedFinal & f07-02 & 0 \\
\hline $1.1: 33$ & $12: 42: 49.79$ & $+02: 38: 00.4$ & 3.26 & -179.6 & 21.41 & 1.44 & $880 \pm 32$ & $880 \pm 32$ & BlueFinal & & 1 \\
\hline $1.1: 34$ & $12: 42: 56.55$ & $+02: 38: 04.9$ & 3.60 & 152.4 & 20.57 & 1.12 & $895 \pm 45$ & $881 \pm 54$ & BlueFinal & f02-09 & 0 \\
\hline $1.1: 36$ & $12: 42: 44.36$ & $+02: 38: 13.0$ & 3.35 & -155.7 & 20.16 & 1.45 & $666 \pm 24$ & $676 \pm 27$ & BlueFinal & f07-05 & 0 \\
\hline $1.1: 39$ & $12: 42: 53.93$ & $+02: 38: 25.9$ & 3.01 & 160.3 & 21.50 & 1.33 & $991 \pm 36$ & $991 \pm 36$ & BlueFinal & & 1 \\
\hline $1.1: 40$ & $12: 42: 43.41$ & $+02: 38: 29.7$ & 3.21 & -149.8 & 21.06 & 1.38 & $586 \pm 26$ & $637 \pm 34$ & BlueFinal & f07-08 & 0 \\
\hline $1.1: 44$ & $12: 42: 53.28$ & $+02: 38: 44.0$ & 2.67 & 161.4 & 19.64 & 1.18 & $1210 \pm 25$ & $1260 \pm 35$ & BlueFinal & f06-68 & 0 \\
\hline $1.1: 46$ & $12: 42: 48.19$ & $+02: 39: 00.7$ & 2.29 & -169.5 & 20.23 & 1.46 & $890 \pm 18$ & $890 \pm 18$ & $\mathrm{R} 2.5$ & & 1 \\
\hline $1.1: 47$ & $12: 42: 44.72$ & $+02: 39: 05.1$ & 2.53 & -149.5 & 20.30 & 1.85 & $1092 \pm 21$ & $1115 \pm 25$ & RedFinal & f07-15 & 0 \\
\hline $1.1: 52$ & $12: 42: 49.91$ & $+02: 39: 22.1$ & 1.90 & 179.7 & 20.40 & 1.59 & $1217 \pm 20$ & $1217 \pm 20$ & $\mathrm{R} 2.5$ & & 1 \\
\hline $1.1: 53$ & $12: 42: 44.17$ & $+02: 39: 27.1$ & 2.31 & -141.9 & 19.80 & 1.33 & $1210 \pm 18$ & $1219 \pm 23$ & R2.5 & f07-19 & 0 \\
\hline $1.1: 56$ & $12: 42: 42.20$ & $+02: 39: 36.7$ & 2.53 & -130.8 & 19.74 & 1.64 & $1112 \pm 17$ & $1112 \pm 17$ & RedFinal & & 1 \\
\hline $1.1: 62$ & $12: 42: 47.53$ & $+02: 40: 00.4$ & 1.39 & -155.1 & 20.87 & 1.45 & $764 \pm 17$ & $764 \pm 17$ & $\mathrm{R} 2.5$ & & 1 \\
\hline $1.1: 63$ & $12: 42: 52.33$ & $+02: 40: 04.3$ & 1.34 & 152.8 & 19.11 & 1.62 & $725 \pm 17$ & $725 \pm 17$ & $\mathrm{R} 2.5$ & & 1 \\
\hline $1.1: 71$ & $12: 42: 42.58$ & $+02: 40: 32.0$ & 1.96 & -111.9 & 20.06 & 1.55 & $902 \pm 14$ & $918 \pm 21$ & $\mathrm{R} 2.5$ & f07-30 & 0 \\
\hline $1.1: 87$ & $12: 42: 44.81$ & $+02: 41: 24.1$ & 1.27 & -83.9 & 20.32 & 1.55 & $502 \pm 17$ & $502 \pm 17$ & $\mathrm{R} 2.5$ & & 1 \\
\hline $1.1: 91$ & $12: 42: 53.62$ & $+02: 41: 38.2$ & 1.01 & 68.4 & 20.22 & 1.62 & $1051 \pm 20$ & $1051 \pm 20$ & R2.5 & & 1 \\
\hline $1.1: 93$ & $12: 42: 43.16$ & $+02: 41: 46.6$ & 1.75 & -73.1 & 20.52 & 1.45 & $991 \pm 19$ & $991 \pm 19$ & $\mathrm{R} 2.5$ & & 1 \\
\hline $1.1: 102$ & $12: 42: 42.78$ & $+02: 42: 17.5$ & 2.05 & -59.9 & 20.39 & 1.83 & $1252 \pm 19$ & $1252 \pm 19$ & $\mathrm{R} 2.5$ & & 1 \\
\hline $1.1: 103$ & $12: 42: 49.89$ & $+02: 42: 20.3$ & 1.07 & 0.3 & 20.76 & 1.18 & $968 \pm 31$ & $968 \pm 31$ & $\mathrm{R} 2.5$ & & 1 \\
\hline $1.1: 106$ & $12: 42: 46.82$ & $+02: 42: 32.4$ & 1.48 & -30.9 & 20.90 & 1.06 & $530 \pm 43$ & $530 \pm 43$ & R2.5 & & 1 \\
\hline $1.2: 1$ & $12: 42: 43.99$ & $+02: 35: 52.0$ & 5.60 & -164.8 & 21.05 & 1.20 & $1230 \pm 64$ & $1230 \pm 64$ & BlueFinal & & 1 \\
\hline $1.2: 2$ & $12: 42: 42.47$ & $+02: 35: 55.8$ & 5.65 & -160.9 & 22.13 & 1.16 & $1040 \pm 60$ & $1040 \pm 60$ & BlueFinal & & 1 \\
\hline $1.2: 6$ & $12: 42: 45.91$ & $+02: 36: 14.1$ & 5.13 & -168.9 & 20.66 & 1.46 & $860 \pm 17$ & $860 \pm 17$ & BlueFinal & & 1 \\
\hline $1.2: 9$ & $12: 42: 42.55$ & $+02: 36: 23.7$ & 5.20 & -159.4 & 21.81 & 1.55 & $1198 \pm 52$ & $1198 \pm 52$ & BlueFinal & & 1 \\
\hline $1.2: 11$ & $12: 42: 44.49$ & $+02: 36: 31.7$ & 4.93 & -164.2 & 22.24 & 1.19 & $888 \pm 86$ & $888 \pm 86$ & Blue & & 1 \\
\hline $1.2: 15$ & $12: 42: 41.23$ & $+02: 36: 51.1$ & 4.91 & -154.0 & 21.61 & 1.70 & $392 \pm 38$ & $392 \pm 38$ & Red & & 1 \\
\hline $1.2: 18$ & $12: 42: 52.63$ & $+02: 37: 05.3$ & 4.23 & 170.6 & 22.27 & 1.78 & $787 \pm 29$ & $787 \pm 29$ & RedFinal & & 1 \\
\hline $1.2: 20$ & $12: 42: 42.09$ & $+02: 37: 14.4$ & 4.47 & -154.2 & 21.77 & 1.67 & $904 \pm 33$ & $904 \pm 33$ & RedFinal & & 1 \\
\hline $1.2: 23$ & $12: 42: 47.62$ & $+02: 37: 22.6$ & 3.93 & -171.8 & 20.15 & 1.50 & $1112 \pm 15$ & $1086 \pm 37$ & BlueFinal & $1.3: 25$ & 1 \\
\hline $1.2: 25$ & $12: 42: 51.66$ & $+02: 37: 30.7$ & 3.78 & 173.2 & 20.83 & 1.81 & $746 \pm 31$ & $694 \pm 37$ & RedFinal & $1.3: 27$ & 1 \\
\hline $1.2: 27$ & $12: 42: 42.78$ & $+02: 37: 36.5$ & 4.06 & -154.2 & 20.73 & 1.16 & $730 \pm 62$ & $730 \pm 62$ & BlueFinal & & 1 \\
\hline $1.2: 33$ & $12: 42: 47.94$ & $+02: 38: 02.3$ & 3.26 & -171.5 & 22.62 & 1.91 & $909 \pm 32$ & $909 \pm 32$ & RedFinal & & 1 \\
\hline $1.2: 35$ & $12: 42: 50.71$ & $+02: 38: 10.3$ & 3.10 & 176.1 & 21.67 & 1.37 & $1121 \pm 42$ & $1121 \pm 42$ & BlueFinal & & 1 \\
\hline $1.2: 36$ & $12: 42: 49.50$ & $+02: 38: 14.4$ & 3.03 & -178.3 & 21.69 & 1.96 & $840 \pm 43$ & $840 \pm 43$ & RedFinal & & 1 \\
\hline $1.2: 38$ & $12: 42: 43.37$ & $+02: 38: 22.2$ & 3.32 & -150.7 & 21.67 & 1.67 & $870 \pm 30$ & $870 \pm 30$ & RedFinal & & 1 \\
\hline $1.2: 40$ & $12: 42: 44.71$ & $+02: 38: 30.3$ & 3.05 & -155.0 & 21.48 & 1.58 & $816 \pm 26$ & $816 \pm 26$ & RedFinal & & 1 \\
\hline $1.2: 41$ & $12: 42: 51.23$ & $+02: 38: 33.8$ & 2.72 & 172.8 & 21.51 & 1.71 & $626 \pm 22$ & $626 \pm 22$ & RedFinal & & 1 \\
\hline $1.2: 42$ & $12: 42: 42.28$ & $+02: 38: 36.7$ & 3.26 & -144.5 & 22.12 & 1.74 & $1040 \pm 43$ & $1040 \pm 43$ & RedFinal & & 1 \\
\hline $1.2: 49$ & $12: 42: 52.19$ & $+02: 39: 26.0$ & 1.92 & 162.5 & 22.34 & 1.54 & $901 \pm 28$ & $901 \pm 28$ & $\mathrm{R} 2.5$ & & 1 \\
\hline $1.2: 55$ & $12: 42: 51.61$ & $+02: 39: 46.5$ & 1.55 & 163.8 & 20.34 & 1.21 & $718 \pm 21$ & $718 \pm 21$ & R2.5 & & 1 \\
\hline $1.2: 57$ & $12: 42: 45.51$ & $+02: 39: 53.2$ & 1.76 & -141.7 & 19.50 & 1.64 & $682 \pm 15$ & $682 \pm 15$ & R2.5 & & 1 \\
\hline $1.2: 58$ & $12: 42: 45.67$ & $+02: 39: 57.0$ & 1.68 & -141.5 & 19.88 & 1.36 & $670 \pm 14$ & $670 \pm 14$ & $\mathrm{R} 2.5$ & & 1 \\
\hline $1.2: 61$ & $12: 42: 40.47$ & $+02: 40: 09.1$ & 2.60 & -115.4 & 21.68 & 1.30 & $832 \pm 26$ & $832 \pm 26$ & BlueFinal & & 1 \\
\hline $1.2: 62$ & $12: 42: 46.55$ & $+02: 40: 12.8$ & 1.34 & -141.8 & 20.78 & 1.19 & $848 \pm 49$ & $848 \pm 49$ & $\mathrm{R} 2.5$ & & 1 \\
\hline $1.2: 65$ & $12: 42: 43.55$ & $+02: 40: 29.6$ & 1.76 & -116.1 & 21.45 & 1.52 & $858 \pm 31$ & $858 \pm 31$ & $\mathrm{R} 2.5$ & & 1 \\
\hline $1.2: 67$ & $12: 42: 44.83$ & $+02: 40: 42.4$ & 1.38 & -114.0 & 20.69 & 1.61 & $720 \pm 33$ & $720 \pm 33$ & R2.5 & & 1 \\
\hline $1.2: 69$ & $12: 42: 42.83$ & $+02: 40: 47.1$ & 1.82 & -105.3 & 21.87 & 1.60 & $1159 \pm 22$ & $1159 \pm 22$ & $\mathrm{R} 2.5$ & & 1 \\
\hline
\end{tabular}


Y. Schuberth et al.: Dynamics of the NGC 4636 globular cluster system. II.

Table C.1. continued.

\begin{tabular}{|c|c|c|c|c|c|c|c|c|c|c|c|}
\hline ID & $\begin{array}{c}\mathrm{RA} \\
(\mathrm{J} 2000)\end{array}$ & $\begin{array}{c}\text { Dec } \\
(\mathrm{J} 2000)\end{array}$ & $\begin{array}{c}R_{4636} \\
{[\operatorname{arcmin}]}\end{array}$ & $\begin{array}{c}\text { PA } \\
{[\mathrm{deg}]}\end{array}$ & $\begin{array}{c}m_{R} \\
{[\mathrm{mag}]}\end{array}$ & $\begin{array}{l}C-R \\
{[\mathrm{mag}]}\end{array}$ & $\begin{array}{c}v_{\text {spec }} \\
{\left[\mathrm{km} \mathrm{s}^{-1}\right]}\end{array}$ & $\begin{array}{c}v_{\text {final }} \\
{\left[\mathrm{km} \mathrm{s}^{-1}\right]}\end{array}$ & Sample & Cross-Identification(s) & $1 \mathrm{st}$ \\
\hline $1.2: 71$ & $12: 42: 41.09$ & $+02: 40: 56.7$ & 2.22 & -98.3 & 21.92 & 1.59 & $1010 \pm 28$ & $1010 \pm 28$ & $\mathrm{R} 2.5$ & & 1 \\
\hline $1.2: 72$ & $12: 42: 41.98$ & $+02: 41: 00.8$ & 1.99 & -97.3 & 20.01 & 1.60 & $607 \pm 17$ & $607 \pm 17$ & R2.5 & & 1 \\
\hline $1.2: 75$ & $12: 42: 42.35$ & $+02: 41: 15.1$ & 1.88 & -90.5 & 19.22 & 1.63 & $1027 \pm 16$ & $1059 \pm 29$ & $\mathrm{R} 2.5$ & f07-35 & 0 \\
\hline $1.2: 76$ & $12: 42: 43.71$ & $+02: 41: 19.0$ & 1.54 & -88.1 & 20.80 & 0.98 & $1187 \pm 36$ & $1182 \pm 48$ & $\mathrm{R} 2.5$ & f07-36 & 0 \\
\hline $1.2: 77$ & $12: 42: 42.00$ & $+02: 41: 22.9$ & 1.97 & -86.7 & 20.82 & 1.31 & $958 \pm 23$ & $958 \pm 23$ & R2.5 & & 1 \\
\hline $1.2: 78$ & $12: 42: 42.40$ & $+02: 41: 26.3$ & 1.87 & -84.7 & 20.75 & 1.70 & $933 \pm 17$ & $933 \pm 17$ & R2.5 & & 1 \\
\hline $1.2: 83$ & $12: 42: 43.79$ & $+02: 41: 50.1$ & 1.62 & -69.5 & 21.13 & 1.61 & $460 \pm 30$ & $456 \pm 36$ & R2.5 & f05-17 & 0 \\
\hline $1.2: 85$ & $12: 42: 43.64$ & $+02: 41: 59.8$ & 1.72 & -64.9 & 21.13 & 1.11 & $1070 \pm 27$ & $1070 \pm 27$ & R2.5 & & 1 \\
\hline $1.2: 86$ & $12: 42: 47.40$ & $+02: 42: 05.1$ & 1.02 & -37.0 & 20.21 & 1.60 & $1159 \pm 14$ & $1159 \pm 14$ & R2.5 & & 1 \\
\hline $1.2: 88$ & $12: 42: 50.28$ & $+02: 42: 11.4$ & 0.93 & 6.3 & 20.77 & 1.34 & $971 \pm 18$ & $971 \pm 18$ & R2.5 & & 1 \\
\hline $1.2: 91$ & $12: 42: 42.48$ & $+02: 42: 21.3$ & 2.14 & -59.5 & 20.22 & 1.50 & $810 \pm 14$ & $810 \pm 14$ & $\mathrm{R} 2.5$ & & 1 \\
\hline $1.2: 93$ & $12: 42: 45.67$ & $+02: 42: 28.0$ & 1.59 & -41.2 & 19.27 & 1.39 & $1002 \pm 17$ & $1002 \pm 17$ & R2.5 & & 1 \\
\hline $1.2: 95$ & $12: 42: 41.65$ & $+02: 42: 34.3$ & 2.43 & -57.6 & 21.25 & 1.08 & $943 \pm 42$ & $943 \pm 42$ & R2.5 & & 1 \\
\hline $1.3: 3$ & $12: 42: 49.71$ & $+02: 36: 02.9$ & 5.22 & -179.6 & 20.70 & 1.84 & $653 \pm 29$ & $693 \pm 37$ & RedFinal & f06-38 & 0 \\
\hline $1.3: 19$ & $12: 42: 53.42$ & $+02: 37: 01.2$ & 4.34 & 168.2 & 20.02 & 1.62 & $1099 \pm 15$ & $1124 \pm 23$ & RedFinal & f06-48 & 0 \\
\hline $1.3: 23$ & $12: 42: 46.89$ & $+02: 37: 15.2$ & 4.08 & -169.5 & 20.50 & 1.73 & $901 \pm 25$ & $944 \pm 30$ & RedFinal & f06-50 & 0 \\
\hline $1.3: 25$ & $12: 42: 47.62$ & $+02: 37: 22.6$ & 3.93 & -171.8 & 20.15 & 1.50 & $1060 \pm 34$ & $1086 \pm 37$ & BlueFinal & $1.2: 23$ & 0 \\
\hline $1.3: 27$ & $12: 42: 51.66$ & $+02: 37: 30.7$ & 3.78 & 173.2 & 20.83 & 1.81 & $641 \pm 21$ & $694 \pm 37$ & RedFinal & $1.2: 25$ & 0 \\
\hline $1.3: 31$ & $12: 42: 55.12$ & $+02: 37: 43.8$ & 3.77 & 159.7 & 20.61 & 1.65 & $875 \pm 30$ & $909 \pm 37$ & RedFinal & f06-56 & 0 \\
\hline $1.3: 41$ & $12: 42: 49.82$ & $+02: 38: 18.6$ & 2.96 & -179.8 & 21.07 & 1.35 & $877 \pm 60$ & $877 \pm 60$ & BlueFinal & & 1 \\
\hline $1.3: 45$ & $12: 42: 53.10$ & $+02: 38: 33.8$ & 2.82 & 163.4 & 21.97 & 1.87 & $946 \pm 36$ & $946 \pm 36$ & RedFinal & & 1 \\
\hline $1.3: 48$ & $12: 42: 52.37$ & $+02: 39: 00.7$ & 2.34 & 164.5 & 20.58 & 1.44 & $992 \pm 25$ & $992 \pm 25$ & R2.5 & & 1 \\
\hline $1.3: 52$ & $12: 42: 46.04$ & $+02: 39: 15.9$ & 2.22 & -154.5 & 21.32 & 1.84 & $750 \pm 22$ & $747 \pm 29$ & R2.5 & f07-17 & 0 \\
\hline $1.3: 57$ & $12: 42: 52.31$ & $+02: 39: 39.2$ & 1.72 & 159.3 & 20.28 & 1.39 & $565 \pm 16$ & $565 \pm 16$ & R2.5 & & 1 \\
\hline $1.3: 58$ & $12: 42: 55.29$ & $+02: 39: 48.3$ & 1.99 & 137.2 & 19.21 & 1.21 & $1345 \pm 15$ & $1345 \pm 15$ & $\mathrm{R} 2.5$ & & 1 \\
\hline $1.3: 62$ & $12: 42: 55.45$ & $+02: 40: 05.4$ & 1.82 & 130.2 & 21.69 & 1.43 & $1230 \pm 30$ & $1230 \pm 30$ & $\mathrm{R} 2.5$ & & 1 \\
\hline $1.3: 64$ & $12: 42: 56.07$ & $+02: 40: 11.8$ & 1.88 & 124.6 & 20.94 & 1.14 & $592 \pm 36$ & $592 \pm 36$ & R2.5 & & 1 \\
\hline $1.3: 66$ & $12: 42: 56.17$ & $+02: 40: 17.8$ & 1.85 & 121.7 & 20.19 & 1.99 & $1134 \pm 21$ & $1134 \pm 21$ & R2.5 & & 1 \\
\hline $1.3: 69$ & $12: 42: 55.93$ & $+02: 40: 30.2$ & 1.69 & 116.8 & 21.79 & 0.85 & $514 \pm 42$ & $514 \pm 42$ & R2.5 & & 1 \\
\hline $1.3: 72$ & $12: 42: 59.20$ & $+02: 40: 45.4$ & 2.39 & 102.3 & 20.84 & 1.79 & $808 \pm 17$ & $808 \pm 17$ & R2.5 & & 1 \\
\hline $1.3: 73$ & $12: 42: 56.26$ & $+02: 40: 50.5$ & 1.65 & 104.9 & 20.81 & 1.31 & $1021 \pm 24$ & $1021 \pm 24$ & R2.5 & & 1 \\
\hline $1.3: 88$ & $12: 42: 55.25$ & $+02: 41: 38.5$ & 1.39 & 74.4 & 21.08 & 0.87 & $882 \pm 36$ & $882 \pm 36$ & R2.5 & & 1 \\
\hline $1.3: 95$ & $12: 42: 55.01$ & $+02: 42: 04.5$ & 1.52 & 57.8 & 20.61 & 1.76 & $1253 \pm 22$ & $1253 \pm 22$ & R2.5 & & 1 \\
\hline $1.3: 96$ & $12: 42: 54.80$ & $+02: 42: 08.4$ & 1.51 & 54.6 & 20.93 & 1.61 & $934 \pm 26$ & $934 \pm 26$ & R2.5 & & 1 \\
\hline $1.3: 99$ & $12: 42: 50.17$ & $+02: 42: 18.0$ & 1.04 & 4.1 & 20.63 & 1.47 & $618 \pm 20$ & $618 \pm 20$ & R2.5 & & 1 \\
\hline $1.3: 104$ & $12: 42: 53.27$ & $+02: 42: 40.5$ & 1.64 & 31.1 & 20.45 & 0.97 & $1107 \pm 33$ & $1107 \pm 33$ & R2.5 & & 1 \\
\hline $2.1: 77$ & $12: 42: 17.62$ & $+02: 39: 23.6$ & 8.27 & -103.1 & 21.65 & 1.71 & $946 \pm 38$ & $946 \pm 38$ & RedFinal & & 1 \\
\hline $2.1: 83$ & $12: 42: 23.15$ & $+02: 39: 44.5$ & 6.84 & -102.9 & 21.65 & 0.98 & $957 \pm 36$ & $1007 \pm 100$ & Blue & f08-41, f10-08 & 0 \\
\hline $2.1: 100$ & $12: 42: 17.51$ & $+02: 40: 52.6$ & 8.09 & -92.8 & 21.83 & 1.86 & $1258 \pm 46$ & $1258 \pm 46$ & RedFinal & & 1 \\
\hline $2.2: 38$ & $12: 42: 17.45$ & $+02: 36: 35.6$ & 9.35 & -120.0 & 19.92 & 1.11 & $850 \pm 44$ & $850 \pm 44$ & BlueFinal & & 1 \\
\hline $2.2: 59$ & $12: 42: 24.39$ & $+02: 38: 18.8$ & 7.01 & -114.9 & 20.85 & 1.23 & $1040 \pm 51$ & $1035 \pm 57$ & BlueFinal & f08-28 & 0 \\
\hline $3.1: 3$ & $12: 42: 33.92$ & $+02: 42: 55.7$ & 4.32 & -67.4 & 21.21 & 1.85 & $1165 \pm 22$ & $1165 \pm 22$ & RedFinal & & 1 \\
\hline $3.1: 4$ & $12: 42: 38.33$ & $+02: 42: 59.9$ & 3.36 & -59.0 & 21.43 & 1.62 & $947 \pm 45$ & $947 \pm 45$ & RedFinal & & 1 \\
\hline $3.1: 8$ & $12: 42: 32.14$ & $+02: 43: 16.9$ & 4.86 & -65.5 & 20.98 & 1.36 & $1186 \pm 26$ & $1186 \pm 26$ & BlueFinal & & 1 \\
\hline $3.1: 9$ & $12: 42: 34.76$ & $+02: 43: 20.8$ & 4.31 & -61.1 & 21.22 & 1.60 & $950 \pm 27$ & $950 \pm 27$ & RedFinal & & 1 \\
\hline $3.1: 10$ & $12: 42: 28.25$ & $+02: 43: 25.1$ & 5.81 & -68.3 & 19.88 & 1.60 & $550 \pm 30$ & $550 \pm 30$ & RedFinal & & 1 \\
\hline $3.1: 12$ & $12: 42: 30.52$ & $+02: 43: 30.0$ & 5.32 & -65.2 & 21.59 & 1.14 & $802 \pm 44$ & $802 \pm 44$ & BlueFinal & & 1 \\
\hline $3.1: 17$ & $12: 42: 28.85$ & $+02: 43: 49.8$ & 5.84 & -64.0 & 21.36 & 1.29 & $907 \pm 39$ & $980 \pm 45$ & BlueFinal & f10-45 & 0 \\
\hline $3.1: 19$ & $12: 42: 38.60$ & $+02: 43: 58.4$ & 3.90 & -46.1 & 19.81 & 1.77 & $692 \pm 19$ & $692 \pm 19$ & RedFinal & & 1 \\
\hline $3.1: 22$ & $12: 42: 33.11$ & $+02: 44: 08.0$ & 5.07 & -55.6 & 21.59 & 1.60 & $817 \pm 35$ & $817 \pm 35$ & RedFinal & & 1 \\
\hline $3.1: 24$ & $12: 42: 31.72$ & $+02: 44: 14.3$ & 5.42 & -56.7 & 21.40 & 1.56 & $522 \pm 29$ & $666 \pm 54$ & RedFinal & f09-04, f10-49 & 0 \\
\hline $3.1: 31$ & $12: 42: 30.85$ & $+02: 44: 41.4$ & 5.85 & -54.2 & 20.69 & 1.27 & $690 \pm 22$ & $685 \pm 34$ & BlueFinal & f09-07 & 0 \\
\hline $3.1: 38$ & $12: 42: 34.96$ & $+02: 45: 05.9$ & 5.34 & -44.2 & 21.88 & 1.18 & $541 \pm 36$ & $541 \pm 36$ & BlueFinal & & 1 \\
\hline $3.1: 45$ & $12: 42: 36.89$ & $+02: 45: 26.5$ & 5.29 & -37.8 & 19.86 & 1.34 & $915 \pm 26$ & $915 \pm 26$ & BlueFinal & & 1 \\
\hline $3.1: 47$ & $12: 42: 34.59$ & $+02: 45: 33.7$ & 5.75 & -41.6 & 20.70 & 1.63 & $857 \pm 29$ & $857 \pm 29$ & RedFinal & & 1 \\
\hline $3.1: 50$ & $12: 42: 38.70$ & $+02: 45: 58.3$ & 5.47 & -30.7 & 21.70 & 1.58 & $649 \pm 40$ & $661 \pm 46$ & RedFinal & f13-06 & 0 \\
\hline $3.1: 52$ & $12: 42: 37.27$ & $+02: 46: 04.1$ & 5.74 & -33.2 & 21.53 & 1.65 & $1046 \pm 42$ & $1046 \pm 42$ & RedFinal & & 1 \\
\hline $3.1: 57$ & $12: 42: 31.61$ & $+02: 46: 22.1$ & 6.84 & -41.8 & 21.58 & 1.28 & $972 \pm 41$ & $972 \pm 41$ & BlueFinal & & 1 \\
\hline $3.1: 62$ & $12: 42: 36.38$ & $+02: 46: 37.4$ & 6.33 & -32.2 & 19.93 & 1.72 & $1027 \pm 20$ & $1027 \pm 20$ & RedFinal & & 1 \\
\hline $3.1: 65$ & $12: 42: 34.60$ & $+02: 46: 47.7$ & 6.72 & -34.6 & 20.51 & 1.57 & $902 \pm 21$ & $902 \pm 21$ & RedFinal & & 1 \\
\hline $3.1: 69$ & $12: 42: 34.82$ & $+02: 46: 59.5$ & 6.85 & -33.3 & 20.46 & 1.20 & $1423 \pm 25$ & $1428 \pm 38$ & Blue & f13-15 & 0 \\
\hline $3.1: 80$ & $12: 42: 32.39$ & $+02: 47: 43.2$ & 7.79 & -34.1 & 20.73 & 1.57 & $778 \pm 19$ & $778 \pm 19$ & RedFinal & & 1 \\
\hline $3.1: 81$ & $12: 42: 38.13$ & $+02: 47: 47.5$ & 7.15 & -24.2 & 21.12 & 1.23 & $1014 \pm 25$ & $1014 \pm 25$ & BlueFinal & & 1 \\
\hline $3.1: 92$ & $12: 42: 39.48$ & $+02: 48: 33.9$ & 7.75 & -19.6 & 20.72 & 1.22 & $729 \pm 20$ & $729 \pm 20$ & BlueFinal & & 1 \\
\hline $3.1: 112$ & $12: 42: 37.98$ & $+02: 49: 46.8$ & 9.02 & -19.2 & 21.86 & 1.17 & $691 \pm 55$ & $691 \pm 55$ & BlueFinal & & 1 \\
\hline $3.2: 1$ & $12: 42: 31.95$ & $+02: 42: 49.0$ & 4.74 & -70.9 & 21.59 & 0.96 & $1281 \pm 40$ & $1281 \pm 40$ & BlueFinal & & 1 \\
\hline
\end{tabular}


Table C.1. continued.

\begin{tabular}{|c|c|c|c|c|c|c|c|c|c|c|c|}
\hline ID & $\begin{array}{c}\text { RA } \\
(\mathrm{J} 2000)\end{array}$ & $\begin{array}{c}\text { Dec } \\
(\mathrm{J} 2000)\end{array}$ & $\begin{array}{c}R_{4636} \\
{[\operatorname{arcmin}]}\end{array}$ & $\begin{array}{c}\mathrm{PA} \\
{[\mathrm{deg}]}\end{array}$ & $\begin{array}{c}m_{R} \\
{[\mathrm{mag}]}\end{array}$ & $\begin{array}{l}C-R \\
{[\mathrm{mag}]}\end{array}$ & $\begin{array}{c}v_{\mathrm{spec}} \\
{\left[\mathrm{km} \mathrm{s}^{-1}\right]}\end{array}$ & $\begin{array}{c}v_{\text {final }} \\
{\left[\mathrm{km} \mathrm{s}^{-1}\right]}\end{array}$ & Sample & Cross-Identification(s) & $1 \mathrm{st}$ \\
\hline $3.2: 9$ & $12: 42: 33.08$ & $+02: 43: 16.8$ & 4.65 & -64.3 & 21.69 & 1.32 & $1175 \pm 28$ & $1175 \pm 28$ & BlueFinal & & 1 \\
\hline $3.2: 12$ & $12: 42: 28.58$ & $+02: 43: 27.2$ & 5.75 & -67.6 & 21.32 & 1.06 & $912 \pm 43$ & $912 \pm 43$ & BlueFinal & & 1 \\
\hline $3.2: 20$ & $12: 42: 26.23$ & $+02: 43: 57.8$ & 6.49 & -65.4 & 21.29 & 1.23 & $860 \pm 46$ & $861 \pm 78$ & Blue & f09-02, f10-47 & 0 \\
\hline $3.2: 31$ & $12: 42: 35.06$ & $+02: 44: 31.4$ & 4.93 & -48.6 & 21.83 & 1.72 & $1092 \pm 43$ & $1144 \pm 53$ & RedFinal & f07-72 & 0 \\
\hline $3.2: 34$ & $12: 42: 29.26$ & $+02: 44: 42.1$ & 6.19 & -56.3 & 21.17 & 1.64 & $1002 \pm 29$ & $1002 \pm 29$ & RedFinal & & 1 \\
\hline $3.2: 37$ & $12: 42: 27.33$ & $+02: 44: 52.6$ & 6.69 & -57.3 & 20.69 & 1.31 & $736 \pm 22$ & $798 \pm 65$ & Blue & $\mathrm{f} 10-57$ & 0 \\
\hline $3.2: 50$ & $12: 42: 35.10$ & $+02: 45: 35.4$ & 5.68 & -40.5 & 22.05 & 2.00 & $1050 \pm 26$ & $1050 \pm 26$ & RedFinal & & 1 \\
\hline $3.2: 61$ & $12: 42: 34.47$ & $+02: 46: 35.5$ & 6.57 & -35.8 & 20.70 & 1.71 & $1273 \pm 21$ & $1273 \pm 21$ & RedFinal & & 1 \\
\hline $3.2: 65$ & $12: 42: 36.97$ & $+02: 46: 53.5$ & 6.48 & -29.8 & 20.95 & 1.04 & $1441 \pm 28$ & $1441 \pm 28$ & Blue & & 1 \\
\hline $3.2: 106$ & $12: 42: 30.95$ & $+02: 49: 29.5$ & 9.49 & -29.9 & 20.82 & 1.63 & $916 \pm 25$ & $916 \pm 25$ & RedFinal & & 1 \\
\hline $4.1: 16$ & $12: 42: 32.21$ & $+02: 50: 50.1$ & 10.54 & -24.7 & 22.03 & 1.81 & $869 \pm 50$ & $869 \pm 50$ & RedFinal & & 1 \\
\hline $4.1: 33$ & $12: 42: 29.38$ & $+02: 52: 00.0$ & 11.89 & -25.5 & 21.93 & 1.84 & $760 \pm 64$ & $774 \pm 75$ & RedFinal & f13-61 & 0 \\
\hline $5.1: 1$ & $12: 42: 54.62$ & $+02: 43: 20.3$ & 2.39 & 29.8 & 21.99 & 0.74 & $1169 \pm 35$ & $1169 \pm 35$ & $\mathrm{R} 2.5$ & & 1 \\
\hline $5.1: 18$ & $12: 42: 57.63$ & $+02: 44: 23.8$ & 3.68 & 31.8 & 20.96 & 1.14 & $963 \pm 23$ & $963 \pm 23$ & BlueFinal & & 1 \\
\hline $5.1: 22$ & $12: 42: 55.55$ & $+02: 44: 39.2$ & 3.67 & 22.7 & 20.45 & 1.26 & $1043 \pm 48$ & $1045 \pm 78$ & Blue & f03-12 & 0 \\
\hline $5.1: 33$ & $12: 42: 59.56$ & $+02: 45: 13.0$ & 4.63 & 31.5 & 21.87 & 1.87 & $998 \pm 31$ & $998 \pm 31$ & RedFinal & & 1 \\
\hline $5.1: 38$ & $12: 43: 05.34$ & $+02: 45: 31.3$ & 5.75 & 42.2 & 21.86 & 1.79 & $854 \pm 29$ & $825 \pm 64$ & RedFinal & f03-20 & 0 \\
\hline $5.1: 43$ & $12: 43: 05.46$ & $+02: 45: 48.7$ & 5.98 & 40.6 & 19.91 & 1.30 & $928 \pm 23$ & $928 \pm 23$ & BlueFinal & & 1 \\
\hline $5.1: 46$ & $12: 42: 57.64$ & $+02: 46: 01.8$ & 5.14 & 22.2 & 21.50 & 1.37 & $1102 \pm 31$ & $1102 \pm 31$ & BlueFinal & & 1 \\
\hline $5.1: 48$ & $12: 42: 58.75$ & $+02: 46: 30.9$ & 5.70 & 22.9 & 19.41 & 1.22 & $1085 \pm 13$ & $1085 \pm 13$ & BlueFinal & & 1 \\
\hline $5.1: 49$ & $12: 43: 02.69$ & $+02: 46: 37.3$ & 6.24 & 30.9 & 20.73 & 1.15 & $783 \pm 23$ & $783 \pm 23$ & BlueFinal & & 1 \\
\hline $5.1: 52$ & $12: 43: 08.34$ & $+02: 46: 49.9$ & 7.23 & 39.6 & 19.33 & 1.40 & $695 \pm 16$ & $721 \pm 45$ & BlueFinal & f03-32 & 0 \\
\hline $5.1: 58$ & $12: 42: 53.70$ & $+02: 47: 11.7$ & 6.00 & 9.2 & 20.72 & 1.23 & $771 \pm 22$ & $771 \pm 22$ & BlueFinal & & 1 \\
\hline $5.1: 60$ & $12: 43: 04.55$ & $+02: 47: 17.4$ & 7.05 & 31.3 & 20.59 & 1.14 & $661 \pm 35$ & $661 \pm 35$ & BlueFinal & & 1 \\
\hline $5.1: 63$ & $12: 43: 03.49$ & $+02: 47: 27.1$ & 7.06 & 28.8 & 20.99 & 1.99 & $919 \pm 17$ & $919 \pm 17$ & RedFinal & & 1 \\
\hline $5.1: 69$ & $12: 42: 56.19$ & $+02: 47: 50.1$ & 6.76 & 13.5 & 19.54 & 1.54 & $1048 \pm 15$ & $1048 \pm 15$ & BlueFinal & & 1 \\
\hline $5.1: 83$ & $12: 43: 03.00$ & $+02: 48: 54.0$ & 8.31 & 23.2 & 21.63 & 1.66 & $658 \pm 38$ & $658 \pm 38$ & RedFinal & & 1 \\
\hline $5.1: 91$ & $12: 43: 01.02$ & $+02: 49: 19.9$ & 8.53 & 19.0 & 21.39 & 1.28 & $1014 \pm 46$ & $1054 \pm 102$ & Blue & f03-54 & 0 \\
\hline $6.1: 45$ & $12: 43: 08.76$ & $+02: 39: 11.0$ & 5.16 & 113.8 & 19.85 & 1.22 & $1048 \pm 21$ & $1043 \pm 30$ & BlueFinal & f12-19 & 0 \\
\hline $6.1: 67$ & $12: 43: 20.79$ & $+02: 40: 31.5$ & 7.76 & 95.5 & 21.21 & 1.20 & $724 \pm 43$ & $724 \pm 43$ & BlueFinal & & 1 \\
\hline $6.1: 88$ & $12: 43: 08.88$ & $+02: 41: 55.0$ & 4.79 & 82.2 & 19.99 & 1.40 & $618 \pm 25$ & $618 \pm 25$ & BlueFinal & & 1 \\
\hline $6.1: 92$ & $12: 43: 09.04$ & $+02: 42: 06.1$ & 4.86 & 80.1 & 20.02 & 1.54 & $698 \pm 23$ & $713 \pm 30$ & BlueFinal & $\mathrm{f} 02-48, \mathrm{f} 12-44$ & 0 \\
\hline $6.2: 16$ & $12: 43: 05.96$ & $+02: 36: 50.6$ & 5.98 & 137.7 & 21.69 & 1.18 & $868 \pm 29$ & $868 \pm 29$ & BlueFinal & & 1 \\
\hline $6.2: 18$ & $12: 43: 09.78$ & $+02: 36: 57.8$ & 6.58 & 130.9 & 21.77 & 1.24 & $670 \pm 37$ & $670 \pm 37$ & BlueFinal & & 1 \\
\hline $6.2: 23$ & $12: 43: 08.11$ & $+02: 37: 14.4$ & 6.08 & 131.5 & $\ldots$ & $\ldots$ & $.1316 \pm 30$ & $1316 \pm 30$ & NoPhot & & 1 \\
\hline $6.2: 30$ & $12: 43: 12.02$ & $+02: 37: 38.4$ & 6.61 & 123.2 & 20.29 & 1.49 & $924 \pm 15$ & $924 \pm 15$ & BlueFinal & & 1 \\
\hline $6.2: 35$ & $12: 43: 07.95$ & $+02: 37: 55.3$ & 5.62 & 126.5 & 21.11 & 1.11 & $818 \pm 33$ & $818 \pm 33$ & BlueFinal & & 1 \\
\hline $6.2: 57$ & $12: 43: 17.10$ & $+02: 39: 24.9$ & 7.05 & 105.2 & 21.32 & 1.26 & $1309 \pm 62$ & $1348 \pm 76$ & Blue & $\mathrm{f} 12-23$ & 0 \\
\hline $6.2: 59$ & $12: 43: 09.28$ & $+02: 39: 33.2$ & 5.14 & 109.5 & 19.85 & 1.62 & $990 \pm 15$ & $979 \pm 15$ & RedFinal & f12-24 & 0 \\
\hline $6.2: 63$ & $12: 43: 06.55$ & $+02: 39: 53.4$ & 4.39 & 108.3 & 19.76 & 1.47 & $790 \pm 17$ & $790 \pm 17$ & BlueFinal & & 1 \\
\hline $6.2: 65$ & $12: 43: 11.42$ & $+02: 40: 01.3$ & 5.52 & 103.0 & 20.59 & 1.18 & $940 \pm 26$ & $940 \pm 26$ & BlueFinal & & 1 \\
\hline $6.2: 81$ & $12: 43: 06.56$ & $+02: 40: 59.1$ & 4.18 & 93.9 & 22.24 & 2.15 & $959 \pm 37$ & $959 \pm 37$ & RedFinal & & 1 \\
\hline $6.2: 86$ & $12: 43: 14.28$ & $+02: 41: 20.4$ & 6.10 & 89.3 & 20.68 & 1.41 & $986 \pm 25$ & $986 \pm 25$ & BlueFinal & & 1 \\
\hline $6.2: 87$ & $12: 43: 08.26$ & $+02: 41: 25.4$ & 4.60 & 88.0 & 20.64 & 1.10 & $791 \pm 19$ & $791 \pm 19$ & BlueFinal & & 1 \\
\hline $6.2: 95$ & $12: 43: 14.68$ & $+02: 41: 53.6$ & 6.23 & 84.2 & 21.23 & 2.08 & $614 \pm 28$ & $622 \pm 35$ & RedFinal & f12-42 & 0 \\
\hline $6.2: 104$ & $12: 43: 17.30$ & $+02: 42: 29.5$ & 6.96 & 79.9 & 21.04 & 1.73 & $746 \pm 21$ & $746 \pm 21$ & RedFinal & & 1 \\
\hline $6.2: 108$ & $12: 43: 06.79$ & $+02: 42: 43.9$ & 4.47 & 70.9 & 20.13 & 1.15 & $885 \pm 26$ & $881 \pm 35$ & BlueFinal & f02-55 & 0 \\
\hline $7.2: 2$ & $12: 43: 21.19$ & $+02: 28: 52.0$ & 14.66 & 147.8 & 21.80 & 1.28 & $535 \pm 88$ & $535 \pm 88$ & Blue & & 1 \\
\hline $7.2: 23$ & $12: 43: 32.17$ & $+02: 30: 01.0$ & 15.43 & 136.8 & 22.73 & 1.15 & $1051 \pm 68$ & $1051 \pm 68$ & Blue & & 1 \\
\hline
\end{tabular}


Y. Schuberth et al.: Dynamics of the NGC 4636 globular cluster system. II.

Appendix D: Foreground star velocities

Table D.1. Catalogue of foreground star velocities.

\begin{tabular}{|c|c|c|c|c|c|c|c|c|c|c|}
\hline ID & $\begin{array}{l}\text { RA } \\
(\mathrm{J} 2000) \\
(2)\end{array}$ & $\begin{array}{l}\text { Dec } \\
(\mathrm{J} 2000) \\
(3)\end{array}$ & $\begin{array}{c}R_{4636} \\
\text { [arcmin] } \\
\quad(4)\end{array}$ & $\begin{array}{l}\text { PA } \\
{[\mathrm{deg}]} \\
(5)\end{array}$ & $\begin{array}{c}m_{R} \\
{[\mathrm{mag}]} \\
(6)\end{array}$ & $\begin{array}{c}C-R \\
{[\mathrm{mag}]} \\
(7)\end{array}$ & $\begin{array}{c}v_{\text {spec }} \\
{\left[\mathrm{km} \mathrm{s}^{-1}\right]} \\
(8)\end{array}$ & $\begin{array}{c}v_{\text {final }} \\
{\left[\mathrm{km} \mathrm{s}^{-1}\right]} \\
(9)\end{array}$ & $\begin{array}{c}\text { Cross-Identification(s) } \\
\qquad(10)\end{array}$ & (11) \\
\hline f01-01 & $12: 43: 07.15$ & $+02: 32: 54.3$ & 9.41 & 152.7 & 19.91 & 0.79 & $249 \pm 35$ & $249 \pm 35$ & & 1 \\
\hline f01-13 & $12: 43: 09.22$ & $+02: 34: 27.0$ & 8.36 & 144.7 & 22.04 & 3.46 & $-68 \pm 53$ & $-68 \pm 53$ & & 1 \\
\hline f01-18 & $12: 43: 07.75$ & $+02: 35: 02.3$ & 7.66 & 144.4 & 21.91 & 2.46 & $-167 \pm 58$ & $-167 \pm 58$ & & 1 \\
\hline f01-19 & $12: 43: 06.68$ & $+02: 35: 08.9$ & 7.42 & 145.5 & 21.86 & 2.70 & $86 \pm 41$ & $86 \pm 41$ & & 1 \\
\hline f01-23 & $12: 43: 07.00$ & $+02: 35: 39.2$ & 7.06 & 142.7 & 21.86 & 1.72 & $-23 \pm 29$ & $-23 \pm 29$ & & 1 \\
\hline f01-27 & $12: 43: 10.05$ & $+02: 36: 25.4$ & 6.99 & 133.9 & 18.55 & 1.24 & $-44 \pm 13$ & $-51 \pm 21$ & $6.2: 9$ & 1 \\
\hline f01-29 & $12: 42: 59.66$ & $+02: 36: 40.3$ & 5.21 & 152.0 & & 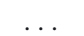 & $-83 \pm 46$ & $-46 \pm 55$ & f06-44 & 1 \\
\hline f01-39 & $12: 43: 10.33$ & $+02: 37: 39.9$ & 6.25 & 125.2 & 22.05 & 0.67 & $68 \pm 58$ & $22 \pm 98$ & f12-06 & 1 \\
\hline f01-47 & $12: 43: 12.07$ & $+02: 38: 39.8$ & 6.13 & 115.2 & $\ldots$ & $\ldots$ & $6 \pm 17$ & $6 \pm 17$ & & 1 \\
\hline f01-52 & $12: 43: 06.34$ & $+02: 39: 10.2$ & 4.62 & 117.0 & & & $19 \pm 33$ & $19 \pm 33$ & & 1 \\
\hline f02-06 & $12: 42: 55.68$ & $+02: 37: 48.4$ & 3.75 & 157.2 & 22.01 & 0.89 & $67 \pm 79$ & $146 \pm 110$ & f06-57 & 1 \\
\hline f02-36 & 12:43:08.09 & $+02: 40: 51.6$ & 4.57 & 95.1 & 19.82 & 0.79 & $-198 \pm 24$ & $-196 \pm 32$ & $6.2: 79$ & 1 \\
\hline f02-46 & $12: 42: 58.61$ & $+02: 41: 52.3$ & 2.26 & 74.5 & 18.93 & 0.55 & $48 \pm 62$ & $71 \pm 108$ & $1.3: 91$ & 1 \\
\hline f02-67 & 12:43:05.04 & $+02: 44: 03.4$ & 4.70 & 53.6 & 18.88 & 2.27 & $-108 \pm 31$ & $-89 \pm 54$ & $5.1: 13$ & 1 \\
\hline f03-28 & $12: 43: 10.67$ & $+02: 46: 14.8$ & 7.20 & 46.2 & 19.05 & 3.05 & $-26 \pm 55$ & $-26 \pm 55$ & & 1 \\
\hline f03-30 & $12: 43: 00.35$ & $+02: 46: 31.3$ & 5.87 & 26.5 & 20.31 & 0.42 & $203 \pm 84$ & $203 \pm 84$ & & 1 \\
\hline f03-31 & 12:43:01.06 & $+02: 46: 45.6$ & 6.16 & 27.0 & 21.01 & 3.38 & $94 \pm 62$ & $94 \pm 62$ & & 1 \\
\hline f03-42 & $12: 43: 08.32$ & $+02: 47: 48.3$ & 8.00 & 35.2 & 20.95 & 0.69 & $159 \pm 75$ & $159 \pm 75$ & & 1 \\
\hline f03-44 & $12: 42: 59.94$ & $+02: 48: 04.8$ & 7.26 & 20.3 & 21.93 & 1.47 & $192 \pm 46$ & $192 \pm 46$ & & 1 \\
\hline f03-45 & $12: 43: 00.47$ & $+02: 48: 15.3$ & 7.47 & 20.7 & 20.06 & 2.72 & $21 \pm 79$ & $17 \pm 93$ & $5.1: 75$ & 1 \\
\hline f03-47 & $12: 43: 09.11$ & $+02: 48: 28.0$ & 8.66 & 33.7 & 18.24 & -0.19 & $88 \pm 61$ & $108 \pm 65$ & $5.1: 77$ & 1 \\
\hline f04-01 & $12: 42: 54.48$ & $+02: 45: 56.4$ & 4.81 & 13.8 & 21.92 & 1.94 & $151 \pm 55$ & $151 \pm 55$ & & 1 \\
\hline f04-13 & $12: 42: 46.86$ & $+02: 47: 01.2$ & 5.80 & -7.4 & 19.37 & 2.06 & $-15 \pm 104$ & $24 \pm 110$ & f05-63 & 1 \\
\hline f04-15 & $12: 42: 43.50$ & $+02: 47: 19.9$ & 6.27 & -14.7 & 21.70 & 1.16 & $255 \pm 45$ & $255 \pm 45$ & & 1 \\
\hline f04-18 & $12: 42: 45.61$ & $+02: 47: 34.0$ & 6.39 & -9.6 & 21.94 & 0.94 & $34 \pm 65$ & $34 \pm 65$ & & 1 \\
\hline f04-25 & $12: 42: 48.05$ & $+02: 48: 20.6$ & 7.09 & -3.7 & 22.18 & 1.39 & $163 \pm 71$ & $163 \pm 71$ & & 1 \\
\hline f04-27 & $12: 42: 50.32$ & $+02: 48: 32.6$ & 7.28 & 0.9 & 22.05 & 1.25 & $-3 \pm 70$ & $-3 \pm 70$ & & 1 \\
\hline f04-30 & $12: 42: 46.62$ & $+02: 49: 02.0$ & 7.81 & -6.0 & 21.44 & 0.67 & $44 \pm 33$ & $44 \pm 33$ & & 1 \\
\hline f04-32 & $12: 42: 41.32$ & $+02: 49: 20.6$ & 8.35 & -14.8 & 22.21 & 0.82 & $-31 \pm 58$ & $-31 \pm 58$ & & 1 \\
\hline f04-33 & $12: 42: 43.53$ & $+02: 49: 24.4$ & 8.29 & -11.0 & 19.11 & 3.40 & $-10 \pm 57$ & $-10 \pm 57$ & & 1 \\
\hline f04-43 & $12: 42: 40.69$ & $+02: 50: 23.9$ & 9.42 & -14.1 & 21.35 & 1.05 & $-3 \pm 39$ & $22 \pm 44$ & f13-48 & 1 \\
\hline f04-44 & $12: 42: 38.49$ & $+02: 50: 31.6$ & 9.69 & -17.1 & 20.48 & 1.36 & $57 \pm 19$ & $74 \pm 28$ & f13-49 & 1 \\
\hline f04-46 & $12: 42: 54.41$ & $+02: 50: 46.7$ & 9.58 & 6.8 & 19.17 & 0.42 & $25 \pm 22$ & $25 \pm 22$ & & 1 \\
\hline f04-54 & $12: 42: 51.44$ & $+02: 51: 35.7$ & 10.34 & 2.2 & 20.14 & 1.43 & $244 \pm 19$ & $244 \pm 19$ & & 1 \\
\hline f04-57 & $12: 42: 43.44$ & $+02: 51: 49.7$ & 10.68 & -8.6 & 19.07 & 1.10 & $37 \pm 14$ & $27 \pm 22$ & f13-59 & 1 \\
\hline f04-58 & $12: 42: 54.83$ & $+02: 51: 57.0$ & 10.75 & 6.6 & 19.28 & 3.11 & $-4 \pm 84$ & $-4 \pm 84$ & & 1 \\
\hline f04-62 & $12: 42: 53.84$ & $+02: 52: 26.2$ & 11.21 & 5.1 & 19.10 & 2.99 & $-40 \pm 57$ & $-40 \pm 57$ & & 1 \\
\hline f05-24 & $12: 42: 47.99$ & $+02: 42: 33.0$ & 1.37 & -20.1 & 20.71 & 2.11 & $49 \pm 55$ & $49 \pm 55$ & & 1 \\
\hline f05-34 & $12: 42: 41.20$ & $+02: 43: 51.9$ & 3.38 & -39.8 & 19.90 & 2.55 & $134 \pm 31$ & $134 \pm 31$ & & 1 \\
\hline f05-38 & $12: 42: 51.06$ & $+02: 44: 12.5$ & 2.96 & 5.8 & 22.04 & 0.86 & $60 \pm 37$ & $60 \pm 37$ & & 1 \\
\hline f05-40 & $12: 42: 50.44$ & $+02: 44: 28.0$ & 3.20 & 2.5 & 19.32 & 1.24 & $127 \pm 13$ & $127 \pm 13$ & & 1 \\
\hline f05-43 & $12: 42: 39.61$ & $+02: 44: 48.4$ & 4.37 & -35.9 & 19.27 & 0.22 & $-28 \pm 16$ & $-28 \pm 16$ & & 1 \\
\hline f05-44 & $12: 42: 46.72$ & $+02: 44: 53.9$ & 3.72 & -12.2 & 18.38 & 1.39 & $68 \pm 53$ & $68 \pm 53$ & & 1 \\
\hline f05-50 & $12: 42: 45.91$ & $+02: 45: 37.2$ & 4.46 & -12.8 & 20.42 & 3.24 & $121 \pm 57$ & $121 \pm 57$ & & 1 \\
\hline f05-59 & $12: 42: 42.68$ & $+02: 46: 33.0$ & 5.58 & -18.8 & 18.36 & 1.92 & $-29 \pm 31$ & $-29 \pm 31$ & & 1 \\
\hline f05-63 & $12: 42: 46.86$ & $+02: 47: 01.2$ & 5.80 & -7.4 & 19.37 & 2.06 & $62 \pm 34$ & $24 \pm 110$ & f04-13 & 0 \\
\hline f06-01 & $12: 43: 02.79$ & $+02: 31: 58.5$ & 9.84 & 160.8 & 19.61 & 2.24 & $-47 \pm 25$ & $-47 \pm 25$ & & 1 \\
\hline f06-02 & $12: 42: 54.07$ & $+02: 32: 05.8$ & 9.23 & 173.5 & 20.80 & 2.57 & $51 \pm 51$ & $51 \pm 51$ & & 1 \\
\hline f06-04 & $12: 42: 49.23$ & $+02: 32: 19.4$ & 8.94 & -179.0 & 22.68 & -0.41 & $33 \pm 66$ & $33 \pm 66$ & & 1 \\
\hline f06-12 & 12:43:00.96 & $+02: 33: 10.5$ & 8.55 & 161.1 & 20.44 & 2.83 & $40 \pm 42$ & $40 \pm 42$ & & 1 \\
\hline f06-18 & $12: 42: 51.90$ & $+02: 33: 47.0$ & 7.50 & 176.1 & 20.91 & 1.74 & $196 \pm 33$ & $196 \pm 33$ & & 1 \\
\hline
\end{tabular}

Notes. Column (1) gives the spectrum identifier (mask:slit). Note that the new data have the prefix "f". For the purpose of cross-referencing, the data from Paper I are also included in this table. Columns (2) and (3) are the right ascension and declination, respectively (for objects with Washington photometry, the coordinates are those from Dirsch et al. 2005; for objects not in the photometric catalogue, the values were estimated from the pre-images). The projected distance from the centre of NGC 4636 (in units of arcminutes) is given in Col. (4). Column (5) is the position angle (measured North over East). Columns (6) and (7) are the magnitude and colour as given by Dirsch et al. (2005). For the new objects, Col. (8) gives the heliocentric velocity measured for a given spectrum, for the data from Paper I, the final velocity given there is quoted. Column (9) gives the final velocity used in this study, for objects for which more than one spectrum was obtained, the error-weighted mean is given. Column (10) gives the cross-identifications within this list The entry in Col. (11) is "1" for the first appearance of an object in this table. All subsequent entries for the same object are labelled " 0 ". 
Table D.1. continued.

\begin{tabular}{|c|c|c|c|c|c|c|c|c|c|c|}
\hline ID & $\begin{array}{c}\text { RA } \\
(\mathrm{J} 2000)\end{array}$ & $\begin{array}{c}\text { Dec } \\
(\mathrm{J} 2000)\end{array}$ & $\begin{array}{c}R_{4636} \\
{[\operatorname{arcmin}]}\end{array}$ & $\begin{array}{c}\text { PA } \\
{[\mathrm{deg}]}\end{array}$ & $\begin{array}{c}m_{R} \\
{[\mathrm{mag}]}\end{array}$ & $\begin{array}{l}C-R \\
{[\mathrm{mag}]}\end{array}$ & $\begin{array}{c}v_{\mathrm{spec}} \\
{\left[\mathrm{km} \mathrm{s}^{-1}\right]}\end{array}$ & $\begin{array}{c}v_{\text {final }} \\
{\left[\mathrm{km} \mathrm{s}^{-1}\right]}\end{array}$ & Cross-Identification(s) & 1 st \\
\hline f06-26 & 12:43:01.49 & $+02: 34: 40.3$ & 7.21 & 156.2 & 18.84 & -0.42 & $89 \pm 28$ & $89 \pm 28$ & & 1 \\
\hline f06-27 & $12: 42: 47.92$ & $+02: 34: 47.4$ & 6.49 & -175.7 & 22.01 & 1.88 & $-57 \pm 86$ & $-57 \pm 86$ & & 1 \\
\hline f06-31 & $12: 42: 53.39$ & $+02: 35: 22.1$ & 5.96 & 171.5 & 21.84 & 0.95 & $39 \pm 53$ & $39 \pm 53$ & & 1 \\
\hline f06-35 & $12: 42: 46.16$ & $+02: 35: 45.8$ & 5.58 & -170.4 & 18.45 & 1.76 & $57 \pm 40$ & $57 \pm 40$ & & 1 \\
\hline f06-40 & $12: 42: 46.09$ & $+02: 36: 13.8$ & 5.12 & -169.4 & 20.47 & 2.18 & $66 \pm 24$ & $66 \pm 24$ & & 1 \\
\hline f06-44 & $12: 42: 59.66$ & $+02: 36: 40.3$ & 5.21 & 152.0 & .. & $\ldots$ & $-8 \pm 31$ & $-46 \pm 55$ & f01-29 & 0 \\
\hline f06-51 & $12: 42: 52.90$ & $+02: 37: 19.5$ & 4.01 & 169.1 & 21.33 & 1.29 & $253 \pm 30$ & $215 \pm 83$ & $1.1: 22$ & 1 \\
\hline f06-57 & $12: 42: 55.68$ & $+02: 37: 48.4$ & 3.75 & 157.2 & 22.01 & 0.89 & $225 \pm 76$ & $146 \pm 110$ & f02-06 & 0 \\
\hline f06-59 & $12: 42: 56.41$ & $+02: 38: 01.6$ & 3.63 & 153.2 & 18.44 & 1.22 & $44 \pm 27$ & $44 \pm 27$ & & 1 \\
\hline f06-69 & $12: 42: 48.55$ & $+02: 38: 48.1$ & 2.49 & -172.4 & 21.61 & 1.43 & $-26 \pm 59$ & $-26 \pm 59$ & & 1 \\
\hline f07-01 & $12: 42: 44.85$ & $+02: 37: 55.4$ & 3.57 & -159.4 & 20.68 & 3.56 & $-22 \pm 40$ & $-22 \pm 40$ & & 1 \\
\hline f07-11 & $12: 42: 49.40$ & $+02: 38: 43.2$ & 2.55 & -177.4 & 22.36 & 1.45 & $248 \pm 121$ & $248 \pm 121$ & & 1 \\
\hline f07-14 & $12: 42: 43.72$ & $+02: 38: 57.1$ & 2.78 & -146.4 & 19.91 & 2.97 & $69 \pm 32$ & $69 \pm 32$ & & 1 \\
\hline f07-16 & $12: 42: 37.77$ & $+02: 39: 09.6$ & 3.68 & -124.9 & 21.70 & 1.58 & $100 \pm 36$ & $80 \pm 54$ & f08-36 & 1 \\
\hline f07-24 & $12: 42: 37.75$ & $+02: 39: 56.3$ & 3.31 & -113.7 & 21.69 & 1.82 & $-108 \pm 47$ & $-148 \pm 54$ & f08-43 & 1 \\
\hline f07-41 & $12: 42: 35.25$ & $+02: 41: 45.8$ & 3.68 & -82.3 & 20.13 & 1.74 & $34 \pm 27$ & $-18 \pm 31$ & f08-64 & 1 \\
\hline f07-48 & $12: 42: 32.67$ & $+02: 42: 25.5$ & 4.45 & -74.9 & 18.68 & 0.75 & $129 \pm 26$ & $129 \pm 26$ & & 1 \\
\hline f07-67 & $12: 42: 34.62$ & $+02: 44: 02.3$ & 4.71 & -54.0 & 19.19 & 1.70 & $81 \pm 17$ & $41 \pm 46$ & $3.1: 20$ & 1 \\
\hline f07-71 & $12: 42: 31.75$ & $+02: 44: 24.9$ & 5.51 & -55.2 & 18.68 & -0.99 & $28 \pm 16$ & $28 \pm 16$ & & 1 \\
\hline f08-01 & $12: 42: 25.58$ & $+02: 35: 06.9$ & 8.64 & -135.4 & 21.94 & 0.84 & $-4 \pm 64$ & $-4 \pm 64$ & & 1 \\
\hline f08-04 & $12: 42: 27.22$ & $+02: 35: 25.3$ & 8.13 & -135.9 & 21.55 & 3.18 & $31 \pm 53$ & $31 \pm 53$ & & 1 \\
\hline f08-09 & $12: 42: 28.48$ & $+02: 35: 50.0$ & 7.62 & -135.5 & 21.05 & 2.32 & $-45 \pm 58$ & $-108 \pm 74$ & $2.2: 26$ & 1 \\
\hline f08-10 & $12: 42: 23.54$ & $+02: 35: 54.0$ & 8.49 & -129.2 & 21.82 & 1.90 & $-84 \pm 68$ & $-84 \pm 68$ & & 1 \\
\hline f08-11 & $12: 42: 28.31$ & $+02: 36: 00.8$ & 7.52 & -134.3 & 21.32 & 0.72 & $-26 \pm 58$ & $-26 \pm 58$ & & 1 \\
\hline f08-12 & $12: 42: 34.48$ & $+02: 36: 04.1$ & 6.46 & -143.5 & 21.77 & 0.63 & $-64 \pm 119$ & $-64 \pm 119$ & & 1 \\
\hline f08-13 & $12: 42: 25.40$ & $+02: 36: 19.2$ & 7.86 & -129.0 & 22.49 & 0.95 & $347 \pm 66$ & $347 \pm 66$ & & 1 \\
\hline f08-17 & $12: 42: 38.30$ & $+02: 36: 50.7$ & 5.28 & -146.8 & 21.89 & 0.68 & $111 \pm 62$ & $111 \pm 62$ & & 1 \\
\hline f08-22 & $12: 42: 32.93$ & $+02: 37: 28.3$ & 5.68 & -131.9 & 21.54 & 0.61 & $-209 \pm 86$ & $-209 \pm 86$ & & 1 \\
\hline f08-23 & $12: 42: 33.26$ & $+02: 37: 32.5$ & 5.58 & -131.9 & 19.80 & 1.15 & $-27 \pm 47$ & $-27 \pm 47$ & & 1 \\
\hline f08-30 & $12: 42: 32.53$ & $+02: 38: 32.6$ & 5.12 & -122.2 & 22.23 & 2.33 & $-94 \pm 64$ & $-94 \pm 64$ & & 1 \\
\hline f08-35 & $12: 42: 22.51$ & $+02: 39: 03.7$ & 7.18 & -107.9 & 18.65 & 0.34 & $121 \pm 29$ & $121 \pm 29$ & & 1 \\
\hline f08-36 & $12: 42: 37.77$ & $+02: 39: 09.6$ & 3.68 & -124.9 & 21.70 & 1.58 & $61 \pm 40$ & $80 \pm 54$ & f07-16 & 0 \\
\hline f08-38 & $12: 42: 31.35$ & $+02: 39: 28.2$ & 4.96 & -111.2 & 18.66 & 1.71 & $70 \pm 101$ & $70 \pm 101$ & & 1 \\
\hline f08-43 & $12: 42: 37.75$ & $+02: 39: 56.3$ & 3.31 & -113.7 & 21.69 & 1.82 & $-188 \pm 28$ & $-148 \pm 54$ & f07-24 & 0 \\
\hline f08-51 & $12: 42: 36.87$ & $+02: 40: 40.3$ & 3.30 & -100.4 & 20.73 & 2.88 & $-79 \pm 37$ & $-79 \pm 37$ & & 1 \\
\hline f08-55 & $12: 42: 23.94$ & $+02: 41: 02.9$ & 6.48 & -91.9 & 19.10 & 1.71 & $-71 \pm 30$ & $-42 \pm 41$ & f10-20 & 1 \\
\hline f08-57 & $12: 42: 37.47$ & $+02: 41: 11.6$ & 3.10 & -91.4 & 18.76 & 0.83 & $-2 \pm 27$ & $-2 \pm 27$ & & 1 \\
\hline f08-63 & $12: 42: 28.63$ & $+02: 41: 41.3$ & 5.32 & -85.5 & 18.55 & 2.27 & $-9 \pm 32$ & $-9 \pm 32$ & & 1 \\
\hline f08-64 & $12: 42: 35.25$ & $+02: 41: 45.8$ & 3.68 & -82.3 & 20.13 & 1.74 & $-71 \pm 15$ & $-18 \pm 31$ & f07-41 & 0 \\
\hline f09-01 & $12: 42: 16.50$ & $+02: 43: 53.2$ & 8.74 & -72.5 & 21.09 & 1.37 & $112 \pm 49$ & $106 \pm 66$ & f10-46 & 1 \\
\hline f09-05 & $12: 42: 24.76$ & $+02: 44: 20.0$ & 6.98 & -63.9 & 22.31 & 2.03 & $-118 \pm 78$ & $-57 \pm 87$ & f10-50 & 1 \\
\hline f09-08 & $12: 42: 22.03$ & $+02: 44: 49.7$ & 7.81 & -62.9 & 19.14 & 1.14 & $104 \pm 16$ & $164 \pm 31$ & f10-56 & 1 \\
\hline f09-13 & $12: 42: 28.90$ & $+02: 45: 21.4$ & 6.64 & -52.0 & 20.90 & 2.91 & $6 \pm 61$ & $-4 \pm 88$ & $3.2: 46$ & 1 \\
\hline f09-22 & $12: 42: 19.61$ & $+02: 46: 30.8$ & 9.20 & -55.2 & 21.13 & 3.19 & $150 \pm 73$ & $150 \pm 73$ & & 1 \\
\hline f09-24 & $12: 42: 24.89$ & $+02: 46: 59.4$ & 8.47 & -47.5 & 21.68 & 1.15 & $-38 \pm 34$ & $-38 \pm 34$ & & 1 \\
\hline f09-27 & $12: 42: 28.95$ & $+02: 47: 19.4$ & 8.00 & -40.8 & 19.92 & -0.13 & $43 \pm 34$ & $19 \pm 67$ & $3.2: 71$ & 1 \\
\hline f09-32 & $12: 42: 15.48$ & $+02: 47: 46.6$ & 10.78 & -52.8 & $\ldots$ & $\ldots$ & $73 \pm 16$ & $73 \pm 16$ & & 1 \\
\hline f09-35 & $12: 42: 21.02$ & $+02: 48: 01.9$ & 9.88 & -46.8 & $\ldots$ & $\ldots$ & $185 \pm 48$ & $185 \pm 48$ & & 1 \\
\hline f09-37 & $12: 42: 22.75$ & $+02: 48: 09.5$ & 9.66 & -44.5 & 20.78 & 0.58 & $41 \pm 43$ & $41 \pm 43$ & & 1 \\
\hline f09-38 & $12: 42: 28.92$ & $+02: 48: 16.8$ & 8.75 & -36.7 & 18.74 & 1.81 & $51 \pm 20$ & $17 \pm 43$ & $3.1: 88$ & 1 \\
\hline f09-41 & $12: 42: 26.36$ & $+02: 48: 34.7$ & 9.38 & -38.8 & 21.08 & 3.41 & $161 \pm 61$ & $81 \pm 118$ & $3.2: 89$ & 1 \\
\hline f09-43 & $12: 42: 22.01$ & $+02: 48: 45.5$ & 10.22 & -42.9 & 20.83 & 0.78 & $378 \pm 45$ & $378 \pm 45$ & & 1 \\
\hline f09-52 & $12: 42: 27.43$ & $+02: 49: 38.4$ & 10.08 & -33.8 & 20.39 & 1.11 & $22 \pm 29$ & $-8 \pm 40$ & $3.2: 109, \mathrm{f} 13-40$ & 1 \\
\hline f09-56 & $12: 42: 20.52$ & $+02: 49: 59.9$ & 11.40 & -40.0 & 21.62 & 0.64 & $-100 \pm 109$ & $-100 \pm 109$ & & 1 \\
\hline f09-57 & $12: 42: 20.78$ & $+02: 50: 08.6$ & 11.47 & -39.3 & 22.08 & 2.33 & $346 \pm 84$ & $346 \pm 84$ & & 1 \\
\hline f09-62 & $12: 42: 21.00$ & $+02: 50: 35.5$ & 11.79 & -37.7 & $\cdots$ & $\ldots$ & $72 \pm 35$ & $72 \pm 35$ & & 1 \\
\hline f10-02 & $12: 42: 28.82$ & $+02: 39: 04.7$ & 5.69 & -112.6 & 22.32 & 0.71 & $85 \pm 77$ & $85 \pm 77$ & & 1 \\
\hline f10-06 & $12: 42: 29.86$ & $+02: 39: 27.1$ & 5.32 & -110.0 & 21.90 & 1.40 & $372 \pm 46$ & $306 \pm 60$ & $2.2: 76$ & 1 \\
\hline f10-11 & $12: 42: 23.07$ & $+02: 40: 07.6$ & 6.79 & -99.7 & 22.33 & 0.62 & $67 \pm 81$ & $67 \pm 81$ & & 1 \\
\hline f10-12 & $12: 42: 26.86$ & $+02: 40: 16.2$ & 5.83 & -99.8 & 22.40 & 3.62 & $-4 \pm 205$ & $-4 \pm 205$ & & 1 \\
\hline f10-20 & $12: 42: 23.94$ & $+02: 41: 02.9$ & 6.48 & -91.9 & 19.10 & 1.71 & $-12 \pm 28$ & $-42 \pm 41$ & f08-55 & 0 \\
\hline f10-26 & $12: 42: 21.20$ & $+02: 41: 34.1$ & 7.17 & -87.6 & 18.91 & 1.11 & $-8 \pm 35$ & $-8 \pm 35$ & & 1 \\
\hline f10-34 & $12: 42: 28.15$ & $+02: 42: 41.7$ & 5.61 & -75.2 & 21.70 & 3.39 & $197 \pm 79$ & $197 \pm 79$ & & 1 \\
\hline f10-36 & $12: 42: 22.18$ & $+02: 42: 52.2$ & 7.10 & -76.9 & 18.61 & 2.74 & $58 \pm 40$ & $39 \pm 61$ & $3.2: 2$ & 1 \\
\hline f10-39 & $12: 42: 27.12$ & $+02: 43: 13.9$ & 6.01 & -70.9 & 18.53 & 0.00 & $266 \pm 37$ & $222 \pm 60$ & $3.1: 7$ & 1 \\
\hline f10-46 & $12: 42: 16.50$ & $+02: 43: 53.2$ & 8.74 & -72.5 & 21.09 & 1.37 & $100 \pm 45$ & $106 \pm 66$ & f09-01 & 0 \\
\hline
\end{tabular}


Y. Schuberth et al.: Dynamics of the NGC 4636 globular cluster system. II.

Table D.1. continued.

\begin{tabular}{|c|c|c|c|c|c|c|c|c|c|c|}
\hline ID & $\begin{array}{c}\text { RA } \\
(\mathrm{J} 2000)\end{array}$ & $\begin{array}{c}\text { Dec } \\
(\mathrm{J} 2000)\end{array}$ & $\begin{array}{c}R_{4636} \\
\text { [arcmin] }\end{array}$ & $\begin{array}{c}\text { PA } \\
{[\mathrm{deg}]}\end{array}$ & $\begin{array}{c}m_{R} \\
{[\mathrm{mag}]}\end{array}$ & $\begin{array}{l}C-R \\
{[\mathrm{mag}]}\end{array}$ & $\begin{array}{c}v_{\mathrm{spec}} \\
{\left[\mathrm{km} \mathrm{s}^{-1}\right]}\end{array}$ & $\begin{array}{c}v_{\text {final }} \\
{\left[\mathrm{km} \mathrm{s}^{-1}\right]}\end{array}$ & Cross-Identification(s) & $1 \mathrm{st}$ \\
\hline $\mathrm{f} 10-50$ & $12: 42: 24.76$ & $+02: 44: 20.0$ & 6.98 & -63.9 & 22.31 & 2.03 & $3 \pm 38$ & $-57 \pm 87$ & f09-05 & 0 \\
\hline f10-54 & $12: 42: 16.85$ & $+02: 44: 41.0$ & 8.93 & -67.5 & 19.49 & 1.37 & $75 \pm 60$ & $75 \pm 60$ & & 1 \\
\hline f10-56 & $12: 42: 22.03$ & $+02: 44: 49.7$ & 7.81 & -62.9 & 19.14 & 1.14 & $224 \pm 27$ & $164 \pm 31$ & f09-08 & 0 \\
\hline f10-59 & $12: 42: 28.49$ & $+02: 45: 03.1$ & 6.54 & -54.7 & 18.81 & 2.90 & $88 \pm 67$ & $88 \pm 67$ & & 1 \\
\hline f10-67 & $12: 42: 14.18$ & $+02: 45: 47.8$ & 10.00 & -63.1 & 18.87 & 1.44 & $165 \pm 23$ & $165 \pm 23$ & & 1 \\
\hline f12-01 & $12: 43: 21.54$ & $+02: 37: 17.5$ & 8.85 & 116.7 & 21.67 & 0.74 & $-90 \pm 60$ & $-90 \pm 60$ & & 1 \\
\hline f12-06 & $12: 43: 10.33$ & $+02: 37: 39.9$ & 6.25 & 125.2 & 22.05 & 0.67 & $-25 \pm 79$ & $22 \pm 98$ & f01-39 & 0 \\
\hline f12-10 & $12: 43: 09.42$ & $+02: 38: 05.5$ & 5.82 & 123.0 & 18.47 & -0.42 & $-48 \pm 24$ & $-48 \pm 24$ & & 1 \\
\hline f12-14 & $12: 43: 10.14$ & $+02: 38: 33.7$ & 5.74 & 118.1 & 19.45 & 0.82 & $-213 \pm 15$ & $-189 \pm 35$ & $6.1: 38$ & 1 \\
\hline f12-35 & $12: 43: 18.87$ & $+02: 41: 03.8$ & 7.24 & 91.6 & 18.37 & -1.45 & $-27 \pm 16$ & $-27 \pm 16$ & & 1 \\
\hline f12-37 & $12: 43: 21.84$ & $+02: 41: 19.4$ & 7.98 & 89.6 & 21.70 & 0.87 & $152 \pm 70$ & $152 \pm 70$ & & 1 \\
\hline f12-39 & $12: 43: 22.99$ & $+02: 41: 32.9$ & 8.28 & 88.0 & 18.34 & 1.60 & $-25 \pm 20$ & $-25 \pm 20$ & & 1 \\
\hline f12-46 & $12: 43: 09.33$ & $+02: 42: 21.7$ & 4.98 & 77.3 & 19.24 & 1.12 & $135 \pm 22$ & $112 \pm 31$ & $6.2: 102$ & 1 \\
\hline f12-47 & $12: 43: 24.13$ & $+02: 42: 27.5$ & 8.64 & 82.1 & 20.48 & 0.80 & $-260 \pm 40$ & $-260 \pm 40$ & & 1 \\
\hline f12-52 & $12: 43: 11.89$ & $+02: 43: 06.7$ & 5.80 & 71.5 & 18.49 & 0.87 & $50 \pm 22$ & $50 \pm 22$ & & 1 \\
\hline f12-54 & $12: 43: 09.37$ & $+02: 43: 18.9$ & 5.28 & 67.2 & & $\ldots$ & $6 \pm 33$ & $6 \pm 33$ & & 1 \\
\hline f12-60 & $12: 43: 13.75$ & $+02: 43: 56.3$ & 6.53 & 65.9 & 19.98 & 0.65 & $128 \pm 73$ & $128 \pm 73$ & & 1 \\
\hline f12-61 & $12: 43: 18.01$ & $+02: 44: 00.0$ & 7.54 & 68.7 & 20.43 & 0.78 & $138 \pm 47$ & $138 \pm 47$ & & 1 \\
\hline f13-14 & $12: 42: 41.91$ & $+02: 46: 52.1$ & 5.94 & -19.5 & 20.22 & 3.37 & $-24 \pm 46$ & $-24 \pm 46$ & & 1 \\
\hline f13-21 & $12: 42: 41.60$ & $+02: 47: 27.9$ & 6.53 & -18.4 & 20.43 & 3.40 & $-64 \pm 41$ & $-64 \pm 41$ & & 1 \\
\hline f13-28 & $12: 42: 31.57$ & $+02: 48: 08.8$ & 8.26 & -33.6 & 22.30 & 0.69 & $-122 \pm 58$ & $-122 \pm 58$ & & 1 \\
\hline f13-29 & $12: 42: 41.39$ & $+02: 48: 13.8$ & 7.28 & -16.9 & 21.72 & 3.39 & $-29 \pm 70$ & $-29 \pm 70$ & & 1 \\
\hline f13-35 & $12: 42: 31.76$ & $+02: 49: 05.4$ & 9.04 & -30.0 & 21.88 & 1.69 & $2 \pm 46$ & $2 \pm 46$ & & 1 \\
\hline f13-39 & $12: 42: 32.75$ & $+02: 49: 29.6$ & 9.27 & -27.5 & 18.86 & 0.88 & $-130 \pm 18$ & $-132 \pm 29$ & $3.1: 107$ & 1 \\
\hline f13-40 & $12: 42: 27.43$ & $+02: 49: 38.4$ & 10.08 & -33.8 & 20.39 & 1.11 & $-20 \pm 15$ & $-8 \pm 40$ & $3.2: 109$, f09-52 & 0 \\
\hline f13-42 & $12: 42: 37.39$ & $+02: 49: 51.0$ & 9.13 & -20.0 & 22.11 & 1.80 & $-21 \pm 86$ & $-21 \pm 86$ & & 1 \\
\hline f13-43 & $12: 42: 36.48$ & $+02: 49: 54.9$ & 9.27 & -21.1 & 21.31 & 1.08 & $243 \pm 31$ & $243 \pm 31$ & & 1 \\
\hline f13-48 & $12: 42: 40.69$ & $+02: 50: 23.9$ & 9.42 & -14.1 & 21.35 & 1.05 & $48 \pm 22$ & $22 \pm 44$ & f04-43 & 0 \\
\hline f13-49 & $12: 42: 38.49$ & $+02: 50: 31.6$ & 9.69 & -17.1 & 20.48 & 1.36 & $90 \pm 20$ & $74 \pm 28$ & f04-44 & 0 \\
\hline f13-55 & $12: 42: 42.15$ & $+02: 51: 21.4$ & 10.27 & -10.8 & 21.52 & 3.64 & $156 \pm 67$ & $156 \pm 67$ & & 1 \\
\hline f13-58 & $12: 42: 27.88$ & $+02: 51: 46.1$ & 11.85 & -27.6 & 22.12 & 0.75 & $63 \pm 65$ & $63 \pm 65$ & & 1 \\
\hline f13-59 & $12: 42: 43.44$ & $+02: 51: 49.7$ & 10.68 & -8.6 & 19.07 & 1.10 & $17 \pm 17$ & $27 \pm 22$ & f04-57 & 0 \\
\hline f13-60 & $12: 42: 32.91$ & $+02: 51: 55.0$ & 11.46 & -21.7 & 21.35 & 0.92 & $-2 \pm 50$ & $-2 \pm 50$ & & 1 \\
\hline f13-65 & $12: 42: 37.55$ & $+02: 52: 20.7$ & 11.50 & -15.5 & 22.05 & 0.79 & $51 \pm 61$ & $27 \pm 86$ & f13-66 & 1 \\
\hline f13-66 & $12: 42: 37.55$ & $+02: 52: 20.7$ & 11.50 & -15.5 & 22.05 & 0.79 & $3 \pm 61$ & $27 \pm 86$ & f13-65 & 0 \\
\hline f13-68 & $12: 42: 30.67$ & $+02: 52: 34.7$ & 12.29 & -23.0 & 22.19 & 0.78 & $-36 \pm 66$ & $-36 \pm 66$ & & 1 \\
\hline $1.1: 4$ & $12: 42: 44.29$ & $+02: 36: 05.8$ & 5.35 & -164.9 & 20.87 & 3.31 & $-119 \pm 50$ & $-119 \pm 50$ & & 1 \\
\hline $1.1: 22$ & $12: 42: 52.90$ & $+02: 37: 19.5$ & 4.01 & 169.1 & 21.33 & 1.29 & $178 \pm 77$ & $215 \pm 83$ & f06-51 & 0 \\
\hline $1.1: 58$ & $12: 42: 52.67$ & $+02: 39: 44.2$ & 1.68 & 155.4 & 20.55 & 0.99 & $-131 \pm 22$ & $-131 \pm 22$ & & 1 \\
\hline $1.1: 105$ & $12: 42: 48.74$ & $+02: 42: 28.7$ & 1.24 & -13.1 & 18.93 & 0.50 & $270 \pm 45$ & $270 \pm 45$ & & 1 \\
\hline $1.2: 10$ & $12: 42: 40.32$ & $+02: 36: 28.7$ & 5.35 & -153.5 & 20.06 & 0.78 & $199 \pm 154$ & $199 \pm 154$ & & 1 \\
\hline $1.2: 14$ & $12: 42: 51.96$ & $+02: 36: 43.1$ & 4.58 & 173.5 & 19.25 & 0.90 & $166 \pm 21$ & $166 \pm 21$ & & 1 \\
\hline $1.2: 24$ & $12: 42: 41.45$ & $+02: 37: 28.1$ & 4.34 & -151.0 & 20.60 & 0.98 & $56 \pm 70$ & $56 \pm 70$ & & 1 \\
\hline $1.2: 30$ & $12: 42: 50.55$ & $+02: 37: 50.5$ & 3.43 & 177.2 & 21.89 & 1.93 & $229 \pm 38$ & $250 \pm 47$ & $1.3: 33$ & 1 \\
\hline $1.3: 7$ & $12: 42: 52.55$ & $+02: 36: 16.6$ & 5.03 & 172.4 & 21.21 & 2.45 & $126 \pm 54$ & $126 \pm 54$ & & 1 \\
\hline $1.3: 11$ & $12: 42: 53.93$ & $+02: 36: 30.4$ & 4.87 & 168.0 & 19.94 & 1.29 & $89 \pm 48$ & $89 \pm 48$ & & 1 \\
\hline $1.3: 15$ & $12: 42: 48.28$ & $+02: 36: 44.5$ & 4.54 & -175.0 & 21.11 & 0.86 & $-181 \pm 52$ & $-181 \pm 52$ & & 1 \\
\hline $1.3: 26$ & $12: 42: 50.86$ & $+02: 37: 26.5$ & 3.83 & 176.3 & 21.69 & 2.33 & $252 \pm 27$ & $252 \pm 27$ & & 1 \\
\hline $1.3: 29$ & $12: 42: 58.24$ & $+02: 37: 36.0$ & 4.22 & 150.3 & 20.58 & 2.72 & $-84 \pm 51$ & $-84 \pm 51$ & & 1 \\
\hline $1.3: 33$ & $12: 42: 50.55$ & $+02: 37: 50.5$ & 3.43 & 177.2 & 21.89 & 1.93 & $272 \pm 27$ & $250 \pm 47$ & $1.2: 30$ & 0 \\
\hline $1.3: 37$ & $12: 42: 57.94$ & $+02: 38: 03.4$ & 3.79 & 147.9 & 20.67 & 0.82 & $-120 \pm 32$ & $-120 \pm 32$ & & 1 \\
\hline $1.3: 39$ & $12: 42: 44.93$ & $+02: 38: 12.9$ & 3.29 & -158.0 & 19.99 & 3.01 & $-58 \pm 52$ & $-58 \pm 52$ & & 1 \\
\hline $1.3: 91$ & $12: 42: 58.61$ & $+02: 41: 52.3$ & 2.26 & 74.5 & 18.93 & 0.55 & $93 \pm 88$ & $71 \pm 108$ & f02-46 & 0 \\
\hline $1.3: 105$ & $12: 42: 48.92$ & $+02: 42: 43.9$ & 1.48 & -9.2 & 18.98 & 0.30 & $58 \pm 66$ & $58 \pm 66$ & & 1 \\
\hline $2.1: 3$ & $12: 42: 21.82$ & $+02: 34: 04.1$ & 10.04 & -135.8 & 21.23 & 1.79 & $215 \pm 51$ & $215 \pm 51$ & & 1 \\
\hline $2.1: 5$ & $12: 42: 24.43$ & $+02: 34: 15.4$ & 9.46 & -137.8 & 19.56 & 0.75 & $136 \pm 28$ & $136 \pm 28$ & & 1 \\
\hline $2.1: 9$ & $12: 42: 19.16$ & $+02: 34: 34.1$ & 10.18 & -131.1 & 20.17 & 3.58 & $-34 \pm 66$ & $-34 \pm 66$ & & 1 \\
\hline $2.1: 13$ & $12: 42: 19.35$ & $+02: 34: 48.5$ & 9.99 & -130.3 & 22.14 & 3.08 & $198 \pm 129$ & $198 \pm 129$ & & 1 \\
\hline $2.1: 16$ & $12: 42: 23.69$ & $+02: 35: 01.1$ & 9.04 & -133.7 & 21.68 & 3.53 & $-137 \pm 46$ & $-137 \pm 46$ & & 1 \\
\hline $2.1: 18$ & $12: 42: 15.61$ & $+02: 35: 08.4$ & 10.52 & -125.6 & 20.85 & 0.89 & $-87 \pm 26$ & $-87 \pm 26$ & & 1 \\
\hline $2.1: 19$ & $12: 42: 11.82$ & $+02: 35: 12.4$ & 11.27 & -122.5 & 21.63 & 0.59 & $263 \pm 81$ & $263 \pm 81$ & & 1 \\
\hline $2.1: 36$ & $12: 42: 20.93$ & $+02: 36: 26.9$ & 8.69 & -123.7 & 20.55 & 2.91 & $38 \pm 52$ & $38 \pm 52$ & & 1 \\
\hline $2.1: 40$ & $12: 42: 20.16$ & $+02: 36: 38.8$ & 8.74 & -121.9 & 20.70 & 0.96 & $17 \pm 37$ & $17 \pm 37$ & & 1 \\
\hline $2.1: 51$ & $12: 42: 18.12$ & $+02: 37: 40.2$ & 8.71 & -114.4 & 19.82 & 1.09 & $-29 \pm 27$ & $-29 \pm 27$ & & 1 \\
\hline $2.1: 56$ & $12: 42: 14.04$ & $+02: 38: 04.7$ & 9.50 & -109.6 & 22.06 & 2.53 & $97 \pm 72$ & $97 \pm 72$ & & 1 \\
\hline $2.1: 60$ & $12: 42: 20.32$ & $+02: 38: 15.8$ & 7.97 & -112.1 & 20.41 & 3.66 & $43 \pm 60$ & $43 \pm 60$ & & 1 \\
\hline
\end{tabular}


Table D.1. continued.

\begin{tabular}{|c|c|c|c|c|c|c|c|c|c|c|}
\hline ID & $\begin{array}{c}\text { RA } \\
(\mathrm{J} 2000)\end{array}$ & $\begin{array}{c}\text { Dec } \\
(\mathrm{J} 2000)\end{array}$ & $\begin{array}{c}R_{4636} \\
\text { [arcmin] }\end{array}$ & $\begin{array}{c}\text { PA } \\
{[\mathrm{deg}]}\end{array}$ & $\begin{array}{c}m_{R} \\
{[\mathrm{mag}]}\end{array}$ & $\begin{array}{l}C-R \\
{[\mathrm{mag}]}\end{array}$ & $\begin{array}{c}v_{\text {spec }} \\
{\left[\mathrm{km} \mathrm{s}^{-1}\right]}\end{array}$ & $\begin{array}{c}v_{\text {final }} \\
{\left[\mathrm{km} \mathrm{s}^{-1}\right]}\end{array}$ & Cross-Identification(s) & $1 \mathrm{st}$ \\
\hline $2.1: 61$ & $12: 42: 16.30$ & $+02: 38: 18.1$ & 8.89 & -109.5 & 21.06 & 0.86 & $-40 \pm 52$ & $-40 \pm 52$ & & 1 \\
\hline $2.1: 62$ & $12: 42: 22.54$ & $+02: 38: 22.8$ & 7.41 & -112.9 & 20.85 & 3.18 & $-146 \pm 67$ & $-146 \pm 67$ & & 1 \\
\hline 2.1:64 & $12: 42: 11.24$ & $+02: 38: 30.2$ & 10.03 & -106.0 & 22.13 & 3.16 & $4 \pm 60$ & $4 \pm 60$ & & 1 \\
\hline $2.1: 65$ & $12: 42: 18.90$ & $+02: 38: 34.5$ & 8.19 & -109.2 & 19.96 & 1.27 & $-52 \pm 27$ & $-52 \pm 27$ & & 1 \\
\hline 2.1:67 & $12: 42: 24.58$ & $+02: 38: 42.3$ & 6.82 & -112.1 & 22.09 & 2.19 & $-132 \pm 51$ & $-132 \pm 51$ & & 1 \\
\hline 2.1:69 & $12: 42: 18.25$ & $+02: 38: 52.3$ & 8.25 & -106.9 & 18.35 & -0.78 & $-88 \pm 18$ & $-88 \pm 18$ & & 1 \\
\hline $2.1: 71$ & $12: 42: 17.77$ & $+02: 39: 01.1$ & 8.33 & -105.7 & 18.54 & 1.76 & $-15 \pm 36$ & $-15 \pm 36$ & & 1 \\
\hline $2.1: 82$ & $12: 42: 22.07$ & $+02: 39: 39.4$ & 7.13 & -103.1 & 20.68 & 0.72 & $150 \pm 39$ & $150 \pm 39$ & & 1 \\
\hline $2.1: 85$ & $12: 42: 13.56$ & $+02: 39: 53.6$ & 9.17 & -98.6 & 21.69 & 1.52 & $250 \pm 61$ & $250 \pm 61$ & & 1 \\
\hline $2.1: 87$ & $12: 42: 19.92$ & $+02: 40: 01.2$ & 7.58 & -99.5 & 21.73 & 1.42 & $217 \pm 140$ & $217 \pm 140$ & & 1 \\
\hline $2.2: 5$ & $12: 42: 27.20$ & $+02: 34: 17.1$ & 8.99 & -141.0 & 20.16 & 3.64 & $-74 \pm 57$ & $-74 \pm 57$ & & 1 \\
\hline $2.2: 16$ & $12: 42: 22.19$ & $+02: 35: 09.6$ & 9.22 & -131.5 & 20.64 & 3.66 & $-121 \pm 187$ & $-121 \pm 187$ & & 1 \\
\hline $2.2: 26$ & $12: 42: 28.48$ & $+02: 35: 50.0$ & 7.62 & -135.5 & 21.05 & 2.32 & $-170 \pm 45$ & $-108 \pm 74$ & f08-09 & 0 \\
\hline $2.2: 27$ & $12: 42: 21.60$ & $+02: 35: 53.8$ & 8.87 & -127.3 & 20.39 & 2.76 & $-110 \pm 55$ & $-110 \pm 55$ & & 1 \\
\hline $2.2: 35$ & $12: 42: 30.11$ & $+02: 36: 23.6$ & 6.94 & -134.6 & 19.88 & 1.32 & $-138 \pm 45$ & $-138 \pm 45$ & & 1 \\
\hline $2.2: 50$ & $12: 42: 16.37$ & $+02: 37: 34.8$ & 9.14 & -113.8 & 19.08 & 0.93 & $-42 \pm 40$ & $-42 \pm 40$ & & 1 \\
\hline $2.2: 53$ & $12: 42: 19.87$ & $+02: 37: 48.9$ & 8.25 & -114.7 & 19.80 & 4.30 & $-48 \pm 85$ & $-48 \pm 85$ & & 1 \\
\hline $2.2: 55$ & $12: 42: 18.69$ & $+02: 38: 02.2$ & 8.43 & -112.5 & 18.49 & 0.54 & $-67 \pm 26$ & $-67 \pm 26$ & & 1 \\
\hline $2.2: 66$ & $12: 42: 29.58$ & $+02: 38: 46.8$ & 5.64 & -116.1 & 21.15 & 3.08 & $-188 \pm 65$ & $-188 \pm 65$ & & 1 \\
\hline $2.2: 68$ & $12: 42: 21.32$ & $+02: 38: 54.0$ & 7.51 & -108.4 & 20.17 & 1.14 & $237 \pm 22$ & $237 \pm 22$ & & 1 \\
\hline $2.2: 75$ & $12: 42: 28.04$ & $+02: 39: 23.6$ & 5.76 & -109.0 & 19.97 & 3.36 & $-31 \pm 47$ & $-31 \pm 47$ & & 1 \\
\hline $2.2: 76$ & $12: 42: 29.86$ & $+02: 39: 27.1$ & 5.32 & -110.0 & 21.90 & 1.40 & $240 \pm 39$ & $306 \pm 60$ & f10-06 & 0 \\
\hline $2.2: 78$ & $12: 42: 29.35$ & $+02: 39: 35.7$ & 5.39 & -108.1 & 18.64 & 0.33 & $-97 \pm 28$ & $-97 \pm 28$ & & 1 \\
\hline $2.2: 89$ & $12: 42: 23.99$ & $+02: 40: 17.6$ & 6.54 & -98.6 & 21.69 & 0.75 & $-236 \pm 38$ & $-236 \pm 38$ & & 1 \\
\hline 2.2:95 & $12: 42: 31.08$ & $+02: 40: 36.4$ & 4.74 & -98.0 & 20.36 & 2.52 & $34 \pm 42$ & $34 \pm 42$ & & 1 \\
\hline 2.2:96 & $12: 42: 29.83$ & $+02: 40: 40.3$ & 5.04 & -96.8 & 20.61 & 3.24 & $-17 \pm 44$ & $-17 \pm 44$ & & 1 \\
\hline 2.2:97 & $12: 42: 29.01$ & $+02: 40: 44.1$ & 5.24 & -95.8 & 20.98 & 1.78 & $9 \pm 30$ & $9 \pm 30$ & & 1 \\
\hline $3.1: 7$ & $12: 42: 27.12$ & $+02: 43: 13.9$ & 6.01 & -70.9 & 18.53 & 0.00 & $178 \pm 48$ & $222 \pm 60$ & f10-39 & 0 \\
\hline $3.1: 20$ & $12: 42: 34.62$ & $+02: 44: 02.3$ & 4.71 & -54.0 & 19.19 & 1.70 & $1 \pm 43$ & $41 \pm 46$ & f07-67 & 0 \\
\hline $3.1: 27$ & $12: 42: 41.93$ & $+02: 44: 25.9$ & 3.73 & -32.1 & 18.31 & 1.80 & $-51 \pm 38$ & $-51 \pm 38$ & & 1 \\
\hline $3.1: 42$ & $12: 42: 31.26$ & $+02: 45: 18.7$ & 6.16 & -49.0 & 20.10 & 1.93 & $-4 \pm 40$ & $-4 \pm 40$ & & 1 \\
\hline $3.1: 49$ & $12: 42: 33.86$ & $+02: 45: 39.9$ & 5.94 & -42.3 & 21.98 & 1.67 & $-47 \pm 54$ & $-47 \pm 54$ & & 1 \\
\hline $3.1: 60$ & $12: 42: 29.22$ & $+02: 46: 31.7$ & 7.37 & -44.4 & 19.47 & 2.56 & $-97 \pm 47$ & $-97 \pm 47$ & & 1 \\
\hline $3.1: 87$ & $12: 42: 33.21$ & $+02: 48: 10.9$ & 8.07 & -31.0 & 20.21 & 2.77 & $-30 \pm 51$ & $-36 \pm 72$ & $3.2: 82$ & 1 \\
\hline $3.1: 88$ & $12: 42: 28.92$ & $+02: 48: 16.8$ & 8.75 & -36.7 & 18.74 & 1.81 & $-17 \pm 38$ & $17 \pm 43$ & f09-38 & 0 \\
\hline $3.1: 97$ & $12: 42: 36.77$ & $+02: 48: 51.1$ & 8.26 & -23.3 & 22.09 & 1.84 & $-163 \pm 32$ & $-163 \pm 32$ & & 1 \\
\hline 3.1:107 & $12: 42: 32.75$ & $+02: 49: 29.6$ & 9.27 & -27.5 & 18.86 & 0.88 & $-134 \pm 22$ & $-132 \pm 29$ & f13-39 & 0 \\
\hline $3.2: 2$ & $12: 42: 22.18$ & $+02: 42: 52.2$ & 7.10 & -76.9 & 18.61 & 2.74 & $20 \pm 46$ & $39 \pm 61$ & f10-36 & 0 \\
\hline $3.2: 17$ & $12: 42: 25.06$ & $+02: 43: 47.8$ & 6.69 & -67.8 & 20.68 & 3.29 & $-69 \pm 58$ & $-69 \pm 58$ & & 1 \\
\hline $3.2: 21$ & $12: 42: 36.98$ & $+02: 44: 02.4$ & 4.25 & -49.3 & 21.41 & 3.36 & $-2 \pm 46$ & $-2 \pm 46$ & & 1 \\
\hline $3.2: 26$ & $12: 42: 37.20$ & $+02: 44: 16.7$ & 4.37 & -46.4 & 20.52 & 3.19 & $19 \pm 58$ & $19 \pm 58$ & & 1 \\
\hline $3.2: 27$ & $12: 42: 34.38$ & $+02: 44: 19.9$ & 4.94 & -51.6 & 20.42 & 3.55 & $47 \pm 56$ & $47 \pm 56$ & & 1 \\
\hline $3.2: 40$ & $12: 42: 23.10$ & $+02: 45: 03.8$ & 7.69 & -60.4 & 21.63 & 2.72 & $-3 \pm 72$ & $-3 \pm 72$ & & 1 \\
\hline $3.2: 46$ & $12: 42: 28.90$ & $+02: 45: 21.4$ & 6.64 & -52.0 & 20.90 & 2.91 & $-14 \pm 64$ & $-4 \pm 88$ & f09-13 & 0 \\
\hline $3.2: 66$ & $12: 42: 30.27$ & $+02: 46: 59.0$ & 7.53 & -40.6 & 21.80 & 2.89 & $63 \pm 60$ & $63 \pm 60$ & & 1 \\
\hline $3.2: 71$ & $12: 42: 28.95$ & $+02: 47: 19.4$ & 8.00 & -40.8 & 19.92 & -0.13 & $-5 \pm 58$ & $19 \pm 67$ & f09-27 & 0 \\
\hline $3.2: 75$ & $12: 42: 21.53$ & $+02: 47: 40.0$ & 9.54 & -47.9 & 22.22 & 0.99 & $-18 \pm 44$ & $-18 \pm 44$ & & 1 \\
\hline $3.2: 82$ & $12: 42: 33.21$ & $+02: 48: 10.9$ & 8.07 & -31.0 & 20.21 & 2.77 & $-42 \pm 51$ & $-36 \pm 72$ & $3.1: 87$ & 0 \\
\hline $3.2: 89$ & $12: 42: 26.36$ & $+02: 48: 34.7$ & 9.38 & -38.8 & 21.08 & 3.41 & $0 \pm 101$ & $81 \pm 118$ & f09-41 & 0 \\
\hline $3.2: 92$ & $12: 42: 37.45$ & $+02: 48: 43.0$ & 8.07 & -22.6 & 19.36 & 3.32 & $-56 \pm 41$ & $-56 \pm 41$ & & 1 \\
\hline $3.2: 109$ & $12: 42: 27.43$ & $+02: 49: 38.4$ & 10.08 & -33.8 & 20.39 & 1.11 & $-25 \pm 23$ & $-8 \pm 40$ & f09-52, f13-40 & 0 \\
\hline $4.1: 5$ & $12: 42: 32.99$ & $+02: 50: 14.3$ & 9.91 & -25.2 & 20.59 & 2.43 & $-5 \pm 46$ & $-5 \pm 46$ & & 1 \\
\hline $4.1: 11$ & $12: 42: 35.60$ & $+02: 50: 31.9$ & 9.93 & -21.0 & 21.43 & 0.68 & $-5 \pm 53$ & $-5 \pm 53$ & & 1 \\
\hline $4.1: 37$ & $12: 42: 25.94$ & $+02: 52: 22.0$ & 12.61 & -28.3 & 21.37 & 0.64 & $-180 \pm 47$ & $-180 \pm 47$ & & 1 \\
\hline $4.1: 38$ & $12: 42: 31.44$ & $+02: 52: 24.5$ & 12.05 & -22.4 & 20.71 & 0.58 & $142 \pm 62$ & $142 \pm 62$ & & 1 \\
\hline 4.1:42 & $12: 42: 29.92$ & $+02: 52: 41.2$ & 12.46 & -23.6 & 18.85 & 0.47 & $192 \pm 23$ & $192 \pm 23$ & & 1 \\
\hline 4.1:46 & $12: 42: 29.31$ & $+02: 53: 11.5$ & 12.98 & -23.3 & 20.88 & 0.96 & $60 \pm 59$ & $60 \pm 59$ & & 1 \\
\hline 4.1:49 & $12: 42: 25.09$ & $+02: 53: 23.4$ & 13.61 & -27.0 & 20.37 & 2.94 & $23 \pm 48$ & $23 \pm 48$ & & 1 \\
\hline $4.1: 52$ & $12: 42: 34.49$ & $+02: 53: 34.0$ & 12.89 & -17.3 & 20.66 & 0.77 & $85 \pm 34$ & $85 \pm 34$ & & 1 \\
\hline $4.1: 56$ & $12: 42: 23.64$ & $+02: 53: 49.1$ & 14.16 & -27.6 & 21.74 & 3.56 & $-102 \pm 48$ & $-102 \pm 48$ & & 1 \\
\hline $4.1: 58$ & $12: 42: 34.75$ & $+02: 53: 58.1$ & 13.25 & -16.6 & 18.81 & 1.68 & $-92 \pm 75$ & $-92 \pm 75$ & & 1 \\
\hline $4.1: 60$ & $12: 42: 28.19$ & $+02: 54: 05.5$ & 13.92 & -22.9 & 20.67 & 3.67 & $113 \pm 51$ & $113 \pm 51$ & & 1 \\
\hline $4.1: 65$ & $12: 42: 25.97$ & $+02: 54: 24.2$ & 14.43 & -24.4 & 21.10 & 3.18 & $-37 \pm 46$ & $-37 \pm 46$ & & 1 \\
\hline $4.1: 69$ & $12: 42: 24.71$ & $+02: 54: 44.7$ & 14.87 & -25.0 & 18.66 & 2.41 & $83 \pm 52$ & $83 \pm 52$ & & 1 \\
\hline 4.1:74 & $12: 42: 28.16$ & $+02: 55: 07.5$ & 14.88 & -21.4 & 20.42 & 3.15 & $-16 \pm 53$ & $-16 \pm 53$ & & 1 \\
\hline 4.1:77 & $12: 42: 29.12$ & $+02: 55: 25.5$ & 15.08 & -20.1 & 19.50 & 3.40 & $-61 \pm 44$ & $-61 \pm 44$ & & 1 \\
\hline
\end{tabular}


Y. Schuberth et al.: Dynamics of the NGC 4636 globular cluster system. II.

Table D.1. continued.

\begin{tabular}{|c|c|c|c|c|c|c|c|c|c|c|}
\hline ID & $\begin{array}{c}\mathrm{RA} \\
(\mathrm{J} 2000)\end{array}$ & $\begin{array}{c}\text { Dec } \\
(\mathrm{J} 2000)\end{array}$ & $\begin{array}{c}R_{4636} \\
{[\operatorname{arcmin]}]}\end{array}$ & $\begin{array}{c}\text { PA } \\
{[\mathrm{deg}]}\end{array}$ & $\begin{array}{c}m_{R} \\
{[\mathrm{mag}]}\end{array}$ & $\begin{array}{l}C-R \\
{[\mathrm{mag}]}\end{array}$ & $\begin{array}{c}v_{\mathrm{spec}} \\
{\left[\mathrm{km} \mathrm{s}^{-1}\right]}\end{array}$ & $\begin{array}{c}v_{\text {final }} \\
{\left[\mathrm{km} \mathrm{s}^{-1}\right]}\end{array}$ & Cross-Identification(s) & $1 \mathrm{st}$ \\
\hline $4.1: 86$ & $12: 42: 35.13$ & $+02: 56: 03.8$ & 15.25 & -14.0 & 20.40 & 2.45 & $-82 \pm 56$ & $-82 \pm 56$ & & 1 \\
\hline $4.1: 88$ & $12: 42: 28.50$ & $+02: 56: 16.6$ & 15.93 & -19.6 & 20.81 & 1.52 & $224 \pm 32$ & $224 \pm 32$ & & 1 \\
\hline 4.1:90 & $12: 42: 27.43$ & $+02: 56: 22.4$ & 16.11 & -20.3 & 21.16 & 3.81 & $-103 \pm 41$ & $-103 \pm 41$ & & 1 \\
\hline $4.1: 97$ & $12: 42: 30.92$ & $+02: 56: 46.0$ & 16.21 & -17.0 & 18.43 & 0.90 & $20 \pm 22$ & $20 \pm 22$ & & 1 \\
\hline $5.1: 4$ & $12: 42: 58.16$ & $+02: 43: 31.9$ & 3.07 & 42.4 & 21.21 & 3.01 & $133 \pm 26$ & $133 \pm 26$ & & 1 \\
\hline $5.1: 11$ & $12: 42: 55.57$ & $+02: 43: 53.1$ & 2.98 & 28.5 & 18.44 & 1.47 & $19 \pm 29$ & $19 \pm 29$ & & 1 \\
\hline $5.1: 13$ & $12: 43: 05.04$ & $+02: 44: 03.4$ & 4.70 & 53.6 & 18.88 & 2.27 & $-70 \pm 44$ & $-89 \pm 54$ & f02-67 & 0 \\
\hline $5.1: 14$ & $12: 42: 57.95$ & $+02: 44: 09.5$ & 3.53 & 34.9 & 21.71 & 2.98 & $70 \pm 34$ & $70 \pm 34$ & & 1 \\
\hline $5.1: 42$ & 12:43:02.91 & $+02: 45: 45.0$ & 5.54 & 36.0 & 21.35 & 3.05 & $24 \pm 51$ & $24 \pm 51$ & & 1 \\
\hline $5.1: 51$ & $12: 43: 07.15$ & $+02: 46: 45.7$ & 6.99 & 38.1 & 20.07 & 3.79 & $-22 \pm 55$ & $-22 \pm 55$ & & 1 \\
\hline $5.1: 57$ & $12: 42: 58.92$ & $+02: 47: 08.1$ & 6.29 & 21.1 & 21.19 & 0.87 & $35 \pm 30$ & $35 \pm 30$ & & 1 \\
\hline $5.1: 75$ & $12: 43: 00.47$ & $+02: 48: 15.3$ & 7.47 & 20.7 & 20.06 & 2.72 & $13 \pm 49$ & $17 \pm 93$ & f03-45 & 0 \\
\hline $5.1: 77$ & $12: 43: 09.11$ & $+02: 48: 28.0$ & 8.66 & 33.7 & 18.24 & -0.19 & $129 \pm 21$ & $108 \pm 65$ & f03-47 & 0 \\
\hline $5.1: 81$ & $12: 42: 54.00$ & $+02: 48: 46.6$ & 7.58 & 7.8 & 22.26 & 0.98 & $170 \pm 52$ & $170 \pm 52$ & & 1 \\
\hline $5.1: 88$ & $12: 42: 53.65$ & $+02: 49: 09.4$ & 7.95 & 6.8 & 19.68 & 3.59 & $-57 \pm 42$ & $-57 \pm 42$ & & 1 \\
\hline $5.1: 96$ & $12: 43: 05.91$ & $+02: 49: 41.8$ & 9.33 & 25.4 & 19.82 & 3.14 & $-38 \pm 51$ & $-38 \pm 51$ & & 1 \\
\hline $5.1: 99$ & $12: 42: 57.99$ & $+02: 49: 52.2$ & 8.84 & 13.3 & 20.07 & 0.54 & $103 \pm 52$ & $103 \pm 52$ & & 1 \\
\hline $5.1: 104$ & $12: 42: 59.57$ & $+02: 50: 12.9$ & 9.27 & 15.1 & 21.53 & 0.77 & $36 \pm 65$ & $36 \pm 65$ & & 1 \\
\hline $6.1: 2$ & $12: 43: 07.52$ & $+02: 36: 03.7$ & 6.82 & 139.7 & 21.60 & 2.56 & $203 \pm 43$ & $203 \pm 43$ & & 1 \\
\hline $6.1: 5$ & $12: 43: 21.31$ & $+02: 36: 14.5$ & 9.32 & 122.6 & 19.62 & 3.35 & $-32 \pm 38$ & $-32 \pm 38$ & & 1 \\
\hline $6.1: 13$ & $12: 43: 22.41$ & $+02: 36: 49.6$ & 9.26 & 118.6 & 19.49 & 0.89 & $-166 \pm 60$ & $-166 \pm 60$ & & 1 \\
\hline $6.1: 18$ & $12: 43: 23.80$ & $+02: 37: 08.3$ & 9.43 & 116.0 & 21.37 & 3.09 & $-51 \pm 46$ & $-51 \pm 46$ & & 1 \\
\hline $6.1: 22$ & $12: 43: 08.03$ & $+02: 37: 27.6$ & 5.92 & 130.0 & 19.32 & 3.34 & $91 \pm 60$ & $91 \pm 60$ & & 1 \\
\hline $6.1: 35$ & $12: 43: 17.86$ & $+02: 38: 21.7$ & 7.57 & 112.6 & 21.03 & 3.38 & $-91 \pm 77$ & $-91 \pm 77$ & & 1 \\
\hline $6.1: 37$ & $12: 43: 20.65$ & $+02: 38: 28.2$ & 8.18 & 110.0 & 18.34 & 0.99 & $36 \pm 35$ & $36 \pm 35$ & & 1 \\
\hline $6.1: 38$ & $12: 43: 10.14$ & $+02: 38: 33.7$ & 5.74 & 118.1 & 19.45 & 0.82 & $-166 \pm 32$ & $-189 \pm 35$ & f12-14 & 0 \\
\hline $6.1: 42$ & $12: 43: 12.60$ & $+02: 38: 45.8$ & 6.20 & 113.8 & 20.17 & 0.56 & $-30 \pm 81$ & $-80 \pm 93$ & $6.2: 50$ & 1 \\
\hline $6.1: 50$ & $12: 43: 08.48$ & $+02: 39: 24.9$ & 5.00 & 111.7 & 20.21 & 0.68 & $-177 \pm 38$ & $-177 \pm 38$ & & 1 \\
\hline $6.1: 53$ & $12: 43: 12.96$ & $+02: 39: 35.5$ & 6.00 & 106.2 & 20.47 & 1.14 & $-72 \pm 64$ & $-72 \pm 64$ & & 1 \\
\hline $6.1: 62$ & $12: 43: 07.91$ & $+02: 40: 06.8$ & 4.65 & 104.4 & 21.00 & 0.79 & $-179 \pm 61$ & $-179 \pm 61$ & & 1 \\
\hline $6.1: 75$ & $12: 43: 21.70$ & $+02: 41: 04.1$ & 7.95 & 91.4 & 21.31 & 3.06 & $-108 \pm 62$ & $-108 \pm 62$ & & 1 \\
\hline $6.1: 86$ & 12:43:16.71 & $+02: 41: 48.0$ & 6.72 & 85.4 & 21.13 & 3.33 & $53 \pm 72$ & $53 \pm 72$ & & 1 \\
\hline $6.1: 87$ & $12: 43: 13.14$ & $+02: 41: 51.3$ & 5.84 & 84.2 & 20.53 & 3.32 & $-16 \pm 43$ & $-16 \pm 43$ & & 1 \\
\hline $6.1: 99$ & $12: 43: 09.88$ & $+02: 42: 30.8$ & 5.15 & 76.0 & 21.52 & 3.93 & $-77 \pm 37$ & $-77 \pm 37$ & & 1 \\
\hline $6.1: 100$ & $12: 43: 14.80$ & $+02: 42: 33.8$ & 6.36 & 78.2 & 22.00 & 3.26 & $-248 \pm 28$ & $-248 \pm 28$ & & 1 \\
\hline $6.2: 6$ & $12: 43: 17.11$ & $+02: 36: 14.9$ & 8.45 & 126.4 & 21.89 & 0.73 & $3 \pm 157$ & $3 \pm 157$ & & 1 \\
\hline $6.2: 7$ & $12: 43: 14.85$ & $+02: 36: 19.7$ & 7.96 & 128.4 & 21.81 & 1.01 & $-120 \pm 19$ & $-120 \pm 19$ & & 1 \\
\hline $6.2: 9$ & $12: 43: 10.05$ & $+02: 36: 25.4$ & 6.99 & 133.9 & 18.55 & 1.24 & $-59 \pm 16$ & $-51 \pm 21$ & f01-27 & 0 \\
\hline $6.2: 26$ & $12: 43: 09.69$ & $+02: 37: 24.8$ & 6.27 & 127.9 & 21.81 & 3.32 & $44 \pm 58$ & $44 \pm 58$ & & 1 \\
\hline $6.2: 27$ & $12: 43: 09.32$ & $+02: 37: 28.1$ & 6.17 & 128.0 & 21.88 & 2.04 & $-137 \pm 42$ & $-137 \pm 42$ & & 1 \\
\hline $6.2: 50$ & $12: 43: 12.60$ & $+02: 38: 45.8$ & 6.20 & 113.8 & 20.17 & 0.56 & $-130 \pm 45$ & $-80 \pm 93$ & $6.1: 42$ & 0 \\
\hline $6.2: 53$ & $12: 43: 12.72$ & $+02: 39: 07.7$ & 6.09 & 110.5 & 21.29 & 2.68 & $23 \pm 50$ & $23 \pm 50$ & & 1 \\
\hline $6.2: 66$ & $12: 43: 06.26$ & $+02: 40: 05.8$ & 4.26 & 106.0 & 21.43 & 1.66 & $-86 \pm 62$ & $-86 \pm 62$ & & 1 \\
\hline $6.2: 67$ & 12:43:06.04 & $+02: 40: 07.7$ & 4.20 & 105.7 & 18.54 & 2.41 & $48 \pm 81$ & $48 \pm 81$ & & 1 \\
\hline $6.2: 77$ & 12:43:07.65 & $+02: 40: 45.3$ & 4.47 & 96.6 & 19.13 & 2.95 & $-30 \pm 48$ & $-30 \pm 48$ & & 1 \\
\hline $6.2: 79$ & 12:43:08.09 & $+02: 40: 51.6$ & 4.57 & 95.1 & 19.82 & 0.79 & $-193 \pm 22$ & $-196 \pm 32$ & f02-36 & 0 \\
\hline $6.2: 84$ & $12: 43: 15.79$ & $+02: 41: 12.5$ & 6.47 & 90.5 & 20.84 & 1.40 & $35 \pm 32$ & $35 \pm 32$ & & 1 \\
\hline $6.2: 93$ & $12: 43: 18.55$ & $+02: 41: 48.1$ & 7.18 & 85.7 & 21.33 & 3.65 & $-107 \pm 46$ & $-107 \pm 46$ & & 1 \\
\hline $6.2: 102$ & $12: 43: 09.33$ & $+02: 42: 21.7$ & 4.98 & 77.3 & 19.24 & 1.12 & $89 \pm 22$ & $112 \pm 31$ & f12-46 & 0 \\
\hline 7.1:6 & $12: 43: 35.38$ & $+02: 28: 57.5$ & 16.75 & 137.3 & 21.44 & 3.45 & $-47 \pm 48$ & $-47 \pm 48$ & & 1 \\
\hline 7.1:7 & $12: 43: 40.85$ & $+02: 29: 02.7$ & 17.65 & 133.8 & 20.80 & 3.85 & $-29 \pm 48$ & $-29 \pm 48$ & & 1 \\
\hline 7.1:11 & $12: 43: 35.58$ & $+02: 29: 16.4$ & 16.56 & 136.4 & 18.65 & 0.56 & $-20 \pm 22$ & $-20 \pm 22$ & & 1 \\
\hline $7.1: 15$ & $12: 43: 26.31$ & $+02: 29: 31.8$ & 14.85 & 142.2 & 19.83 & 0.95 & $313 \pm 20$ & $313 \pm 20$ & & 1 \\
\hline $7.1: 19$ & $12: 43: 41.26$ & $+02: 29: 46.6$ & 17.23 & 131.8 & 21.91 & 4.10 & $-31 \pm 40$ & $-31 \pm 40$ & & 1 \\
\hline $7.1: 20$ & $12: 43: 35.45$ & $+02: 29: 49.1$ & 16.14 & 135.2 & 20.52 & 1.49 & $256 \pm 27$ & $256 \pm 27$ & & 1 \\
\hline 7.1:30 & $12: 43: 41.27$ & $+02: 30: 29.1$ & 16.76 & 130.0 & 20.42 & 1.96 & $33 \pm 34$ & $33 \pm 34$ & & 1 \\
\hline $7.1: 31$ & $12: 43: 29.12$ & $+02: 30: 33.4$ & 14.52 & 137.5 & 21.71 & 1.15 & $131 \pm 35$ & $131 \pm 35$ & & 1 \\
\hline $7.1: 33$ & $12: 43: 30.99$ & $+02: 30: 40.4$ & 14.75 & 135.9 & 21.60 & 3.39 & $-37 \pm 58$ & $-37 \pm 58$ & & 1 \\
\hline $7.1: 36$ & $12: 43: 35.87$ & $+02: 30: 49.5$ & 15.52 & 132.3 & 21.92 & 0.59 & $70 \pm 70$ & $70 \pm 70$ & & 1 \\
\hline 7.1:41 & $12: 43: 33.03$ & $+02: 31: 10.1$ & 14.77 & 133.1 & 21.03 & 0.80 & $260 \pm 67$ & $260 \pm 67$ & & 1 \\
\hline 7.1:43 & $12: 43: 39.10$ & $+02: 31: 20.7$ & 15.80 & 128.9 & 18.54 & 3.00 & $-20 \pm 51$ & $-20 \pm 51$ & & 1 \\
\hline $7.2: 5$ & $12: 43: 32.92$ & $+02: 29: 02.1$ & 16.28 & 138.7 & 22.21 & 1.20 & $76 \pm 199$ & $76 \pm 199$ & & 1 \\
\hline $7.2: 19$ & $12: 43: 30.54$ & $+02: 29: 46.9$ & 15.33 & 138.5 & 21.08 & 3.81 & $50 \pm 85$ & $50 \pm 85$ & & 1 \\
\hline $7.2: 31$ & $12: 43: 22.37$ & $+02: 30: 31.2$ & 13.47 & 142.9 & 22.21 & 2.09 & $-55 \pm 50$ & $-55 \pm 50$ & & 1 \\
\hline $7.2: 34$ & $12: 43: 30.28$ & $+02: 30: 40.2$ & 14.63 & 136.4 & 22.61 & 1.61 & $19 \pm 61$ & $19 \pm 61$ & & 1 \\
\hline $7.2: 44$ & $12: 43: 25.03$ & $+02: 31: 12.1$ & 13.36 & 138.9 & 22.16 & 1.31 & $23 \pm 42$ & $23 \pm 42$ & & 1 \\
\hline
\end{tabular}


A\&A 544, A115 (2012)

Table D.1. continued.

\begin{tabular}{|c|c|c|c|c|c|c|c|c|c|c|}
\hline ID & $\begin{array}{c}\text { RA } \\
(\mathrm{J} 2000)\end{array}$ & $\begin{array}{c}\text { Dec } \\
(\mathrm{J} 2000)\end{array}$ & $\begin{array}{c}R_{4636} \\
{[\operatorname{arcmin}]}\end{array}$ & $\begin{array}{c}\text { PA } \\
{[\mathrm{deg}]}\end{array}$ & $\begin{array}{c}m_{R} \\
{[\mathrm{mag}]}\end{array}$ & $\begin{array}{l}C-R \\
{[\mathrm{mag}]}\end{array}$ & $\begin{array}{c}v_{\text {spec }} \\
{\left[\mathrm{km} \mathrm{s}^{-1}\right]}\end{array}$ & $\begin{array}{c}v_{\text {final }} \\
{\left[\mathrm{km} \mathrm{s}^{-1}\right]}\end{array}$ & Cross-Identification(s) & $1 \mathrm{st}$ \\
\hline $7.2: 51$ & $12: 43: 26.70$ & $+02: 31: 51.7$ & 13.15 & 135.6 & 20.94 & 0.76 & $97 \pm 60$ & $97 \pm 60$ & & 1 \\
\hline $7.2: 56$ & $12: 43: 33.91$ & $+02: 32: 09.4$ & 14.28 & 129.6 & 19.76 & 3.08 & $-8 \pm 42$ & $-8 \pm 42$ & & 1 \\
\hline $7.2: 61$ & $12: 43: 34.25$ & $+02: 32: 31.1$ & 14.12 & 128.3 & 21.57 & 1.64 & $-81 \pm 33$ & $-81 \pm 33$ & & 1 \\
\hline $7.2: 62$ & $12: 43: 26.58$ & $+02: 32: 33.5$ & 12.64 & 133.5 & 20.00 & 2.94 & $-11 \pm 53$ & $-11 \pm 53$ & & 1 \\
\hline $7.2: 67$ & $12: 43: 33.99$ & $+02: 32: 48.7$ & 13.89 & 127.5 & 21.11 & 3.28 & $31 \pm 43$ & $31 \pm 43$ & & 1 \\
\hline $7.2: 70$ & $12: 43: 28.94$ & $+02: 32: 57.7$ & 12.81 & 130.4 & 18.39 & 0.00 & $2 \pm 26$ & $2 \pm 26$ & & 1 \\
\hline $7.2: 71$ & $12: 43: 32.00$ & $+02: 33: 03.0$ & 13.35 & 128.0 & 21.99 & 1.76 & $211 \pm 35$ & $211 \pm 35$ & & 1 \\
\hline $7.2: 81$ & $12: 43: 32.18$ & $+02: 33: 44.1$ & 12.98 & 125.5 & 18.67 & 2.89 & $-21 \pm 55$ & $-21 \pm 55$ & & 1 \\
\hline $7.2: 96$ & $12: 43: 24.95$ & $+02: 34: 33.8$ & 11.03 & 127.4 & 18.52 & 0.88 & $272 \pm 11$ & $272 \pm 11$ & & 1 \\
\hline $7.2: 110$ & $12: 43: 22.13$ & $+02: 35: 22.2$ & 9.98 & 126.2 & 22.11 & 2.65 & $127 \pm 70$ & $127 \pm 70$ & & 1 \\
\hline $7.2: 112$ & $12: 43: 21.07$ & $+02: 35: 27.4$ & 9.72 & 126.7 & 21.47 & 0.92 & $-38 \pm 22$ & $-38 \pm 22$ & & 1 \\
\hline
\end{tabular}

